\title{
Analysis and Design of a Rotary Travelling-Wave \\ Oscillator for Integration in a Microwave Phased-Array

\author{
Transmitter
}

By

Kimia Taghizadeh Ansari, B.Sc., M.A.Sc.

A thesis submitted to the Faculty of Graduate and Postdoctoral Affairs in partial fulfillment of the requirements for the degree of

Doctor of Philosophy

in

Electrical Engineering

Carleton University

Ottawa, Ontario, Canada

(C) 2015, Kimia Taghizadeh Ansari 


\section{Abstract}

The design methodology and phase noise analysis of a multiphase bipolar Ku-band Rotary Travelling-Wave Oscillator (RTWO) is presented. Lack of symmetry in an RTWO can result in oscillation in an unwanted mode rather than travelling-wave mode. In order to determine the oscillation mode and also measure the phase shift, we have proposed a frequency domain phase shift measurement technique, intended for an $18 \mathrm{GHz}$ RTWO, which is presented. Using a $0.25 \mu \mathrm{m}$ BiCMOS process from NXP Semiconductors, the fabricated RTW-VCO gives access to eight different phases in steps of 45 degrees and achieves a tuning range of $1.2 \mathrm{GHz}$ centred at $17.5 \mathrm{GHz}$.

A beam-forming technique that utilizes the RTWO for LO phase shifting is also presented. In order to configure a two-element-array transmitter to show the functionality of an RTWO for beam-forming, we have designed and characterized a high frequency distribution network, a switching bank, a power amplifier (PA) and also a patch antenna. All of these components, except the antennas, have been integrated on a single, $19 \mathrm{~mm}^{2}$, silicon die along with the RTWO.

The measurement of the full system is performed with the aid of two off-chip antennas fabricated on a high frequency printed circuit board. The combined system is fully functional and the measurement results are provided. This research demonstrates the relevance of the RTWO for integration in phased-array transmitter systems. 


\section{Acknowledgment}

I would like to thank my friends and colleagues who contributed to the completion of this work. First and foremost, I am deeply indebted to my supervisor, Professor Calvin Plett, whose patience, encouragement and support helped me in finishing this work. Calvin, thanks for giving me the opportunity to study in the field that I have always been interested in and for being a welcome source of advice during this time. I have learnt a lot from you but the best lesson is to always be patient and generous, just like you.

I would like to express my sincere thanks to my industrial advisor, Dr. Patrice Gamand from NXP Semiconductors, for his endless technical support, enthusiasm and inspiration. Patrice, thanks for trusting me and providing me the great opportunity of working on such a big project. I cannot thank you enough and I should simply say that without your continuous support I could not have finished my work and write my thesis at this time.

I am also grateful to other NXP people who were involved in the delivery of their design kit to Carleton University, especially Dr. Olivier Tesson for his enormous help and technical support during this time.

I would like to thank the office administrators, technical staff and my fellow graduate students in the Department of Electronics who have always been there to listen, help and make my life easier. I am grateful to the excessive help and guidelines of our amazing departmental administrator, Ms. Blazenka Power, and our wonderful graduate assistant, 
Ms. Anna Lee. My warm thanks are due to all my friends in the blue room and particularly to the ones who helped me to finish this project and made this research period enjoyable.

My special and profound gratitude to my best friend Tyler Ross because of his help, technical advice and for always being there to listen and bring a smile to my face. Tyler, thanks for everything; not only because of your technical advice, but also for the nights you stayed in the dark blue room along with me until very late and for the faith you always had in me and the end result of this research.

I wish to thank my earlier mentors especially Dr. Mustapha Yagoub from University of Ottawa. Without him and his generous support I could not have been in this position and pursuing my studies in my desired field.

My most sincere thanks are reserved to my husband, Reza, for bearing with me throughout this adventure from the beginning all the way to organizing my after-defence party. Reza, without your unconditional support, love and encouragement, I could have never finished this work.

Last but not least, I owe my very deep and loving thanks to my family, my father, my mother, my two sisters and my brother whose support and love enabled me to pursue my program far from home. I never get used to living far from you and you are always in my heart. 


\section{Table of Contents}

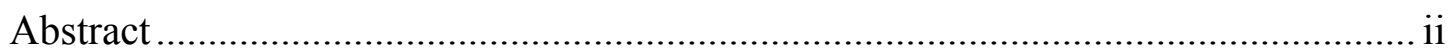

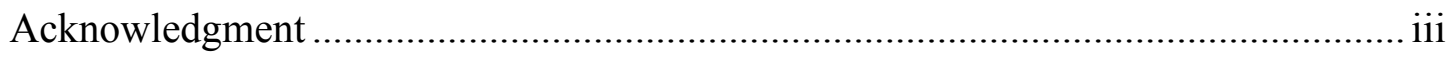

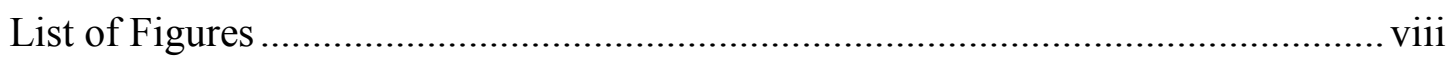

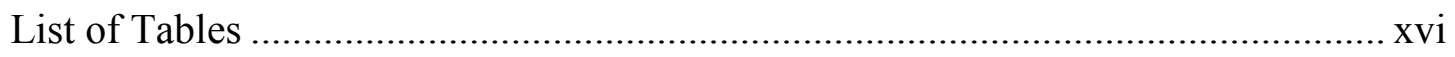

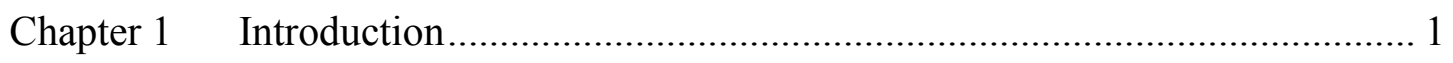

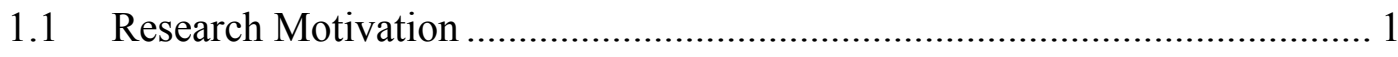

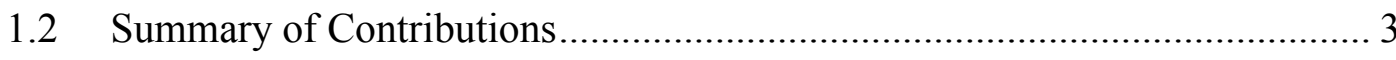

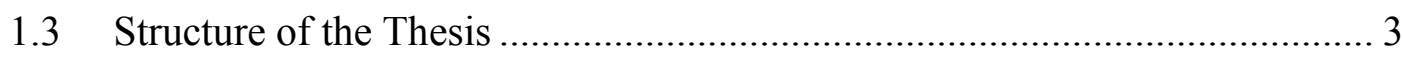

Chapter 2 Overview of the State of the Art ....................................................... 5

2.1 Introduction of Integrated RF Oscillators ................................................... 5

2.2 Transmission-line-based Voltage Controlled Oscillators .............................. 10

2.2.1 Standing Wave Oscillators (SWO) ............................................................ 11

2.2.2 Travelling-wave Oscillators (TWO) .............................................................. 17

2.2.3 Rotary Travelling-Wave Oscillator (RTWO) …………………………….....2

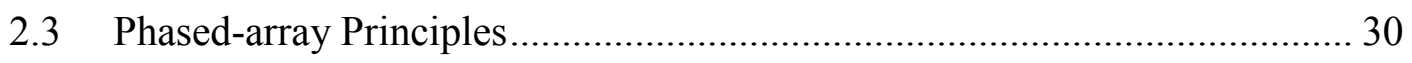

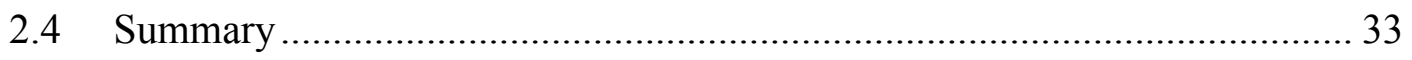

Chapter 3 Design of a Ku-band Rotary Travelling-wave Oscillator ...................... 36 


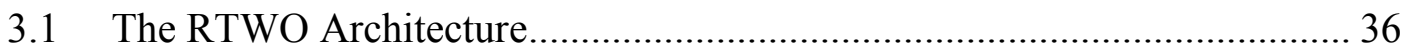

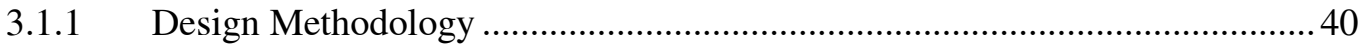

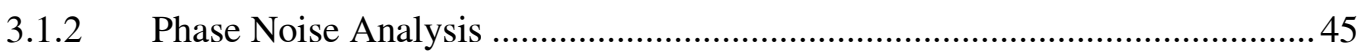

3.2 Post-Layout Simulation Results........................................................... 56

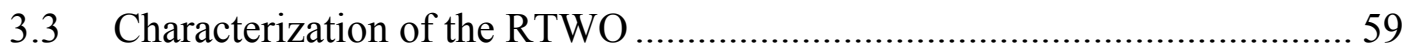

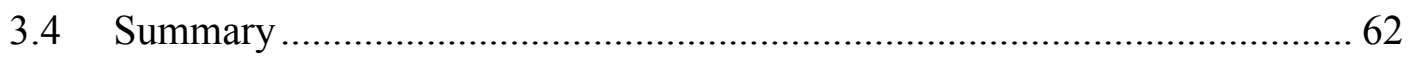

Chapter 4 RTWO Phase Shift Measurement .................................................. 65

4.1 Conventional Phase Shift Measurement Approaches ................................ 65

4.2 Innovative Frequency Domain Phase Shift Measurement Technique ......... 68

4.2.1 Calibration of the Measurement Setup ...................................................... 72

4.2.2 Voltage Calculation and Power Simulation of the Complete Lossy Setup ..... 74

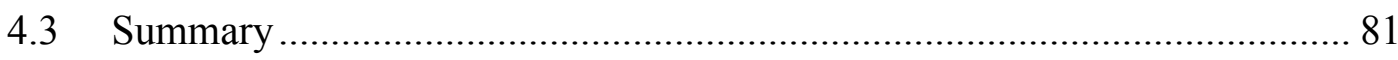

Chapter 5 Ku-band Integrated Building Blocks for a Phased-Array Transmitter

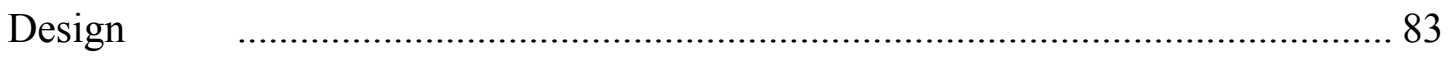

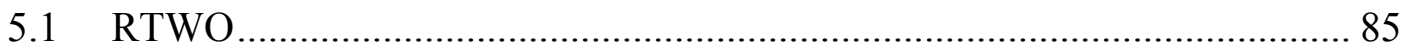

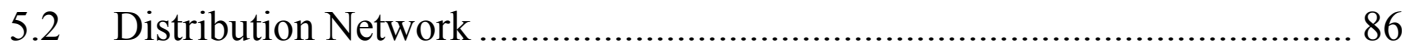

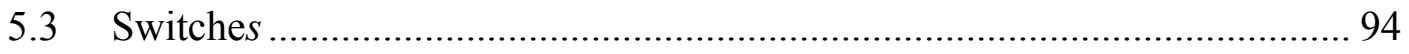

5.3.1 Isolating the Substrate Contact of the Switch.............................................. 98

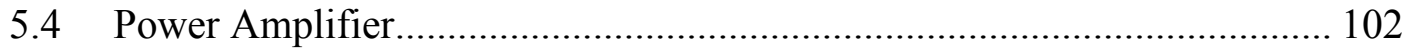


5.4.1 EM Simulation Results of the Power Amplifier......

5.4.2 Characterization of the PA

5.5 Coplanar Patch Antenna ..................................................................... 113

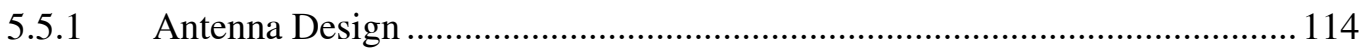

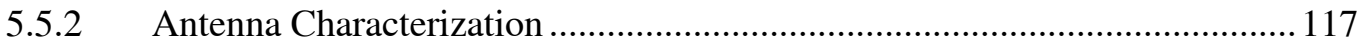

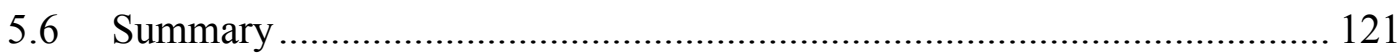

Chapter 6 Fully Integrated Two-Element Array Transmitter Utilizing an RTWO ...

125

6.1 Monolithic Two-Element Array Transmitter .......................................... 128

6.2 Printed Circuit Board with Antennas .................................................... 135

6.3 Measurement Results of the Full System.............................................. 136

6.3.1 Phase Shift Measurement Using Power Combiner....................................... 141

6.3.2 Beam-Forming Measurement Using Physical Antennas .............................. 150

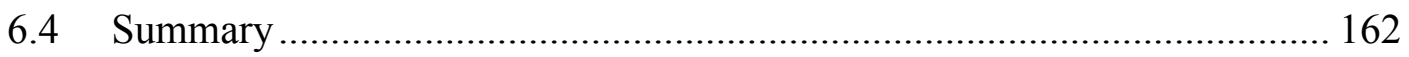

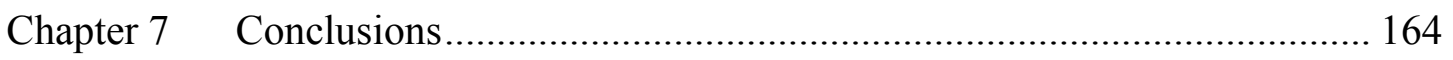

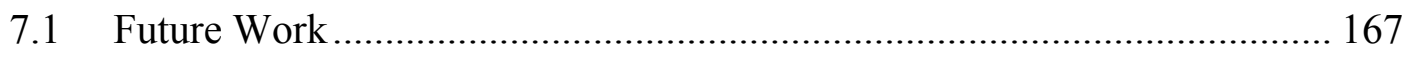

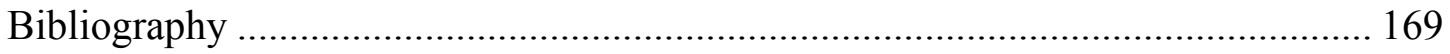




\section{List of Figures}

Figure 2.1 Output spectrum of a real oscillator showing the effect of phase noise- 6

Figure 2.2 Linear model for an oscillator---_---- 7

Figure 2.3 Example of different topologies generating negative resistance --------- 8

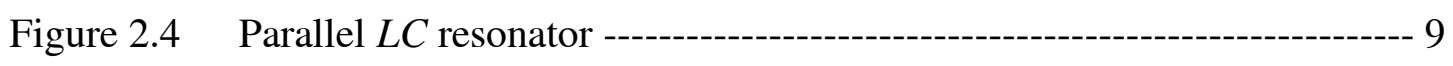

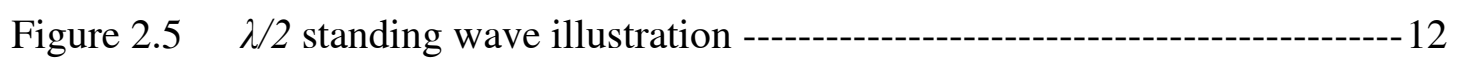

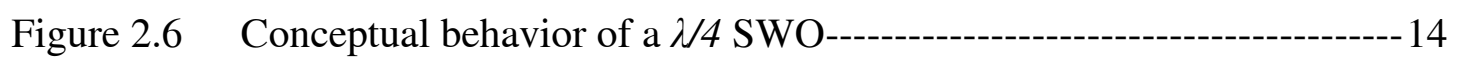

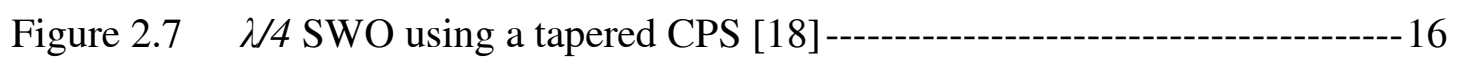

Figure 2.8 Four-stage resistively terminated distributed amplifier-------------------18

Figure 2.9 Distributed oscillator realized from a distributed amplifier--------------19

Figure 2.10 RTWO formed from a distributed amplifier --------------------------24

Figure 2.11 Symmetric resonator geometry with one cross-over [31] ------------26

Figure 2.12 Phased-array architectures (a) IF phase shift (b) RF phase shift (c)

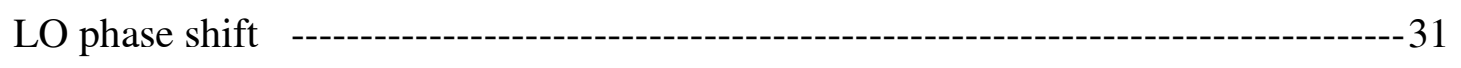

Figure 2.13 Conceptual $n$-element phased-array transmitter using an RTWO ---32

Figure 3.1 Cross-coupled NPN transistors forming a $-g_{m}$ topology -----------------37 
Figure 3.2 Characterization of the differential NMOS varactors at $18 \mathrm{GHz}$ $-38$

Figure 3.3 Schematic diagram of the proposed RTW-VCO

Figure 3.4 The RF model of one segment of the RTW-VCO $-41$

Figure 3.5 Equivalent circuit of our RTW-VCO used to calculate the phase noise48

Figure 3.6 Differential pair input voltage and output current showing the noise model $-50$

Figure 3.7 Switching pair $I-V$ curve and transconductance of the switching pair -51

Figure 3.8 Simulated output spectrum of the RTWO showing the fundamental tone 57

Figure 3.9 Simulated phase noise at a $1 \mathrm{MHz}$ offset from the carrier frequency --58

Figure 3.10 Simulated transient response of the RTWO $-59$

Figure 3.11 Die photomicrograph of the RTWO fabricated in NXP $0.25 \mu \mathrm{m}$

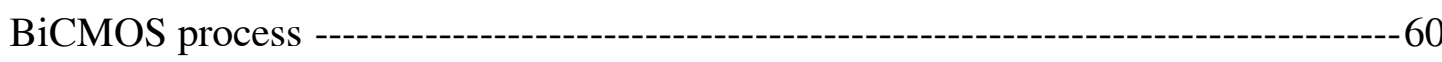

Figure 3.12 Measured output spectrum of the RTWO showing the fundamental at $17 \mathrm{GHz}$ $-61$

Figure 3.13 Measured phase noise at a $1 \mathrm{MHz}$ offset from the $17 \mathrm{GHz}$ centre frequency $-61$

Figure 4.1 DC component of a diode-based mixer's IF output $-67$ 
Figure 4.2 The RTWO schematic

Figure 4.3 Simulated output spectrum under standing wave mode condition ------70

Figure 4.4 Simulated time domain voltage under standing wave mode condition-70

Figure 4.5 Measurement setup used to test our innovative approach-----------------71

Figure 4.6 The output power of the RTWO at $A_{l}$ and $A_{3}$ nodes ---------------------- 74

Figure 4.7 Schematic diagram of Figure 4.5 for voltage calculation ----------------- 75

Figure 4.8 Applying superposition and cancelling the effect of the right segment's output $-75$

Figure 4.9 Summation of two vectors with $\Delta \boldsymbol{\varphi}^{\circ}$ of phase shift between them ----- 77

Figure 4.10 Schematic diagram to simulate the output power of the lossy setup 78

Figure 4.11 Simulated combined output power for different phase shifts---------79

Figure 4.12 Measured combined output powers from $A_{1}$ and $A_{3}$--------------------80

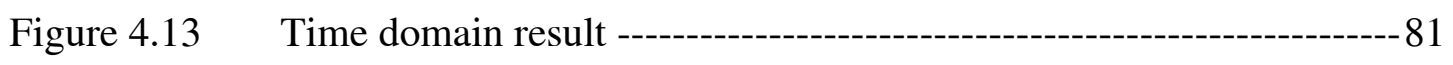

Figure 5.1 The 4-element array transmitter utilizing an RTWO----------------------84

Figure 5.2 Typical H-tree distribution network -----------------------------------------87

Figure 5.3 Meandered H-tree network ---------------------------------------------------88

Figure 5.4 HFSS simulation results of the meandered H-tree network--------------89 
Figure 5.5 Our designed distribution network for four-element array----------------90

Figure 5.6 EM simulation results of our distribution network-------------------------90

Figure 5.7 EM simulation results of the distribution network for two-element array signal distribution---------------------------------------------------------------------------------93

Figure 5.8 Circuit schematic of a series transistor in ON state -----------------------95

Figure 5.9 Circuit schematic of a shunt transistor in OFF state -----------------------96

Figure 5.10 Schematic diagram of the proposed series/shunt/series switch with

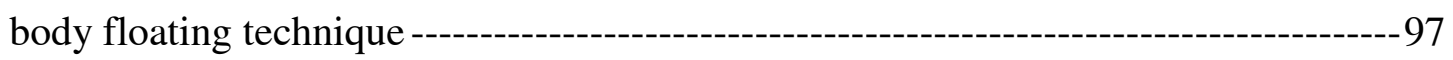

Figure 5.11 Layout of the proposed series/shunt/series switch ---------------------99

Figure 5.12 Performance summary of the switch in ON state--------------------- 100

Figure 5.13 Performance summary of the switch in OFF state ------------------- 101

Figure 5.14 Schematic diagram of our three-stage class AB power amplifier-- 103

Figure 5.15 Collector current of the last stage-------------------------------------- 104

Figure 5.16 Full EM simulation results of the PA --------------------------------- 106

Figure 5.17 EM simulated results of the PA showing $1 \mathrm{~dB}$ compression point and saturated power ----------------------------------------------------------------------------------- 107

Figure 5.18 EM simulated PAE of the PA -------------------------------------------- 108 
Figure 5.19 EM simulated stability factor of the amplifier showing the amplifier is unconditionally stable 109

Figure 5.20 Die photomicrograph of the PA----------------------------------------- 110

Figure 5.21 Measured small-signal parameters of the PA -------------------------- 110

Figure 5.22 Measured power performance of the PA ------------------------------- 111

Figure 5.23 Calculated stability factor obtained from measured S-parameters- 112

Figure 5.24 Coplanar antenna structure used in our design ----------------------- 115

Figure 5.25 HFSS simulation results of the antenna showing the $-10 \mathrm{~dB}$ bandwidth ------------------------------------------------------------------------------- 116

Figure 5.26 HFSS simulation results for the antenna radiation pattern ---------- 117

Figure 5.27 Photograph of the antenna and two antennas with an $8 \mathrm{~mm}$ separation 118

Figure 5.28 Measured $S_{11}$ results for the antenna shown in Figure 5.24 for three different distances between the antenna and the probe chuck. Distance 1 is equal to the thickness of a sheet of paper, Distance 2 is equal to $2.5 \mathrm{~mm}$, and Distance 3 is equal to $5 \mathrm{~mm}$ 120

Figure 5.29 Measured $\mathrm{S}_{12}$ results for the two antennas for two different distances between the antenna and the probe chuck. Distance 1 is equal to $2.5 \mathrm{~mm}$ and Distance 2 is equal to $5 \mathrm{~mm}$ 120 
Figure 6.1 Schematic diagram of the full system

Figure 6.2 Die photo-micrograph of the two-element array transmitter 130

Figure 6.3 EM simulation results of the full system for RTWO's $45^{\circ}$ signals --- 132

Figure 6.4 EM simulation results of the full system for the RTWO's $90^{\circ}$ signals----

Figure 6.5 EM simulation results of the full system for the RTWO's $135^{\circ}$ signals --

Figure 6.6 Photograph of the printed circuit board 135

Figure 6.7 Photo of the printed circuit board and measurement setup. (a) printed circuit board (b) measurement setup 137

Figure 6.8 Output power of the top element while the bottom element is terminated to $50 \Omega$ 138

Figure 6.9 Output power of the bottom element while the top element is terminated to $50 \Omega$

Figure 6.10 Output power of the top element for $V_{\text {tune }}=2.5 \mathrm{~V}$ 140

Figure 6.11 Measured output power of the top element while all switches are OFF 
Figure 6.12 Measured combined output power of the transmitter after being switched for $90^{\circ}$ of phase shift 144

Figure 6.13 Measurement setup used to measure the phase shift using a power combiner 145

Figure 6.14 Simulated combined output power used to calculate $90^{\circ}$ of phase shift 146

Figure 6.15 Output of the bottom element while the $A_{2}$ switch is $\mathrm{ON}$ 147

Figure 6.16 Combined output power of the top and bottom elements after being switched for $135^{\circ}$ of phase shift

Figure 6.17 Comparison of theoretical (red), EM-simulated (purple) and measurement results (green) of phase shift using a power combiner for phase shift measurement

Figure 6.18 HFSS geometry for two antennas being $8 \mathrm{~mm}$ apart152

Figure 6.19 HFSS simulation results for the radiation pattern of two antennas beside each other fed by signals with different phase shifts 153

Figure 6.20 Measurement setup used to measure the output power and amplitude mismatch 155

Figure 6.21 Radiation pattern of the antenna after being extended and tapered on the PCB 157 
Figure 6.22 Measured radiation pattern for radiated angle of $0^{\circ}$

Figure 6.23 Measured radiation pattern for radiated angle of $\pm 15.78^{\circ}$

Figure 6.24 Measured radiation pattern for radiated angle of $\pm 32.95^{\circ}$---------- 160

Figure 6.25 Measured radiation pattern for radiated angle of 54.65 ----------- 160

Figure 6.26 Radiation pattern of the antennas showing the beam-forming capability of the two-element array transmitter ------------------------------------- 161 


\section{List of Tables}

Table 1 Comparison of SWOs, TWOs and RTWOs

Table 2 Main characteristics of Qubic4XI $0.25 \mu \mathrm{m}$ BiCMOS process from NXP

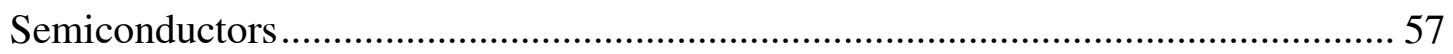

Table 3 Performance Summary of our RTWO compare to other recently published

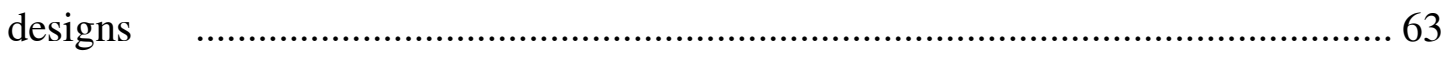

Table 4 Performance summary of the PA........................................................ 113

Table 5 Rogers RO4003 material characteristics................................................ 114

Table 6 Antenna dimensions based on Figure 5.24 ......................................... 116

Table 7 Summary of the calculated radiated angles from the RTWO's phase shift..

150 


\section{List of Abbreviations and Symbols}

BiCMOS Bipolar complementary metal oxide semiconductor

CMOS Complementary metal oxide semiconductor

$C_{c B} \quad$ Collector-to-bulk capacitance of a BJT

$C_{\mu} \quad$ Base-to-collector capacitance of a BJT

$C \pi \quad$ Base-to-emitter capacitance of a BJT

dB Decibels

$\mathrm{dBm} \quad 10 \log ($ Power in Watts/1 mW)

$\mathcal{E}_{\mathrm{r}} \quad$ Dielectric permittivity

EIRP Effective isotropic radiated power

$f_{T} \quad$ Unity current-gain frequency

$f_{\max } \quad$ Maximum frequency of oscillation

GaAs Gallium Arsenide

$G_{m} \quad$ Large signal transconductance

$g_{m} \quad$ Small signal transconductance

HB Harmonic balance simulation 


\begin{tabular}{ll} 
IF & Intermediate frequency \\
InP & Indium phosphide \\
Ku-band & 12 GHz-18 GHz band of frequency \\
KVCO & VCO gain \\
$k$ T & Product of Boltzman constant and the temperature equal to $4.11 \times 10^{-21} \mathrm{~J}$ \\
$\lambda$ & Wavelength \\
LO & Local oscillator \\
$\mu_{0}$ & Free space permeability equal to $4 \pi \times 10^{-7} \mathrm{H} / \mathrm{m}$ \\
PA & Power amplifier \\
TWO & Travelling wave oscillator \\
RTWO & Rotary travelling wave oscillator \\
PN & Phase noise of an oscillator \\
PSS & Periodic steady-state simulation \\
& \\
\hline
\end{tabular}


VCO Voltage controlled oscillator

$v_{\text {phase }} \quad$ Phase velocity in a transmission line

$v_{T} \quad$ Thermal voltage

$\mathrm{Z}_{0} \quad$ Characteristic impedance

xix 


\section{Chapter 1 Introduction}

\subsection{Research Motivation}

There has been a widespread and growing demand for ultra-high-speed communication systems in recent years. This ever increasing demand has driven the telecommunications industry to explore techniques for designing on-chip high-frequency oscillators in cost-efficient technologies.

Systems operating at gigahertz rates require generators with low skew and low phase noise. In the past, such high-frequency circuits have been implemented in compound semiconductor technologies such as GaAs or InP, although CMOS and BiCMOS technologies are now regarded as the most promising technologies for designing RF and microwave circuits due to their large scale integration capabilities.

However, silicon-based technologies suffer from parasitic components both in active and passive devices. This makes it necessary to pursue alternative approaches for the design of high-speed circuits, such as distributed techniques.

Distributed oscillators originate from distributed amplifiers and they have been widely used for wide-band applications. A distributed amplifier achieves a higher gainbandwidth product by absorbing the parasitic components into transmission lines and essentially trades higher delay for a better gain-bandwidth product. Inherently, a 
distributed oscillator can operate at frequencies close to the cut-off frequency of the loaded resonator.

Among different transmission-line-based oscillators, rotary travelling-wave oscillators (RTWOs) have the unique property of sustaining a travelling-wave while achieving high output power and low phase noise with low power consumption. The low power consumption of this structure is due to the fact that energy is recycled by the basic operation of the RTWO [1].

The two main applications for an RTWO are to be used in a high frequency clock distribution network for creating $I / Q$ signals [2], [3] or multiphase phased-array transceivers [4], [5].

Transmitter beam-forming and receiver spatial selectivity approaches have been adopted in the form of integrated phased-array systems to overcome the path-loss, attenuation and interference. In phased-array receivers, the noise figure and signal-tonoise ratio (SNR) can be improved by implementing multiple antennas to receive the transmitted signal [6]. Meanwhile, one of the main challenges of integrated silicon-based transmitters is the design of high output power, high efficiency power amplifiers. Phasedarray systems are regaining interest because of future requirements for high performance and energy efficient radio links.

In order to design an integrated phased-array transceiver, a component which can create uniform phase shift either in the Intermediate Frequency (IF) [7], Radio Frequency (RF) [8] or Local Oscillator (LO) [9] path is required. However, among different methods 
of phase shifting to design a phased-array transmitter, the LO phase shifting eliminates the need for variable gain amplifiers in the system as long as the LO signal power is high enough to fully switch the mixer's switching transistors.

In this specific research work, the design and characterization of a Ku-band RTWO is presented. The fabricated circuit is then used in a phased-array transmitter design, incorporating a distribution network, switching banks and power amplifiers, all on a custom-designed BiCMOS integrated circuit, as well as off-chip antennas on a high frequency printed circuit board. The objective is to use these components in an LO phase shifting frequency system, in order to do beam-forming at Ku-band of frequency and to show the proof of the concept.

\subsection{Summary of Contributions}

Along with the design of the individual components comprising the phased-array transmitter, the three principal contributions proposed are:

- a complete phase noise analysis of a bipolar RTWO;

- an innovative, high frequency, low cost method for phase shift measurements; and

- the monolithic integration of the multi-phase RTWO into a full phased-array system and its validation.

\subsection{Structure of the Thesis}

This remainder of this thesis is structured as follows: 
- Chapter 2 reviews the benefits of phased-array systems and the different types of high-frequency transmission-line-based oscillators that can be used in them.

- Chapter 3 presents a detailed study on the design and characterization of a fully integrated eight-phase Ku-band rotary travelling-wave voltage-controlled oscillator (RTW-VCO). The analytical calculations and step-by-step analysis of the phase noise of a bipolar RTWO is also provided in this chapter.

- Chapter 4 describes a nontraditional, low barrier-to-entry, frequency domain approach for phase measurement of a multiphase, Ku-band RTWO.

- Chapter 5 explains the design methodology and layout considerations of different building blocks needed for the design of a Ku-band integrated phased-array transmitter employing an RTWO. This chapter shows the design and characterization of integrated Ku-band distribution network, bank of switches, power amplifier and off-chip patch antenna.

- Chapter 6 shows the assembly of the silicon building blocks explained in Chapter 5 , as a monolithic system, and the challenges of connecting it to off-chip antennas on a printed circuit board. The measurement results of the full system are presented in this chapter.

- Lastly, Chapter 7 presents our conclusions and discusses future work. 


\section{Chapter 2 Overview of the State of}

\section{the Art}

This chapter provides a short overview of different transmission-line-based oscillators and their potential advantages and disadvantages. It also includes a brief discussion of previously published transmission-line-based oscillator topologies and phase noise, in order to make a fair comparison between them at the end of the chapter. A short discussion of the general principles of phased-array transmitters, as a potential application for multiphase wave-based oscillators is also provided.

\subsection{Introduction of Integrated RF Oscillators}

An electrical oscillator is a circuit generating periodically time varying waveforms using only DC supplies. In most RF applications, clean sinusoidal reference is required to down-convert the received signals to be used in the base-band or up-convert the modulated signals to the RF frequency.

One of the most important characteristics of an oscillator is its output power and phase noise. In other words, we would like the output spectrum of an oscillator to be a strong tone only at the desired frequency or at multiples of the oscillation frequency. This is because noise spectrum near the carrier frequency will also be converted either into the 
base-band part of a receiver or into the RF frequency path of a transmitter and this will degrade the SNR of the radio link.

However, all physical oscillators have a skirt shape power spectral density as shown in Figure 2.1. This means that the generated power is not concentrated at the fundamental tone and it leaks into the neighboring frequencies. This skirt shape of the oscillator's output spectrum is evidence of the phase noise.

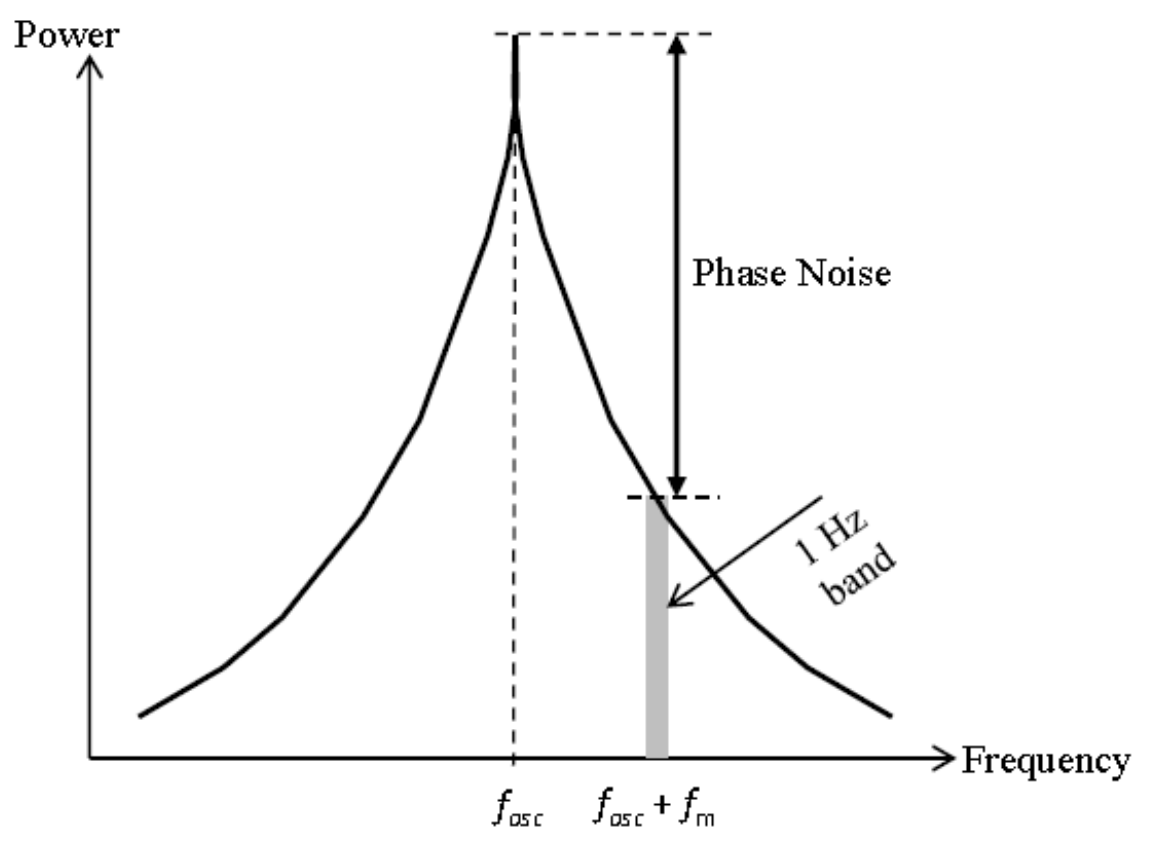

Figure 2.1 Output spectrum of a real oscillator showing the effect of phase noise

Figure 2.2 and equations (2.1) - (2.2), show a linear oscillator model and the Barkhausen criteria for oscillation start-up. The criteria states that to sustain an oscillation with constant amplitude, the open loop gain of the system should be greater than one and the phase around the loop should be a multiple of $2 \pi[\mathbf{1 0}]$. 


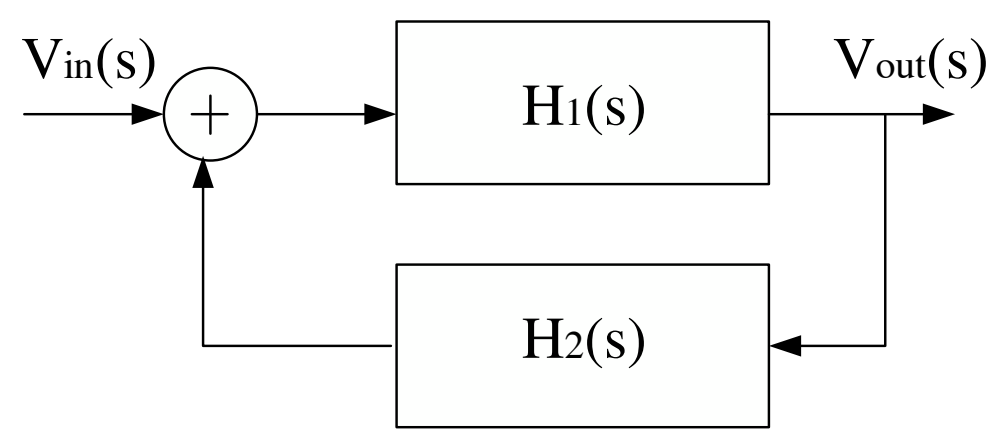

Figure 2.2 Linear model for an oscillator

$$
\begin{gathered}
\left|H_{1}(j \omega)\right|\left|H_{2}(j \omega)\right| \geq 1 \\
\measuredangle H_{1}(j \omega) H_{2}(j \omega)=2 n \pi \\
n \in 0,1,2 \ldots .
\end{gathered}
$$

where $H_{1}$ and $H_{2}$ are the transfer functions of all blocks around the loop and the product of them is the open-loop gain.

In order to meet the Barkhausen criteria and the steady state condition, a positive feedback which can generate negative resistance is required to sustain the oscillation and overcome the loss of the resonator [11].

There are different topologies, shown in Figure 2.3, which can generate negative resistance and they have been widely used in different applications [12] - [13]. Figure 2.3(b) shows the most commonly used topology, the so-called $-g_{m}$ topology, for an oscillator design. This topology is practical because of its simplicity and also because it is inherently differential. Furthermore, in the $-g_{m}$ topology the minimum required transconductance of the transistors to start up the oscillation, only depends on the 
resonator's loss and not on the oscillation frequency or the transistor's parasitic components.
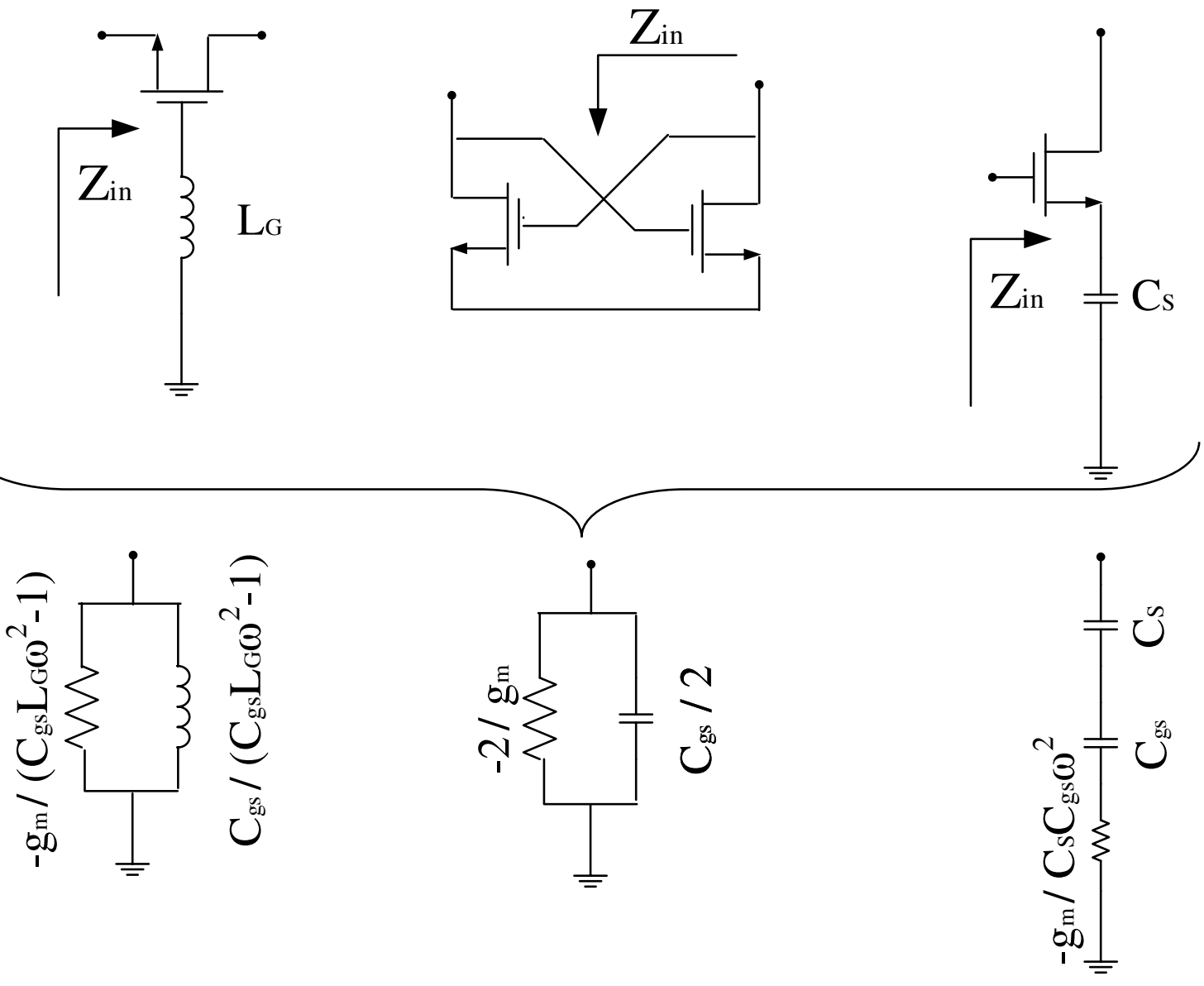

(a)

(b)

Figure 2.3 Example of different topologies generating negative resistance

The most commonly used integrated RF oscillators are based on a parallel $L C$-tank resonator, as shown in Figure 2.4. In these oscillators, the $L C$ resonator determines the oscillation frequency after reaching the steady-state condition. 
The oscillation angular frequency and quality factor of $L C$-tank oscillators are determined based on (2.3) and (2.4), respectively.

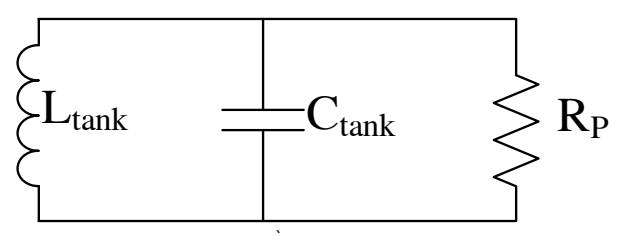

\section{Figure 2.4 Parallel $L C$ resonator}

$$
\begin{gathered}
\omega_{\text {osc }}=\sqrt{\frac{1}{L_{\text {tank }} C_{\text {tank }}}} \\
Q=\frac{R_{p}}{\omega_{0} L_{\text {tank }}}
\end{gathered}
$$

where $L_{\text {tank }}$ is the total inductance of the resonator, $C_{\text {tank }}$ is the total capacitance of the oscillator including the parasitic capacitances of the transistors, and $R_{p}$ is the parallel resistance of the resonator.

However, as the frequency goes higher, it becomes increasingly difficult to achieve the desired specifications, using $L C$-tank resonators. Operating at multi-gigahertz frequencies, the $L C$ product should decrease dramatically while normally the inductor loss and parasitic capacitance of the transistors do not scale down at the same rate. This effect results in the choice of an even smaller inductor to oscillate at the defined frequency.

Using a very small inductor to design a high frequency oscillator corresponds to high power dissipation. This is due to the fact that as the inductance gets smaller, its quality 
factor does not scale up proportionately, and thus the output swing is reduced for the same value of current. This means that for a constant output power, higher current consumption is unavoidable. Furthermore, a very small inductance makes the circuit very sensitive to the parasitic inductance of interconnects, and evaluating the value of the inductance to predict the oscillation frequency will be very difficult.

Researchers have therefore looked to alternative oscillator topologies for better clock performance at high frequencies. Among different techniques, wave-based oscillators have been comprehensively studied.

In contrast to $L C$-tank oscillators, the operation of the wave-based oscillators depends on the behavior of the wave propagation on a transmission line and these oscillators can effectively operate up to the cut-off frequency of their loaded resonator.

\subsection{Transmission-line-based Voltage Controlled Oscillators}

Distributed oscillators originate from distributed amplifiers and they have been widely used for wide-band RF and microwave applications. Distributed amplifiers or travelling-wave amplifiers have a more relaxed gain-bandwidth tradeoff compared to traditional amplifiers since the transistor parasitic capacitances are absorbed into the transmission lines [14]. Inherently, a distributed oscillator can operate at frequencies close to the $f_{\max }$ of the transistor.

For moderate-frequency VCO implementations, the $L C$-tank is widely used because of its superior phase noise performance, small size and simplicity. However, at higher 
frequencies the relatively high power consumption of an $L C$-tank oscillator, compare to wave-based oscillators, makes it an imperfect candidate for an oscillator design.

Alternatively, since the operation of wave-based oscillators is depending on the propagation of the wave in a transmission line, they can operate up to the cut-off frequency of the resonator with much less power consumption. These circuits can be categorized into three main groups, namely, Standing-Wave Oscillators (SWO), Travelling-Wave Oscillators (TWO) and Rotary Travelling-Wave Oscillators (RTWO).

\subsubsection{Standing Wave Oscillators (SWO)}

A standing wave in general is formed when two identical waves that are propagating in opposite directions, interact. A simple way to generate a standing wave is to send an incident wave to a transmission line and reflect it back with a lossless termination such as a short circuit [15].

Figure 2.5 shows half-wavelength SWO, where a cell of cross-coupled inverters injects a signal in the middle of the $\lambda / 2$ resonator. The signal is injected in the middle and the wave starts to travel in both the left and right directions. Using an ideal electrical short at the end of the resonator, the travelling-waves are completely reflected with an opposite phase. They add up to the injected signal and show maximum voltage amplitude in the middle of the $\lambda / 2$ resonator $[\mathbf{1 6}]$.

The general case of two waves travelling in opposite directions with different phase and amplitudes can be described based on (2.5) and (2.6), respectively. 


$$
\begin{gathered}
V_{a}(t, x)=A_{0} \cos \left(\omega_{0} t-\beta x\right) \\
V_{b}(t, x)=B_{0} \cos \left(\omega_{0} t+\beta x+\varphi\right)
\end{gathered}
$$

A standing wave is formed if these two waves interact, based on (2.7):

$$
\begin{gathered}
V_{a}(t, x)+V_{b}(t, x)= \\
2 B_{0} \cos \left(\beta x+\frac{\varphi}{2}\right) \cos \left(\omega_{0} t+\frac{\varphi}{2}\right)+\left(A_{0}-B_{0}\right) \cos \left(\omega_{0} t-\beta x\right)
\end{gathered}
$$

The first term in (2.7) shows the standing wave with a constant phase and different amplitude based on the position, $x$, across the resonator, and the second term shows the travelling-wave signal with a constant amplitude but different phase based on the position on the resonator.

However, if the circuit is perfectly symmetrical then $A_{0}=B_{0}$, and the second term cancels and only the standing wave term remains. It can also be stated from (2.7) that the voltage amplitude of the standing wave term is twice of that in the travelling-wave term [17].

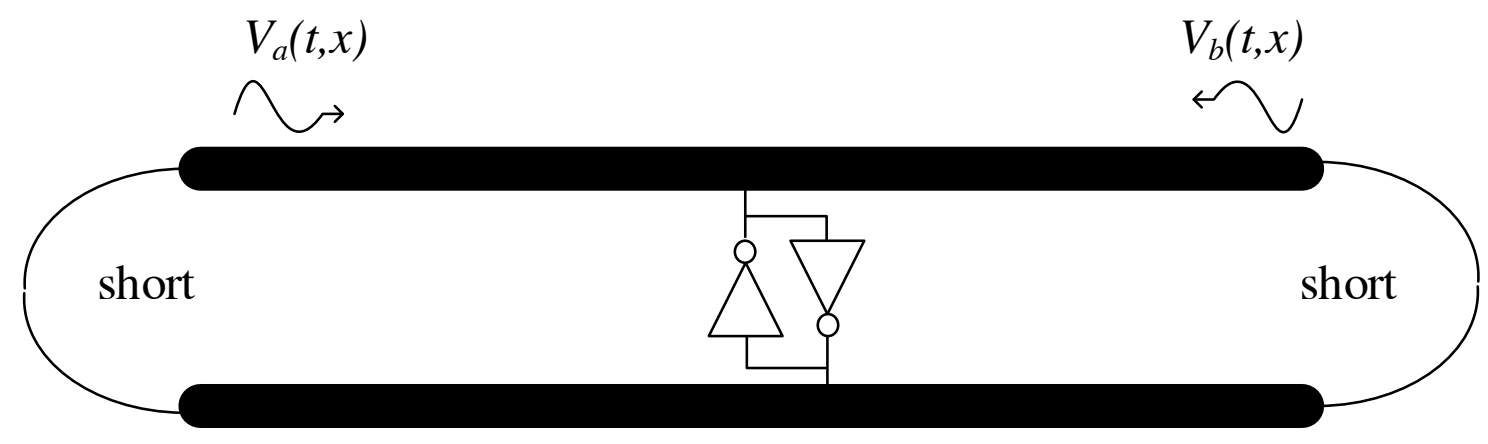

Figure 2.5 $\quad \lambda / 2$ standing wave illustration 
The skew and jitter of distributed signals in a clock distribution network utilizing SWOs, can be significantly reduced. This is due to the fact that in SWOs the output phases are not depending on the position across the ring and ideally, this leads to no skew between the output signals.

SWO design has been well studied in the literature. In [18], the authors have taken advantage of the amplitude variation of an SWO to taper the transmission line and lower the phase noise of the oscillator.

Figure 2.6 shows the conceptual voltage and current waves of a $\lambda / 4$ SWO which has been modified in [18] in order to improve the phase noise of the oscillator.

According to this figure, at the short end, $(z=l)$, there is a voltage minimum and a current maximum. At the other end, $(z=0)$, where the cross-coupled inverters are located, there is a voltage maximum and a current minimum.

The differential transmission line of Figure 2.6 can be implemented with a coplanar stripline (CPS) structure which can be modeled with familiar differential $R L C G$ lumped components, where $R, L, C$ and $G$ are series resistance and inductance, and shunt capacitance and conductance per-unit-length of the CPS, respectively.

In general, series resistance couples to the current waves and shunt conductance couples to the voltage waves and they introduce series and shunt losses which lower the quality factor, $Q$, of the line and consequently the phase noise. 

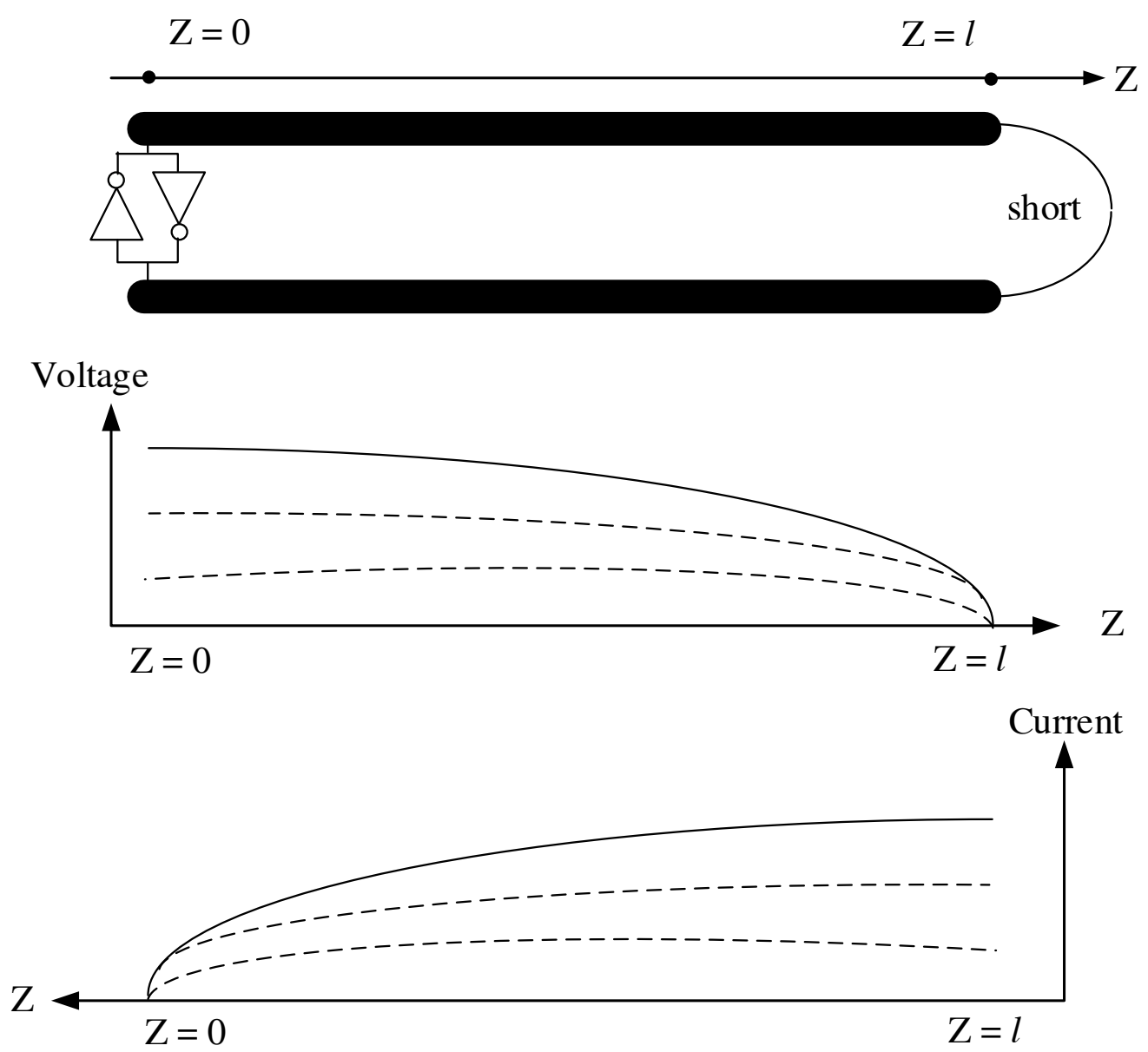

Figure 2.6 Conceptual behavior of a $\lambda / 4$ SWO

The phase noise contribution of the tank of a $\lambda / 4$ SWO utilizing a $-g_{m}$ gain cell, at an offset frequency $\omega_{\mathrm{m}}$, can be evaluated based on (2.8) [19]:

$$
\begin{gathered}
\mathcal{L}\left(\omega_{m)_{\text {tank-STWO }}}=\frac{4 k T R_{p}}{V_{0}^{2}}\left(\frac{\omega_{0}}{2 \omega_{m} Q}\right)^{2}\right. \\
Q=\frac{\pi^{2} R_{P}}{8 L \omega_{0}} \\
V_{0}=\frac{4 I_{\text {tail }} R_{p}}{\pi}
\end{gathered}
$$


where $R_{p}$ is the parallel resistance of the resonator, $V_{0}$ is the oscillator's output voltage, $I_{\text {tail }}$ is the total tail DC current and $Q$ is the quality factor of the resonator.

It should be noted that the quality factor of a distributed resonator is not the same as for an $L C$-tank. The value of $Q$ in (2.8) has been calculated based on the input impedance of the resonator at an offset frequency of $\omega_{\mathrm{m}}$, and the 3-dB bandwidth definition shown in (2.9) and (2.10), respectively.

$$
\begin{gathered}
Z_{\text {in }}\left(\omega_{0}+\omega_{m}\right)=Z_{0}\left(\frac{Z_{L}+j Z_{0} \tan \beta l}{Z_{0}+j Z_{L} \tan \beta l}\right) \\
Z_{\text {in }}\left(\omega_{0}+\omega_{3-d B}\right)|| R_{p} \rightarrow\left|Z_{e q}\left(\omega_{0}+\omega_{3-d B}\right)\right| \\
=\left|\frac{R_{p} Z_{i n}\left(\omega_{0} \pm \omega_{3-d B}\right)}{R_{p}+Z_{\text {in }}\left(\omega_{0} \pm \omega_{3-d B}\right)}\right|=\frac{R_{p}}{\sqrt{2}} \rightarrow \\
R_{p}=\left|Z_{\text {in }}\left(\omega_{0}+\omega_{3-d B}\right)\right| \rightarrow Q=\frac{\omega_{0}}{2 \omega_{3-d B}}
\end{gathered}
$$

where $Z_{0}$ is the characteristic impedance of the resonator, $Z_{L}$ is the load impedance and $\beta$ is the phase constant of the line and $R p$ is the parallel resistance of the resonator. In the case of $\lambda / 4$ SWO, the value of $Z_{L}$ is zero and $l$ is $\lambda / 4$.

As can be seen from Figure 2.6, tapering the transmission line to minimize the line width toward $z=0$ and maximize it toward $z=l$, can optimize the $Q$ of the resonator and hence improve the phase noise based on (2.8). From this figure, a voltage maximum happens toward $z=0$ and minimizing the line width can reduce the effect of $G$. On the other hand, a current maximum happens toward $z=l$ and maximizing the line width will reduce the effect of $R$. 
Figure 2.7 shows the final transmission line structure used in [18]. Measurement results of this reference show $8 \mathrm{~dB}$ of phase noise improvement at a $1 \mathrm{MHz}$ offset from the $15 \mathrm{GHz}$ carrier frequency, using the tapered transmission line technique. The measured phase noise value of this design is $-110 \mathrm{dBc} / \mathrm{Hz}$.

Even though [18] shows an interesting method to enhance the $Q$ of the SWO's resonator in order to improve the phase noise, the design work and layout of this reference is quite complex even for a simple $\lambda / 4$ structure. Adding more gain cells to the resonator in order to compensate for the resonator's loss [20], can make the layout even more complex. This is due to the fact that using this approach, the characteristic impedance of the resonator should stay constant and this is not an easy task with a tapered resonator being loaded with multiple gain cells.

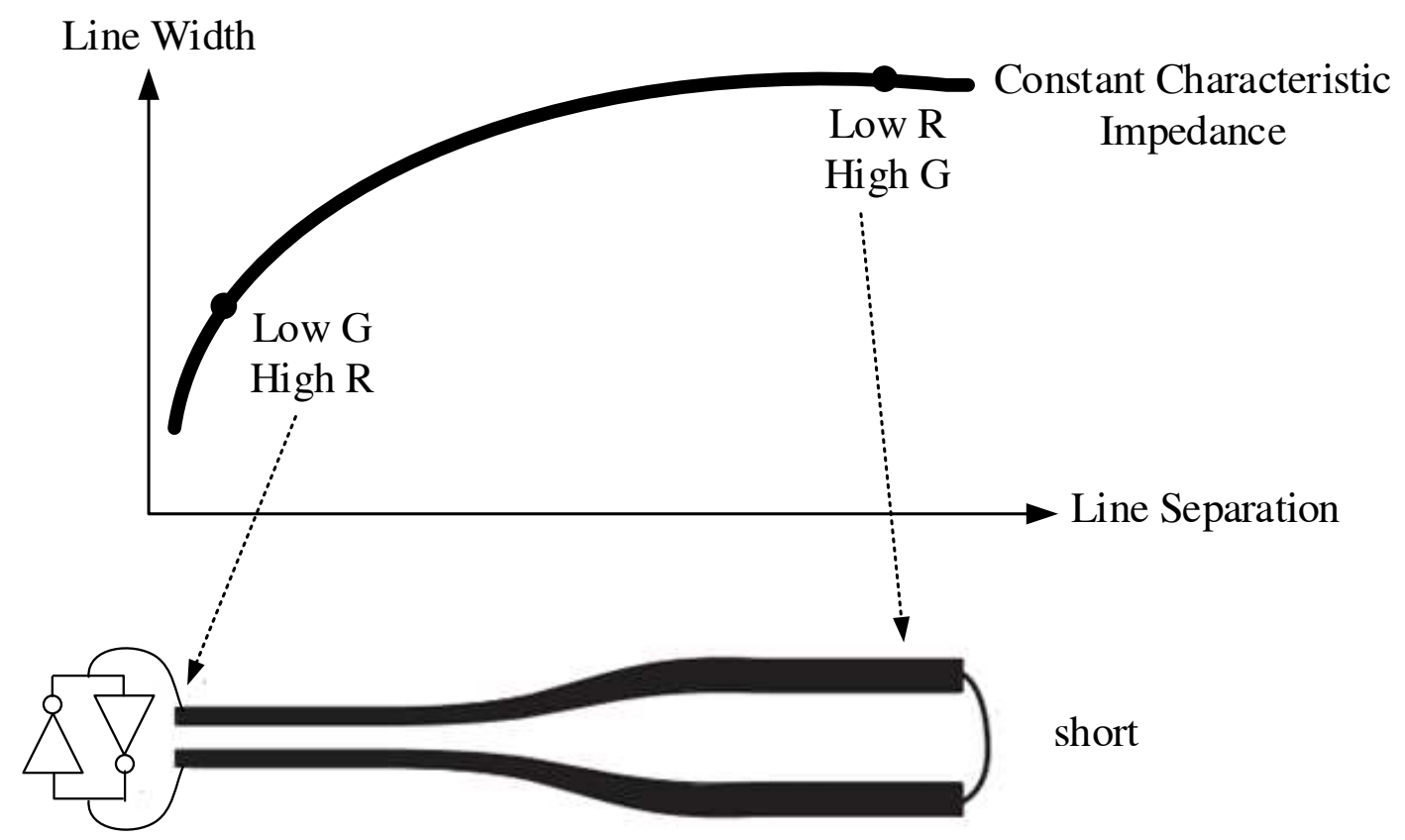

Figure 2.7 $\quad \lambda / 4$ SWO using a tapered CPS [18] 
Reference [21] shows a $27 \mathrm{GHz}$ SWO design which achieves a phase noise as low as $-115 \mathrm{dBc} / \mathrm{Hz}$ at a $1 \mathrm{MHz}$ offset from the carrier frequency. In this reference, a folded microstrip structure over floating metal strips has been used for the realization of the resonator. However, there is always a design tradeoff between the phase noise, size and power consumption of an oscillator. As a result, we can see higher power consumption and die area for [21] compared to [18], while [21] shows lower phase noise at an even higher frequency.

Researchers are employing different topologies in order to realize transmission-linebased resonators. However, there are unavoidable tradeoffs between the power consumption, size, complexity and phase noise of these oscillators.

In order to generate high frequency $I / Q$ signals for clock distribution or to design a high frequency phased-array system, multiple output phases are required and the SWOs are not a practical option for such applications. As a result, other wave-based oscillators like Travelling-wave Oscillators (TWO) or Rotary Travelling-wave Oscillators (RTWO) which are capable of generating multiple output phases are worth being studied.

\subsubsection{Travelling-wave Oscillators (TWO)}

A simple TWO can be formed by feeding the output of a distributed amplifier back to its input. Once the oscillation starts, based on (2.1) and (2.2), the signal travels only in one direction and tapping off from different positions on the resonator gives access to multiple output phases. 
Figure 2.8 shows a four-stage cascode, resistively terminated distributed amplifier [22] which is used to realize a TWO by adding a feedback between the input (node 1) and output (node 2) of the amplifier.

The gain of a distributed amplifier, if both drain and gate lines have the same propagation constants, can be calculated based on (2.11) [23].

$$
A_{v}=\frac{V_{2}}{V_{1}}=-N g_{m}\left(Z_{L} \| Z_{d}\right) e^{-N \alpha l} e^{-j N \beta l}
$$

where $N$ is the number of gain stages, $l$ is the physical distance between two gain stages, $g_{m}$ is the transconductance of the cascode transistors, $Z_{L}$ is the loading impedance and $Z_{d}$ is the drain line characteristic impedance, while $\alpha$ and $\beta$ are the attenuation and phase constant of the artificial transmission lines, respectively.

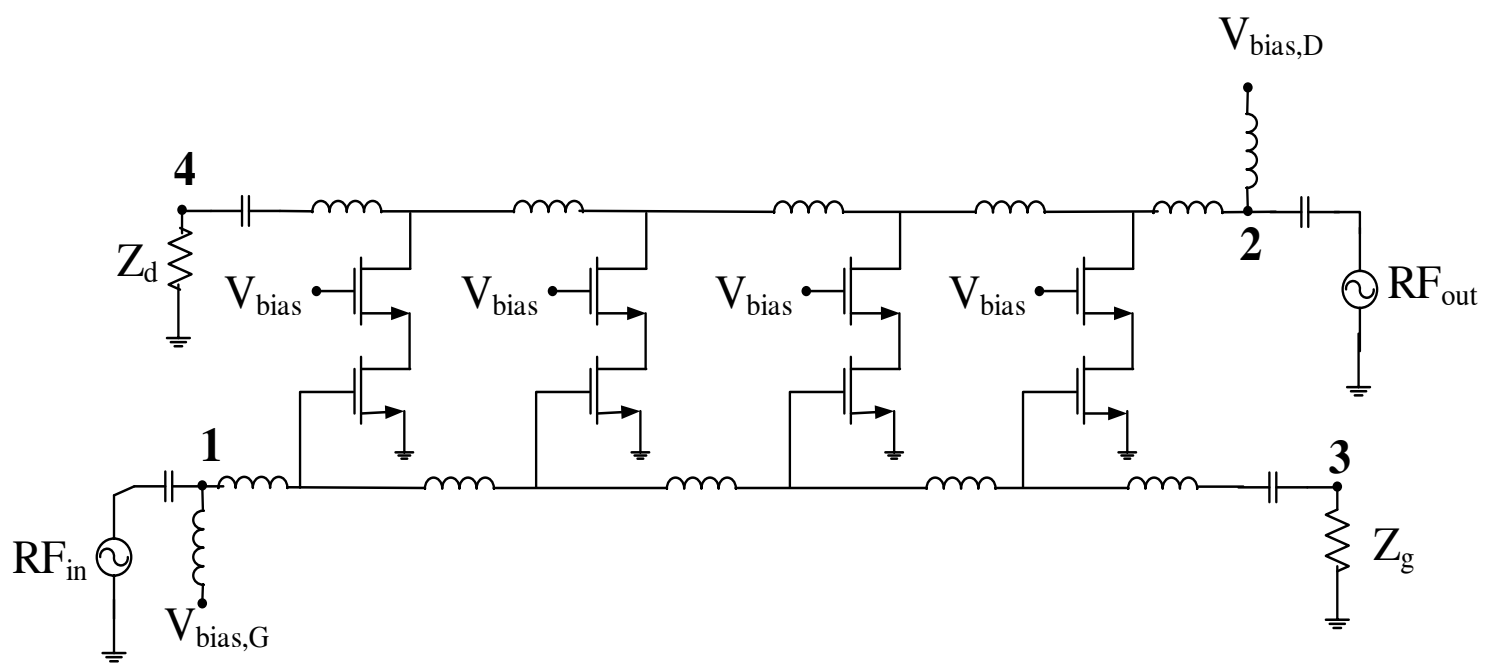

Figure 2.8 Four-stage resistively terminated distributed amplifier 
Applying a feedback to the distributed amplifier shown in Figure 2.8, can convert it to a travelling-wave oscillator, seen in Figure 2.9, with a predictable oscillation frequency and output power.

Based on the Barkhausen criteria, the large signal gain of the oscillator should be greater than unity. Applying this criterion to (2.11), the oscillation frequency can be calculated based on the large-signal transconductance, $G_{m}$, of the gain stages and based on (2.12).

$$
A_{v}=-N G_{m}\left(Z_{g} \| Z_{d}\right) e^{-N \alpha l} e^{-j N \beta l}=1
$$

Due to the feedback path between nodes 1 and 2 of Figure 2.9, the loading impedance, $Z_{L}$, of the drain line will be equal to $Z_{g}$ which is the characteristic impedance of the gate line.

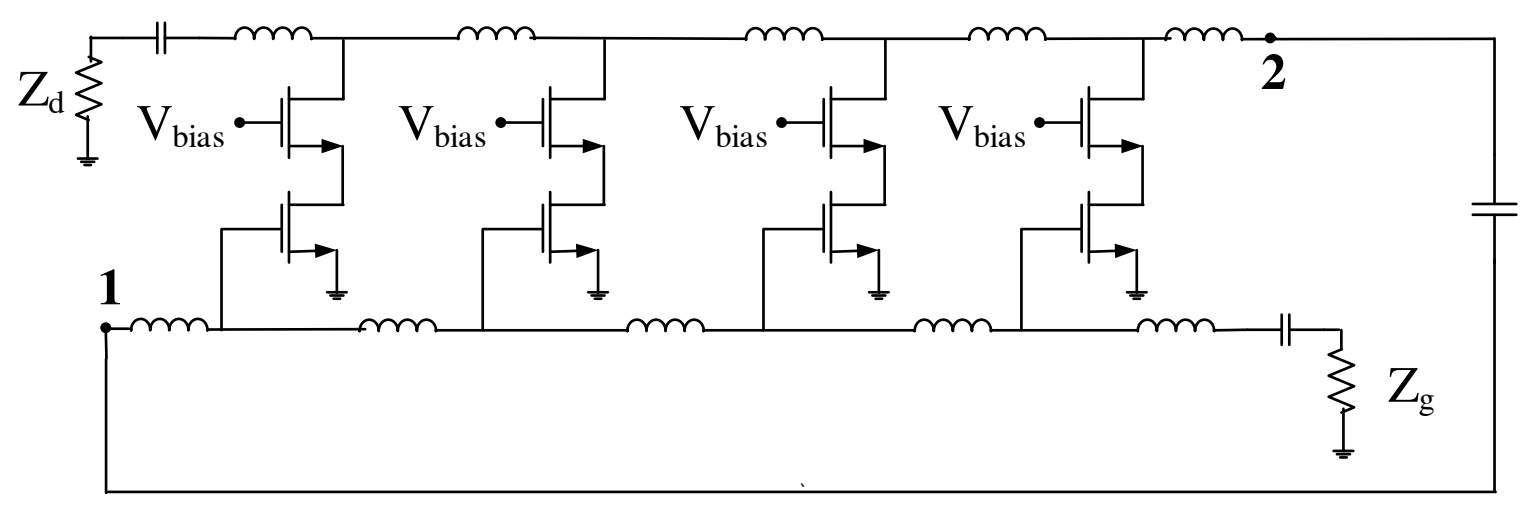

Figure 2.9 Distributed oscillator realized from a distributed amplifier 
Assuming purely real impedances for both $Z_{g}$ and $Z_{d}$, which are normally matched to the characteristic impedance of the drain and gate lines, the imaginary part of the left side of (2.12) should be equal to one.

(2.13) - (2.14) shows the oscillation frequency of the TWO configured from the shown distributed amplifier in Figure 2.8.

$$
\begin{gathered}
e^{-j N \beta l}=-1 \rightarrow e^{-j N \beta l}=e^{-j \pi} \\
\beta=\frac{2 \pi f_{\text {osc }}}{v_{\text {phase }}}=\frac{\pi}{N l} \\
v_{\text {phase }} \approx \frac{1}{\sqrt{L_{\text {perlength }} C_{\text {perlength }}}}
\end{gathered}
$$

where $v_{\text {phase }}$ is the phase velocity, $L_{\text {perlength }}$ and $C_{\text {perlength }}$ are the per-unit-length inductance and capacitance of the loaded lines, respectively.

As a result, the oscillation frequency of the TWO is:

$$
f_{\text {osc }} \approx \frac{1}{2 N l_{\text {segment }} \sqrt{L_{\text {perlength }} C_{\text {perlength }}}}
$$

where $l_{\text {segment }}$ is the physical length between each two gain stages.

The cut-off frequency of an artificial transmission line is [23]:

$$
f_{\text {bragg }}=f_{\text {cut-off }}=\frac{1}{\pi l_{\text {segment }} \sqrt{L_{\text {perlength }} C_{\text {perlength }}}}
$$

The cut-off frequency is the frequency at which no signal travels along the transmission line. 
Assuming both gate and drain lines have the same cut-off frequencies, and comparing (2.14) to (2.15), we can find a relationship between the oscillation frequency of the TWO, the number of gain cells, and the cut-off frequency of the distributed amplifier forming the TWO.

$$
\frac{f_{c u t-o f f}}{f_{o s c}}=\frac{2 N}{\pi}
$$

From (2.16), a typical distributed amplifier with four gain stages can oscillate at $39 \% f_{\text {cut-off, }}$ after feeding the output of the amplifier back to its input. Clearly an alternative configuration is required if the oscillation frequency needs to be closer to the cut-off frequency [24].

From (2.16) it can be also concluded that as we increase the number of gain cells in the design of a TWO, the ratio of $f_{\text {cut-off }}$ to $f_{\text {osc }}$ gets higher and the oscillation frequency gets closer to the predicted frequency of (2.14). Higher cut-off frequency can also lead to a phase noise improvement which will be explained in detail in Chapter 3.

Reference [25] and [26], have configured TWOs from resistively terminated distributed amplifiers and in both cases, the TWO is formed based on adding a feedback between input and output nodes of the distributed amplifier.

However, designing a TWO from a distributed amplifier utilizing big transistors to provide high voltage gain, perhaps is not the most efficient method since according to the Barkhausen criteria the overall voltage gain of the loop should be greater than unity and not the voltage gain of each stage, individually. 
The main disadvantage of TWOs configured from a simple resistively terminated distributed amplifier, regardless of the gain cell's topology, is the termination of the resonator which has a strong effect on the quality factor and consequently the phase noise of the oscillator [26].

The phase noise contribution of the tank of such TWOs at an offset frequency of $\omega_{m}$ can be calculated based on (2.17):

$$
\begin{gathered}
\mathcal{L}\left(\omega_{m}\right)_{\text {tank-TWO }}=\frac{k T Z_{0}}{V_{0}{ }^{2}}\left(\frac{\omega_{0}}{2 \omega_{m} Q}\right)^{2} \\
Q=\pi \\
V_{0} \approx I_{\text {tail }} Z_{0} e^{-0.5}
\end{gathered}
$$

where $Z_{0}$ is the termination impedance of the TWO which is normally matched to the characteristic impedance of the lines and $I_{\text {tail }}$ is the tail DC current of each gain cell.

In (2.17), $V_{0}$ is the oscillator's output voltage and we have calculated that based on the real part of (2.12), and the relationship between $Q, \alpha$ and $\beta$ where:

$$
Q=\frac{\beta}{2 \alpha}
$$

The phase noise equation in (2.17) has been derived based on the proposed approach of [26] and after being re-arranged based on Leeson's phase noise equation.

From (2.17), we can clearly state that maximizing the characteristic impedance of a TWO's resonator can improve the phase noise of the oscillator since the output voltage swing is increased which has quadratic effect of the phase noise reduction. 
In order to have a fair conclusion about TWOs formed from distributed amplifiers, we should also mention that a very wideband distributed oscillators can be designed with the aid of active termination networks like, a common emitter transistor [27], cascodeconnected transistors [28] or $R L$ terminating network [29]. Replacing the resistive termination of these structures with other active or passive components can improve the phase noise performance of the configured distributed oscillator. However, the phase noise contribution of the TWO's resonator is still affected by its termination network.

Comparing TWOs and SWOs, the main advantage of a TWO is the fact that it is generating multiple output phases at very high frequencies. However, TWOs are all suffering from their termination networks. The resonator of TWOs is not fully closedloop and part of the signal is wasted in the termination. This is why TWOs normally need gain stages with higher transconductance compared to SWOs. Furthermore, termination networks of a TWO, regardless of its topology, are constant noise sources which degrade the overall phase noise of the TWO.

As explained earlier, SWOs have a completely closed-loop resonator without any termination and they show a superior phase noise performance compare to TWOs. However, they are single-phase structures and are not practical if multiple output phases are required. 


\subsubsection{Rotary Travelling-Wave Oscillator (RTWO)}

A Rotary Travelling-Wave Oscillator (RTWO) can be formed by adding one more feedback, from (node 3) to (node 4) shown in Figure 2.10, to the TWO shown in Figure 2.9 and it can be neatly analyzed based on the superposition of multiple $\lambda / 2$ SWOs.

Figure 2.10 shows an RTWO formed from a distributed amplifier after adding the second feedback to the circuit. Like the TWO, since the signal propagates in one direction along the transmission line, multiple output phases are easily available by tapping off at different positions on the resonator.

In general, an RTWO is composed of an odd number of cross-connections of the inner and outer conductors to cause signal inversion. As a result, if the resonator is lossless, a wave could travel on the ring indefinitely, and provide a full clock cycle in every other rotation around the ring. However, since in real applications the resonator is lossy, multiple gain stages should be added to the ring to overcome the loss of the resonator and sustain the oscillation.

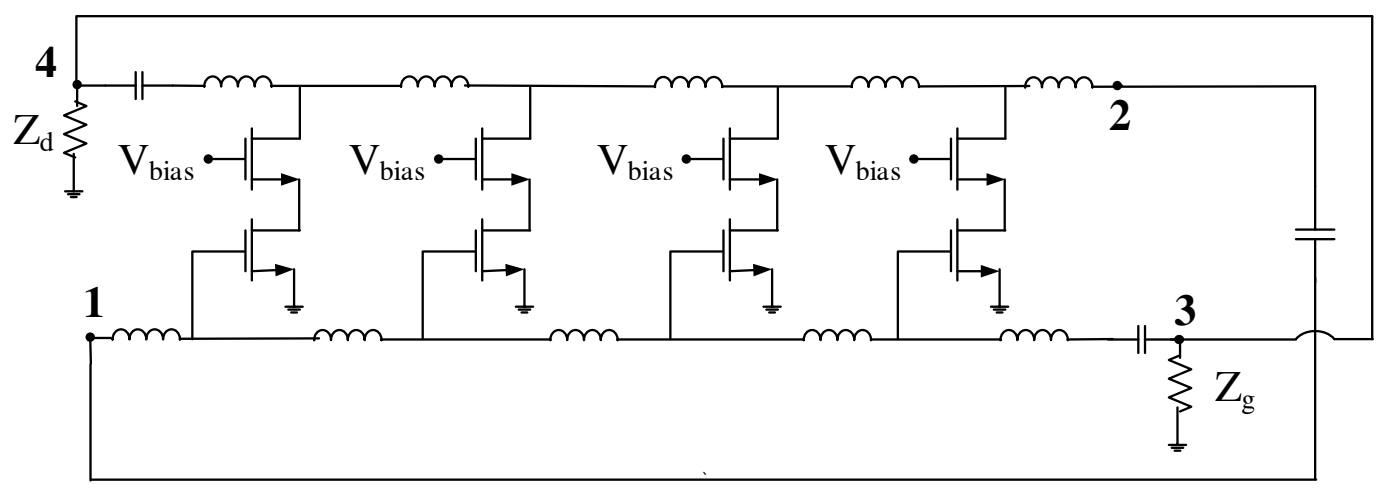

Figure 2.10 RTWO formed from a distributed amplifier 
The RTWO was first proposed by John Wood et al. in 2001 [1]. In this reference, cross-coupled CMOS inverters, or $-g_{m}$ cells, have been used as gain cells and a coplanar stripline with one cross-over structure is employed to realize the resonator.

As shown in this reference, the oscillation frequency of an RTWO is the same as the oscillation frequency of a TWO and it is determined based on the round-trip time delay because of the signal inversion. The RTWO's oscillation frequency is [1]:

$$
f_{\text {osc }}=\frac{1}{2 N l_{\text {segment }} \sqrt{L_{\text {perlen }} \cdot\left(C_{0}+\frac{C_{\text {seg }}}{l_{\text {seg }}}\right)}}
$$

where $l_{\text {segment }}$ is the physical length between each two segments, $L_{\text {perlen }}$ is the per-unitlength differential inductance of the transmission line, $C_{0}$ is the per-unit-length differential capacitance of the interconnect and $C_{\text {seg }}$ is the total differential capacitance of each cell of the VCO core.

Even though reference [1] has proposed the idea of the RTWO design for the first time, the paper focuses mainly on the clock distribution at $965 \mathrm{MHz}$ and not necessarily on oscillation frequency, tuning capability or the phase noise of an RTWO.

Later on, reference [30] has added varactors to an RTWO to generate $I / Q$ signals at $18 \mathrm{GHz}$, and provide some tuning range.

However, in this reference the RTWO gives access to only two output phases with $90^{\circ}$ of phase shift between them, and the geometry of the resonator is not fully 
symmetrical. This asymmetry can introduce significant amount of phase error if smaller phase steps at higher frequencies are required.

Reference [31] has shown how the transmission line design can affect the performance of the RTWO. In this paper, an exotic loop geometry, shown in Figure 2.11, has been designed which includes only one cross-over structure while it keeps the constant length between each two amplifying stages to minimize the phase error.

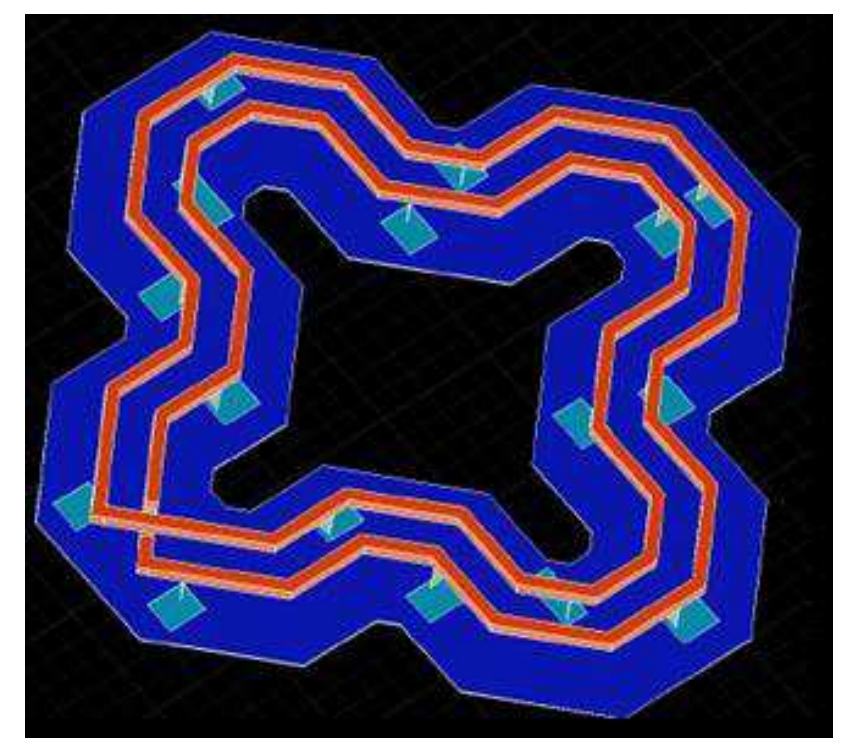

Figure 2.11 Symmetric resonator geometry with one cross-over [31]

However, the loop geometry includes so many bends and discontinuities and moving toward even higher frequencies needs very careful and time consuming EM simulations to take the effect of the discontinuities into account at every stage of the design. Furthermore, the inner and outer loop dimensions in this design are not the same and this is why the tapping points of differential outputs are not always located opposite each other, as seen in Figure 2.11. This can add error to the RTWO output phases since the 
gain cells should be connected to the loop with different-length interconnects. This effect is much more visible at higher frequencies, where the total length of the loop is greatly reduced and two tapping points of the same gain cell can be located very far from each other.

A detailed study of the phase noise analysis of a CMOS-based RTWO has been done in literature. Reference [19] has analyzed the RTWO as a superposition of multiple $\lambda / 2$ SWOs which in our opinion, is the best way to understand the performance of an RTWO.

Under a low-loss approximation, the number of harmonics, $n_{h}$, that a distributed line can sustain can be determined based on (2.20) [19],

$$
n_{h}=\left\lfloor\frac{N}{\pi}-\frac{1}{2}\right\rfloor
$$

where $N$ is the number of gain cells distributed across the resonator.

This equation is derived based on (2.16) and knowing that no harmonics can be sustained on the line above the cut-off frequency:

$$
\left(2 n_{h}+1\right) f_{o s c}<f_{\text {cut-off }}
$$

According to the Leeson's equation, seen in (2.22), the phase noise of an oscillator also depends on the noise factor, $F$, of the gain cells.

$$
\mathcal{L}\left(\omega_{m}\right)=\frac{4 F k T R_{p}}{V_{0}{ }^{2}}\left(\frac{\omega_{0}}{2 \omega_{m} Q}\right)^{2}
$$


The noise factor, $F$, of a CMOS-based oscillator using $-g_{m}$ topology in current limited region can be simplified to (2.23) [19]:

$$
\begin{gathered}
F=1+\gamma \frac{4 I_{\text {tail }} R_{p}}{\pi V_{0}\left(n_{h}+1\right)} \\
=1+\frac{\gamma}{n_{h}+1}
\end{gathered}
$$

where $\gamma$ is the channel noise coefficient of a FET device and $n_{h}$ is he number of harmonics.

In the $L C$-tank oscillator, where the harmonic voltages are significantly attenuated due to the filtering effect of the tank, the value of $n_{h}$ in (2.23) can be approximated by zero and the value of $F$ would be $1+\gamma$. In the SWO and RTWO, on the other hand, the resonator can sustain harmonics as well which improves the phase noise performance of distributed-based oscillators compared to $L C$-tank oscillators.

From (2.20) - (2.23), we can state that as we add more gain cells, while maintaining the frequency, to a transmission-line-based oscillator or consequently loading the line more heavily while keeping the oscillation frequency constant, we can improve the phase noise of an oscillator. From [19] the total phase noise of an RTWO utilizing CMOS $-g_{m}$ gain cells at an offset frequency of $\omega_{\mathrm{m}}$ can be calculated based on (2.24). More details on RTWO phase noise analysis will be provided in Chapter 3.

$$
\begin{gathered}
\mathcal{L}\left(\omega_{m}\right)_{R T W O}=\frac{2 F k T R_{p}}{V_{0}^{2}}\left(\frac{\omega_{0}}{2 \omega_{m} Q}\right)^{2} \\
Q=\frac{\pi^{2} R_{P}}{2 L \omega_{0}}
\end{gathered}
$$




$$
V_{0}=\frac{2 I_{\text {tail }} R_{p}}{\pi}
$$

where $R_{p}$ is the parallel resistance of the resonator, $V_{0}$ is the oscillator's output voltage, $I_{\text {tail }}$ is the total tail DC current and $Q$ is the quality factor of the resonator.

Table 1 summarizes the main characteristics of the three different transmission-linebased oscillators discussed in this chapter. In this table, the available number of output phases, oscillation frequency, resonator quality factor and the phase noise contribution of the tanks of the SWOs, resistive terminated TWOs and RTWOs are compared.

The total phase noise of any of the mentioned oscillators depends on the gain stage and tail current topologies used in their designs and a comparison between them is not valid unless the complete circuit is available. As a result, we have only included the resonator's phase noise contribution of each of these oscillators in this table.

Table 1 Comparison of SWOs, TWOs and RTWOs

\begin{tabular}{|c|c|c|c|c|}
\hline $\begin{array}{c}\text { Oscillator } \\
\text { Topology }\end{array}$ & $\begin{array}{c}\text { Number of } \\
\text { Output } \\
\text { Phases }\end{array}$ & $\begin{array}{c}\text { Oscillation } \\
\text { Frequency }\end{array}$ & $\begin{array}{c}\text { Resonator } \\
\text { Quality Factor }\end{array}$ & $\begin{array}{c}\text { Phase Noise } \\
\text { Contribution of the } \\
\text { Resonator }\end{array}$ \\
\hline $\boldsymbol{L C}$-tank & $0^{\circ}, 180^{\circ}$ & $\frac{1}{2 \pi \sqrt{L_{\text {total }} C_{\text {total }}}}$ & $\frac{R_{p}}{L \omega_{0}}$ & $\frac{4 k T R_{p}}{V_{0}{ }^{2}}\left(\frac{\omega_{0}}{2 \omega_{m} Q}\right)^{2}$ \\
\hline N4 SWO & $0^{\circ}, 180^{\circ}$ & $\frac{1}{2 \sqrt{L_{\text {total }} C_{\text {total }}}}$ & $\frac{\pi^{2} R_{P}}{8 L \omega_{0}}$ & $\frac{4 k T R_{p}}{V_{0}^{2}}\left(\frac{\omega_{0}}{2 \omega_{m} Q}\right)^{2}$ \\
\hline TWO & multiple & $\frac{1}{\sqrt{L_{\text {total }} C_{\text {total }}}}$ & $\frac{k T Z_{0}}{V_{0}^{2}}\left(\frac{\omega_{0}}{2 \omega_{m} Q}\right)^{2}$ \\
\hline RTWO & multiple & $\frac{1}{\sqrt{L_{\text {total }} C_{\text {total }}}}$ & $\frac{\pi^{2} R_{P}}{2 L \omega_{0}}$ & $\frac{2 k T R_{p}}{V_{0}{ }^{2}}\left(\frac{\omega_{0}}{2 \omega_{m} Q}\right)^{2}$ \\
\hline
\end{tabular}


As seen in Table 1, among different types of transmission-line-based oscillators, the RTWO has the unique property of giving access to multiple output phases at high frequencies while based on (2.22) to (2.24), the phase noise of an RTWO can be even better than that of the $L C$-tank oscillator if more than four gain stages are employed in its design.

The other interesting insight from this table is the comparison between the oscillation frequencies of different oscillators. From this table, for the same value of $L_{\text {total }}=2 n l L_{\text {perlen }}$ and $C t_{\text {otal }}=2 \mathrm{nl} C_{\text {perlen}}$, the oscillation frequency of TWO and RTWO is $2 \pi$ higher than the $L C$-tank oscillator!

\subsection{Phased-array Principles}

Recent advances in CMOS and BiCMOS technologies make it possible to integrate full complex transceivers on a silicon substrate. In phased-array receivers, the noise figure and SNR can be improved by implementing multiple antennas to receive the transmitted signal. Meanwhile, one of the main challenges of integrated high frequency transmitters is the design of high output power, high efficiency power amplifiers (PAs).

However, phased-array transmitters can effectively combine the power of multiple PAs in space, and in addition they can form a beam in a desired direction electronically.

In order to design an integrated phased-array transmitter, a component which can create uniform phase shift either in the Intermediate Frequency (IF) [7], Radio Frequency 
(RF) [32], [33] or Local Oscillator (LO) [9], [34], [35] path is required. Figure 2.12 shows block diagrams of phased-array transmitters showing different architectures.

IF phase shifting, Figure 2.12(a), could be beneficial since the phase shifting action can be done at base-band where the frequency is quite low and signal distribution to different antennas is not sensitive to any length mismatch. However, IF phase shifting for designing phased-array transmitters requires multiple phase shifters to create constant phase shifts depending on the number of required phases and also multiple up-conversion mixers are required to mix the signal up to RF frequency. Furthermore, the LO signals which are driving the switching transistors of up-conversion mixers must be locked to the same phase otherwise they can directly add to the phase error of the system.

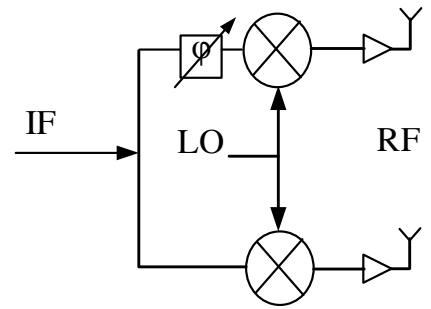

(a)

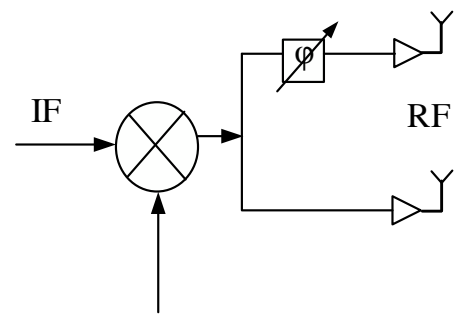

LO

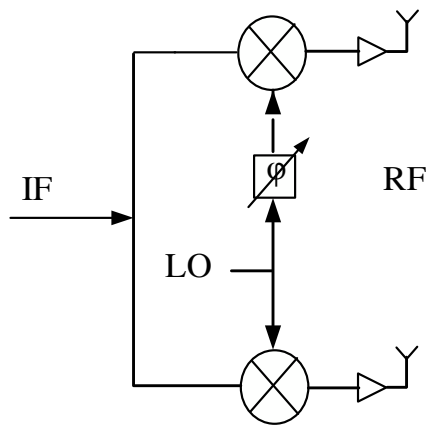

(c)

Figure 2.12 Phased-array architectures (a) IF phase shift (b) RF phase shift (c) LO phase shift

Comparing IF phase shifting to RF phase shifting, we can state that in the second method, shown in Figure 2.12(b), only one mixer with a single-phase LO is needed. However, this method suffers from the need of multiple variable-gain amplifiers to compensate for the RF amplitude mismatches between the signals. Furthermore, like the 
IF phase shifting method, multiple phase shifters are mandatory to create constant phase shifts.

Finally LO phase shifting, Figure 2.12(c), requires an oscillator which provides multiple output phases to drive the up-conversion mixers. However, since the mixers are less sensitive to amplitude mismatch of LO signals compared to RF signals, variable-gain amplifiers are not needed with this method. The main drawback of LO phase shifting is the requirement of the distribution network to deliver the output signals of the oscillator to different antennas while maintaining the phase. At high frequencies, the design of a distribution network can be very challenging.

Figure 2.13 shows a conceptual $n$-element phased-array transmitter utilizing an RTWO for LO phase shifting.

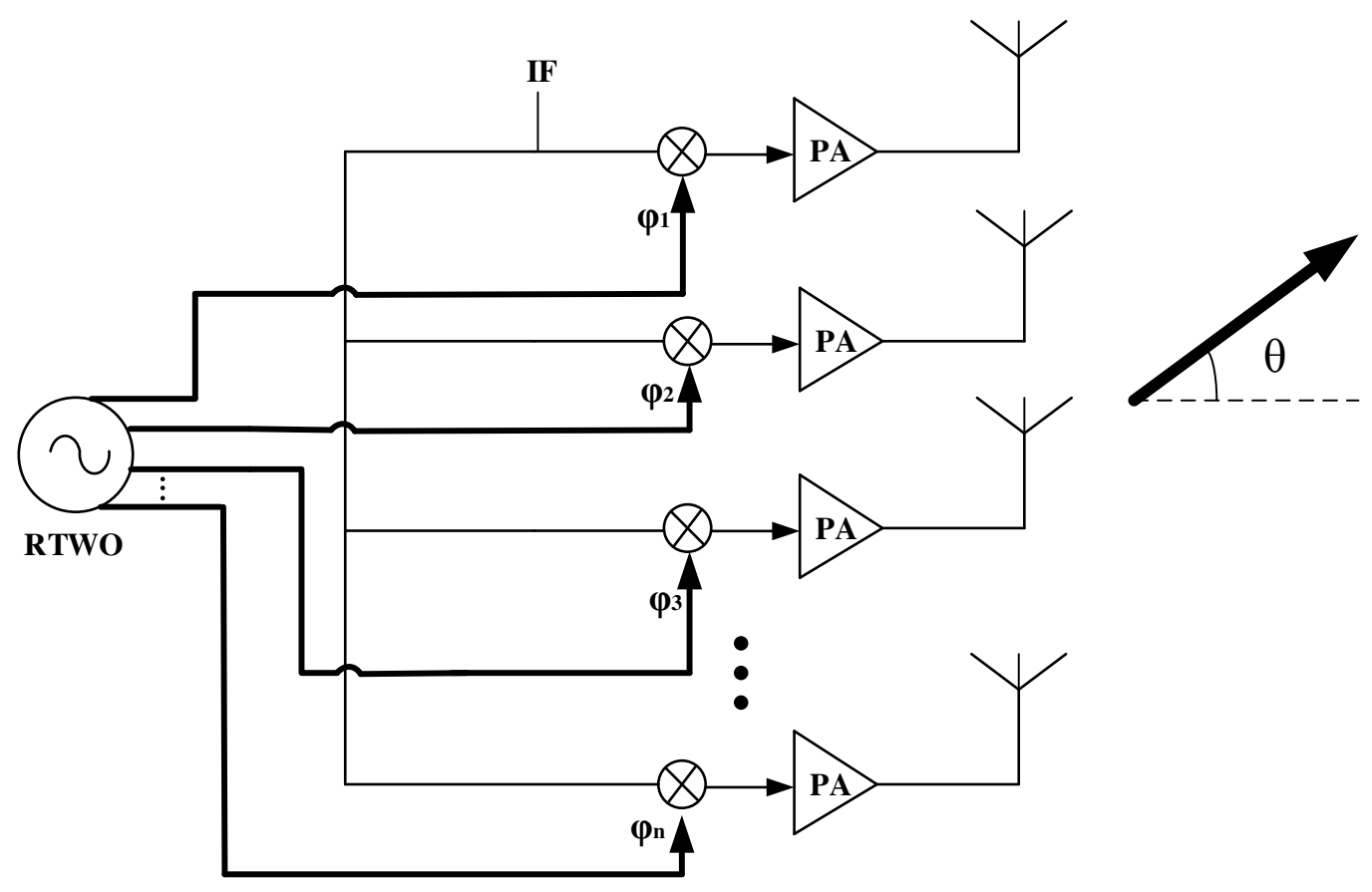

Figure 2.13 Conceptual $n$-element phased-array transmitter using an RTWO 
In this figure, the relative phase shift between the elements, $\varphi_{1}$ to $\varphi n$, defines the radiation angle, $\theta$, based on (2.25) [36],

$$
\theta=\sin ^{-1}\left(\frac{\varphi \cdot c}{\omega_{R F} d}\right)
$$

where $c$ is the velocity of light, $d$ is the spacing between the antennas and $\omega_{R F}$ is the radiated RF frequency in the space.

In case of $d=\lambda_{R F} / 2$ :

$$
\theta=\sin ^{-1}\left(\frac{\varphi}{\pi}\right)
$$

In an $n$-element array transmitter, if each element radiates $P$ watts of power, the effective isotropic radiated power (EIRP) in the desired direction is $n^{2} P$ watts [36]. This increase in signal power is quite beneficial for the design of high frequency transmitters since the design of a single high frequency, high output power and efficient PA is still challenging. However, in $n$-element array transmitters, the combination of multiple less efficient PAs can solve this issue since the power will be focused in the desired direction.

For this research work I have employed a Ku-band RTWO in a two-element phasedarray transmitter.

\subsection{Summary}

Clocking at gigahertz rates requires generators with low skew and low phase noise. However, as the frequency increases, it becomes increasingly difficult to achieve the 
desired specifications, using $L C$-tank resonators. Therefore, researchers have looked into alternative oscillator topologies and among them distributed oscillators seem to be the solution.

There are three main categories of distributed oscillators and among them, the RTWO has the unique property of sustaining a high output power travelling-wave while dissipating low power and achieving low phase noise at high frequencies. The resonator of this structure is fully closed-loop and its phase noise performance is comparable to the $L C$-tank oscillator while it can operate up to the cut-off frequency of its resonator with much less power consumption than the $L C$-tank oscillator.

The other multiphase distributed-based oscillator discussed in this chapter is a TWO. However, since the resonator of this structure is not fully closed-loop, part of signal is wasted in the termination network and the phase noise performance and output power of these oscillators is worse than RTWOs. For resistively terminated TWOs, the quality factor of the resonator is $\pi$ regardless of the geometry of the resonator.

Comparing RTWOs with standing-wave oscillators, an RTWO can provide multiple output phases, instead of only one differential phase as in SWO's case, with comparable amount of phase noise. However, the output voltage of an RTWO is half that of an SWO. The skew and jitter of distributed signals in a clock distribution network utilizing SWOs, can be significantly reduced. This is due to the fact that in SWOs the output phases are not depending on the position across the ring. 
One of the main applications of an RTWO is in phased-array transmitters which can effectively combine the power of multiple PAs in space, and electronically form the beam in a particular direction.

However, in order to design a phased-array transmitter and take advantage of its characteristic, a component which can create constant phase shift in either the IF, RF or LO path is needed. Among different methods of phase shifting to design phased-array transmitters, the LO phase shifting eliminates the need for multiple phase shifters and also variable gain amplifiers as long as the LO signal power is high enough to fully switch the mixer's switching transistors. Having access to an oscillator which can generate multiple output phases with high amplitude can heavily reduce the power consumption of the phased-array transmitter utilizing LO phase shifting. 


\section{Chapter 3 Design of a Ku-band}

\section{Rotary Travelling-wave Oscillator}

In this chapter, the analytical calculations, design considerations and layout implementation of a fully integrated eight-phase $17.5 \mathrm{GHz}$ rotary travelling-wave voltage-controlled oscillator (RTW-VCO) fabricated in $0.25 \mu \mathrm{m}$ BiCMOS process are presented.

The RTWO has been designed for Ku-band of operation and a phased-array transmitter application. The main focus of our design is accurate output phases for beamforming and the physical size of the RTWO.

\subsection{The RTWO Architecture}

An RTWO is composed of a differential resonator with an odd number of cross-over structures for the signal inversion. An even number of gain stages should be distributed across the resonator to compensate for the resonator's loss and to sustain the oscillation in a traveling-wave mode.

In order to design an eight-phase RTWO, we have employed four stages of crosscoupled NPN transistors which are uniformly distributed along a coupled stripline ring to both overcome the loss of the resonator and impose the odd mode of operation. 
Figure 3.1 shows the cross-coupled NPN transistors operating as gain stages in our RTWO design.

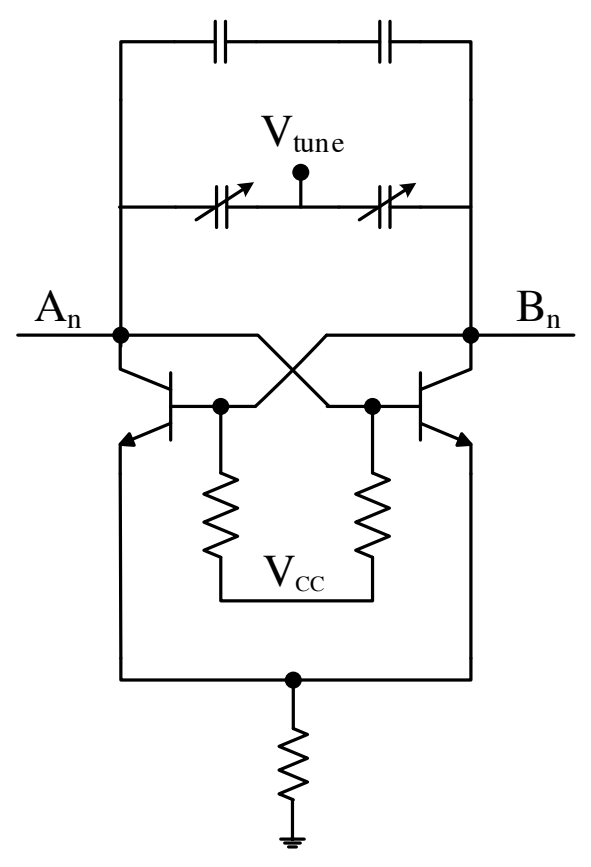

Figure 3.1 Cross-coupled NPN transistors forming a $-g_{m}$ topology

This topology has been used because of its simplicity. It is also inherently differential which enforces the odd mode of operation. In this design, in order to have more headroom, the tail current source of normal $-g_{m}$ cells is replaced with a $140 \Omega$ resistor.

As seen in Figure 3.1, differential NMOS varactors are used in this design in order to tune the oscillation frequency which can drift due to any process, temperature and power supply variation. Two $45 \mathrm{fF}$ MIM capacitors are used in parallel with the varactors to lower the gain of the $\mathrm{VCO}, K_{V C O}$, for sensitivity purposes.

Figure 3.2 shows the capacitance ratio and quality factor, $Q$, of the varactors versus the tuning voltage from $0 \mathrm{~V}$ to $2.5 \mathrm{~V}$. 
As illustrated in this figure, the differential $Q$ of the varactor at $18 \mathrm{GHz}$ is quite high and it varies from 24 to 62 while the differential capacitance changes from $52 \mathrm{fF}$ to $22 \mathrm{fF}$ for the same tuning voltage. This relatively high value of quality factor is achieved after running a few simulations in order to find the optimum size of the varactors.
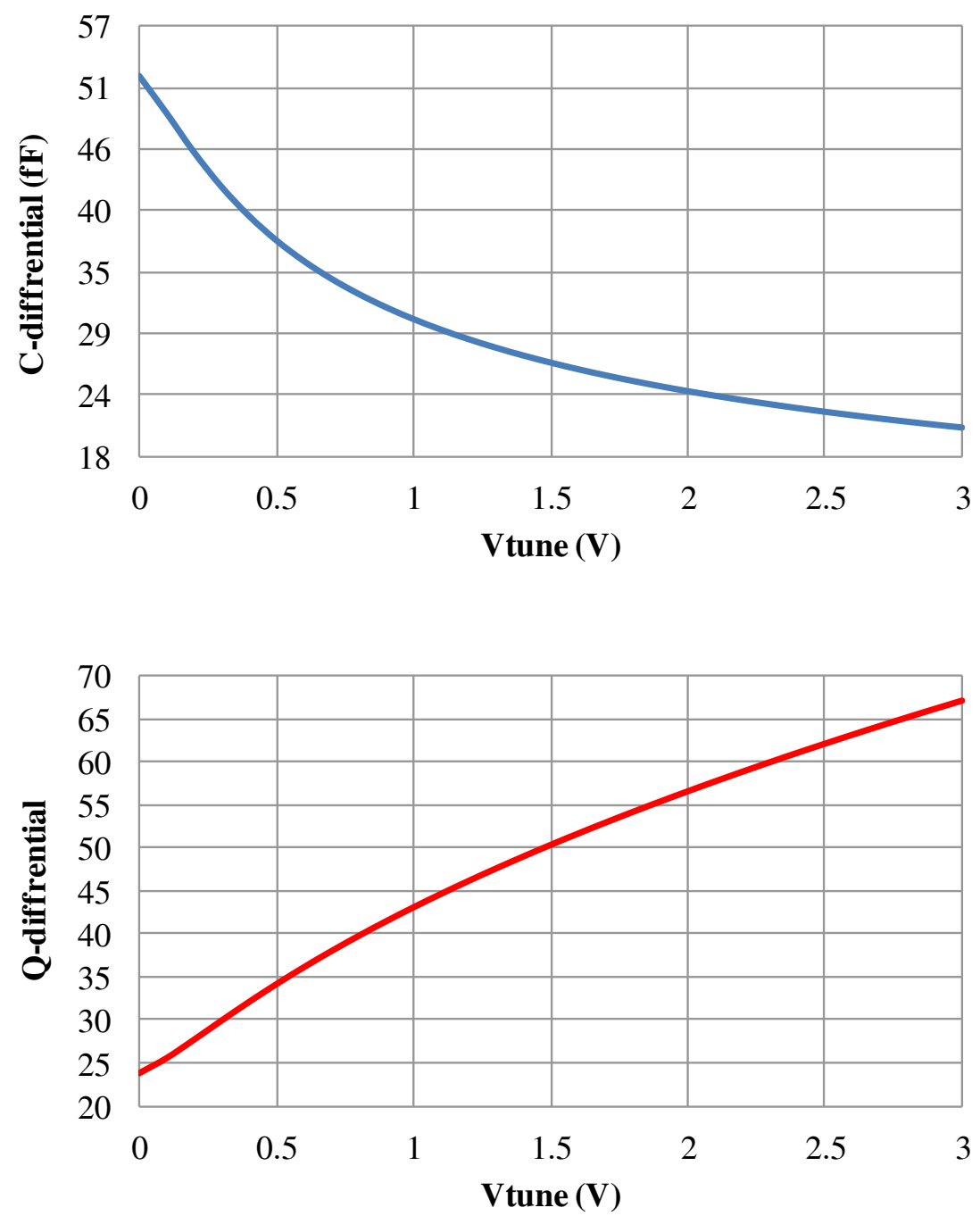

Figure 3.2 Characterization of the differential NMOS varactors at $18 \mathrm{GHz}$

Our resonator is realized by microstrip-line structures with a signal line being laid out using the $3 \mu \mathrm{m}$ thick Aluminum layer $\left(M_{6}\right)$ on the top and the first metal layer $\left(M_{1}\right)$ used 
as a ground plane on the bottom to eliminate the non-ideal effects of the lossy silicon substrate. The total length of the resonator is $1.488 \mathrm{~mm}$. The width and spacing of the differential microstrip lines are $7 \mu \mathrm{m}$ and $68 \mu \mathrm{m}$, respectively. The vertical distance between the top metal layer to the first metal layer is $8.1 \mu \mathrm{m}$ in this process.

As discussed in Chapter 2, different topologies can be employed in order to design the resonator of wave-based oscillators. Each of these topologies has some advantages and disadvantages and they are beneficial for some particular applications, otherwise there is always a design tradeoff between the $Q$, complexity and size of the resonator.

In this work, the resonator has been designed to provide maximum symmetry while it is not complex and bulky. The distance between all tapping points in our design is the same and equal to $186 \mu \mathrm{m}$, thanks to the geometry of the resonator.

We have used three cross-overs of the inner and outer lines for the realization of the resonator while all these cross-over structures are implemented with an orthogonal structure instead of a diagonally perpendicular structure resulting in all tapping points being exactly the same distance apart. This advantage is not seen with the diagonally perpendicular structure used in [37] - [38].

Figure 3.3 shows the schematic diagram of our complete RTW-VCO. Two emitterfollower buffer stages have been added to all $A n+1$ and $B n+1$ nodes seen in Figure 3.3. The reverse isolation of the buffers is $-22 \mathrm{~dB}$ which is adequate for protecting the core VCO from loading and frequency pulling. 


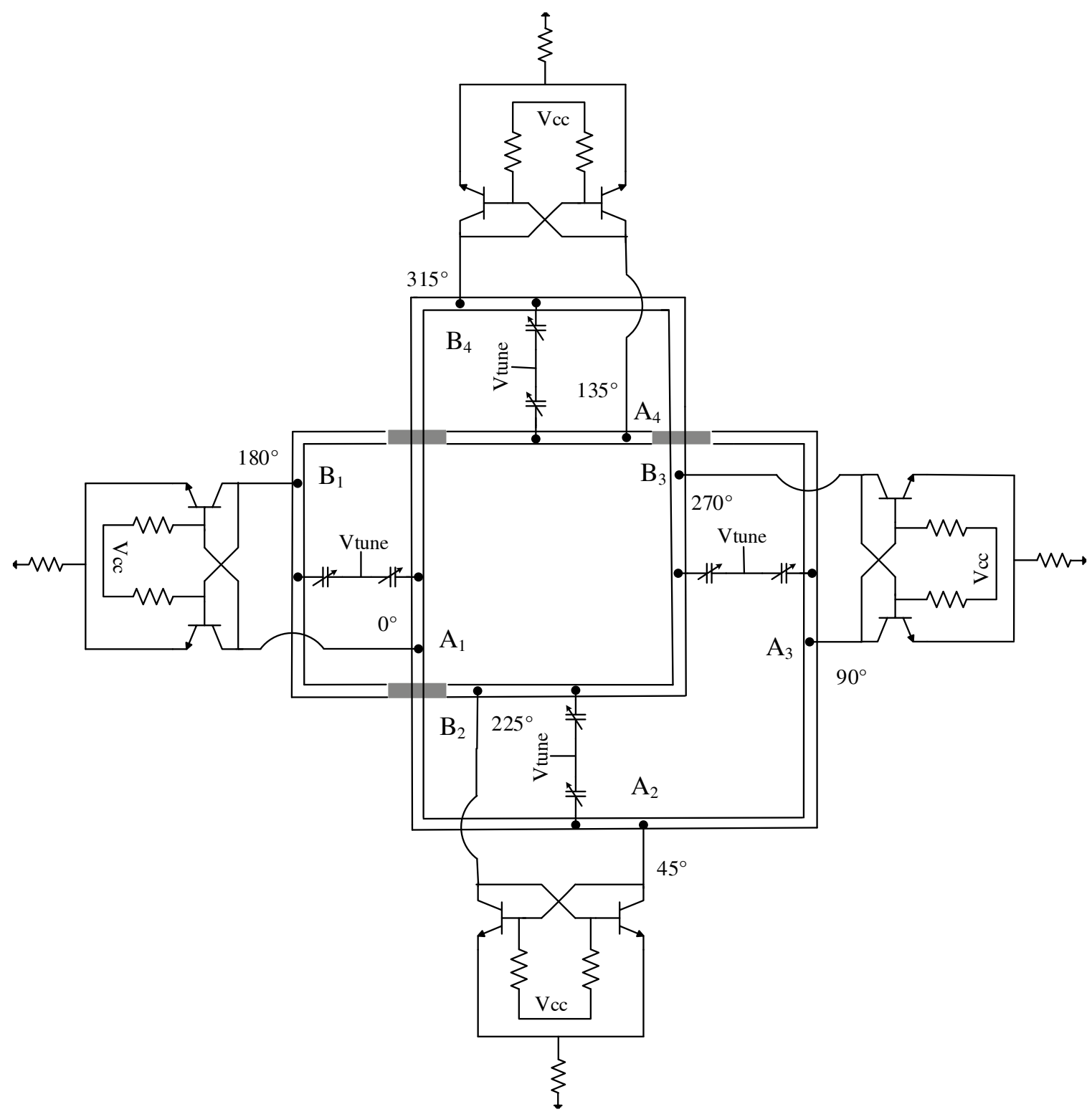

Figure 3.3 Schematic diagram of the proposed RTW-VCO

\subsubsection{Design Methodology}

In this section, the analytical model of each RTWO segment used to select component values in order to predict the oscillation frequency, output power and phase noise is presented. 
Figure 3.4 shows the RF model of one segment of the RTW-VCO with all RF components and parasitic capacitances of the NPN transistors. This model can also be used as a $R L C G$ model of the resonator in Cadence simulations in order to ease the convergence, if needed.

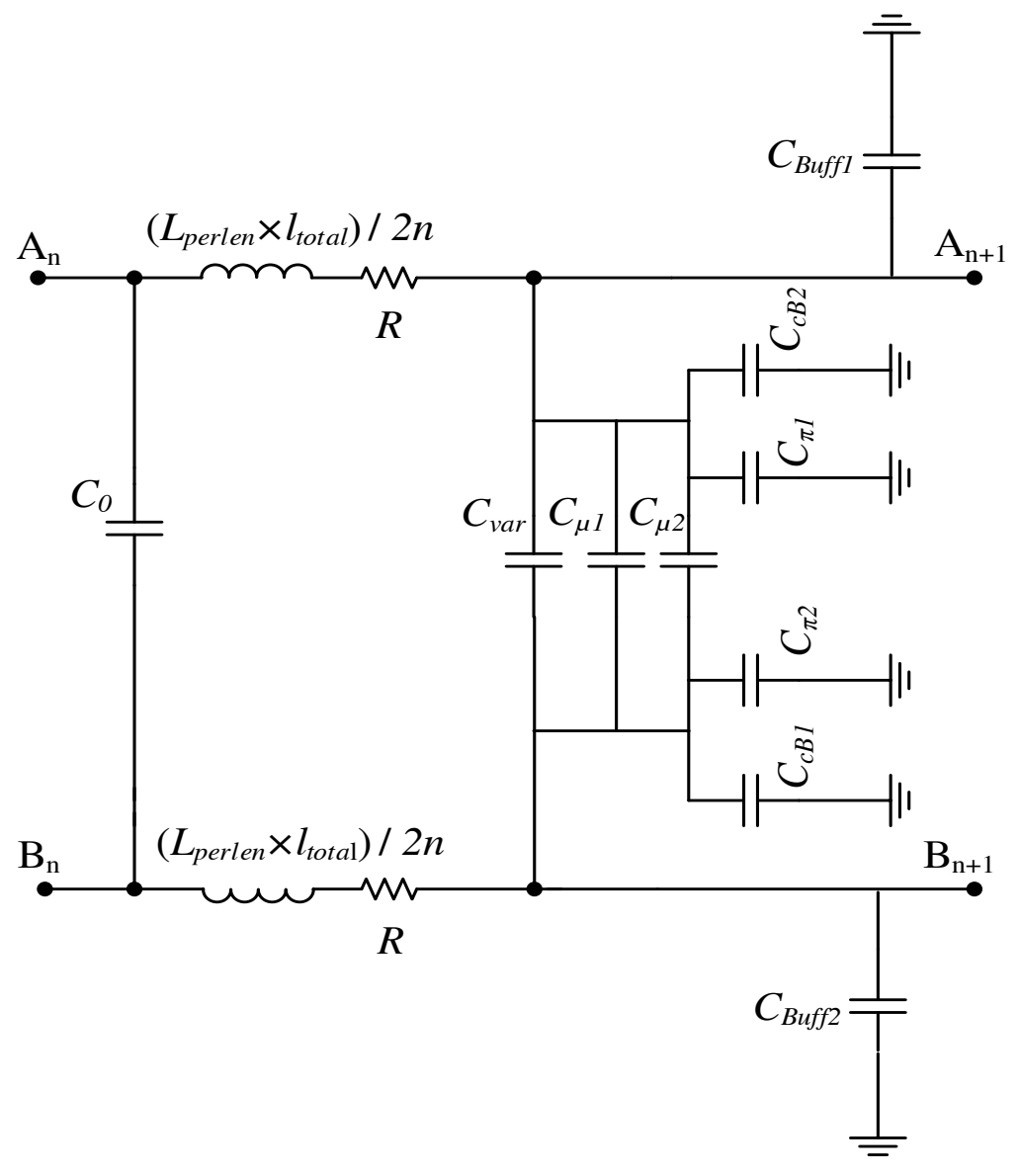

Figure 3.4 The RF model of one segment of the RTW-VCO

The oscillation frequency of an RTWO is determined based on the round-trip time delay, due to the signal inversion, and can be calculated from (2.19).

From Figure 3.4, the differential parasitic capacitance of each segment can be calculated as follows: 


$$
C_{s e g}=\left(C_{\mu 1}+C_{\mu 2}+C_{v a r}+\frac{\left(C_{\pi 1}+C_{\pi 2}+C_{c B 1}+C_{c B 2}+C_{B u f f 1}+C_{B u f f 2}\right)}{4}\right)
$$

where $C_{\mu}$ is the base to collector capacitance, $C_{v a r}$ is the differential varactor capacitance at a particular bias point, $C_{\pi}$ is the base to emitter capacitance, $C_{c B}$ is the collector to bulk capacitance and $C_{B u f f}$ is the total parasitic capacitance of each buffer stage.

In order to calculate the oscillation frequency of the RTWO, we also need to have an estimate for the differential inductance and capacitance of the resonator.

Since we have used a big gap of $68 \mu \mathrm{m}$ between the inner and outer lines, the mutual inductance between the lines has been ignored in our theoretical calculations while this effect has to be carefully simulated for the final implementation of the design. The differential inductance of the resonator is $[\mathbf{1}]$ :

$$
L_{\text {perlen }}=\left(\frac{\mu_{0}}{\pi}\right) \log \left\{\frac{\pi \cdot s}{W+t_{c}}+1\right\}
$$

where $s$ is the gap between the conductors, $W$ is the conductor width and $t_{c}$ is the conductor thickness.

From (3.1), for our particular NPN transistor size of $6 \mu \mathrm{m}$ and for zero bias varactor, the per-segment capacitance is $C_{\text {seg }}=250 \mathrm{fF}$.

Knowing the width, spacing and thickness of the resonator as explained earlier in this chapter and using (3.2), the per-unit-length differential inductance of the line is $0.53 \mu \mathrm{H} / \mathrm{m}$. 
The per-unit-length differential capacitance of the resonator can now be calculated based on the value of the desired $Z_{0}$. We have designed our resonator with a relatively high $Z_{0}$ of $70 \Omega$. Substituting $C_{s e g}, L_{\text {perlen }}$ and $C_{0}$ values into (2.19), the oscillation frequency would be $17.45 \mathrm{GHz}$.

The most significant power loss mechanism for an RTWO is $I^{2} R$ power dissipated in the interconnect instead of $C V^{2} f[1]$ and it is determined based on (3.3):

$$
P_{d i s}=\frac{V_{C C}{ }^{2}}{Z_{0}{ }^{2}} R_{\text {loop }}
$$

where $Z_{0}$ is the characteristic impedance of the line and $R_{\text {loop }}$ is the total resistance of the resonator.

Once the oscillation starts in the travelling-wave mode in an RTWO, it takes little power to sustain it because the energy goes into charging and discharging the base capacitance becomes transmission line energy and this offers potential power savings as losses are not related to $C V^{2} f$ [1]. Otherwise for just driving the available base capacitance of each transistor, $242 \mathrm{fF}$, at this frequency, more current would be required from the available supply if the loss mechanism were $C V^{2} f$.

From (2.24), the resonator's quality factor of an RTWO is depending on parallel resistance and differential inductance of the resonator. This means that as we increase the width of the resonator, microstrip in our case, $R_{p}$ increases while $L$ decreases and this leads to a higher value of $Q$. 
However, as we increase the width of the line for microstrip structures, we decrease the value of the $Z_{0}$ and hence increasing the power dissipation of the resonator based on (3.3). Furthermore, low values of $Z_{0}$ can cause oscillation start-up difficulty.

For our design, in order to have low power consumption and also relatively small resonator geometry, a $Z_{0}$ of $70 \Omega$ has been chosen for the microstrip structure. A lower value of $Z_{0}$ for the same number of gain stages can improve the phase noise while higher $Z_{0}$ is beneficial for:

1 Reducing the power dissipation of the resonator based on (3.3)

2 Making the oscillation start-up easier as the start-up condition for an RTWO is, [38]:

$$
\left(\frac{Z_{\text {loaded }, 0}}{2} g_{m}\right) e^{-\alpha l} \geq 1 \rightarrow g_{m} \geq \frac{2}{Z_{\text {loaded }, 0}} e^{\alpha l}
$$

where $Z_{\text {loaded, }, 0} / 2$ represents the effective loading impedance of the gain stages and $\alpha$ is the attenuation of the resonator.

The start up condition of an RTWO can be calculated based on (2.11) as the open loop gain of an RTWO is similar to the gain of a distributed amplifier.

3 The higher the characteristic impedance, the higher inductance-per-unit length for CPS structure and the smaller the size of the resonator

We can conclude that a compromise between electrical parameters and the size of the resonator is required to meet some requirements specific to an application. 


\subsubsection{Phase Noise Analysis}

As explained in Chapter 2, [19] contains a detailed study on the phase noise analysis of CMOS RTWOs. This reference analyzes the RTWO based on a superposition of multiple $\lambda / 2$ SWOs and provides an equation for the phase noise of an RTWO utilizing CMOS $-g_{m}$ gain cells.

This reference has broken the circuit's noise sources into

1) resonator noise source,

2) differential pair noise source, and

3) tail current noise source.

We are using the same approach in order to analyze and calculate the phase noise of our bipolar RTWO.

As shown in Figure 3.3, we have biased our gain stages by means of two large resistors at the collector of the transistors while the collector and base terminals are tied together. These two resistors are used instead of the PMOS devices in [19] and they can contribute to the phase noise of the circuit by lowering the $R_{p}$ and hence the $Q$ of the resonator.

In order to estimate the resonator's phase noise contribution, the first step is to calculate the $Q$ of the line. (2.24) shows how the quality factor of a distributed resonator can be calculated based on the parallel resistance and differential inductance of the 
resonator. However extracting the parallel resistance of a transmission line is not very convenient using EM simulations.

Instead, we have used (3.5) to calculate the unloaded quality factor of the resonator:

$$
Q_{\text {unloaded }}=\frac{1}{\frac{R_{S}}{L \omega_{0}}+\frac{G}{C \omega_{0}}}
$$

where $R_{S}$ is series resistance of the line, $G$ is the shunt conductance of the line, $L$ and $C$ are the per-unit-length inductance and capacitance of the line.

The value of the lumped components are extracted from EM simulations and based on (3.6) [39]:

$$
\begin{aligned}
& R=\operatorname{real}\left(\gamma Z_{0}\right) \\
& G=\operatorname{real}\left(\frac{\gamma}{Z_{0}}\right) \\
& L=\operatorname{imag}\left(\gamma Z_{0}\right) \\
& C=\operatorname{imag}\left(\frac{\gamma}{Z_{0}}\right)
\end{aligned}
$$

where $\gamma$ is the propagation constant and $Z_{0}$ is the characteristic impedance of the line.

Our Momentum EM simulation results show $Z_{0}=64.5 \Omega, R=1976.85 \Omega / \mathrm{m}$, $G=0.475 \widetilde{J} / \mathrm{m}, L=0.4234 \mu \mathrm{H} / \mathrm{m}$ and $C=102 \mathrm{pF} / \mathrm{m}$. Substituting these values into (3.5), the quality factor of our resonator at $18 \mathrm{GHz}$ would be 12.15 . This value of unloaded quality factor leads to $R_{p}=175.5 \Omega$, using (2.24). 
Figure 3.5 shows the equivalent circuit of our RTWO which is used to calculate the phase noise of the circuit.

Considering the effect of $1 \mathrm{~K} \Omega$ biasing resistors in Figure 3.5, the effective total parallel resistance of the unloaded resonator will be $161.3 \Omega$ based on (3.7), which corresponds to the $Q$ value of 11.17 .

$$
R_{p-e f f}=4 R_{\text {bias }}\left\|4 R_{\text {bias }}\right\| R_{p}
$$

where $R_{p-e f f}$ is the effective parallel resistance of the resonator.

According to Leeson's equation given in (2.22), the phase noise contribution of the resonator at $\omega_{m}$ offset from the carrier frequency is:

$$
L\left(\omega_{m}\right)_{\text {resonator }}=\frac{2 k T R_{p-e f f}}{V_{0}^{2}}\left(\frac{\omega_{0}}{2 \omega_{m} Q}\right)^{2}
$$

where $V_{0}$ is the differential output voltage swing.

The half coefficient in (3.8) compare to the phase noise contribution of a $\lambda / 2 \mathrm{SWO}$ and $L C$-tank oscillator is due to the coupling effect between the gain stages in an RTWO. However, the output voltage amplitude of an RTWO is half of SWO, shown in (2.7). Reference [40] verifies phase noise improvement of strongly injection locked quadrature $L C$-VCO by solving differential equations. 

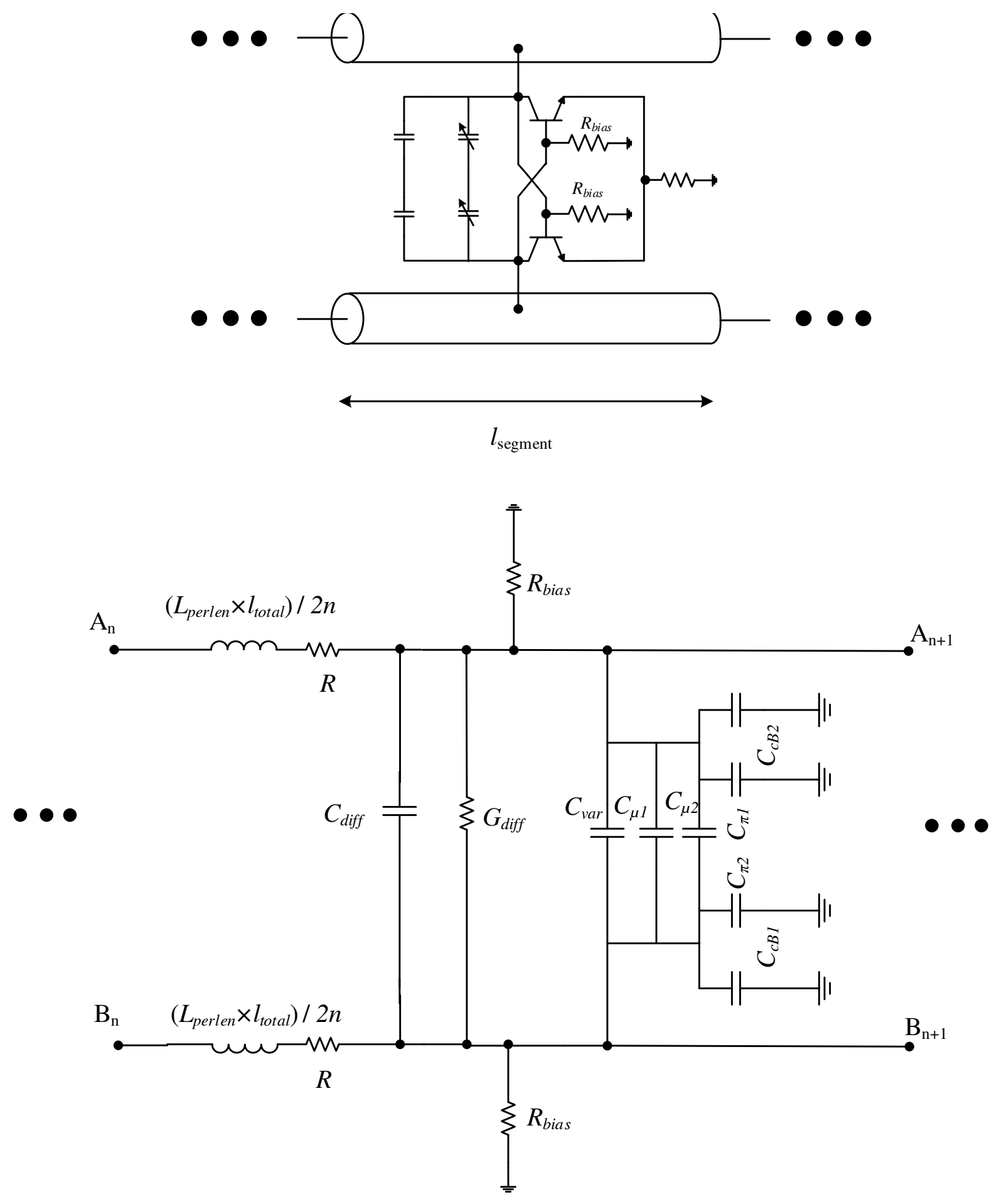

Figure 3.5 Equivalent circuit of our RTW-VCO used to calculate the phase noise 
Noise generated by the differential pairs using bipolar transistors can arise from collector shot noise, base resistance thermal noise and base shot noise [41]. In our theoretical calculations we have ignored the effect of base shot noise for simplicity and we have assumed that collector shot noise and base thermal noise are the main noise contributors.

Calculating the phase noise contribution of the differential pair is not as straightforward as the resonator phase noise since the noise is transferring to the output only at zero-crossing and when both transistors are ON. This is due to the fact that the OFF transistor obviously contributes no noise, and the $\mathrm{ON}$ transistor behaves like an amplifier with a fixed and constant value of tail current and $G_{m}$ would be zero during this time.

The noise from the differential pair is sampled by the finite width window due to the switching action and the power spectral density of this sampled noise is white. This window samples the noise of $V_{n}$ at the base of the transistor at every zero-crossing where both transistors are $\mathrm{ON}[\mathbf{4 2}]$.

This effect has been shown in Figure 3.6. The term $T_{s}$ is the time drift which advances or retards the time of zero-crossing and $S$ is the slope of the voltage at the switching time.

The value of $T_{s}$ can be calculated from the well-known $I-V$ characteristic of a switching pair shown in Figure 3.7. In a well-designed switching pair, the output amplitude should be much larger than $\Delta v$ to completely turn $\mathrm{ON}$ one branch and turn the other branch OFF. As a result $\Delta v$, shown in Figure 3.7, defines the linear region of a 
differential pair where both transistors are ON. This value for bipolar transistor is approximated by $4 V_{T}$, where $V_{T}$ is the thermal voltage.

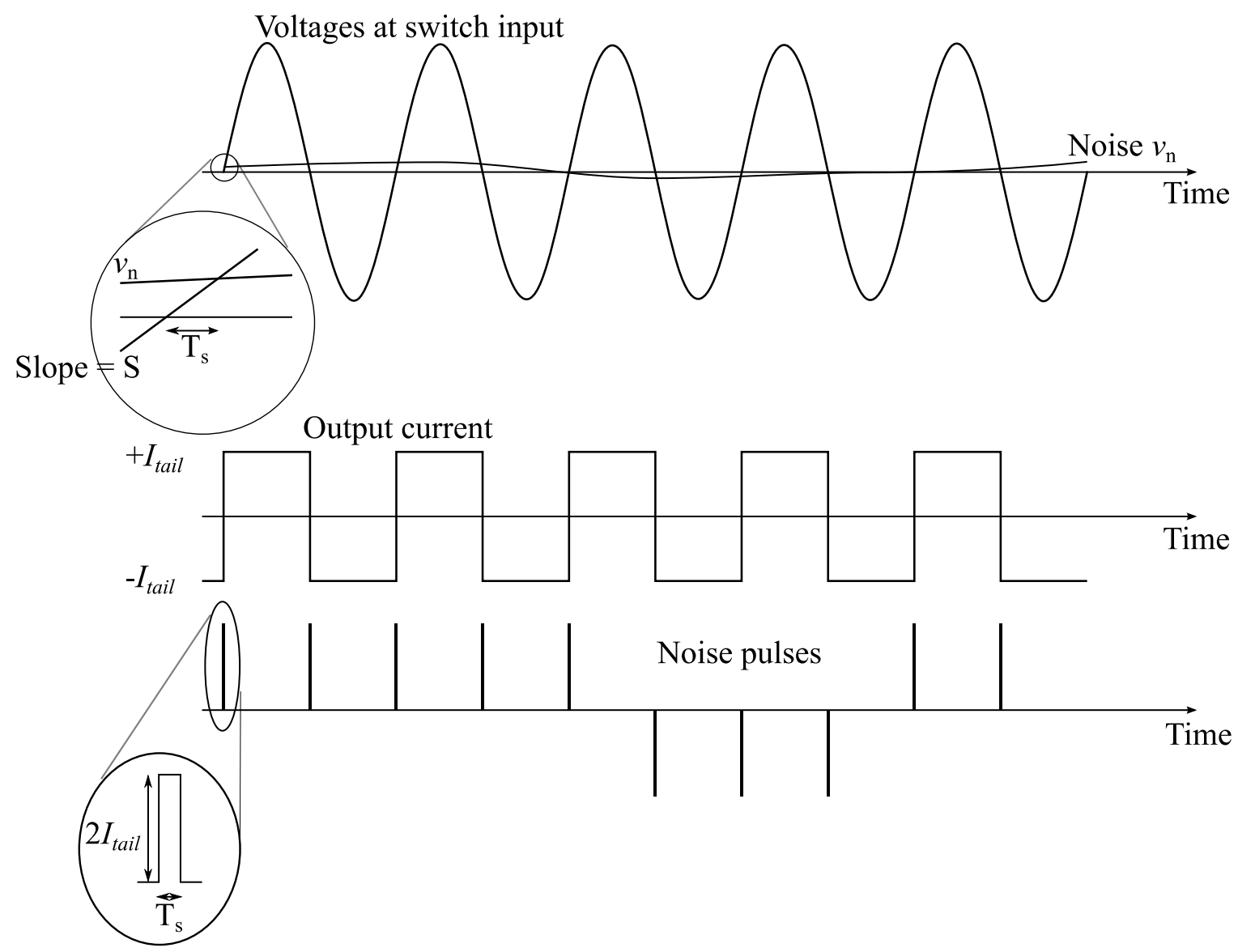

Figure 3.6 Differential pair input voltage and output current showing the noise model

From Figure 3.7 and considering the sampling action of the noise, if we can find the width of the sampling window or $T_{s}$, which is the time representative of $\Delta v$, then we can simply multiply the power spectral density of the transistor's current noise by this window and average it for every twice of the oscillation frequency since there are two zero-crossing over each cycle. 

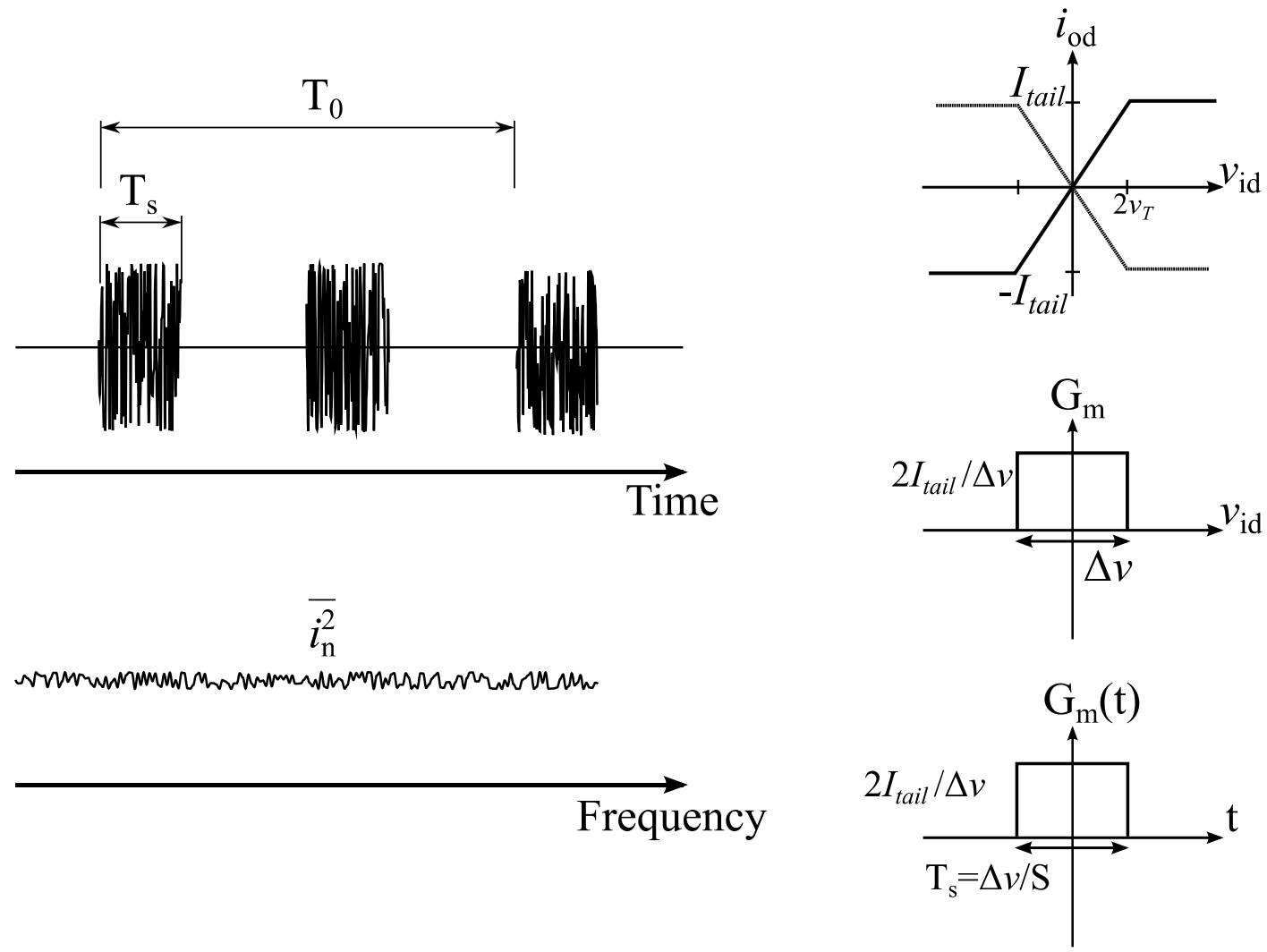

Figure 3.7 Switching pair $I-V$ curve and transconductance of the switching pair

In order to calculate the value of $T_{s}$, it is important to realize that the switches contribute noise to the output when they are both $\mathrm{ON}$ which is only during a window of $\Delta v$ width. For a sine-wave differential output voltage of $V_{i d}=2 A \sin \left(\omega_{0} t\right)$, since the value of $\Delta v$ should be much smaller than the amplitude of the voltage swing which is $2 \mathrm{~A}$ in this case, the value of $\sin \left(\omega_{0} t\right)$ in $V_{i d}$ expression should be very small. As a result, using a Taylor's series expansion of a sine function, $\sin \left(\omega_{0} t\right)$ can be approximated by $\omega_{0} t$

From the above analysis, the value of $\Delta v$ and $T_{s}$ can be defined based on (3.9): 


$$
\begin{gathered}
\Delta v=2 A \omega_{0} T_{s} \\
T_{s}=\frac{\Delta v}{2 A \omega_{0}}=\frac{\Delta v}{S}
\end{gathered}
$$

where $S=2 A \omega_{0}$ is the slope of sine-wave at zero-crossing.

As a result, the sampling window width for the noise contribution of the differential pair is $T_{S}=\Delta v / S$.

Finally the power spectral density of the output current noise can be defined based on (3.10):

$$
\overline{l_{o, n}^{2}}=\overline{l_{l, n}^{2}} \times \frac{2 T_{S}}{T_{0}}
$$

The large signal transconductance of the differential pair, $G_{m}$ is the derivative of the $I$ $V$ characteristic, as shown in Figure 3.7, and it is $2 I_{\text {tail }} / \Delta v$.

Applying (3.10) to our NPN differential pair, the noise power spectral density of the base thermal noise and collector shot noise after being sampled are:

$$
\begin{aligned}
& \overline{V_{l, n}{ }^{2}}=\left(4 K \operatorname{Tr}_{B}+\frac{2 K T}{G_{m}}\right) \\
& \overline{l_{o, n}{ }^{2}}=\overline{{V_{b n}}^{2}} G_{m}{ }^{2}+\overline{l_{c n}{ }^{2}}
\end{aligned}
$$




$$
\begin{aligned}
\overline{l_{o, n}^{2}}=\overline{l_{\text {bn,o }}{ }^{2}} & +\overline{l_{c n, o}{ }^{2}}=\left(4 K \operatorname{Tr}_{B} G_{m}{ }^{2}+2 K T G_{m}\right) \frac{2 T_{s}}{T_{0}} \\
& =4 K T r_{B} \times\left(\frac{2 I_{\text {tail }}}{\Delta v}\right)^{2} \times \frac{2 \Delta v}{S T_{0}}+2 K T \times \frac{2 I_{\text {tail }}}{\Delta v} \times \frac{2 \Delta v}{S T_{0}} \\
& =\frac{4 K T r_{B} I_{\text {tail }}{ }^{2} \omega_{0}}{V_{T} \pi S}+4 K T I_{\text {tail }} \frac{\omega_{0}}{\pi S}
\end{aligned}
$$

Following the Leeson's phase noise equation and extracting the fitting factor of $F$ for bipolar transistors, the phase noise contribution of a bipolar differential pair can be calculated as follows:

$$
\begin{aligned}
& \mathcal{L}\left(\omega_{m}\right)_{\text {diff_pair }}=\frac{2 F k T R_{p-e f f}}{V_{0}{ }^{2}}\left(\frac{\omega_{0}}{2 \omega_{m} Q}\right)^{2} \\
& F=\frac{r_{B} I_{\text {tail }}^{2} R_{p-e f f} \omega_{0}}{V_{T} \pi S}+\frac{R_{p-e f f} I_{\text {tail }} \omega_{0}}{\pi S}
\end{aligned}
$$

From the well-known harmonic amplitudes for the square wave, the voltage waveform $V(t)$ across the resonator is [19]:

$$
V(t)=V_{0} \sum_{k=0}^{n_{h}} \frac{1}{2 k+1} \sin \left\{(2 k+1) \omega_{0} t\right\}
$$

where $n_{h}$ is the number of harmonics which are sustained in a distributed resonator as explained in (2.20).

From (3.13), the slope of the voltage waveform at zero crossing, $S$, can be defined based on (3.14).

$$
S=\left.\frac{d V(t)}{d t}\right|_{t=0}=\omega_{0} V_{0}\left(n_{h}+1\right)
$$


Substituting (3.14) into (3.12) and knowing the output voltage amplitude of an RTWO, the $F$ in our case can be defined based on (3.15).

$$
\begin{gathered}
V_{0}=\frac{2 I_{\text {tail }} R_{p-e f f}}{\pi} \\
F=\left(\frac{r_{B} I_{\text {tail }}{ }^{2} R_{p-e f f}}{2 V_{T} \pi} \times \frac{1}{V_{0}\left(n_{h}+1\right)}+\frac{R_{p-e f f} I_{\text {tail }}}{\pi} \times \frac{1}{V_{0}\left(n_{h}+1\right)}\right) \\
=\left(\frac{r_{B} I_{\text {tail }}}{V_{T}}+1\right) \times \frac{1}{2\left(n_{h}+1\right)}
\end{gathered}
$$

The first term in (3.15) is referring to the base thermal noise and second term is referring to the collector shot noise.

From (3.15) we can state that as we increase the number of gain cells in an RTWO, the noise factor gets smaller, independent of transistor characteristics, and hence the phase noise is improved. This surprising effect has been noticed in the literature by solving differential equations. However, we have also mentioned in (2.16) that as we increase the number of gain stages, the resonator cut-off frequency increases while the oscillation frequency is constant and more harmonics can be sustained in the resonator. This effect has been proven for strongly injection locked quadrature $L C$-VCO in [40].

Finally the total phase noise of the RTWO using bipolar differential pair is:

$$
\begin{gathered}
\mathcal{L}\left(\omega_{m}\right)_{R T W O}=\frac{2 F k T R_{p-e f f}}{V_{0}{ }^{2}}\left(\frac{\omega_{0}}{2 \omega_{m} Q}\right)^{2} \\
F=1+\left(\frac{r_{B} I_{\text {tail }}}{2 V_{T}}+\frac{1}{2}\right) \frac{1}{\left(n_{h}+1\right)}
\end{gathered}
$$


The first term in (3.16) is the noise factor contribution of the resonator, the second term is the noise factor contribution of the base thermal noise and the last term is showing the effect of collector shot noise on the phase noise performance of the RTWO.

(3.16) also suggests that the phase noise contribution of both base thermal noise and collector shot noise are independent of transistor size and switching speed. This is due to the fact that as the switching speed decreases or the sampling window gets wider, the value of $G_{m}$ which is the slope of the $I-V$ curve in Figure 3.7 is also getting smaller and these two factors cancel, seen in (3.11). As a result, the noise factor referring to the collector shot noise is a constant value of 0.5 .

The last source of noise in our RTWO is the tail current noise source. For simplicity, we have ignored the noise contribution of this tail resistor in our calculations since the effect of this noise source can be minimized by using a bypass capacitor.

From (2.20), $n_{h}$ is 0 in our case because we have used four gain stages in our design and our output voltage is a sine-wave with a finite slope. Substituting our calculated values of $Q$ and $R_{p-e f f}$ for the bias point of $I_{C}=2.85 \mathrm{~mA}$, the total calculated phase noise of the RTWO is $-111.6 \mathrm{dBc} / \mathrm{Hz}$ at a $1 \mathrm{MHz}$ offset from the $18 \mathrm{GHz}$ carrier frequency, ignoring the effect of tail current noise.

Comparing (3.16) to the phase noise equation derived in [19] for CMOS-based RTWOs, which is shown in (2.24), we can state that the phase noise of a bipolar RTWO for the same value of output voltage is better than the phase noise of a CMOS-based RTWO, if collector shot noise is the main contributor of the noise. 
This is due to the fact that the noise factor, $F$, in bipolar transistors can be smaller than in CMOS devices depending on the value of the channel noise coefficient of a MOS transistor, $\gamma$. The value of $\gamma$ depends on the carrier concentration in the channel, current density and the device geometry and it is a value between 0.67 for long channel MOSFETs and one for shorter channel devices [43]. Assuming the highest value of 2 for the noise factor of short-channel CMOS RTWO, due to $1+\gamma$, we can state that if the noise factor contribution of the base thermal noise is lower than 0.5 , then the bipolar switching pair contributes less thermal noise than that of CMOS pair. It is also known that the flicker noise corner of bipolar transistors is happening at lower frequency than CMOS transistors and they have better flicker noise performance as well.

However, bipolar VCOs are different than CMOS VCOs because we have to take care of the output swing not to forward bias the collector-base junction.

Our calculated results are compatible with [44], while it is less complex and we have re-arranged our calculations based on the well-known Leeson's equation for the phase noise. Furthermore, Reference [44] has done a phase noise analysis for a bipolar $L C$ VCO and our calculations shows the phase noise performance of a bipolar RTWO for the first time.

\subsection{Post-Layout Simulation Results}

In order to have accurate simulation results, a full EM simulation of the resonator in combination with electrical simulation of the rest of the circuit was unavoidable for every stage of the design. All of the presented simulation results are obtained from the 
combination of the s-parameter file of the resonator, analyzed in Momentum, and the $R L C$ extraction of the gain cells being laid out in Cadence. The main characteristics of the technology we have used in this design, $0.25 \mu \mathrm{m}$ BiCMOS, are summarised in Table 2.

Table 2 Main characteristics of Qubic4XI 0.25 $\mu \mathrm{m}$ BiCMOS process from NXP Semiconductors

\begin{tabular}{|c|c|c|c|}
\hline$f_{\boldsymbol{T}} / \boldsymbol{f m a x}$ & Substrate resistivity & $\boldsymbol{\varepsilon}_{\boldsymbol{r}}$ & Top metal thickness \\
\hline $200 \mathrm{GHz} / 180 \mathrm{GHz}$ & $200 \Omega \mathrm{m}$ & 11 & $4 \mu \mathrm{m}$ \\
\hline
\end{tabular}

Figure 3.8 shows the simulated output spectrum of the RTWO. The supply voltage is simulated at $4.5 \mathrm{~V}$ since there are $1 \mathrm{~K} \Omega$ resistors used to bias the collector of the device.

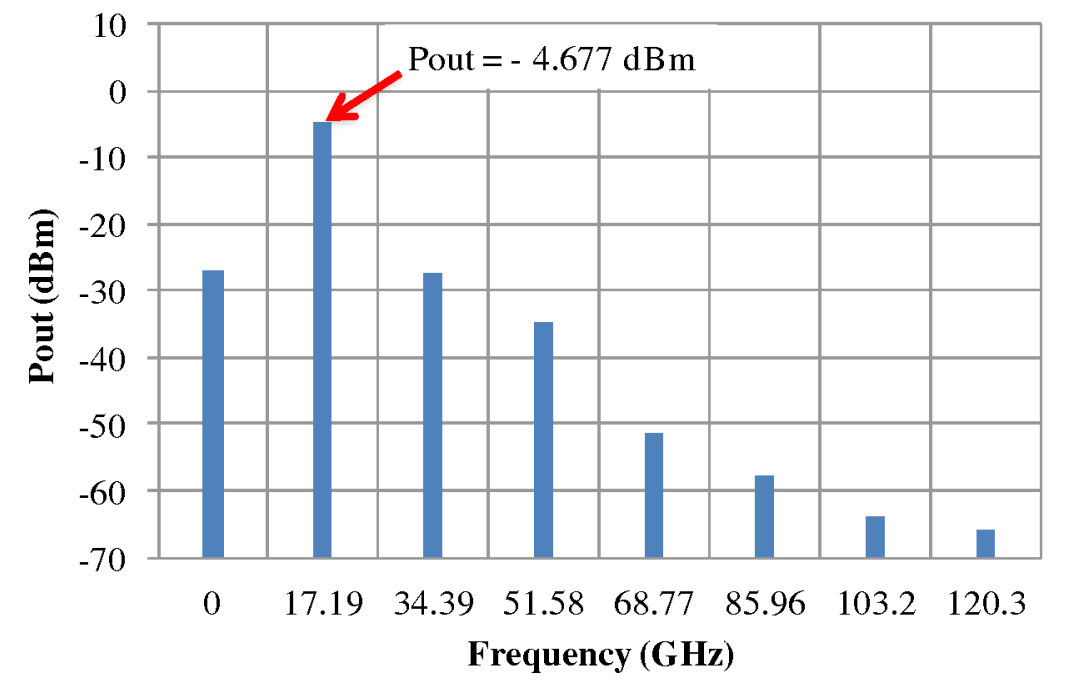

Figure 3.8 Simulated output spectrum of the RTWO showing the fundamental tone

As seen from this figure the oscillation frequency of the RTWO is $17.19 \mathrm{GHz}$ for a tuning voltage of $0 \mathrm{~V}$ and the second harmonic is $22.8 \mathrm{~dB}$ smaller than the fundamental tone. From the same figure, the simulated output power at the output nodes of the buffers 
is $-4.67 \mathrm{dBm}$. Both the oscillation frequency and the output power are in close agreement with our calculated results shown earlier in this chapter.

Figure 3.9 shows the simulated phase noise of our RTWO at a $1 \mathrm{MHz}$ offset from the carrier frequency. From this figure, the phase noise of the RTWO is $-106.8 \mathrm{dBc} / \mathrm{Hz}$.

This number is $4.8 \mathrm{~dB}$ higher than our calculated value. This can be due to the fact that in our calculations we have assumed that the output voltage is a perfect sine-wave and the slope of it at the zero crossing is $\omega_{0} V_{0}$, based on (3.14). However, in reality the shape of output current in our circuit is not a perfect square wave, and the slope of the voltage at zero-crossing is lower than our assumed value. The other reason can be the neglect of tail resistor phase noise and the simplification of the noise contribution of a bipolar transistor in the analytical calculation. Furthermore, $R L C$ extraction of the gain cells can add parasitic resistance to the resonator and lower the quality factor of the resonator.

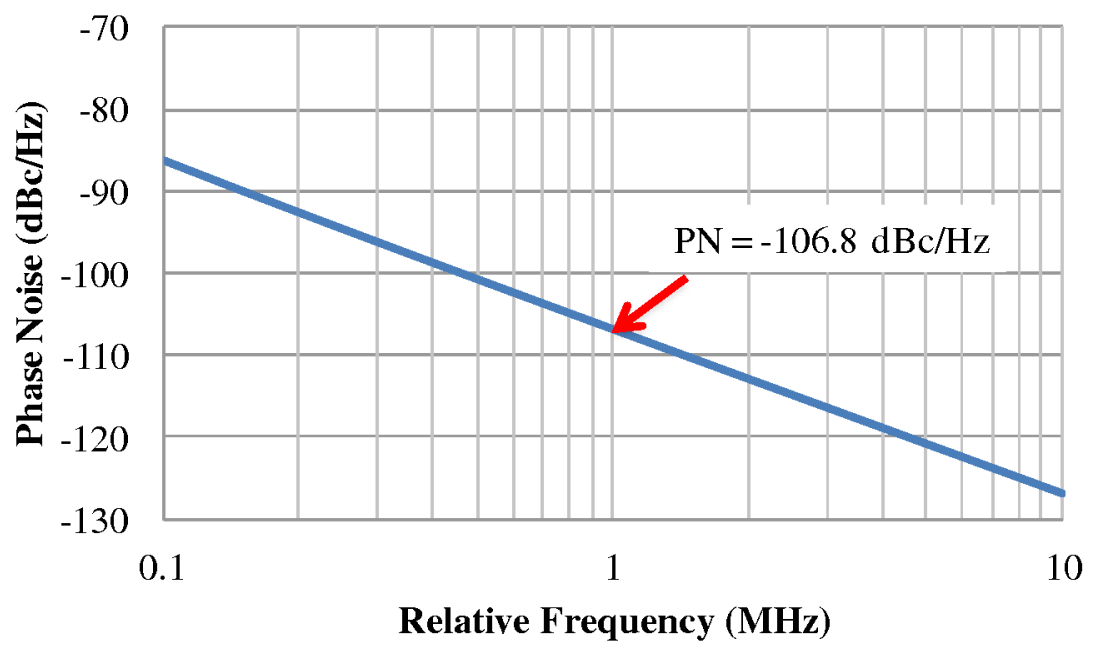

Figure 3.9 Simulated phase noise at a $1 \mathrm{MHz}$ offset from the carrier frequency 
Figure 3.10 shows the transient response of our circuit. This figure also illustrates the symmetry of our resonator. From this figure the maximum phase error between each two segments is $2.5^{\circ}$. This shows that our resonator is quite symmetrical while it is simple and small. The total length of our resonator, $1.488 \mathrm{~mm}$, is smaller than other published RTWOs in the same range of frequency since we have used simple microstrip lines with orthogonal structure for the implementation of the cross-overs and high characteristic impedance which leads to smaller line widths.

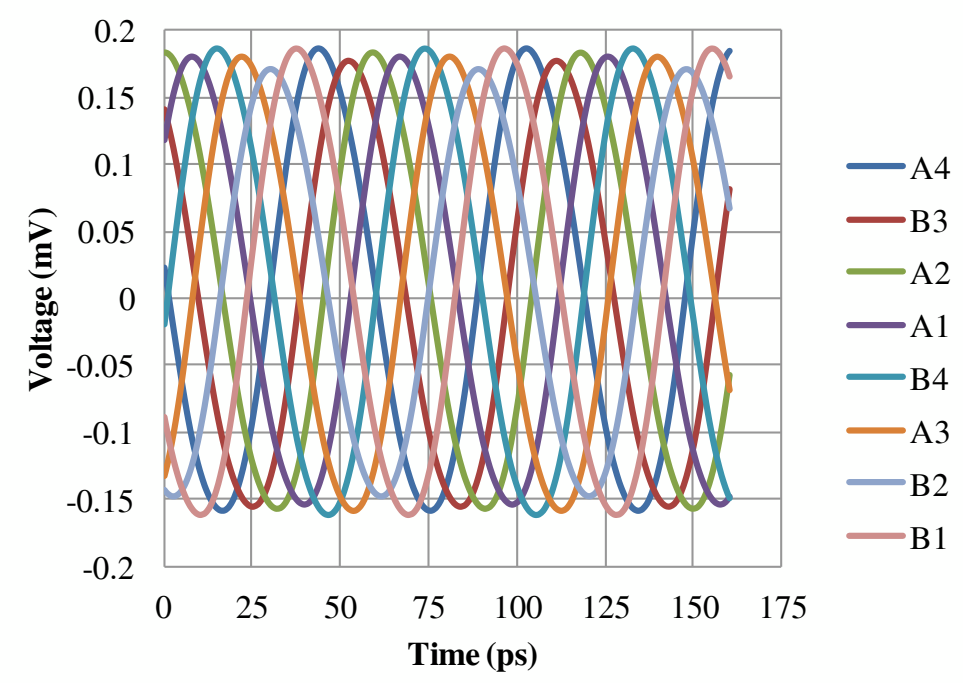

Figure 3.10 Simulated transient response of the RTWO

\subsection{Characterization of the RTWO}

The measurement was performed on wafer using high frequency 8-pin probes. The DC bias voltages have been connected to the circuit through the power tips of the high frequency eight-pin probes. A high frequency HP8564E spectrum analyzer is used to measure the oscillation frequency, output power and the phase noise. The measured 
insertion loss from the probes to the spectrum analyzer is $3 \mathrm{~dB}$ at $17 \mathrm{GHz}$. Therefore the measured output power should be adjusted for this loss.

Figure 3.11 shows the die photomicrograph of the RTWO realized in a $0.25 \mu \mathrm{m}$ BiCMOS process from NXP Semiconductors. The RTWO's total size, excluding the pads, is $0.57 \mathrm{~mm}^{2}$.

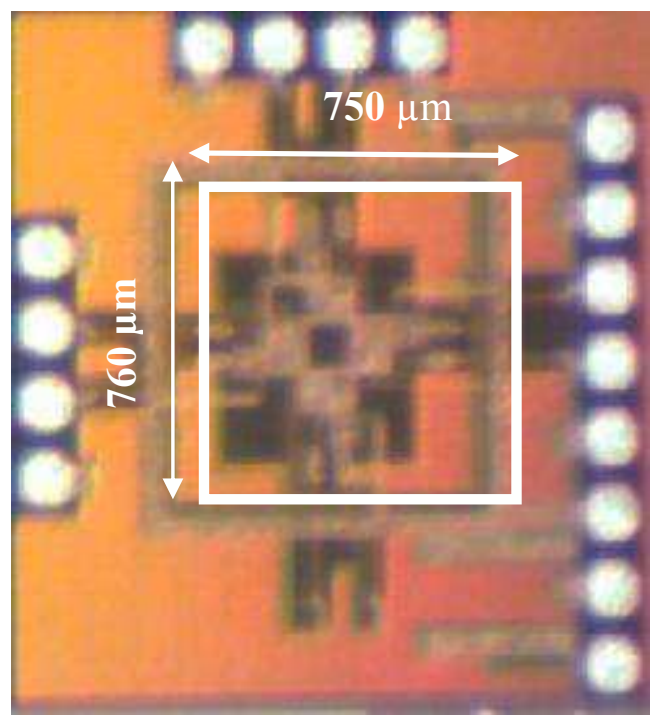

Figure 3.11 Die photomicrograph of the RTWO fabricated in NXP $0.25 \mu \mathrm{m}$ BiCMOS process

Figure 3.12 shows the spectrum of the RTWO's output. From this figure, the measured center frequency of the RTWO is $17 \mathrm{GHz}$ with an output power of $-6.5 \mathrm{dBm}$ into $50 \Omega$. The second harmonic is $30 \mathrm{~dB}$ lower than the fundamental tone. Varying the tuning voltage from $0 \mathrm{~V}$ to $2.5 \mathrm{~V}$, the center frequency moves to $18.2 \mathrm{GHz}$.

The measured phase noise of the RTWO, as shown in Figure 3.13, is $-104.2 \mathrm{dBc} / \mathrm{Hz}$ at a $1 \mathrm{MHz}$ offset from the $17 \mathrm{GHz}$ centre frequency. Since our RTWO is a free-running 
oscillator it is not possible to have a very accurate phase noise measurement and we have captured both better and worse results for the phase noise of the RTWO.

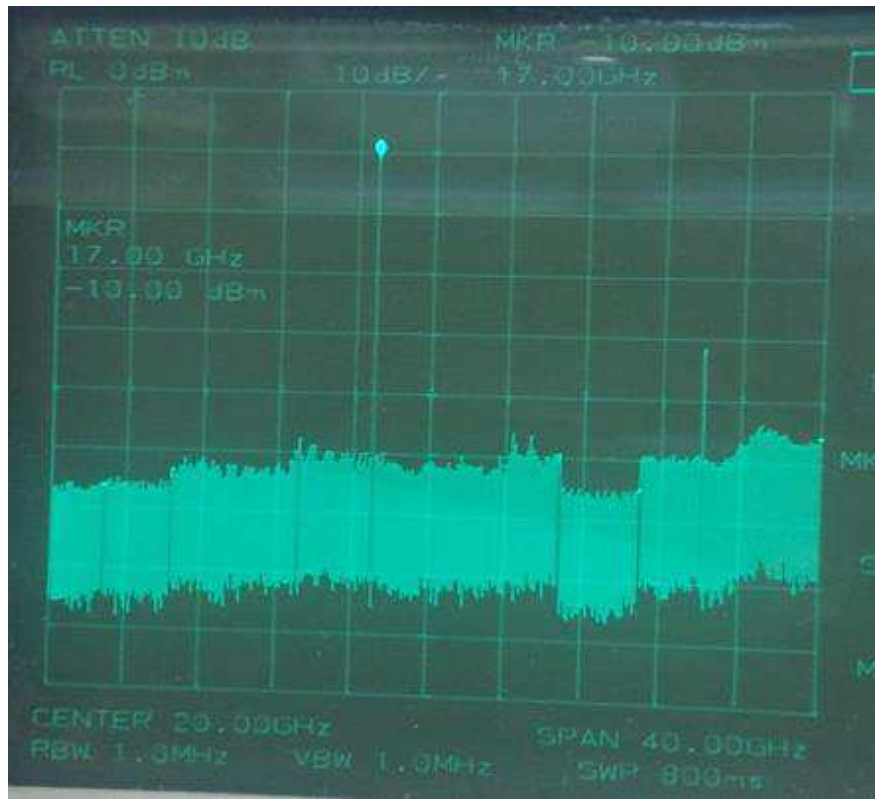

Figure 3.12 Measured output spectrum of the RTWO showing the fundamental at $17 \mathrm{GHz}$

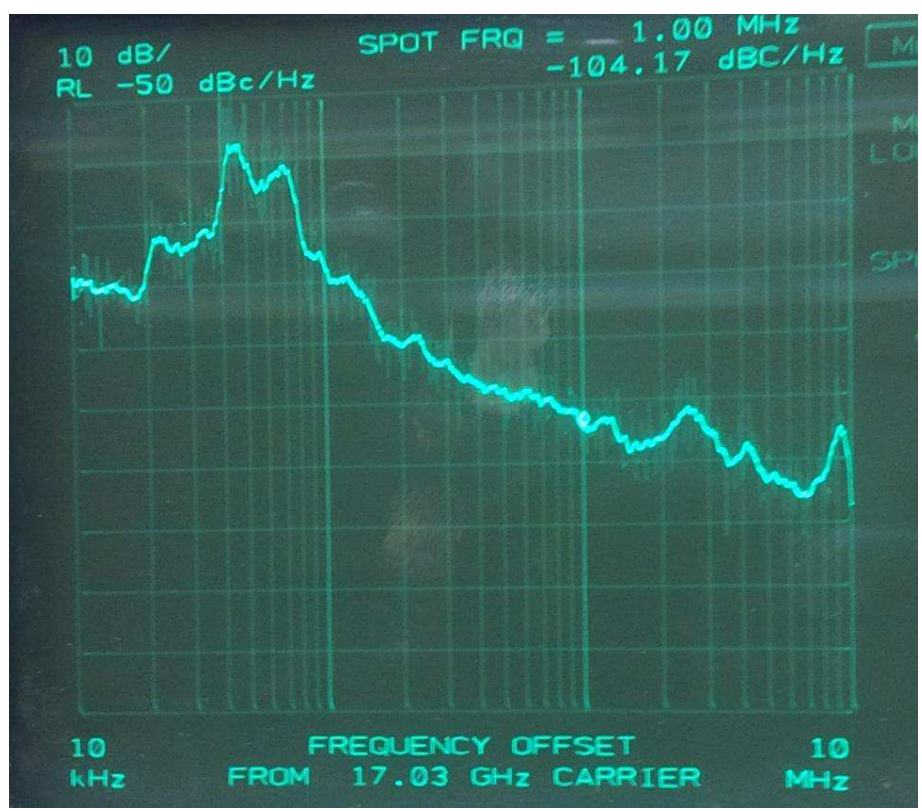

Figure 3.13 Measured phase noise at a $1 \mathrm{MHz}$ offset from the $17 \mathrm{GHz}$ centre frequency 61 
In order to measure the phase shift between the segments we have proposed an untried frequency domain method which will be discussed in chapter 4 .

\subsection{Summary}

In this chapter, the analytical expressions, design methodology, simulation and measurement results of a $17.5 \mathrm{GHz}$ RTWO have been presented.

We have also derived an equation for the phase noise and noise factor of bipolar RTWOs in this chapter. Based on our calculations, the noise factor referring to the collector shot noise is independent of the device geometry, switching speed and bias point and it has a constant value of 0.5 in the current limiting region. The base shot noise factor on the other hand depends on the bias point and base resistance. In order to improve this factor for phase noise improvement, the value of $r_{B}$ has to be reduced and this can be done by using multiple fingers in the layout.

Comparing bipolar and CMOS switching pairs, we can state that if the collector shot noise is the main noise contributor, then a bipolar switching pair contributes less thermal noise than a CMOS switching pair because the channel noise coefficient of CMOS pairs, $\gamma$, is higher in value than 0.5 . Taking into account the effect of base thermal noise the exact value of $\gamma$ is needed, in order to compare the two while $\gamma$ is a number between 0.67 and 1 and it is depending on current density, carries concentration and channel length.

The presented measurement results of the RTW-VCO shows a tuning range of $6.3 \%$ and phase noise of $-104.2 \mathrm{dBc} / \mathrm{Hz}$ at a $1 \mathrm{MHz}$ offset from the $17 \mathrm{GHz}$ carrier frequency. 
The measurement results of the circuit are in close agreement with our analytical calculated results and also extracted simulation results thanks to EM co-simulations.

Table 3 shows a summary of the RTWO performance and compares it to previously published RTWOs.

From this table, we can see that the figure-of-merit (FOM) of our design is comparable with other published designs while our design gives access to eight output phases with $45^{\circ}$ of phase step and this is essential in some applications like in phasedarray transmitters where high resolution of beam-forming is required.

Table 3 Performance Summary of our RTWO compare to other recently published designs

\begin{tabular}{|c|c|c|c|c|c|c|c|}
\hline & $\begin{array}{c}\text { Simulated } \\
\text { results of } \\
\text { the RTWO }\end{array}$ & $\begin{array}{c}\text { Measured } \\
\text { results of } \\
\text { the RTWO }\end{array}$ & {$[\mathbf{3 7}]$} & {$[\mathbf{3 9}]$} & {$[\mathbf{4 5}]$} & \multicolumn{2}{|c|}{$[\mathbf{3 8}]$} \\
\hline Tech. & $\begin{array}{c}\mathbf{0 . 2 5} \boldsymbol{\mu m} \\
\text { BiCMOS }\end{array}$ & $\begin{array}{c}\mathbf{0 . 2 5} \boldsymbol{\mu m} \\
\text { BiCMOS }\end{array}$ & $\begin{array}{c}0.25 \mu \mathrm{m} \\
\text { BiCMOS }\end{array}$ & $\begin{array}{c}90 \mathrm{~nm} \\
\text { CMOS }\end{array}$ & $\begin{array}{l}0.18 \mu \mathrm{m} \\
\text { CMOS }\end{array}$ & $0.18 \mu \mathrm{m}$ CMOS \\
\hline $\begin{array}{c}\text { Freq. } \\
\text { (GHz) }\end{array}$ & $\mathbf{1 7 . 7 5}$ & $\mathbf{1 7 . 5}$ & 18 & 14 & 32 & 15 & 30 \\
\hline $\begin{array}{c}\text { Tuning } \\
\text { Range }\end{array}$ & $\mathbf{6 . 4 \%}$ & $\mathbf{6 . 3 \%}$ & $4 \%$ & $14 \%$ & $2.6 \%$ & $1.6 \%$ & $1.6 \%$ \\
\hline $\begin{array}{c}\text { DC Power } \\
\text { (mW) }\end{array}$ & $\mathbf{9 0}$ & $\mathbf{9 0}$ & 54 & 12 & 54 & 52 & 52 \\
\hline $\begin{array}{c}\text { Phase Noise } \\
\text { (dBc/Hz) }\end{array}$ & $\mathbf{- 1 0 7}$ & $\mathbf{- 1 0 4}$ & -98 & -109 & -108 & -112 & -104 \\
\hline $\mathbf{P}_{\text {out }}$ (dBm) & $\mathbf{- 4 . 5}$ & $\mathbf{- 6 . 5}$ & -5.5 & -11.3 & -9.1 & -8 & -15 \\
\hline FOMM $[39]$ & $\mathbf{- 1 6 8 . 5}$ & $\mathbf{- 1 6 5}$ & -158 & -182 & -169 & -163 & -161 \\
\hline $\begin{array}{c}\text { \# of Output } \\
\text { Phases }\end{array}$ & $\mathbf{8}$ & $\mathbf{8}$ & 8 & Half-quad & 8 & $\begin{array}{c}\text { Half- } \\
\text { quad }\end{array}$ & Quad \\
\hline
\end{tabular}

However, in order to improve the performance of this design, we could have added more dummy gain cells, for the same number of output phases and oscillation frequency, to improve the phase noise of the design. Improving the quality factor of the resonator 
was also possible by lowering the characteristic impedance of the microstrip structure we have employed in this design. However, this can lead to a bigger resonator.

As was explained at the beginning of the chapter, we have tied together the collectors and bases of our cross-coupled pairs and we have not used a DC blockage capacitor to bias the base and collector terminals individually. This means that our design can give a maximum output swing of $0.8 \mathrm{~V}$ since beyond this, the base-collector junction will be forward biased.

Biasing these terminals individually can improve the output power and hence the phase noise but this will make the size of the die bigger. Furthermore, the addition of extra resistors to divide the voltage between base and collector, in order to keep the die size almost constant, can add to the phase noise of the circuit. 


\section{Chapter 4 RTWO Phase Shift}

\section{Measurement}

In order to take advantage of the multiple output phases of an RTWO, the phase step between different outputs of this structure should be constant and accurate. However, phase errors can occur due to device mismatch and layout asymmetry. This can limit the practical usefulness of this multiphase structure.

In this chapter, an untried method of phase shift measurement of a high frequency Rotary Travelling-Wave Oscillator (RTWO) is presented. Lack of symmetry in such a structure can result in oscillation in an unwanted mode rather than the travelling-wave mode. In order to determine the mode of the signal and also measure the phase shift between the outputs, we have proposed a preliminary phase shift measurement technique in the frequency domain for a high frequency RTWO.

\subsection{Conventional Phase Shift Measurement Approaches}

Measuring the phase at high frequency is not an easy task. For example measuring $90^{\circ}$ of phase shift at $18 \mathrm{GHz}$ corresponds to measuring a time delay of $13.89 \mathrm{ps}$, since,

$$
\Delta \varphi=\frac{\Delta t}{T} \times 360^{\circ}
$$

where $\Delta t$ is the time delay and $T$ is the period of the signals. 
The most common and unambiguous method for off-chip phase shift measurement is to measure the time delay between two signals using a high frequency digital oscilloscope. However, high frequency, real time, multi-channel oscilloscopes are quite expensive devices and they are not available in every lab, including in Carleton University's lab!

One solution to this is to use off-chip mixers or dividers to mix the signals to lower frequencies as at lower frequencies less expensive oscilloscopes can be used. However, even at low frequencies every piece of equipment used in down-conversion should be phased-matched otherwise they can directly contribute to phase measurement error.

The other known off-chip phase measurement approach is to use a mixer as a phase detector. Doing this, the RF and LO terminals of the mixer should be fed with the two signals between which we are attempting to measure the phase shift. The IF output of the mixer is a DC component which contains phase shift information as below:

$$
\cos \left(\omega_{0} t+\varphi\right) \times \cos \left(\omega_{0} t\right)=\frac{1}{2} \cos \left(2 \omega_{0} t+\varphi\right)+\frac{1}{2} \cos \varphi
$$

From this equation, we obtain a sinusoid with frequency of $2 \omega_{0}$ and a DC term of $+\frac{1}{2} \cos \varphi$.

Figure 4.1 shows our simulated results using the RTWO and an approximate model for a diode-based mixer. However, while this works in simulation, real mixers require a higher drive level than was needed in simulation. The higher the LO and RF signals, the closer the shape of the IF signal to a cosine-wave. 


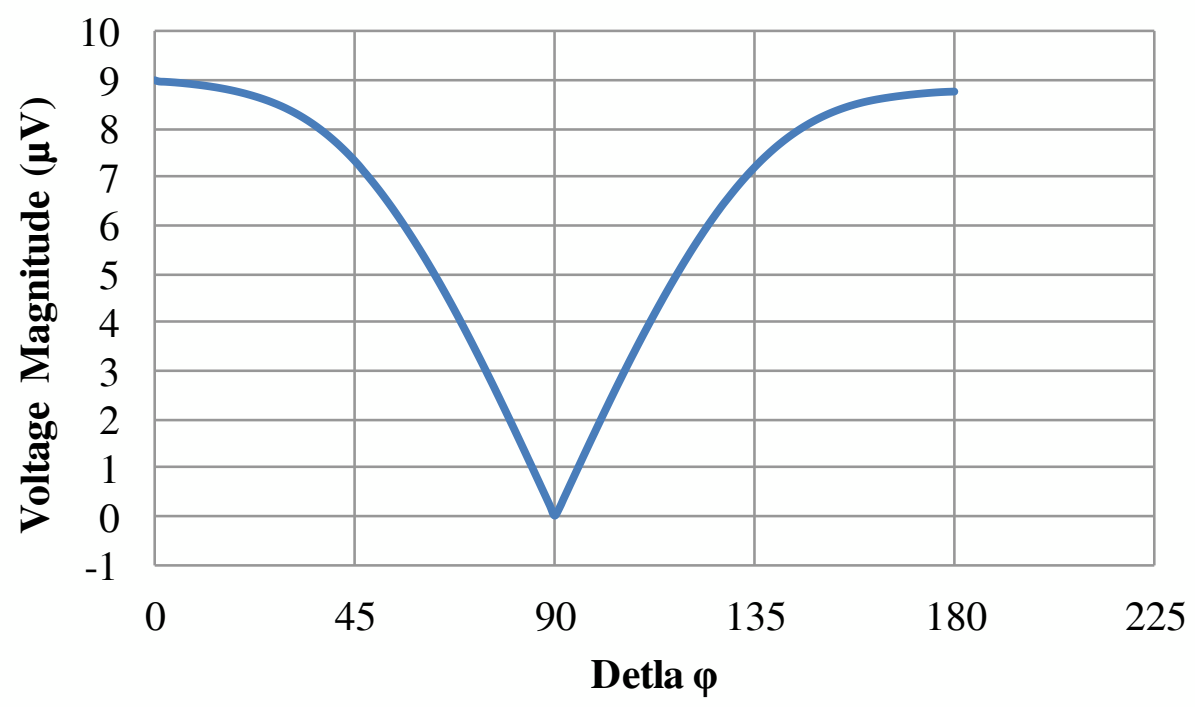

Figure 4.1 DC component of a diode-based mixer's IF output

From this figure, the phase shift between the segments can be determined based on the measured DC level. For example for $90^{\circ}$ of phase shift, the IF measured DC level should be zero.

However, the main problem of this off-chip approach is the need for high LO signals, close to $+13 \mathrm{dBm}$, in order to drive the mixer properly. In our case of $-9 \mathrm{dBm}$ of output power, considering the loss of the cables, the LO signal swing is not high enough to switch the mixer and this approach does not work for us in the lab. 


\subsection{Innovative Frequency Domain Phase Shift Measurement}

\section{Technique}

The RTWO can be analyzed as $N, \lambda / 2$, SWOs, shown in (2.7), with gain stages evenly distributed with time delay of $T_{0} /(2 N)$ and position offset of $\lambda /(2 N)$ across the ring, where $\lambda$ is wavelength. The superposing of $N$ stages of SWO can be calculated as [19]:

$$
V_{T W}(t, x)=\sum_{n=0}^{N-1}\left[2 A_{0} \cos \left\{\beta\left(x-\frac{n \lambda}{2 N}\right)\right\} \cos \left\{\omega_{0}\left(t-\frac{n T_{0}}{2 N}\right)\right\}\right]
$$

From (4.3), any mismatch in timing offset, e.g., due to the parasitic capacitance mismatch of the segments, or position offset, e.g., due to lack of symmetry in layout, can cause the signal to move in both clockwise and counter-clockwise directions. If the structure is fully symmetrical, the wave can only travel in one direction and all other terms cancel. Therefore, it is quite beneficial to determine if the wave is travelling at all before trying to find a way to get access to an expensive high frequency oscilloscope for more accurate phase shift measurement.

After laying out the RTWO with $N=4$ at $18 \mathrm{GHz}$, a Monte Carlo analysis is performed to characterize phase shift between nodes $A_{1}$ and $A_{3}$, shown in Figure 4.2, with process, temperature and power supply variation. Temperature and process variation had minimal effect. However, power supply variations resulted in unwanted mode of oscillation for some of the samples. 


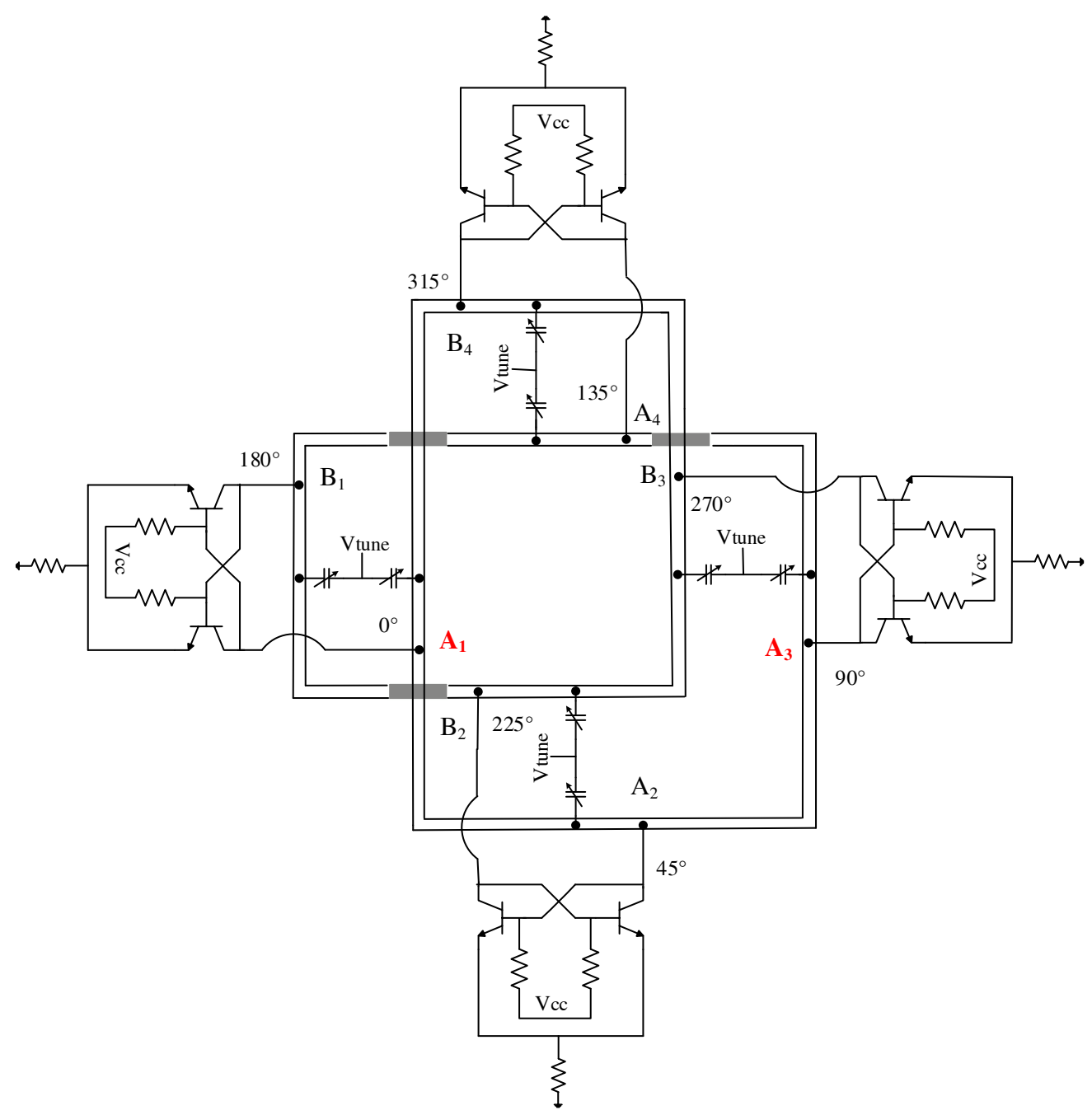

Figure 4.2 The RTWO schematic

Figure 4.3 and Figure 4.4 show the simulated time and frequency domain response for this undesired mode. The output spectrum is a single dominant fundamental tone while the time domain response of the circuit at $A_{1}$ and $A_{3}$, shows signals with different amplitudes but the same phase.

In our suggested frequency domain approach, in order to measure the phase shift, we have used an Agilent 11667A wideband DC-18 GHz power combiner to combine the 
outputs of the RTWO at different tapping points. Knowing the combined output power value, we do some vector analysis to convert the magnitude information of the combined signal into the phase difference.

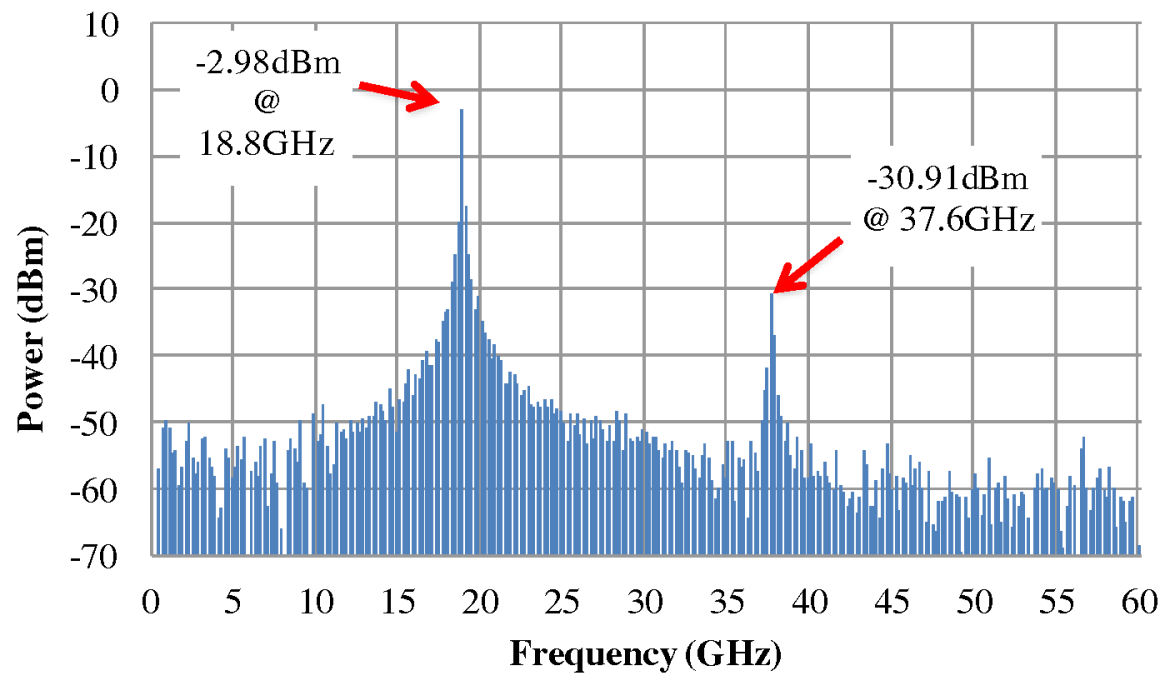

Figure 4.3 Simulated output spectrum under standing wave mode condition

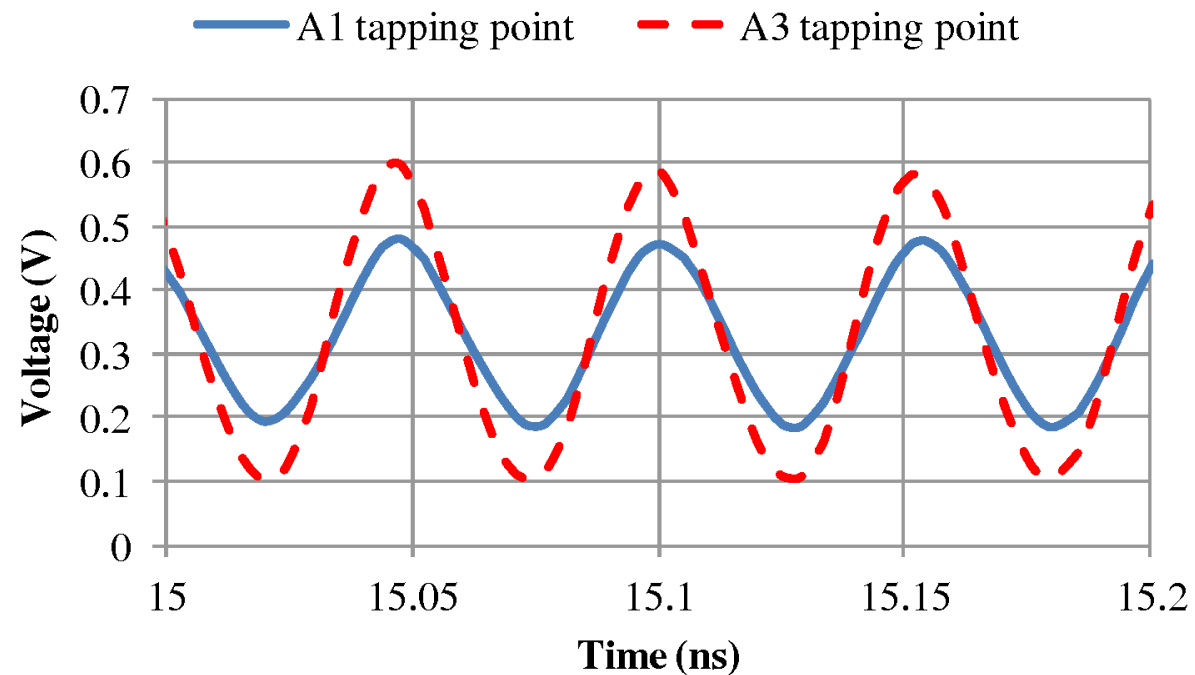

Figure 4.4 Simulated time domain voltage under standing wave mode condition 
In our circuit we have access to 4 different outputs with $90^{\circ}$ of phase shift between each pair. This means that in the ideal case where the two outputs have the same voltage magnitude of $V$, and the power combiner and all the cables are loss-less, the voltage magnitude of two outputs combined together, should be equal to $\sqrt{2} \times V$ if there is exactly $90^{\circ}$ of phase shift between them.

The starting point of our approach is to measure the output at two tapping points, $A_{l}$ and $A_{3}$, individually in order to get access to the voltage magnitude at each point. The two outputs are then connected to the power combiner through two cables with nominal $2 \mathrm{~dB}$ of loss at $18 \mathrm{GHz}$, as in Figure 4.5.

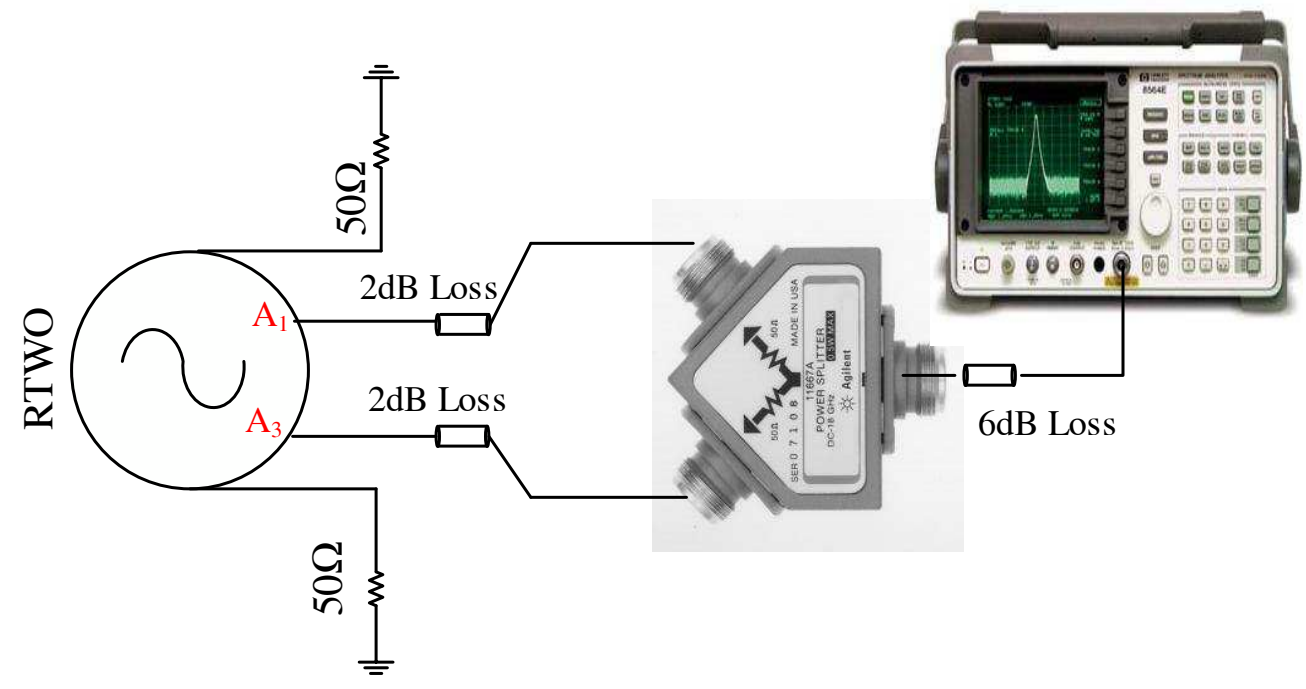

Figure 4.5 Measurement setup used to test our innovative approach

However, to obtain the correct relationship between the combined output power and phase shift at different points, the test setup which includes a power combiner and cables 
has to be carefully calibrated. The mismatch loss between the RTWO outputs and spectrum analyzer should be also taken into account.

\subsubsection{Calibration of the Measurement Setup}

We need to calibrate the following equipments involved in our test setup.

1 11667A power combiner calibration

According to the datasheet of the Agilent 11667A power splitter, this device uses two $50 \Omega$ resistors to split the signal. The nominal insertion loss of the device is $6 \mathrm{~dB}$ while the typical value is between $5.8 \mathrm{~dB}$ and $7.8 \mathrm{~dB}$ for the $8 \mathrm{GHz}-18 \mathrm{GHz}$ band.

In order to calibrate the available power combiner in our lab, we have used a high frequency signal generator, cables with known loss and a $50 \Omega$ termination. With these, the power combiner shows $7.5 \mathrm{~dB}$ of loss from one arm and $7.66 \mathrm{~dB}$ of loss from the other arm.

\section{Cable calibration}

We have used a pair of length-matched cables to connect the outputs of the RTWO to the power combiner. Our measurement results show $2 \mathrm{~dB}$ of loss and approximately $4.17 \mathrm{~ns} / \mathrm{m}$ of time delay for each cable at $18 \mathrm{GHz}$.

The individual cable we have used to connect the output of the power combiner to the spectrum analyzer shown in Figure 4.5, has $6 \mathrm{~dB}$ of loss at $18 \mathrm{GHz}$. The time delay of 
this last piece of cable is not important since we are only measuring the magnitude of the combined signals.

3 Mismatch loss between the RTWO's buffers and the spectrum analyzer

The magnitude of the output impedance of the emitter-follower buffer stages used in our design is $6.5 \Omega$ at $18 \mathrm{GHz}$. Since the output of the RTWO has not been matched to $50 \Omega$, we have to take into account the mismatch loss between the $6.5 \Omega$ source and $50 \Omega$ load according to (4.4) [46].

$$
M L_{d B}=-10 \log _{10}\left(1-\Gamma^{2}\right)
$$

where $\Gamma$ is the magnitude of the reflection coefficient and can be calculated based on (4.5):

$$
\Gamma=\frac{Z_{L}-Z_{S}}{Z_{L}+Z_{S}}
$$

where $Z_{S}$ and $Z_{L}$ are the source and load impedances.

Using (4.4) and (4.5), we find a mismatch loss of $3.90 \mathrm{~dB}$ for our circuit. The $3.90 \mathrm{~dB}$ of power is lost in the system due to the mismatch between the source and the load. Calculation of this value is critical because part of our approach is based on calculations and we need to know the value of the output power the RTWO is generating and the effect of the $50 \Omega$ spectrum analyzer should be calibrated out. 
Therefore, in our case and according to the (4.6), RTWO generates output power of $-8.83 \mathrm{dBm}+3.90 \mathrm{~dB}$ from node $A_{1}$, and $-9.67 \mathrm{dBm}+3.90 \mathrm{~dB}$ from node $A_{3}$.
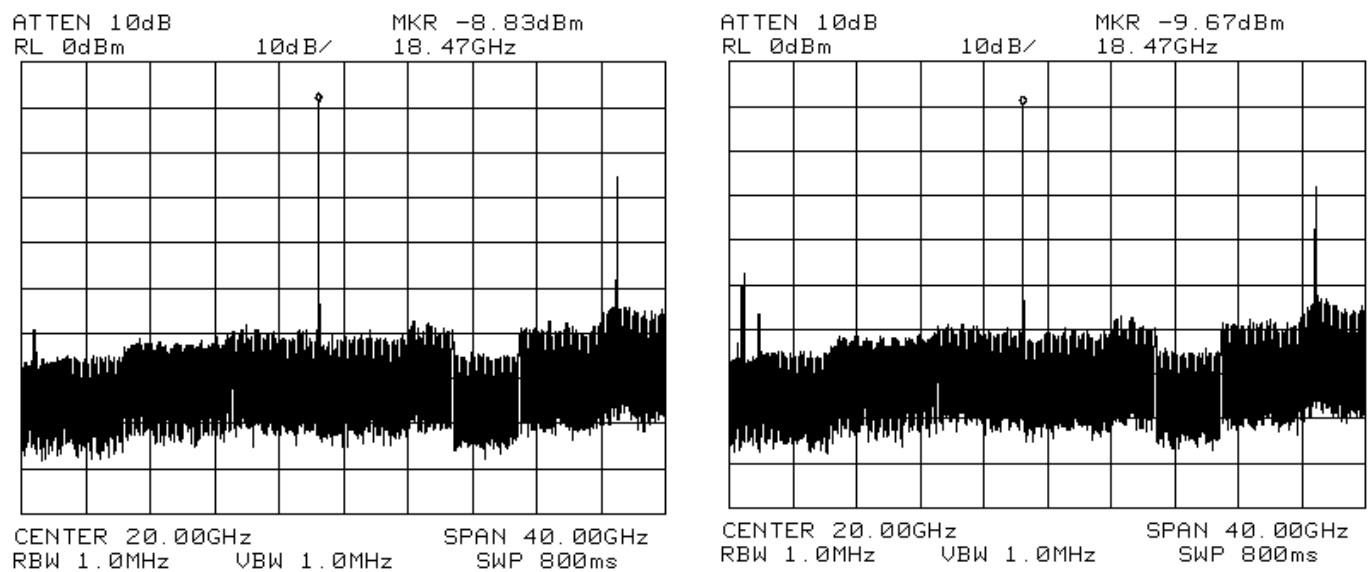

Figure 4.6 The output power of the RTWO at $A_{1}$ and $A_{3}$ nodes

It should be noted that the above measured results are obtained from another RTWO designed for another research work on the correction of an RTWO's phase error using on-chip phase detectors and not the same RTWO as shown in Figure 3.11. That is why the oscillation frequency shown in Figure 4.6 is not the same as Figure 3.12 for varactor tuning voltage of $0 \mathrm{~V}$.

\subsubsection{Voltage Calculation and Power Simulation of the Complete Lossy}

\section{Setup}

Voltage calculation of our proposed method is based on superposition of the lossy setup shown in Figure 4.7. 
After measuring the output power of the RTWO across the $50 \Omega$ spectrum analyzer and taking into account the effect of mismatch loss between the $6.5 \Omega$ output impedance and $50 \Omega$ load, we know that the left segment of the RTWO is generating $-4.93 \mathrm{dBm}$ and the right segment is generating $-5.77 \mathrm{dBm}$ of power.

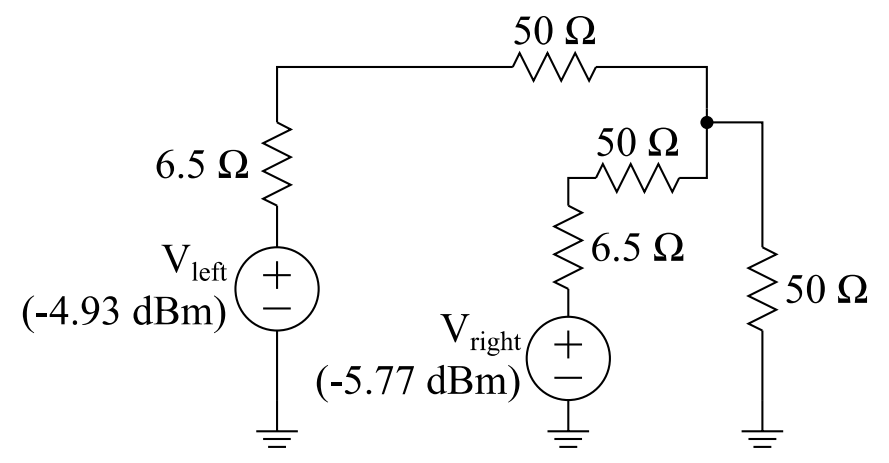

Figure 4.7 Schematic diagram of Figure 4.5 for voltage calculation

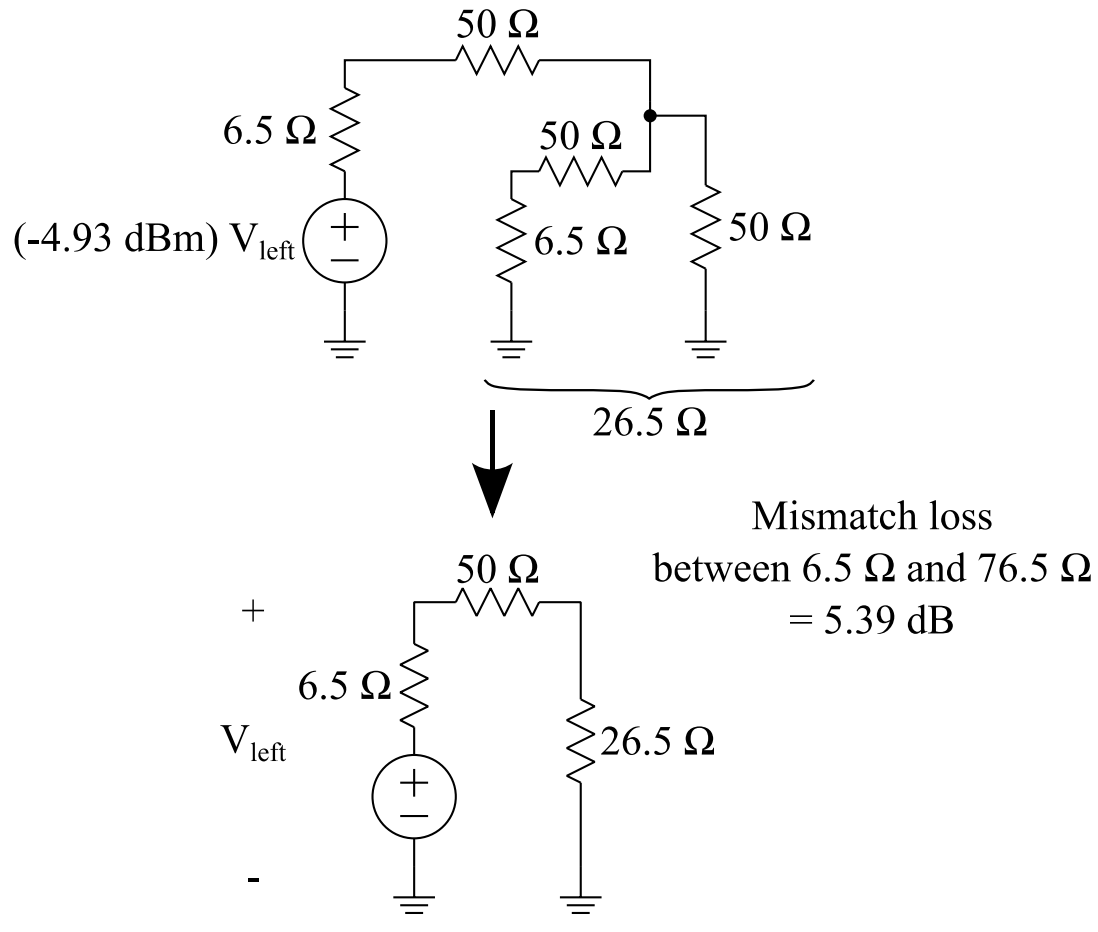

Figure 4.8 Applying superposition and cancelling the effect of the right segment's output 
Applying superposition to calculate the output voltage of the power combiner, we have to take into account the effect of the mismatch loss between $6.5 \Omega$ source and the parallel combination of the rest of the circuit which is $76.5 \Omega$, as shown in Figure 4.8 . This impedance transformation from $6.5 \Omega$ source to $76.5 \Omega$ load corresponds to $5.39 \mathrm{dBm}$ of loss. As a result, in our calculations using superposition we have to assume that the left segment is effectively generating $-4.93 \mathrm{dBm}-5.39 \mathrm{dBm}=-10.32 \mathrm{dBm}$ of power.

Now we can calculate the peak voltage of the power combiner output due to the effect of the left segment only from (4.6):

$$
\begin{gathered}
P=\frac{1}{2} \frac{V_{\text {peak }}^{2}}{R} \\
V_{\text {o_leftseg }}=\frac{26.5}{76.5} \times 1000 \times \sqrt{2 \times 76.5 \times \frac{10^{-10.32 / 10}}{1000}}=41.3 \mathrm{mV}
\end{gathered}
$$

We follow the same procedure for the right segment and cancel the effect of the left segment this time. Taking into account the mismatch loss between $6.5 \Omega$ and $76.5 \Omega$, the right segment is generating $-5.77 \mathrm{dBm}-5.39 \mathrm{dBm}=-11.16 \mathrm{dBm}$ of power which corresponds to an output voltage of:

$$
V_{o_{-} \text {righttseg }}=\frac{26.5}{76.5} \times 1000 \times \sqrt{2 \times 76.5 \times \frac{10^{-11.16 / 10}}{1000}}=37.5 \mathrm{mV}
$$

Now that we have access to the magnitude of two vectors, we can do a simple vector analysis and combine the vectors in order to find the phase shift between them. 
For example, for $90^{\circ}$ of phase shift between left and right segments, the output of the power combiner should be equal to $\sqrt{\left((41.3 \mathrm{mV})^{2}+(37.49 \mathrm{mV})^{2}\right)}$ which results in $55.78 \mathrm{mV}$ or $-15.07 \mathrm{dBm}$ of power across the $50 \Omega$.

Including the effect of the $6 \mathrm{~dB}$-loss cable which is used to connect the output of the power combiner to the input of the spectrum analyzer in our measurement setup, as shown in Figure 4.5, for the exact value of $90^{\circ}$ of phase shift between the two segments, the output power should be equal to $-21.07 \mathrm{dBm}$ based on our hand calculations.

We can also extract the phase shift, $\Delta \varphi$, between the two vectors assuming the phase of one vector is zero and the other vector is shifted in phase by $\Delta \varphi$, as shown in Figure 4.9 and calculated from (4.8).

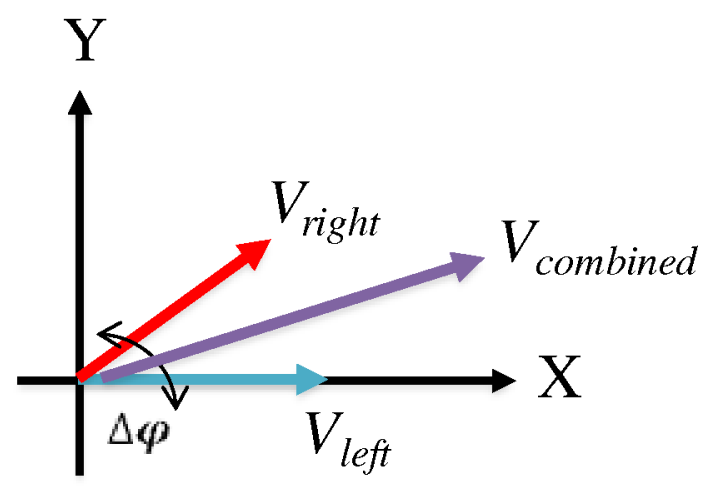

Figure 4.9 Summation of two vectors with $\Delta \varphi^{\circ}$ of phase shift between them

$$
\begin{aligned}
V_{\text {combined }}= & V_{\text {left }}+V_{\text {right }} \\
& =\sqrt{\left(V_{\text {left }}+V_{\text {right }} \cos \Delta \varphi\right)^{2}+\left(V_{\text {right }} \sin \Delta \varphi\right)^{2}} \rightarrow
\end{aligned}
$$




$$
\cos \Delta \varphi=\frac{V_{\text {combined }}{ }^{2}-V_{\text {left }}{ }^{2}-V_{\text {right }}{ }^{2}}{2 V_{\text {left }} V_{\text {right }}}
$$

where $V_{\text {combined }}$ is the output of the power combiner and $V_{\text {left }}$ and $V_{\text {right }}$ are the measured voltages of the left and right segments.

Figure 4.10 shows the schematic diagram used to simulate and find the relationship between the combined output power of the RTWO and the phase shift between the segments considering all the losses and the mismatches explained above.

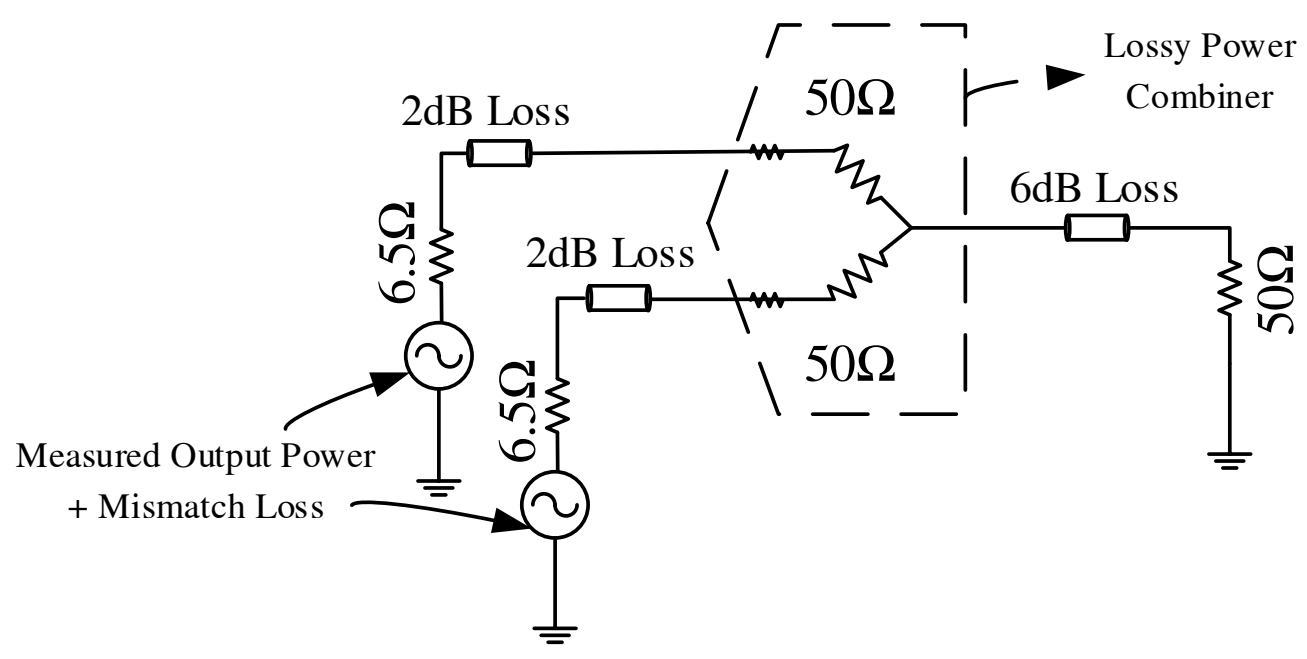

Figure 4.10 Schematic diagram to simulate the output power of the lossy setup

Figure 4.11 shows the simulated results of the combined output power versus the phase shift between the segments including the effect of all nominal losses.

From this figure, for the measured RTWO output powers of $-8.83 \mathrm{dBm}$ and -9.77 $\mathrm{dBm}$ at each tapping point, the combined output power is $-21.07 \mathrm{dBm}$ for phase shift of $\pm 90^{\circ}$, depending on the direction of rotation. This simulated number is perfectly matched our calculated result. 


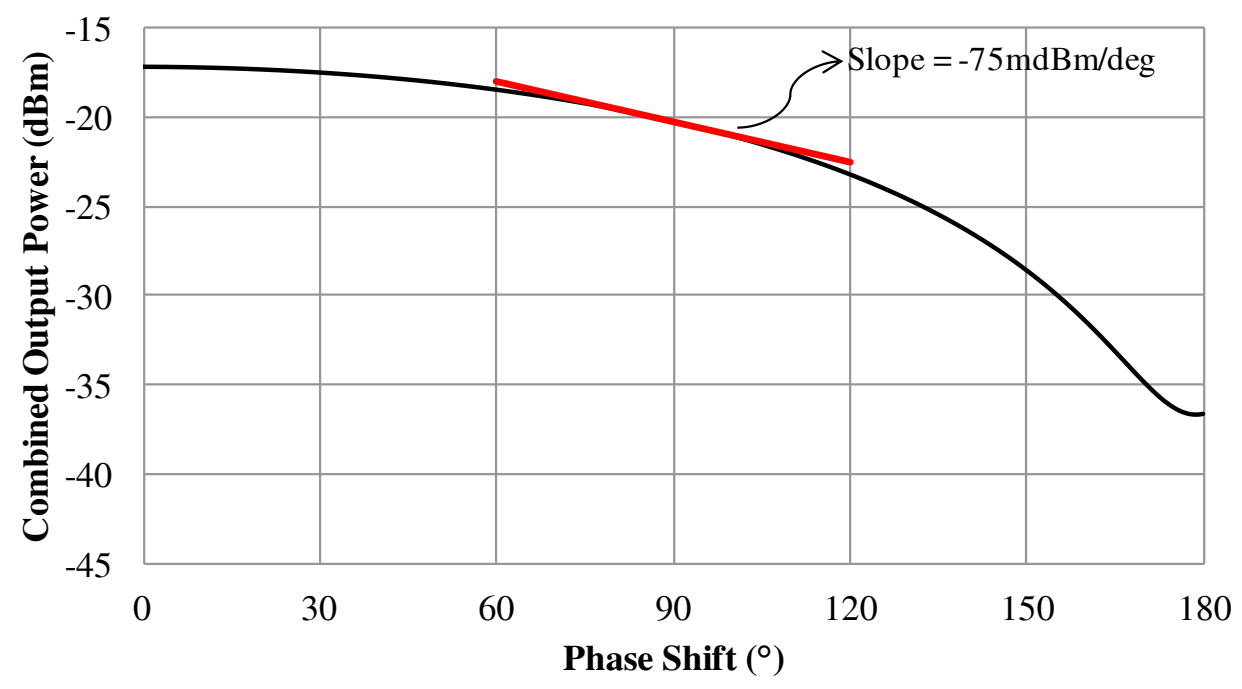

Figure 4.11 Simulated combined output power for different phase shifts

Figure 4.11 also shows that the slope of the tangent line around $90^{\circ}$, between $75^{\circ}$ to $105^{\circ}$, is $-0.075 \mathrm{dBm} / \mathrm{deg}$. This number is small and it shows the sensitivity of our approach to any unaccounted loss in the system. Form the same figure, the slope of tangent line is even smaller for small phase shifts, less than $45^{\circ}$, and perhaps our approach is not accurate enough for small phase shift measurement. However, mathematical calculations show that if the losses of all components are known, measurement of every phase shift would be possible with our approach.

During our calibration procedure, we noticed $0.17 \mathrm{~dB}$ and $0.45 \mathrm{~dB}$ of loss variation for the $2 \mathrm{~dB}$-loss and $6 \mathrm{~dB}$-loss cables, respectively. There is also $3^{\circ}$ of phase tracking mismatch between the arms of the power combiner. Another source of error could be the precision of the spectrum analyzer. Our spectrum analyzer can contribute a maximum of 
$2.3^{\circ}$ of error. The overall calibration uncertainty including all above factors results in a worst-case phase shift error of $\pm 5.5^{\circ}$ around $90^{\circ}$.

Figure 4.12 shows the measured combined output power of the RTWO using the test setup shown in Figure 4.11. From this figure, the measured output power of the combined signals from the $A_{1}$ and $A_{3}$ tapping points at $18 \mathrm{GHz}$, is $-21 \mathrm{dBm}$. By swapping the arms of the power combiner we can cancel out the effect of phase tracking between the arms.

According to Figure 4.12, $-21 \mathrm{dBm}$ of combined output power corresponds to a nominal phase shift of $91.75^{\circ}$. Being able to accurately calibrate the setup system, including three cables and the power combiner, our approach should be as accurate as any other phase measurement approach at high frequencies.

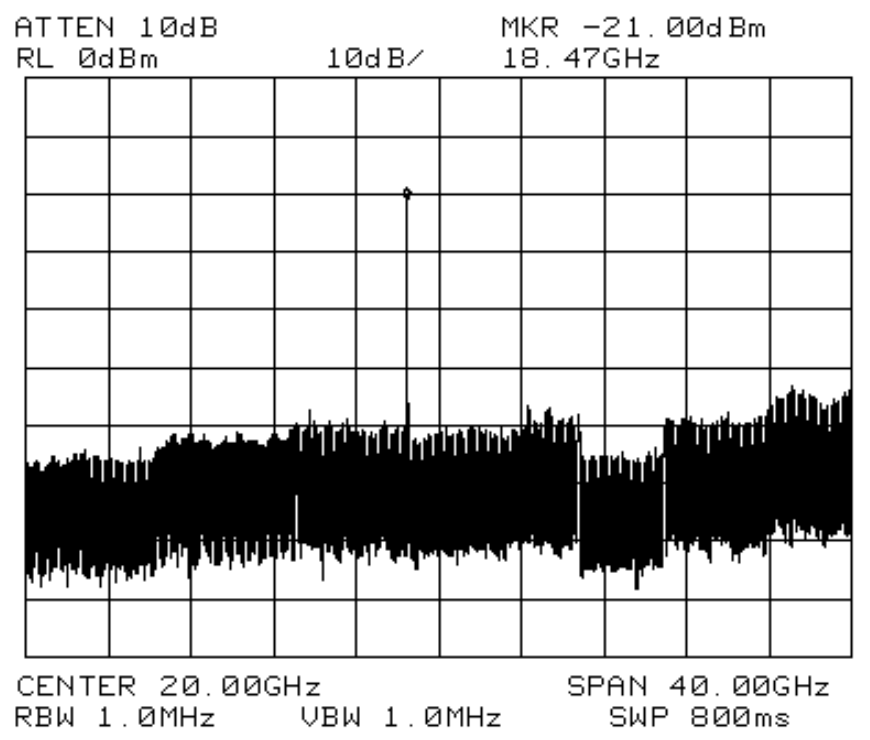

Figure 4.12 Measured combined output powers from $A_{1}$ and $A_{3}$ 
In order to validate the proposed approach, we have used two external frequency mixers to mix down the oscillation frequency to $500 \mathrm{MHz}$. From Figure 4.13, the measured RTWO phase shift between nodes $A_{1}$ and $A_{3}$ is $86.5^{\circ}$, agreeing with the new technique, considering the expected worst case error.

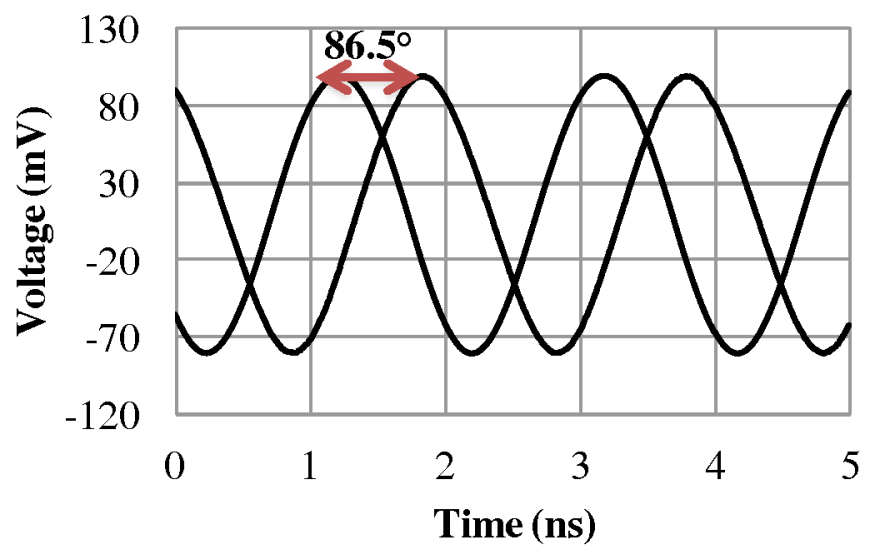

Figure 4.13 Time domain result

These results also demonstrate that our RTWO is working as a multi-phase signal generator with a relatively small phase error.

\subsection{Summary}

There are different off-chip techniques to measure the phase shift between the high frequency signals. The most unambiguous but not easily available approach is to measure the time delay between the signals using a high frequency multichannel, real time oscilloscope. 
Here, at Carleton University, we have access to a HP 54759A high-bandwidth digitizing oscilloscope but these digitizing oscilloscopes need a trigger signal to operate and in our case the maximum triggering signal frequency is $12 \mathrm{GHz}$ while our RTWO is providing $18 \mathrm{GHz}$ signals.

We had to devise a new approach to measure the phase shifts of our RTWO which is both accurate and also possible with our lab equipment.

Using our innovative but simple phase measurement approach, we can first easily determine whether the signal is standing or travelling. Furthermore, without access to a high frequency oscilloscope, like in our case, this measurement technique combined with careful Monte Carlo and EM simulations should give us a correct answer for the phase shift between the segments.

In order to measure the phase shift at high frequency accurately, all techniques require a rigorous calibration process to be able to make an accurate phase measurement. A precise phase measurement using our approach is sensitive to any unaccounted loss in the measurement setup and the situation is getting worse for small phase shift measurement but our approach is still practical and possible in every lab.

A possible extension of this technique would be to do the summation on-chip. If the standing mode condition is detected it might even be possible to provide feedback, e.g. increased bias current for stronger coupling, to force the oscillator into the traveling mode. However, if on-chip, a phase detector may be a more appropriate option. 


\section{Chapter 5 Ku-band Integrated}

\section{Building Blocks for a Phased-Array}

\section{Transmitter Design}

In this chapter, the design methodology and layout considerations of different building blocks needed for the design of a Ku-band integrated phased-array transmitter are presented. As discussed in Chapter 2, in order to design an integrated phased-array transmitter utilizing LO phase shifting, a component which can generate multiple output phases and also a type of distribution network, or power splitter, are needed to create and deliver the multiple outputs having constant phase shifts to a phase selector unit and finally to the antennas.

The phase selector unit in a phased-array transmitter is responsible for selecting an appropriate phase shift in order to effectively radiate in the desired direction with higher power. Finally, the selected signals from the phase selector unit have to be amplified before being transmitted by the antenna array and power amplifiers have to be employed before each antenna in the antenna array.

In order to construct such an integrated Ku-band transmitter, we have designed the following building blocks: 
1) an RTWO;

2) a high frequency distribution network;

3) switches;

4) a power amplifier; and

5) a coplanar patch antenna.

Error! Reference source not found. shows the four-element array transmitter we have targeted to design for this research. As can be seen from this figure, all the antennas should be aligned on an axis, either the $\mathrm{X}$-axis or $\mathrm{Y}$-axis, in order to be fed by power amplifiers and to radiate in the desired direction.

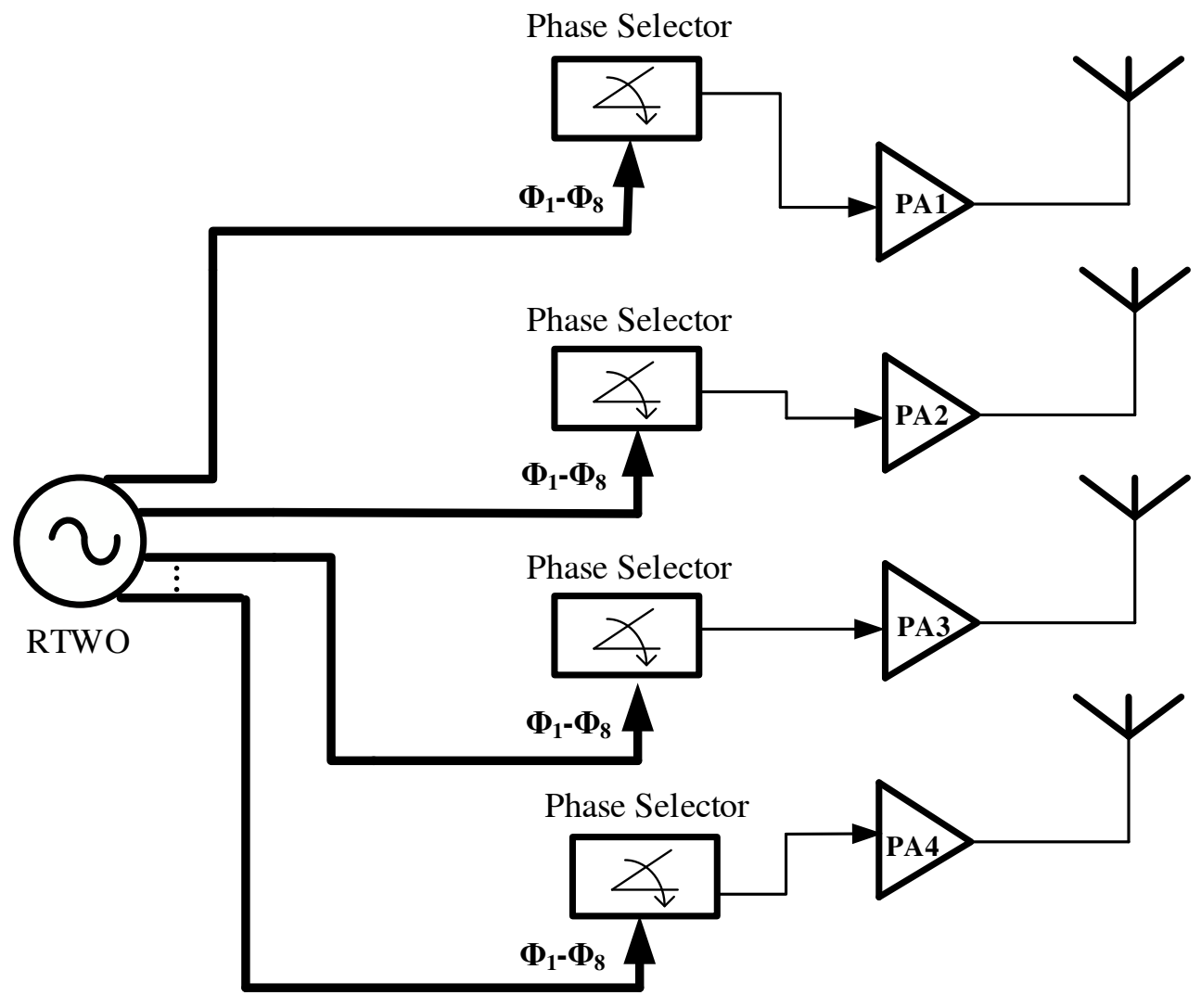

Figure 5.1 The 4-element array transmitter utilizing an RTWO 
Equation (2.25) shows the relationship between the radiation angle of the antennas and the relative phase shifts between the elements in an $n$-element array.

In Error! Reference source not found., the phase shift, $\Delta \varphi$, delivered to each antenna should be constant in order to do beam-forming at the defined direction based on (2.25). The RTWO is capable of providing a minimum $\Delta \varphi$ of $45^{\circ}$ and a maximum $\Delta \varphi$ of $135^{\circ}$ to be delivered to all four elements. For the first case, the elements might receive the signals shifted in phase by $0^{\circ}, 45^{\circ}, 90^{\circ}, 135^{\circ}$ and for the second case the signals may have the phases of $0^{\circ}, 135^{\circ}, 270^{\circ}$, and $45^{\circ}$.

In the following sub-sections we are briefly explaining the design, layout implementation and measurement results of the above building blocks.

\subsection{RTWO}

The detailed study of the design, layout considerations and also measurement results of a multiphase Ku-band RTWO, have been provided in Chapters 3 and 4. Our designed RTWO provides eight different output phases in steps of $45^{\circ}$ and it achieves a tuning range of $1.2 \mathrm{GHz}$ for the measured output power of $-6.5 \mathrm{dBm}$ at $17 \mathrm{GHz}$ without any noticeable output power variation across the tuning range. The measured phase noise of the RTWO is $-104.2 \mathrm{dBc} / \mathrm{Hz}$ at a $1 \mathrm{MHz}$ offset from the carrier frequency.

The functionality and performance of the RTWO being loaded with the input impedance of a distribution network, instead of normal $50 \Omega$ loading, have been checked and evaluated in simulation and we have not noticed significant changes in the 
performance. Furthermore, the measurement results shown in Chapter 3 are obtained from an RTWO terminated with the input impedance of a distribution network which will be explained in this chapter. As a result, the centre frequency and output power of the RTWO should be the same as what we have measured in Chapter 3, while employed in the full system.

\subsection{Distribution Network}

As discussed in Chapter 3, the RTWO has been designed to give access to output phases on the top, bottom, right and left sides of the resonator while as seen in Error! Reference source not found., all the outputs of the RTWO have to be delivered to the antenna array along the $\mathrm{Y}$-axis and on the right side of the system. This means that a custom-designed distribution network operating at $17.5 \mathrm{GHz}$ is required in order to convey the outputs of the RTWO to the antennas.

Figure 5.2 shows the typical H-tree network normally used for symmetric signal distribution, as used in [9] and [36].

For both [9] and [36], the adoption of an H-tree network with its typical geometry, shown in Figure 5.2, is quite convenient. This is due to the fact that their multiphase signal generator, ring oscillator, is laid out for being engaged in a phased-array application since the VCO's output access are only on the left and right sides of the oscillator geometry. More importantly, these researchers have designed a four-element array transmitter applying antenna array placement on both directions of the X-axis, left 
and right sides of the system, and the H-tree network with its typical shape is the best candidate for their signal distribution.

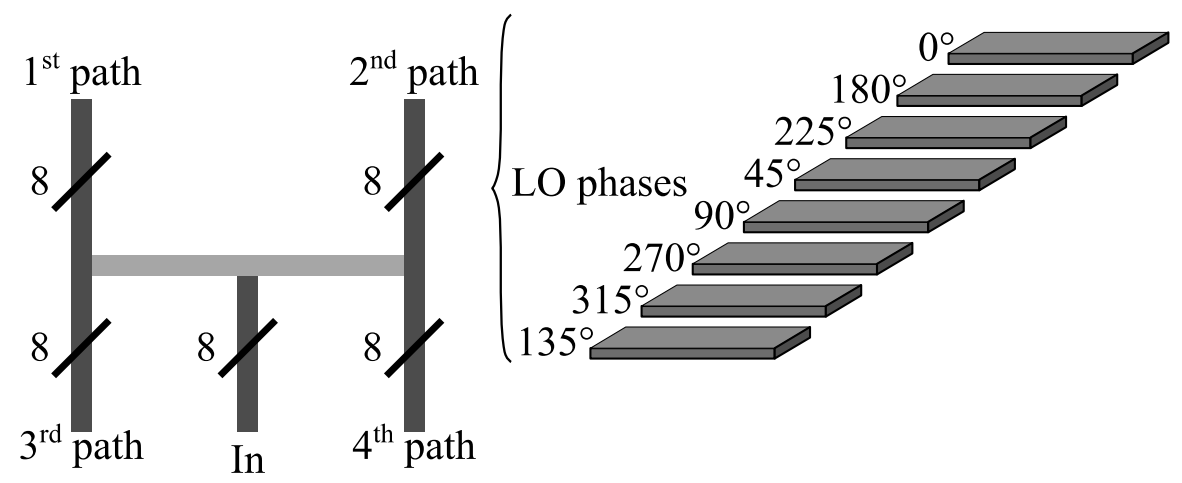

Figure 5.2 Typical H-tree distribution network

However, these references have not reported any measurement results using physical off-chip antennas, for which all of the antennas have to be placed along the same axis to couple to each other for beam-forming, and the measurements have been performed with the aid of off-chip phase shifters to effectively combine the outputs of all four elements and extract the radiation angle from the output of the phase shifters. This is the same idea as we have used for the phase shift measurement of the RTWO and the details have been discussed in Chapter 4.

In our case, where we have an RTWO with output access at different positions on the resonator and where it is desired to place all four off-chip antennas along the Y-axis, the typical H-tree network is not practical because of the amount of phase error it can potentially add to the outputs of the RTWO with shorter distance to the antenna array. 
Our first attempt at a solution involved the design of an $\mathrm{H}$-tree network with meandered arms to be able to add more delay to shorter arms to the antennas, the RTWO's right segment's outputs in our case, and maintain the phase while ending the arms of the H-tree network at the same point and all along the same axis.

Figure 5.3 shows the layout of the H-tree distribution network with meandered arms. From this figure, all eight output phases of the RTWO have been distributed to four different positions along the same axis and with the same physical length.

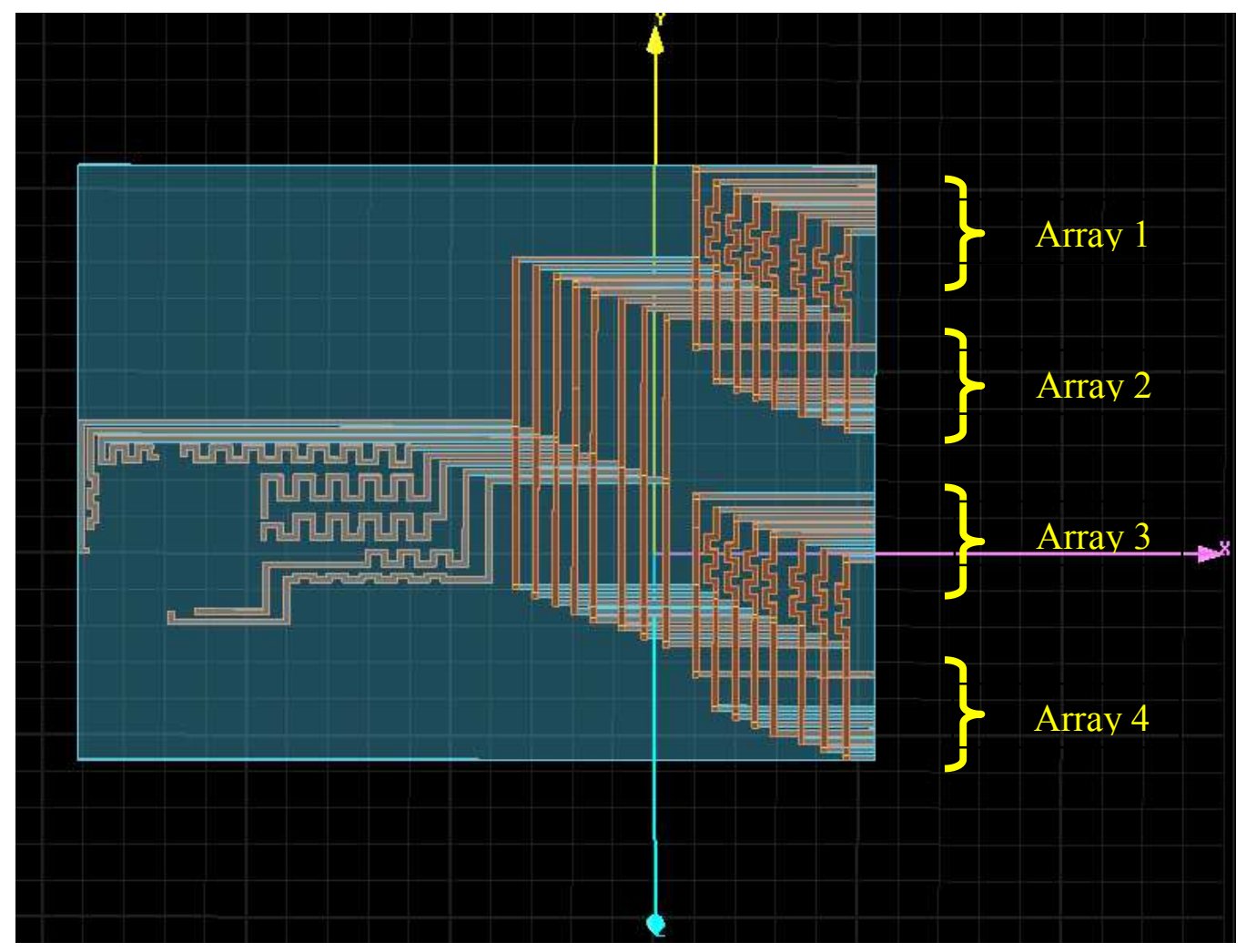

Figure 5.3 Meandered H-tree network

Figure 5.4 shows our EM simulation results for the distribution network realized in Figure 5.3! As can be seen from this figure there is close to $15^{\circ}$ of phase error between 
the output of each element and this error goes up to $24.5^{\circ}$ between the same signals routed to different elements, as illustrated in Figure 5.4.

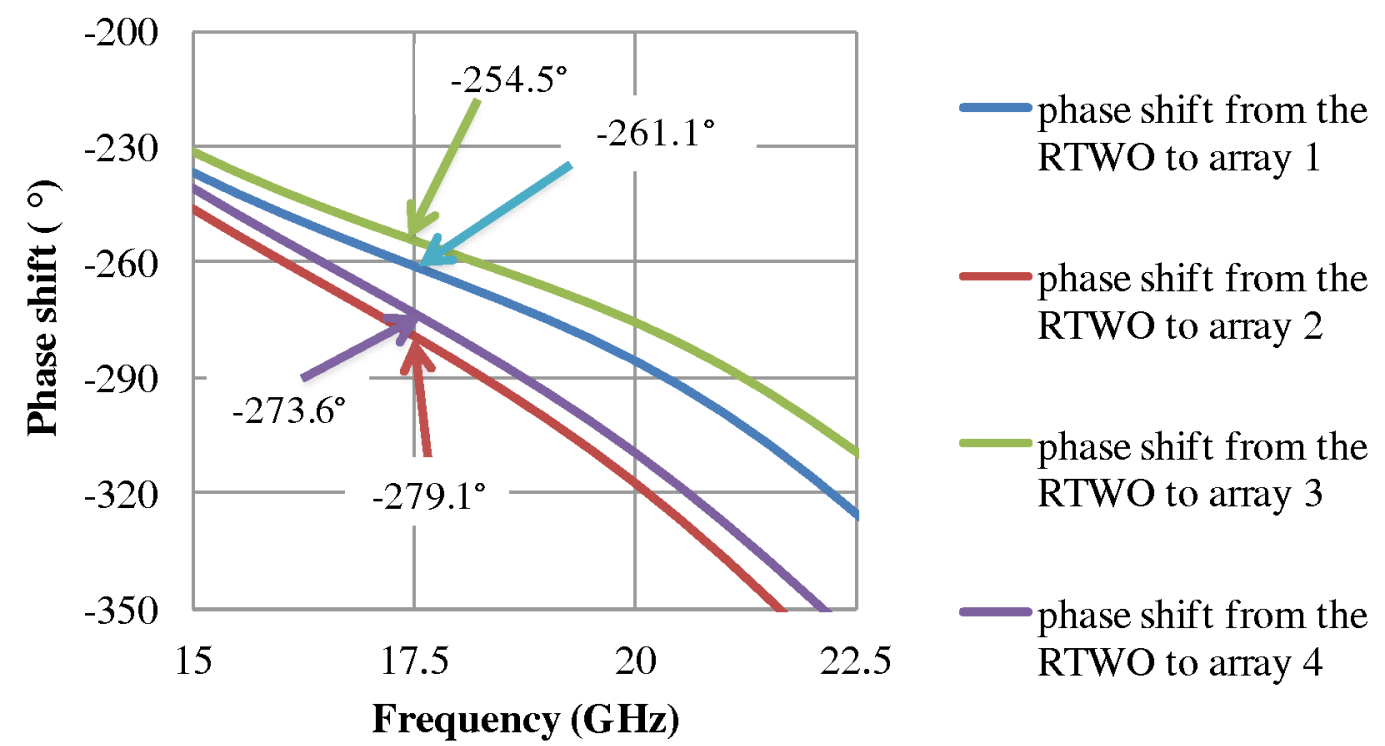

Figure 5.4 HFSS simulation results of the meandered H-tree network

Further and in-depth investigations using both HFSS and Agilent's Momentum, illustrated that this huge amount of phase error is due to the corners and discontinuities of the meandered lines and also crossing and proximity of different arms holding high frequency signals.

In order to fix this problem, we laid-out a distribution network which delivers all eight output phases to four different antennas all along the same axis while it contains almost no meandering and minimum number of arms' crossing.

Figure 5.5 and Figure 5.6 show our custom-designed distribution network and its EM simulation results, respectively. 


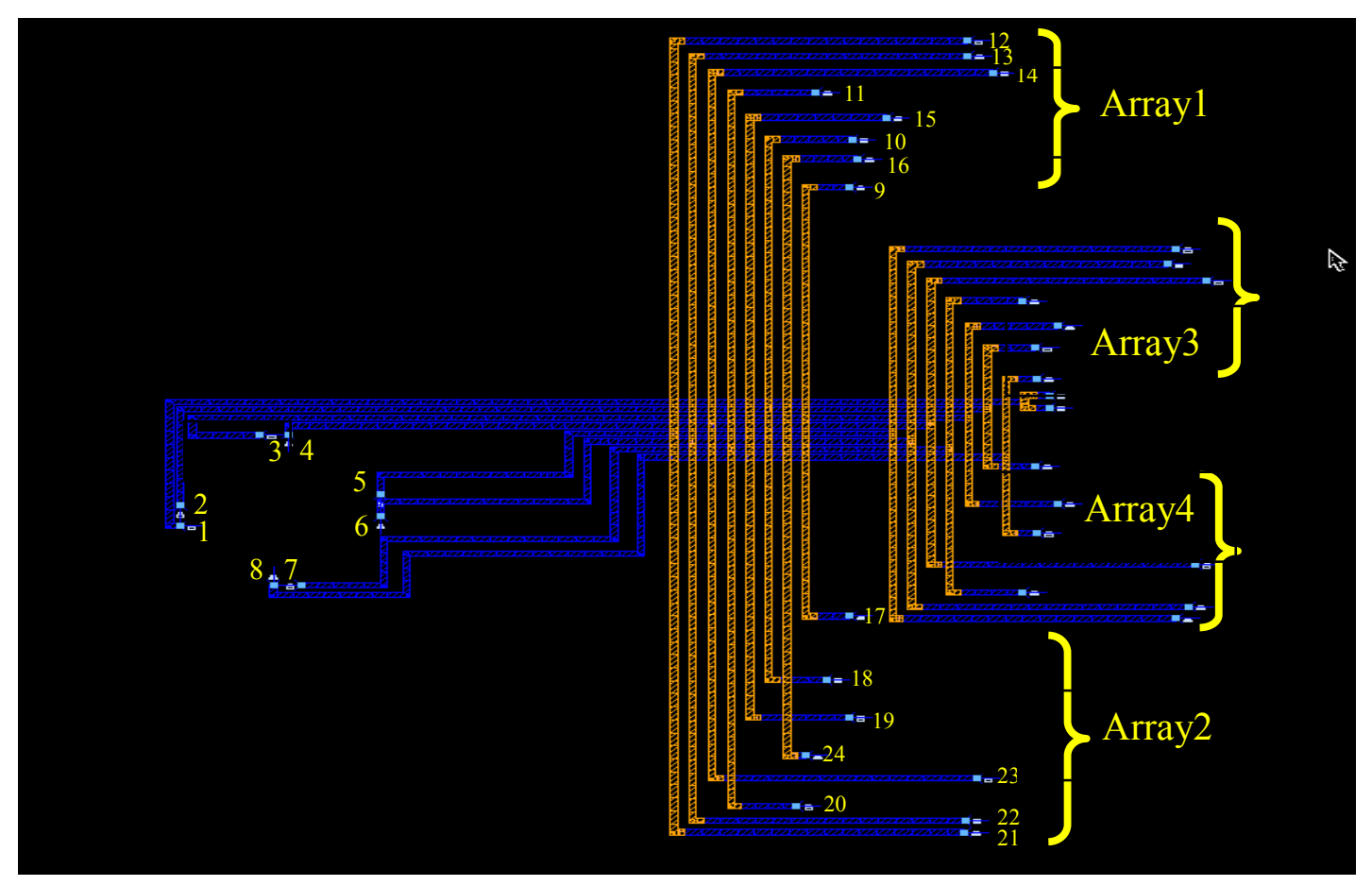

Figure 5.5 Our designed distribution network for four-element array

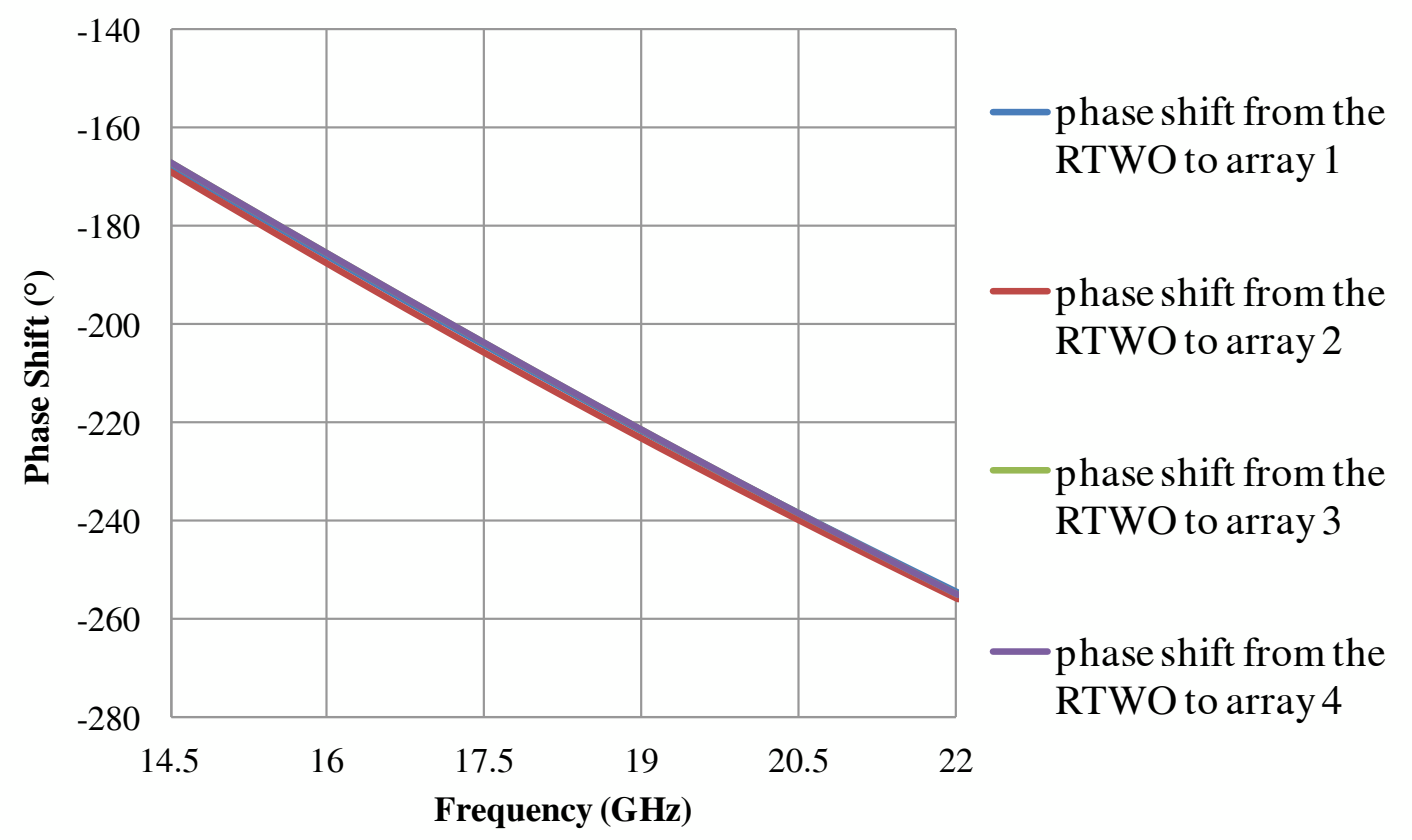

Figure 5.6 EM simulation results of our distribution network 
From Figure 5.6, the maximum phase error between the outputs of the same element is $6^{\circ}$ and for the same signal routed to different elements this error reduces to $3^{\circ}$. There is also close to $1 \mathrm{~dB}$ of amplitude mismatch between the different arms of the network which can degrade the beam forming resolution of the transmitter.

The only problem with this network for a unidirectional phase-array system is the fact that different arms are not ending at the same point. However, this problem can be alleviated in the layout design of the following building blocks including an analog multiplexer and power amplifier matching network.

The total die size of the fully integrated four-element array transmitter utilizing our distribution network was estimated to be $36 \mathrm{~mm}^{2}$. Due to this large silicon area, we changed our design to a two-element array transmitter, instead of a four-element array, which was initially targeted to be designed. Adding a beam-forming capability to a transmitter should be possible with two-elements and this is enough to show the proof of the concept.

Finally, we optimized our distribution network for a two-element array signal distribution. The network it is realized by microstrip-line structures with a signal line being laid out using $30 \mu \mathrm{m}$ top two metal layers and the first metal layer $\left(M_{l}\right)$ used as a ground plane. The distribution network is designed for relatively low characteristic impedance, $Z_{0}$, since we need a matching network between the output of the RTWO's buffers, which is $6.5 \Omega$, and the input of the switching bank which will be explained in the next sub-section. 
The characteristic impedance and length of each arm of the distribution network from the RTWO outputs to the input of the switching bank is $25 \Omega$ and $3 \lambda / 8$, respectively. These numbers have been used for matching purposes.

The final EM simulation results of our distribution network for the two-element array are shown in Figure 5.7. From this figure the average power loss of each arm is $9.3 \mathrm{~dB}$, loading the switching bank, with $1 \mathrm{~dB}$ of amplitude mismatch and $3^{\circ}$ of phase error between the arms.

We have optimized the distribution network more for phase accuracy than for the power loss or amplitude mismatch. This is due to the fact that we will compensate the loss of the network with the gain of a power amplifier used in each element and the amplitude mismatch is not the main concern in phased-array transmitters employing LO phase shifting.

Reducing the size of the distribution network is possible by using the slow wave propagation concept like the technique used in [47], where strips of floating metals were used under the coplanar signal lines in order to shrink the length of the line. However, in order to get noticeable reductions using this technique the CPW structure has to be used which is naturally big and might not ultimately help us with size reduction.

The method used in [48] is more practical in our case. In order to take advantage of slow wave propagation and reduce the size of the signal line, the authors have designed a periodic $L C$ network, by periodically cutting the ground plane under the signal line to increase the inductance and adding MIM capacitors to the structure, to store magnetic and 
electric energies spatially separately and they have obtained the slow wave factor of 4.3 in Ku-band.
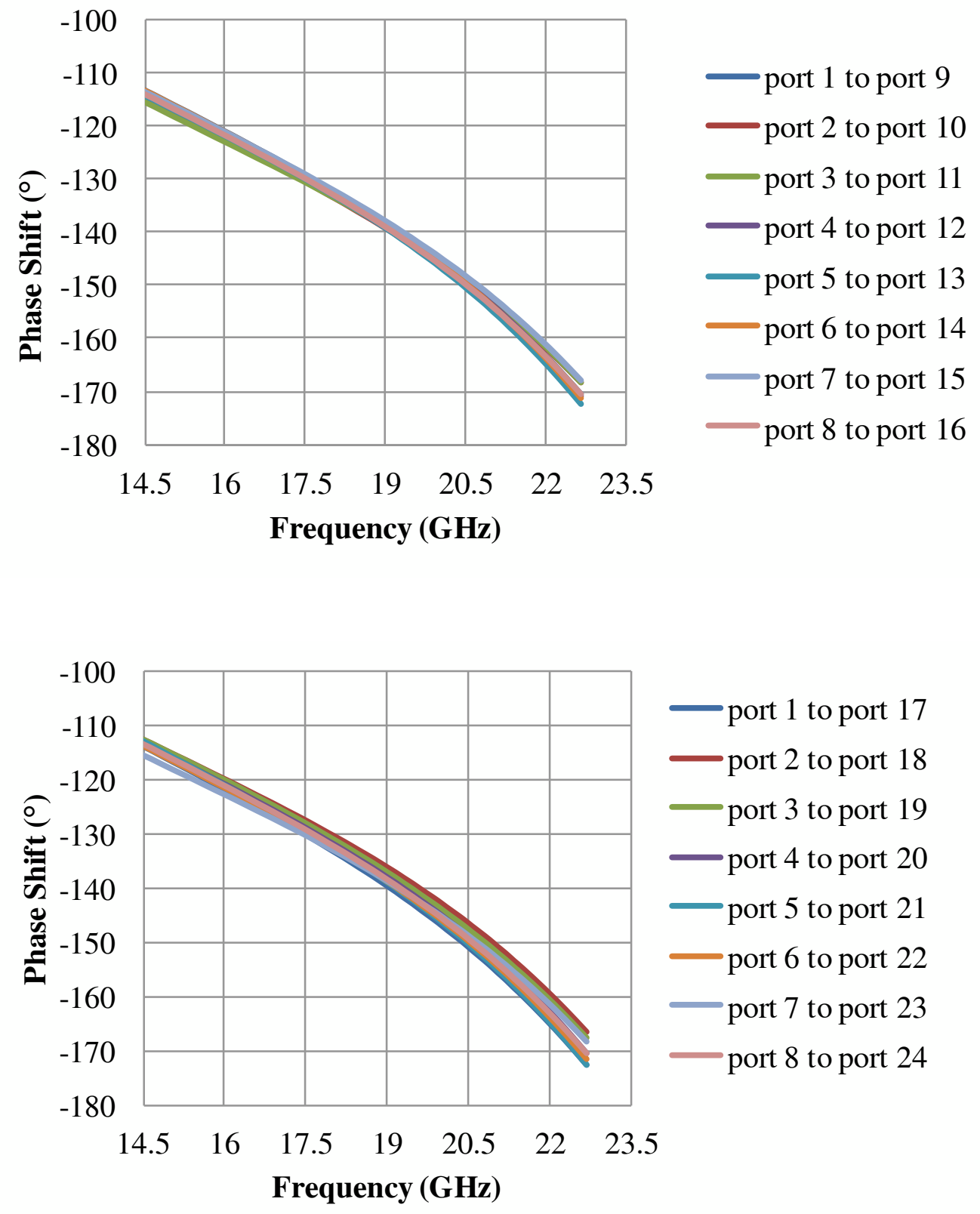

Figure 5.7 EM simulation results of the distribution network for two-element array signal distribution 
Our distribution network might not be practical for monolithic phased-array transmitter designs with more than four elements [49]-[50], aligned on the same axis but still comparable in size and especially performance, with standard H-tree networks.

\subsection{Switches}

As shown in Figure 5.1, in order to enable the beam forming with an antenna array, the outputs of the RTWO have to be provided to a phase selector unit in each element in a symmetric fashion.

For the phase selector unit, we have designed a bank of switches, eight switches for each element, to select the signal with the desired phase shift. This signal will finally be amplified and fed to the antenna.

A total of 16 symmetrical switches have been designed for our two-element array transmitter. An analog multiplexer is needed before each amplifier to combine the switches' outputs in each element and pass the correct phase to the antenna array in order to do beam-forming.

The key FOMs for a switch are insertion loss, isolation, and power-handling. We have employed a body-floating technique for the design of CMOS high frequency switches in order to improve the switch isolation [51]. The effect of body-floating technique is shown for both $\mathrm{ON}$ and OFF switches in Figure 5.8 and Figure 5.9, respectively. 
As can be seen in these figures, the body of the transistor is connected to a $10 \mathrm{~K} \Omega$ resistor instead of being directly connected to the source of the transistor.

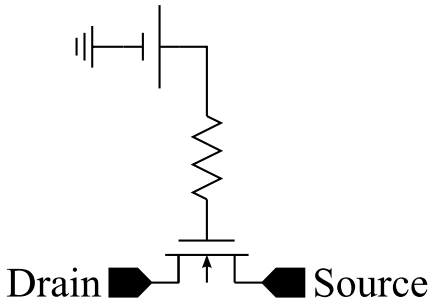

(a)

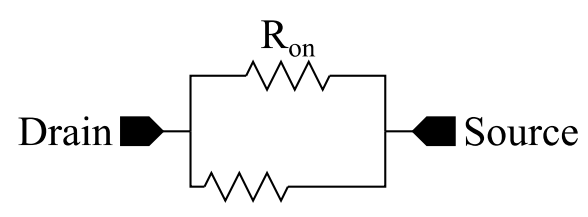

(c)

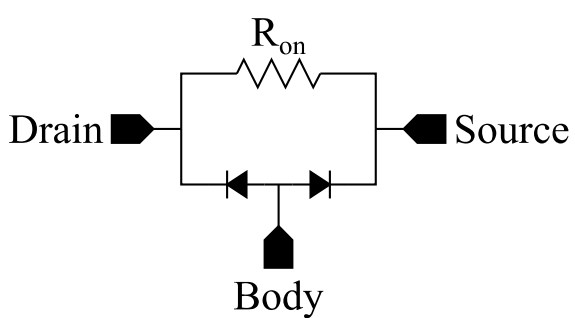

(b)

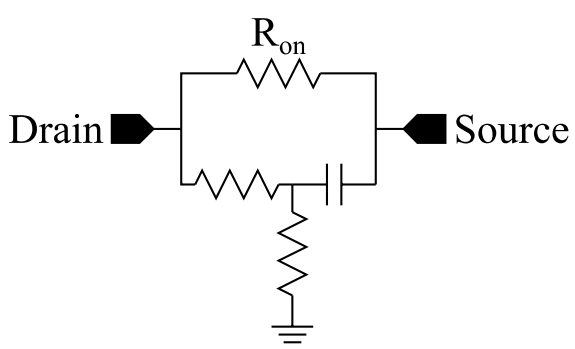

(d)

Figure 5.8 Circuit schematic of a series transistor in ON state

For high power applications, as the input power increases it can turn on the diode between the drain and body of the transistor. The overall input impedance of the transistor, if body and source are tied together, will be the parallel combination of $R_{o n}$ and parasitic resistance of this $\mathrm{ON}$ diode. This changes the dynamic load line of the switch and degrades the power handling performance of the device.

However, as can be seen in Figure 5.8(d), using a body-floating technique with a large resistor keeps the $R_{o n}$ of the switch almost constant even if the drain to body diode turns on. 
Figure 5.9 shows the effect of the same technique on the shunt switch in the OFF state.

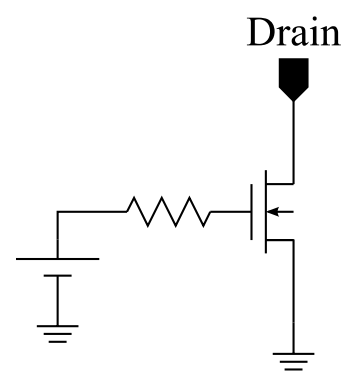

(a)

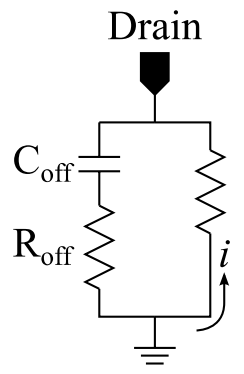

(c)

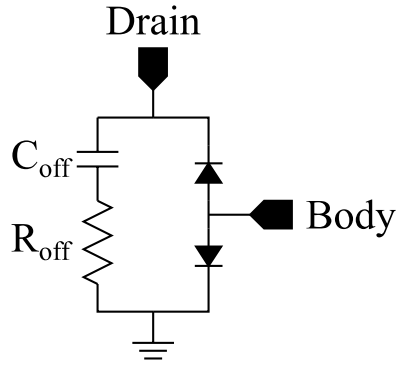

(b)

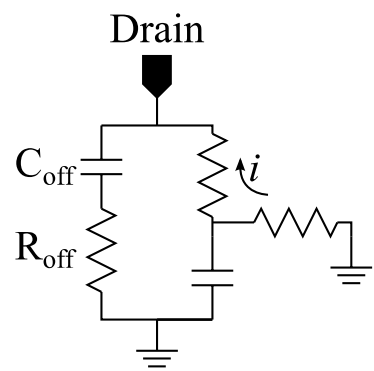

(d)

Figure 5.9 Circuit schematic of a shunt transistor in OFF state

From this figure, without the body-floating technique, high input power can turn on the diode between drain and body of the transistor in the OFF stage and a high amount of current can flow from the ground to the drain terminal of the transistor. However, using a $10 \mathrm{~K} \Omega$ resistor to connect the body of the transistor to the ground limits this current and the current from ground to the drain increases slowly. This effect can improve the isolation of the switch.

Figure 5.10 shows the schematic of our designed series/shunt/series switch employing the same technique. We have chosen the transistor width of $150 \mu \mathrm{m}$ for series transistor 
and $170 \mu \mathrm{m}$ for the shunt transistor. However, Qubic4XI design kit from NXP semiconductors does not have a model for triple-well or isolated NMOS transistors which give access to the isolated substrate contacts of the transistors like the ones used in [51] [52]. The body of the normal NMOS transistors is sharing the same silicon substrate with other components and the substrate of these transistors is not readily available to be connected to the desired impedance. As a result, special care was required in the layout of the normal NMOS transistors to make the implementation of the body-floating technique possible.

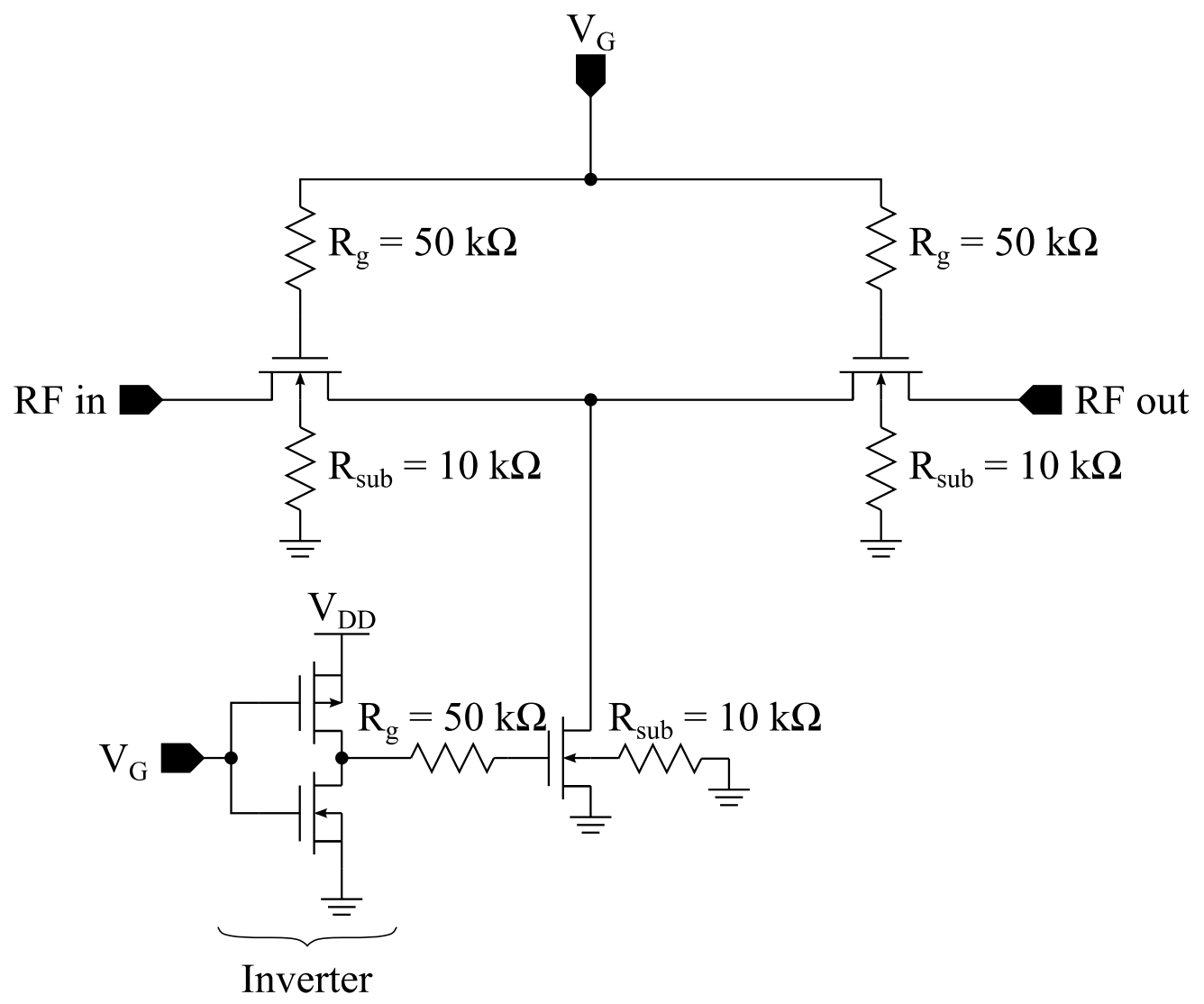

Figure 5.10 Schematic diagram of the proposed series/shunt/series switch with body floating technique 


\subsubsection{Isolating the Substrate Contact of the Switch}

Isolation is a major challenge in electronic circuits and becomes especially challenging in RFIC design due to close proximities, high frequency and a shared silicon substrate.

Reference [53] has shown how adding multiple deep-trench isolation (DTI) layers and ground rings around a pad can improve the isolation.

In order to design our switches without having access to the pcell of an isolated NMOS, we followed the same approach proposed in [53], while in our case a virtual mask layer, called msub, is also required to separate the body of the non-isolated NMOS form the shared silicon substrate and make the layout extractable.

For this, we have inserted a normal ptap ground ring, around other two rings of DTI layers surrounding the non-isolated NMOS. In order to have access to body of the device, the $m s u b$ mask layer has been added within the ptap ring, and dummy ptap vias are used inside the $m s u b$ ring. The dummy ptap is now used to propagate the substrate net of the component to some top levels where it can be connected to any impedance while the outer ptap ring is the real substrate connection and can be treated like a substrate connection of other non-isolated components.

Figure 5.11 shows the layout of the switches realized with the Qubic4XI design kit. The middle transistor is the shunt switch while the two on left and right side of the layout are series switches. 


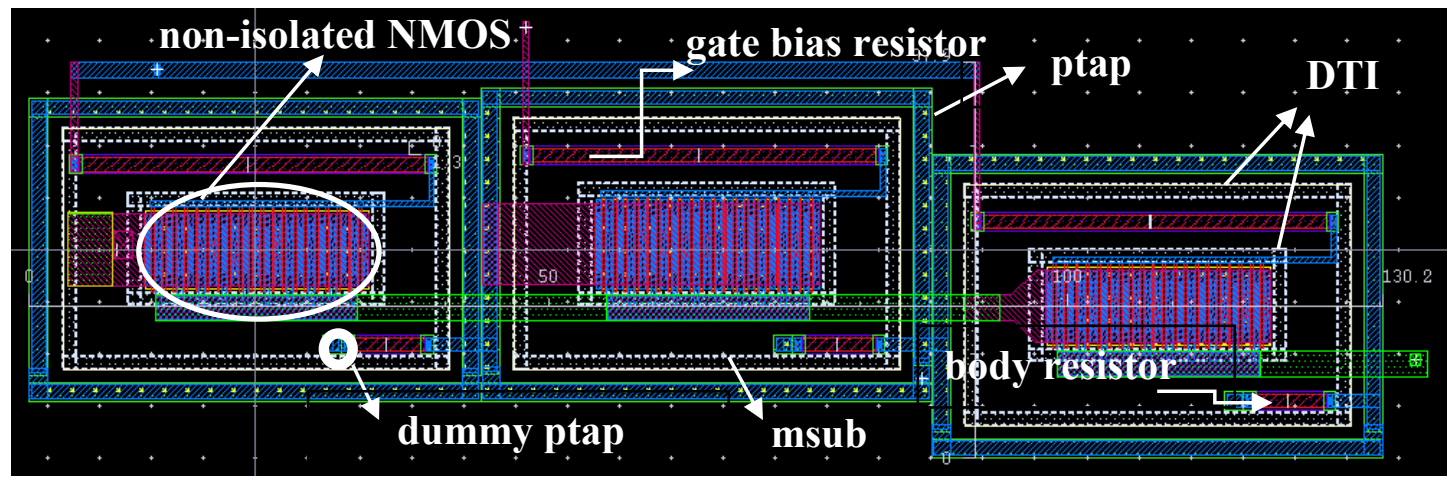

Figure 5.11 Layout of the proposed series/shunt/series switch

The only drawback of this approach is the size of the layout compare to non-isolated NMOS switches. Due to the required minimum spacing between the DTI layers, the layout area of our switch is large, $130 \mu \mathrm{m} \times 38 \mu \mathrm{m}$, considering the fact that there are only 3 transistors involved in the layout.

In general, the size of series and shunt transistors in a switch design has significant influence on the performance of the switch. Smaller series transistor and larger shunt transistor give better isolation while the opposite improves the power handling of the switch. Since in our case the switches have to handle relatively small amount of power to the power amplifiers, $-10 \mathrm{dBm}$, and instead they have to provide good isolation, we have chosen $150 \mu \mathrm{m}$ and $170 \mu \mathrm{m}$ with for series and shunt switches, respectively. The minimum length of CMOS devices in Qubic4XI technology is $0.25 \mu \mathrm{m}$.

An on-chip inverter is designed to provide the complementary voltage of the series transistor to the gate of the shunt transistor, as shown in Figure 5.10. 
In the full phased-array transmitter design, we have used a parallel combination of a real and a dummy switches to keep the loading impedance of the distribution network constant during the phase selection. More details on this will be provided in Chapter 6 .

Figure 5.12 and Figure 5.13 show the performance summary of our switches at $17.5 \mathrm{GHz}$ in $\mathrm{ON}$ and $\mathrm{OFF}$ states, respectively.

From these two figures, the $R L C$ extracted results of the series/shunt/series switches with isolated substrate show insertion loss of $-1.49 \mathrm{~dB}$ and isolation of $-30 \mathrm{~dB}$ at 17.5 GHz which is the centre frequency of the RTWO. The transistor sizes are chosen to give ON-state return loss better than $-10 \mathrm{~dB}$ for matching purposes, as illustrated in Figure 5.12. Our extracted results show an input-referred $1 \mathrm{~dB}$ compression point of $16 \mathrm{dBm}$ which is much higher than the available power in our system.

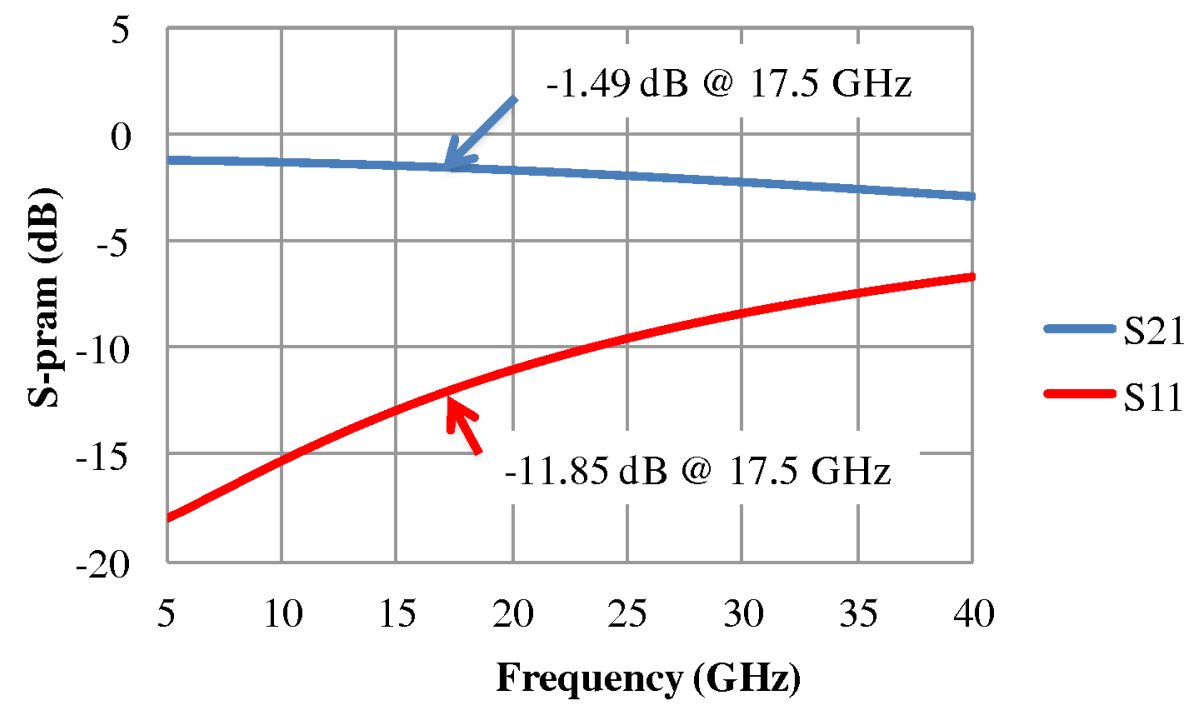

Figure 5.12 Performance summary of the switch in ON state 


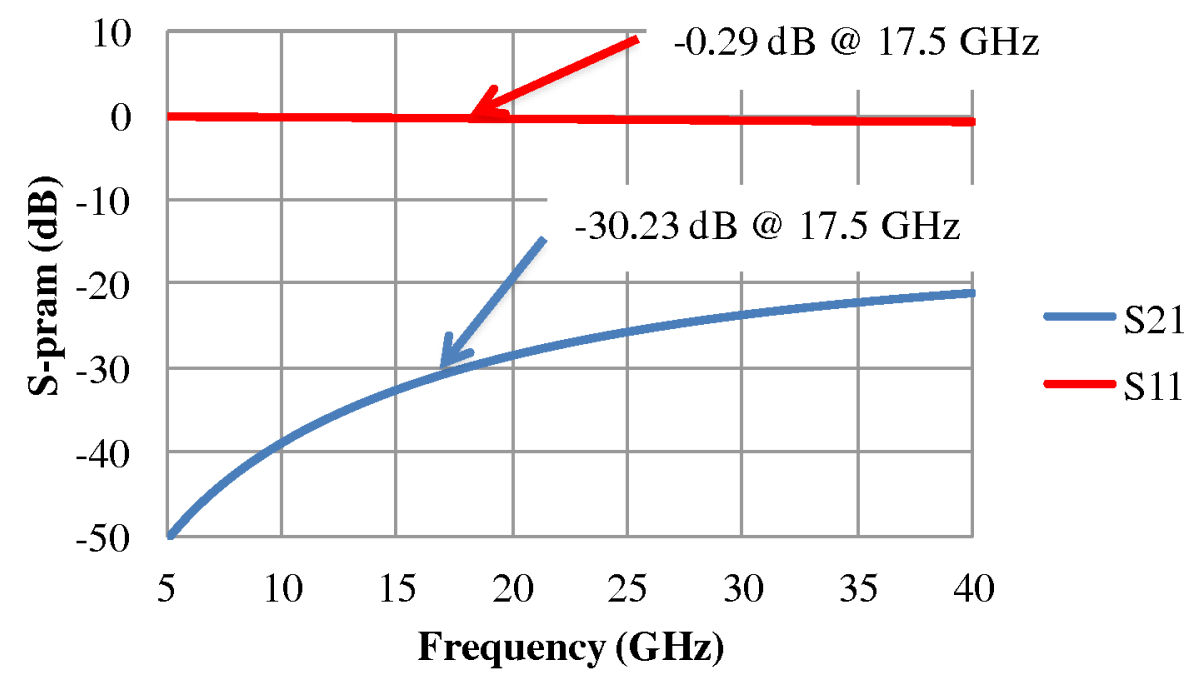

Figure 5.13 Performance summary of the switch in OFF state

Our switch topology is symmetrical and the input and output impedances in both ON and OFF states are the same and they are $44.6-26 \mathrm{j} \Omega$ and $5.4-108 \mathrm{j} \Omega$, respectively. As a result, the parallel combination of real and dummy switches, triggered with complementary gate voltage of the real switches with the aid of on-chip inverter, is used to load each arm of the distribution network. Doing this, the loading impedance of each arm of the distribution network stays constant and equal to $26-29 \mathrm{j} \Omega$.

The most common way of comparing and evaluating a semiconductor switch performance is the $R_{o n} \times C_{o f f}$ product [54]. The on-resistance is the evaluation of the insertion loss, while the off-capacitance affects the isolation. This product is constant and independent of transistor gate width. In our case this value is $962.5 \mathrm{fs}$. 


\subsection{Power Amplifier}

As discussed in earlier sub-sections, designing a phased-array transmitter using LO phase shifting requires some type of distribution network to deliver the output signals to different antennas. Furthermore, in order to have beam-forming capability, a set of switches is required to select the desired phase to be sent to the antenna array. The combination of the distribution network and switching bank introduces a significant amount of loss between the output of the RTWO and the input of the antennas. Hence, use of amplification stages is unavoidable in phased-array transmitters [9], [36] and [55].

However, using many amplification stages on the same die can cause serious stability problems. As a result, we have chosen an RTWO for signal generation because of its high output power, and we have employed a three-stage power amplifier with high gain just before each antenna to compensate for the loss of the distribution network and the switching bank and provide at least $+2 \mathrm{dBm}$ of output power, based on our system specifications, to the antennas.

Most microwave power amplifiers are operated in a compromise between class A, higher linearity, and class B, higher efficiency [56]. We have also chosen class AB power amplifiers for our two-element phased-array transmitter design.

Figure 5.14 shows the schematic diagram of the proposed three-stage class AB power amplifier. As seen from this figure, the PA is single ended and we have chosen a coplanar structure for its layout implementation. 
The coplanar structure has been chosen for the power amplifier implementation due to the fact that the power amplifier has to feed the off-chip antennas on a printed circuit board and a coplanar connection between the die and the PCB is easier to realize. Furthermore, the coplanar structure allows the ground pads of the PA to be more readily available for wire-bonding with less on-chip parasitic inductance compared to microstrip ground via connections.

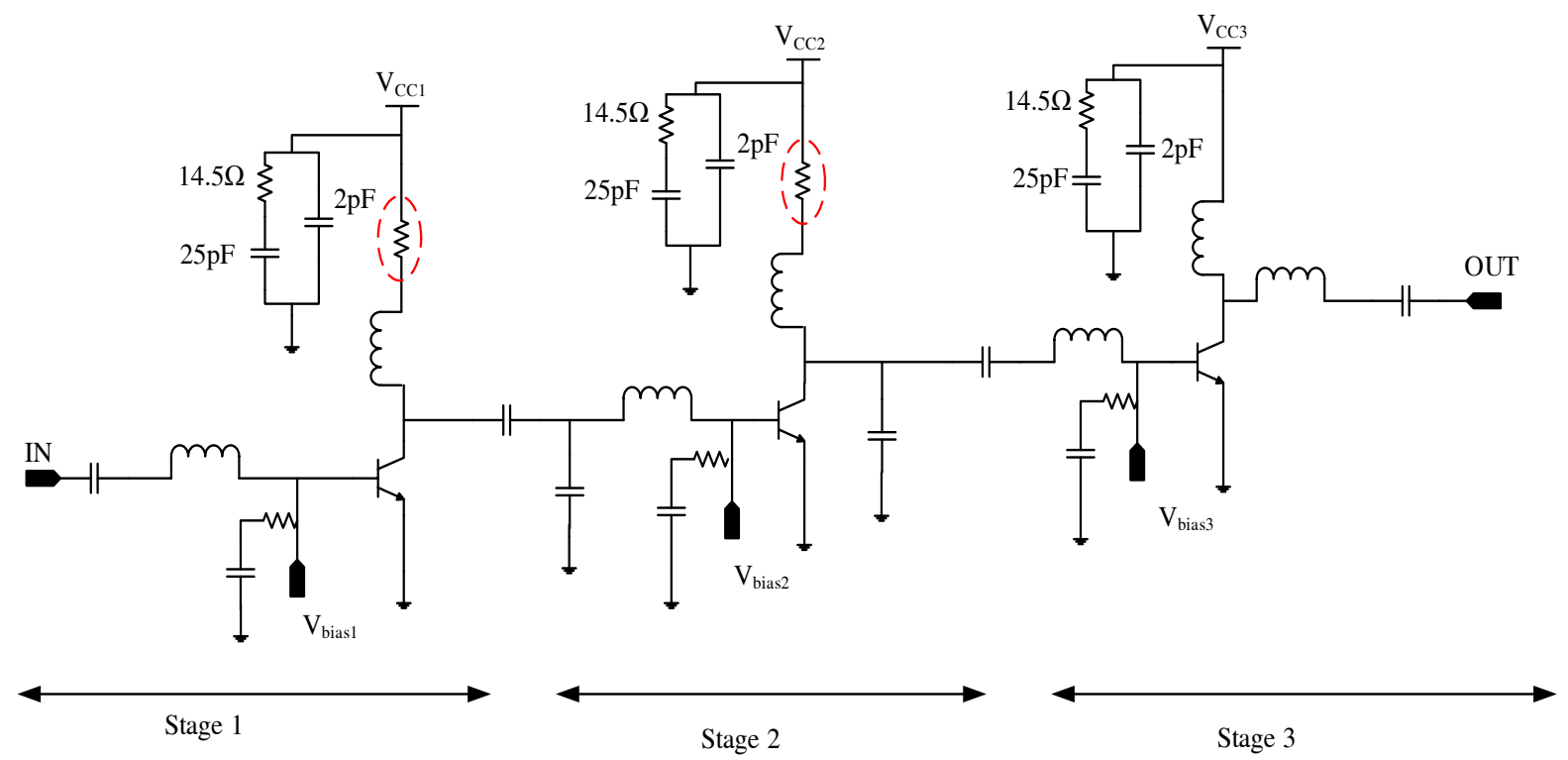

Figure 5.14 Schematic diagram of our three-stage class AB power amplifier

The first two stages of the PA have been designed and matched for high voltage gain and the last stage is utilizing a big transistor for power handling. The matching of the last stage has been done with the aid of a load-pull simulation in order to find the optimum loading for maximum power transfer. The output of each stage is matched to the input of the next following stage. 
Since the power amplifier will drive the off-chip antenna and the silicon die has to be wire-bonded to a PCB, the effect of ground connections and the parasitic inductance of bondwires have been carefully taken into account in every stage of the design.

Figure 5.15 shows the current through the last stage transistor which is biased to operate in class $\mathrm{AB}$ amplification.

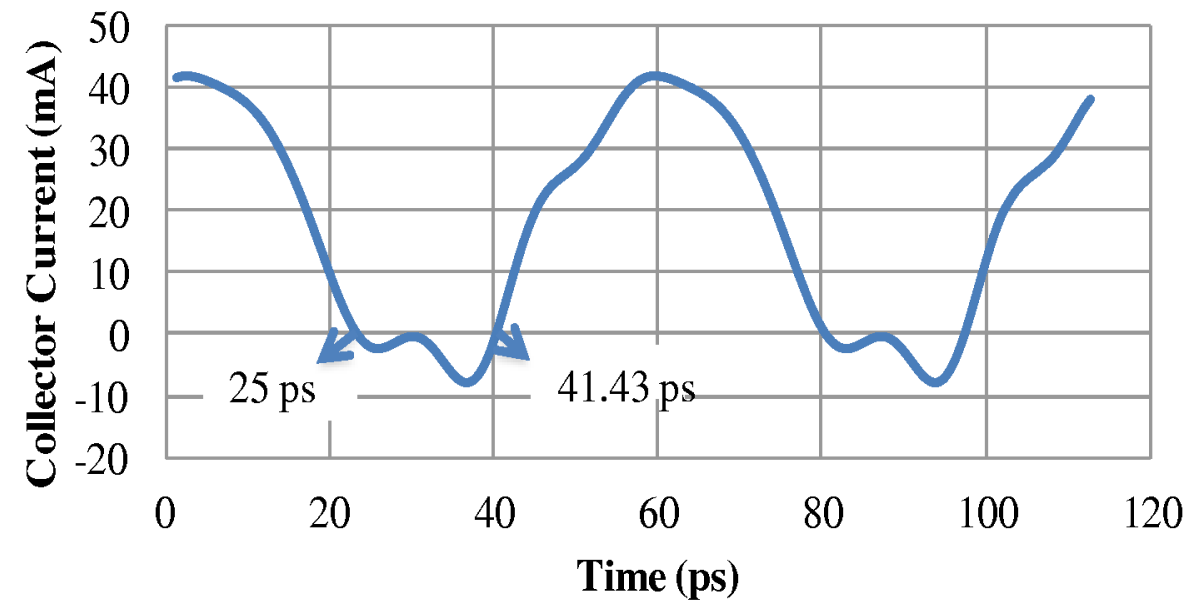

Figure 5.15 Collector current of the last stage

From this figure, the duration of time where the transistor is "OFF" is between $25 \mathrm{ps}$ and 41.43 ps. Based on (5.1) and knowing the period of the signal, we can calculate the conduction angle of the amplifier [10].

$$
\theta=\left(1-\frac{t_{\text {off }}}{T}\right) \times 360^{\circ}
$$


Figure 5.15 and (5.1) show that at $17.5 \mathrm{GHz}$, which is the centre frequency of the RTWO's tuning range, the conduction angle is $256.5^{\circ}$ which is closer to class B power amplification than class A [57].

In general, stability is a challenging task in a power amplifier design. In our case of having a three-stage power amplifier with high amount of gain, making the design stable is even more challenging especially after including the effect of parasitic inductances of the wire-bonds in the simulations.

As seen in Figure 5.14, an $R C$ network has been employed to decouple the $V_{C C}$ of all three stages in order to improve the stability. The small $14.5 \Omega$ resistor in series with the $25 \mathrm{pF}$ capacitor is used to dampen the $Q$ factor of the capacitor and improve the stability.

The $2 \mathrm{pF}$ capacitor in parallel with this network is used to decouple the unwanted high frequency signals. All DC pads are ESD protected and the bases of the transistors are biased with on-chip voltage regulators decoupled with the same on-chip $R C$ network. Since the PA is realized with a coplanar structure with a large distributed ground plane, EM co-simulation was used to verify the performance and, more importantly, the stability of the amplifier.

A parallel $R C$ circuit was used to model the substrate connection of the transistors in our EM co-simulations using Keysight's Momentum. This $R C$ modeling is used because we did not have access to the substrate contact, ptap, model of the design kit in our Momentum substrate definition. A parallel combination of a $10 \mathrm{~K} \Omega$ resistor and a $20 \mathrm{fF}$ capacitor has been used for this purpose. 
Full EM simulations of the PA predicted some instability at lower frequencies. Therefore two small series resistors, $10 \Omega$, have been added, encircled in Figure 5.14, to the output of the first and second stages to alleviate the problem.

\subsubsection{EM Simulation Results of the Power Amplifier}

Figure 5.16 shows the small-signal parameters of the full PA using Momentum tool. From this figure the peak gain of $27.5 \mathrm{~dB}$ and return loss better than $7.5 \mathrm{~dB}$ are achievable over the RTWO's tuning range from $17 \mathrm{GHz}$ to $18.2 \mathrm{GHz}$.

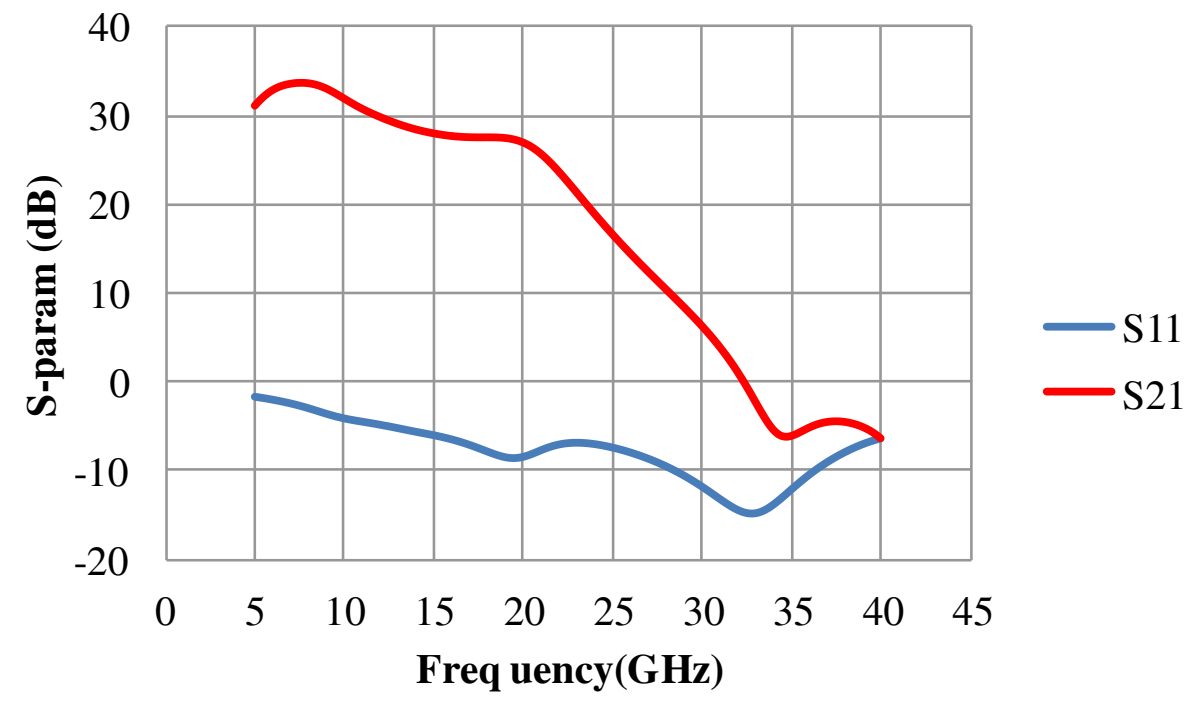

Figure 5.16 Full EM simulation results of the PA

Figure 5.17 shows the EM-simulated 1-dB compression point, gain and saturated power of the amplifier using Harmonic Balance simulation.

From this figure, the output referred $1-\mathrm{dB}$ compression point is $+2.5 \mathrm{dBm}$ with maximum saturated power of $+9 \mathrm{dBm}$. 


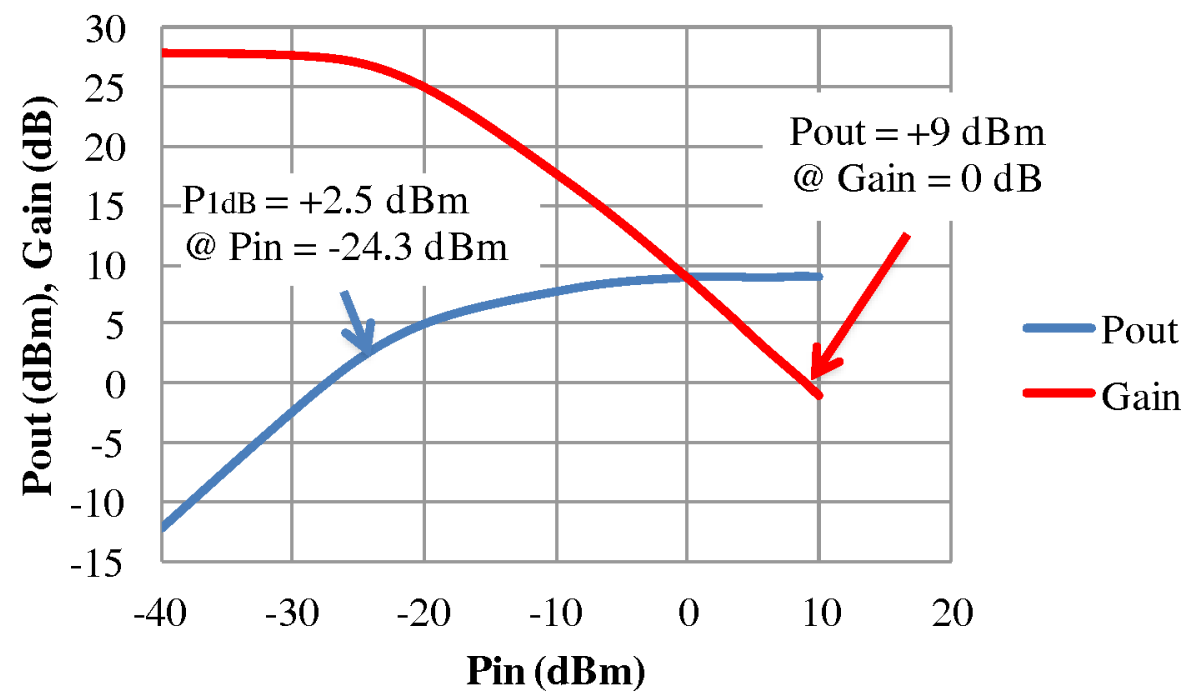

Figure 5.17 EM simulated results of the PA showing 1dB compression point and saturated power

Efficiency, $\eta$, is the measure of how effectively power from the supply is converted into output power and is given by (5.2), [58]:

$$
\eta=\frac{P_{\text {out }}}{P_{d c}}
$$

where $P_{\text {out }}$ is the RF output power.

Power added efficiency (PAE) is similar to efficiency, but it takes into account the RF power that is provided to the device at its input, in the numerator. PAE is the mostaccepted FOM to use to compare single devices and can be calculated based on (5.3).

$$
\text { P.A.E }=\frac{P_{\text {out }}-P_{\text {in }}}{P_{d c}}
$$


Figure 5.18 shows the EM-simulated maximum PAE of our PA. For this figure the maximum PAE of the PA is $5.8 \%$ which should be almost the same as $\eta$ in our case due to the high small signal gain of the design.

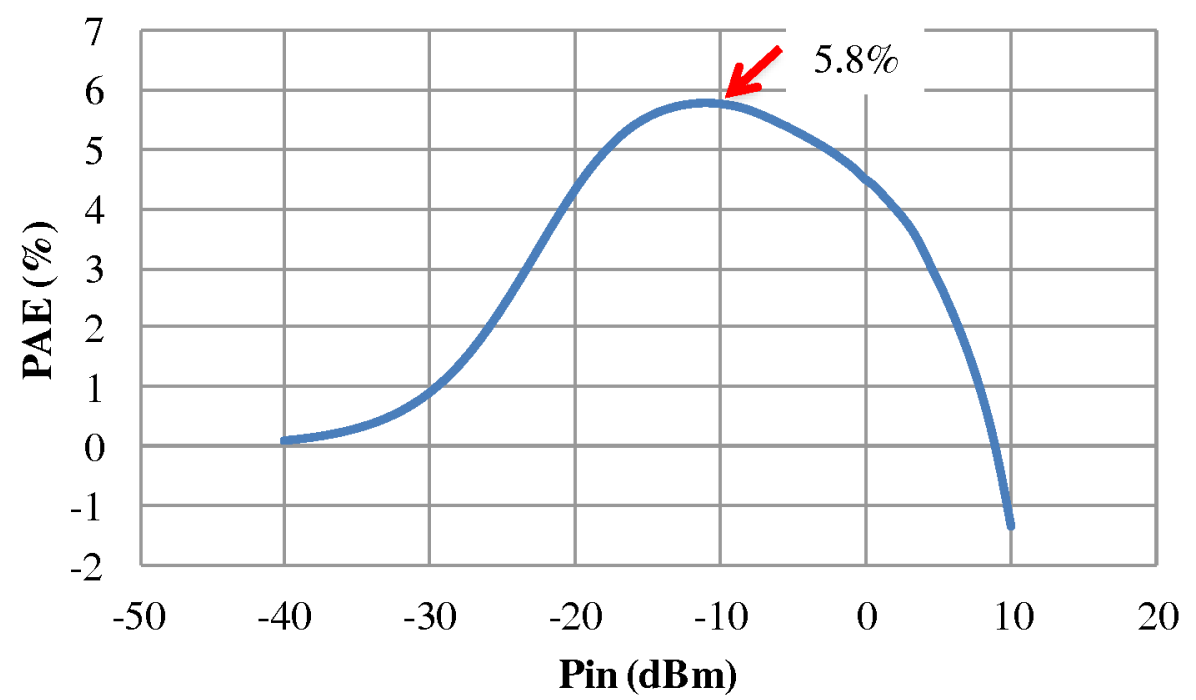

Figure 5.18 EM simulated PAE of the PA

Figure 5.19 shows the EM-simulated stability factor of the PA. In order to have an unconditionally stable amplifier, the stability factor $m u$, calculated by the ADS simulator, should be greater than one. We have simulated the circuit form $500 \mathrm{MHz}$ to $100 \mathrm{GHz}$ and as can be seen from this figure, this factor is greater than one across the whole range. 


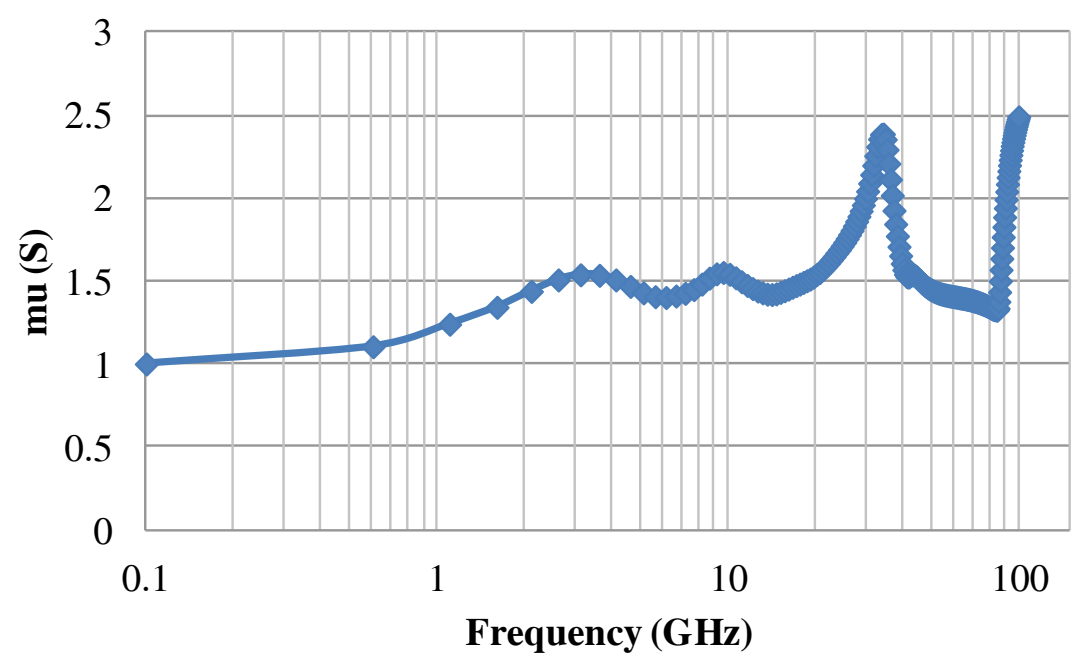

Figure 5.19 EM simulated stability factor of the amplifier showing the amplifier is unconditionally stable

\subsubsection{Characterization of the PA}

In order to characterize the PA, we have designed a stand-alone circuit and the following measurement results are obtained from on-wafer measurement. Figure 5.20 shows the die photo-micrograph of the PA realized in the same technology as the RTWO, distribution network and switches. The PA's total size, including the pads, is $1.7 \mathrm{~mm}^{2}$. We have considered an individual pad for DC bias of every collector and base nodes.

Figure 5.21 shows the measured small-signal parameters of the PA. From this figure the small-signal gain of the PA is $25 \mathrm{~dB}$ with $S_{11}$ better than $-8 \mathrm{~dB}$ from $16-20 \mathrm{GHz}$ which is much wider than the tuning range of the RTWO. 
All measured results are in agreement with our EM simulated results. Our measured gain is $2.5 \mathrm{~dB}$ lower than our simulated result which appears to be due to EM modeling of the inductor substrate shielding.

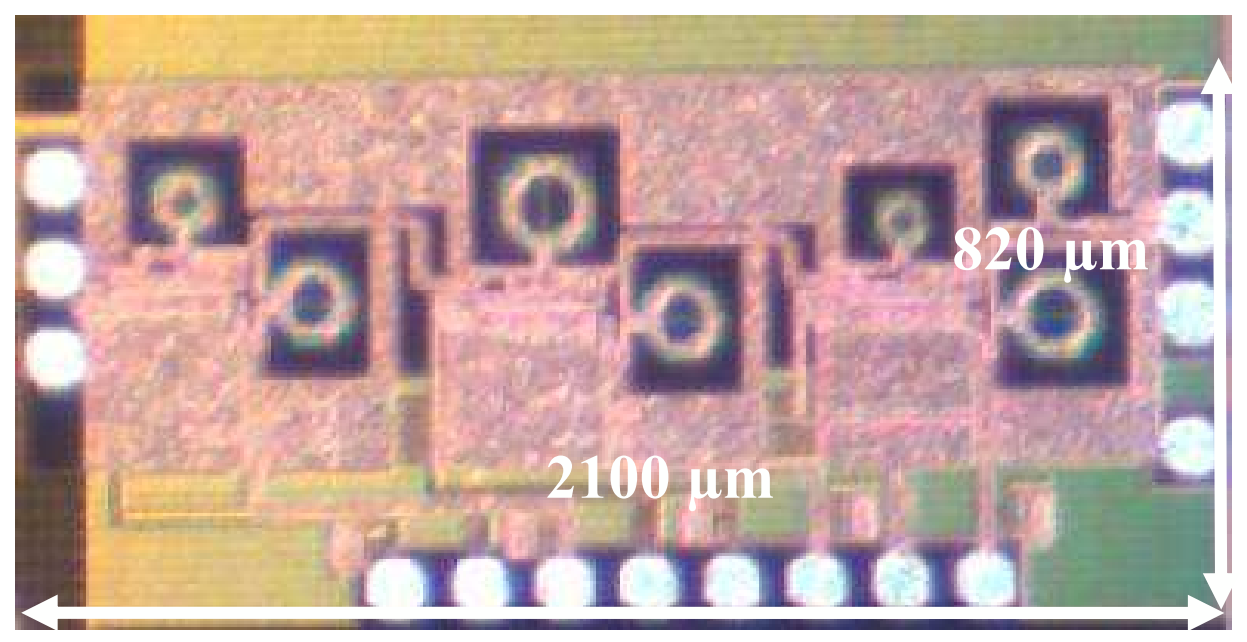

Figure 5.20 Die photomicrograph of the PA

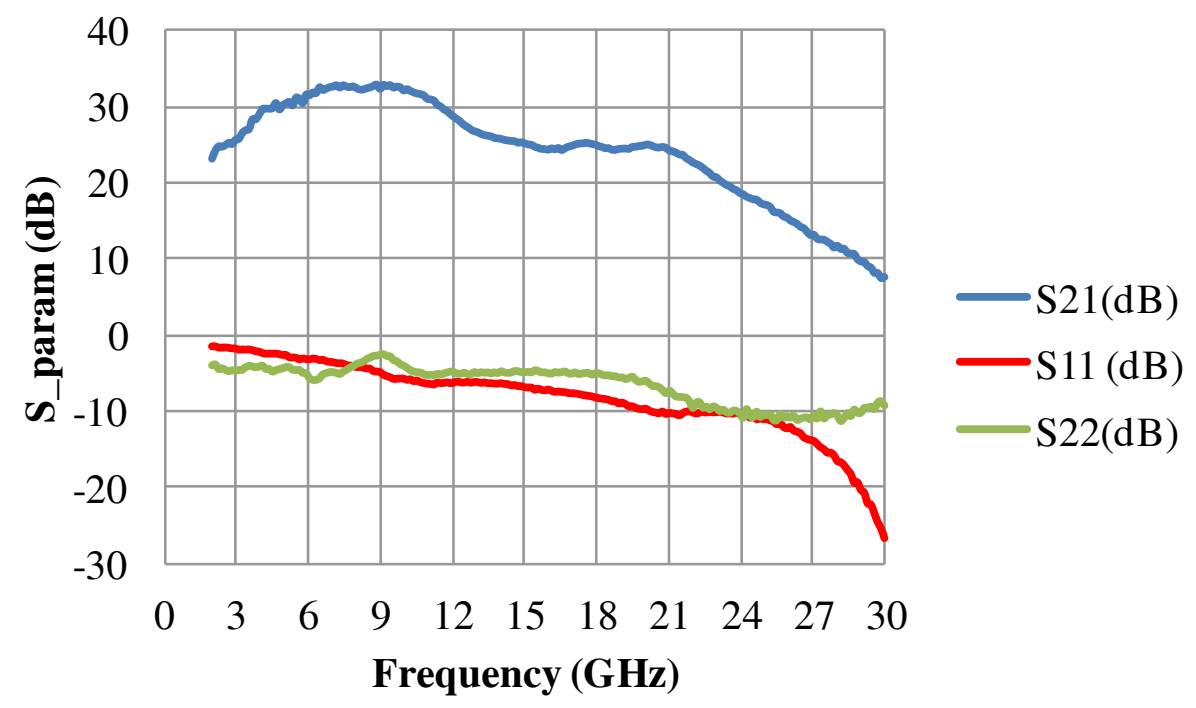

Figure 5.21 Measured small-signal parameters of the PA 
Figure 5.22 shows the measured output power of the PA. As can be seen in this figure, the PA output power at its $1 \mathrm{~dB}$ compression point is $+2 \mathrm{dBm}$, for an input power of $-22 \mathrm{dBm}$. The gain of the PA is quite high and therefore the amplifier saturates at low input powers. The measured saturated output power of the $\mathrm{PA}$ is $+7.5 \mathrm{dBm}$.

Measurement results shown in Figure 5.23 confirm that the PA is unconditionally stable. In order to measure the small signal parameters of the PA we have used a $40 \mathrm{GHz}$ HP 8722ES Vector Network Analyzer and that is why the value of measured $m u$ is constant after $40 \mathrm{GHz}$ because there is no data available beyond this frequency.

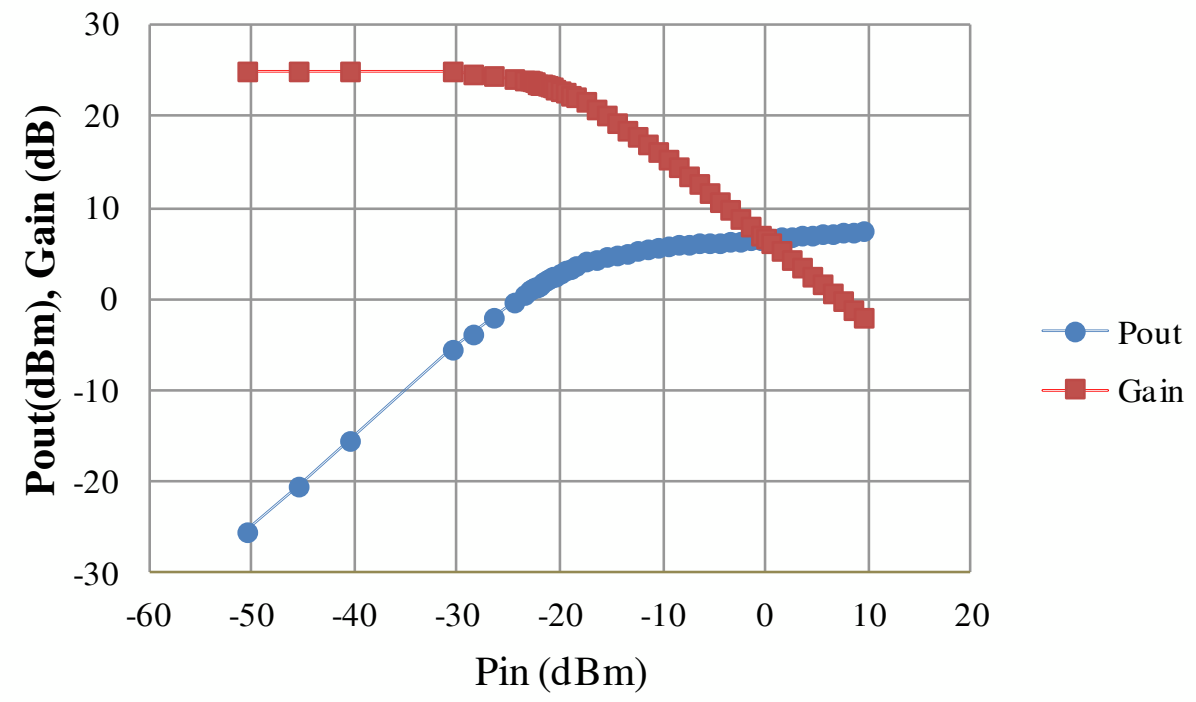

Figure 5.22 Measured power performance of the PA 


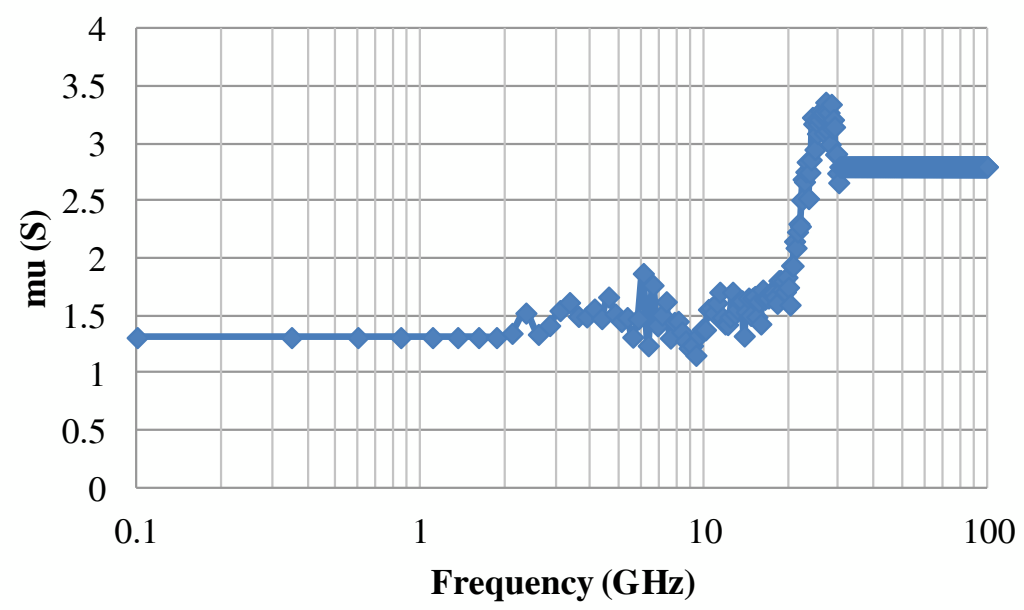

Figure 5.23 Calculated stability factor obtained from measured S-parameters

Table 4 shows the performance summary of the PA, and compares it to previously published designs.

From this table, the simulated PA results are in close agreement with measured results thanks to the EM co-simulation of the full circuit and $R C$ modeling of the substrate connections.

Comparing our PA with other published designs we can see that the small-signal gain of our PA is higher than others which can be quite useful for avoiding many amplification stages in the same die for phased-array system design. 
Table 4 Performance summary of the PA

\begin{tabular}{|c|c|c|c|c|c|}
\hline & $\begin{array}{c}\text { Our } \\
\text { simulated } \\
\text { results }\end{array}$ & $\begin{array}{c}\text { Our } \\
\text { measured } \\
\text { results }\end{array}$ & {$[\mathbf{3 6}]$} & {$[\mathbf{5 6}]$} & {$[\mathbf{5 9}]$} \\
\hline Tech & $\begin{array}{c}\mathbf{0 . 2 5} \boldsymbol{\mu m} \\
\text { BiCMOS }\end{array}$ & $\begin{array}{c}\mathbf{0 . 2 5} \boldsymbol{\mu m} \\
\text { BiCMOS }\end{array}$ & $\begin{array}{c}\mathbf{0 . 1 8} \boldsymbol{\mu m} \\
\text { BiCMOS }\end{array}$ & $\begin{array}{c}\mathbf{0 . 1 8} \boldsymbol{\mu m} \\
\text { BiCMOS }\end{array}$ & $\begin{array}{c}\mathbf{0 . 1 2} \boldsymbol{\mu m} \\
\text { BiCMOS }\end{array}$ \\
\hline Freq. (GHz) & $\mathbf{1 8}$ & $\mathbf{1 8}$ & 24 & 24 & 77 \\
\hline $\mathbf{S}_{\mathbf{2 1}}(\mathbf{d B})$ & $\mathbf{2 7 . 5}$ & $\mathbf{2 5}$ & 7 & 7 & 17 \\
\hline Peak PAE & $\mathbf{5 . 8 \%}$ & $\mathbf{5 \%}$ & 6.5 & 6.5 & $12.8 \%$ \\
\hline P $_{1 \mathbf{1 d B}}(\mathbf{d B m})$ & $\mathbf{2 . 5}$ & $\mathbf{2}$ & 11 & 11 & 10.2 \\
\hline $\mathbf{P}_{\text {sat }}(\mathbf{d B m})$ & $\mathbf{9}$ & $\mathbf{7 . 5}$ & 14 & 14 & 17.5 \\
\hline $\begin{array}{c}\text { DC power } \\
\text { (mW) }\end{array}$ & $\mathbf{6 2}$ & $\mathbf{7 8}$ & 170 & 280 & 297 \\
\hline
\end{tabular}

However, the 1-dB compression point and saturated power of our PA is lower than other reported results. This is due to the fact that our PA has higher small signal gain which saturates the amplifier quickly. The power consumption of our design is lower than other designs, which is beneficial for phased-array applications where so many components are operating and generating heat at the same time.

It should be noted that for the monolithic integration of the system, the PAs should be wire-bonded on the printed circuit board. However, having on-wafer measurement results which are in close agreement with our simulation results, provide a good degree of confidence for the adaptation of the PAs in the wire-bond environment.

\subsection{Coplanar Patch Antenna}

In order to analyse the full capability of our phased-array transmitter, we have designed an off-chip antenna-array in order to take into account its behaviour and constraints. As explained in section 5.4, in order to limit the complexity of the system 
assembly we have employed a coplanar structure in the realization of the PA. The coplanar structure simplifies the connection of the die to the PCB because no RF vias through the substrate are needed to make a ground connection. This concept allows the most readily available connection between the silicon die and the antenna's feed lines on the printed circuit board.

We have chosen Rogers RO4003 material for the PCB and antenna design. This material has been chosen because of its low loss and small thickness. The main characteristics of this material and the metal thickness we have used for the antenna implementation are summarized in Table 5.

Table 5 Rogers RO4003 material characteristics

\begin{tabular}{|c|c|c|c|}
\hline $\boldsymbol{\varepsilon}_{\boldsymbol{r}}$ & H (height) & Loss Tangent & Metal, thickness \\
\hline 3.33 & $0.508 \mathrm{~mm}$ & 0.003 & Copper, $18 \mu \mathrm{m}$ \\
\hline
\end{tabular}

\subsubsection{Antenna Design}

Figure 5.24 shows the coplanar patch antenna we have employed in this design. We have followed the same procedure as $[\mathbf{6 0}]$ and [61] for the antenna design except we have modified their design for our particular resonance frequency and substrate material.

The wavelength of a signal on the microwave substrate is calculated based on (5.4), $[62]$.

$$
\lambda_{g}=\frac{c}{f_{r} \sqrt{\varepsilon_{e f f}}}
$$


where $c$ is the speed of the light, $f_{r}$ is the resonant frequency and $\varepsilon_{e f f}$ is the effective permittivity of the substrate.

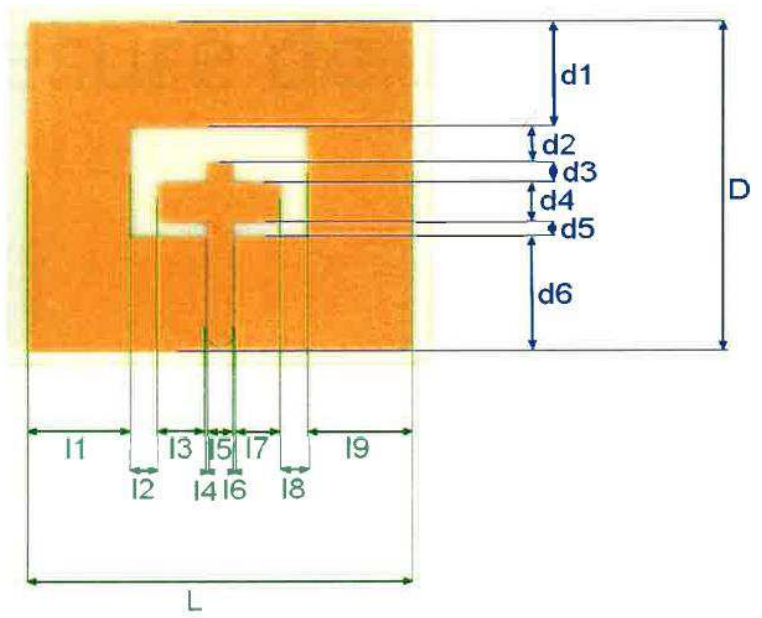

Figure 5.24 Coplanar antenna structure used in our design

The effective permittivity for a coplanar structure is approximated by:

$$
\varepsilon_{e f f}=\frac{\varepsilon_{r}+1}{2}
$$

In our case and for resonance frequency of $18 \mathrm{GHz}$ on RO4003 material, $\lambda_{g}$ is equal to $11.3 \mathrm{~mm}$ and $\varepsilon_{e f f}$ is equal to 2.165 .

The length of the patch can now be calculated using (5.6) [61]:

$$
L_{p}=\frac{c}{2 f_{r} \sqrt{\varepsilon_{e f f}}}
$$

Using the above calculated numbers for $f_{r}$ and $\varepsilon_{e f f}$, the length of the patch should be equal to $5.6 \mathrm{~mm}$. 
Table 6 summarizes the dimensions of the antenna shown in Figure 5.24, designed to operate in the Ku-band frequency.

Table 6 Antenna dimensions based on Figure 5.24

\begin{tabular}{|c|c|c|c|c|c|c|c|c|}
\hline L1 & L2 & L3 & L4 & L5 & D6 & D5 & D4 & D3 \\
\hline $4.4 \mathrm{~mm}$ & $1.2 \mathrm{~mm}$ & $1.8 \mathrm{~mm}$ & $0.15 \mathrm{~mm}$ & $1 \mathrm{~mm}$ & $5 \mathrm{~mm}$ & $0.7 \mathrm{~mm}$ & $1.7 \mathrm{~mm}$ & $0.9 \mathrm{~mm}$ \\
\hline
\end{tabular}

Figure 5.25 shows the EM simulation results of the antenna using HFSS. We have designed and simulated three different antennas with different dimensions, all close to the dimensions given in Table 6 with small changes, since we have to make sure that both the power amplifier and the antenna have a frequency bandwidth which can cover the RTWO's frequency range.

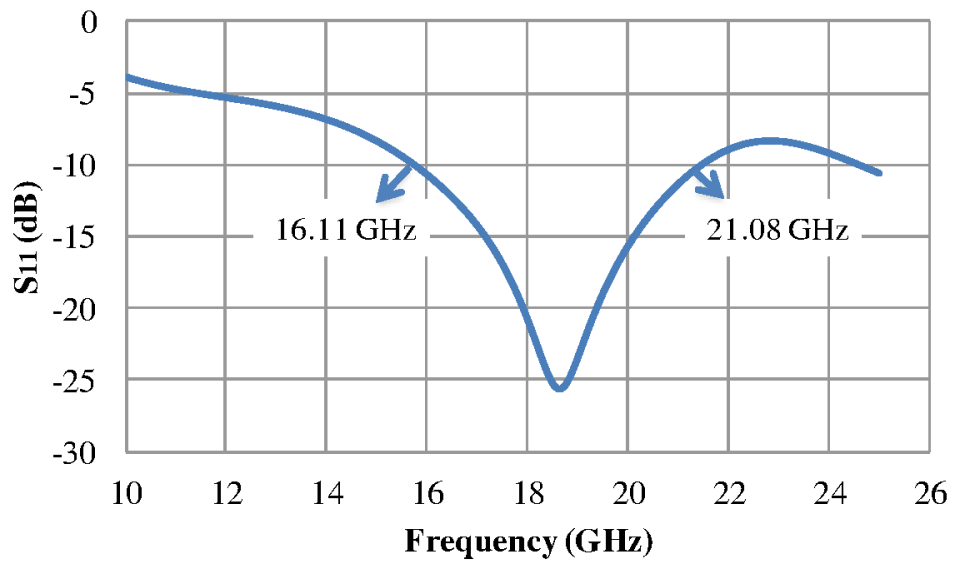

Figure 5.25 HFSS simulation results of the antenna showing the $-10 \mathrm{~dB}$ bandwidth

Figure 5.26 shows the simulated radiation pattern of the antenna shown in Figure 5.24. From this figure the antenna gain, in a far field setup, is $4.7 \mathrm{~dB}$. 

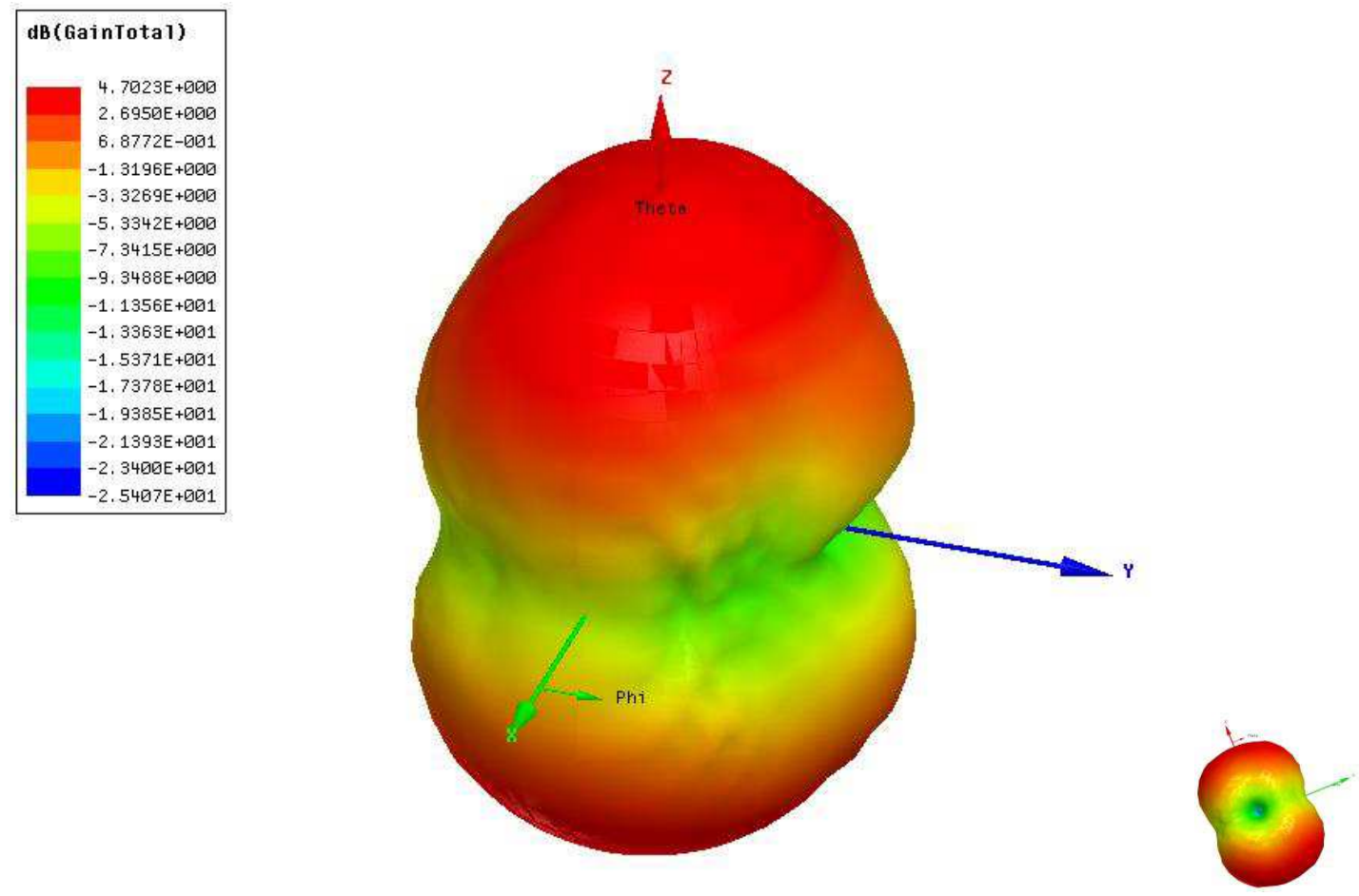

Figure 5.26

HFSS simulation results for the antenna radiation pattern

In the following sub-section we show the measurement results of the antenna fabricated using the dimensions in Table 6 .

\subsubsection{Antenna Characterization}

Figure 5.27 shows a photograph of the antenna both alone and in close proximity with the second identical antenna.

We have characterized the antenna separately and also in close proximity with the second similar antenna in order to evaluate the coupling effect between them. This has been done because for the implementation of the two-element array transmitter, two antennas will be fabricated close to each other and on the same PCB for beam-forming. 


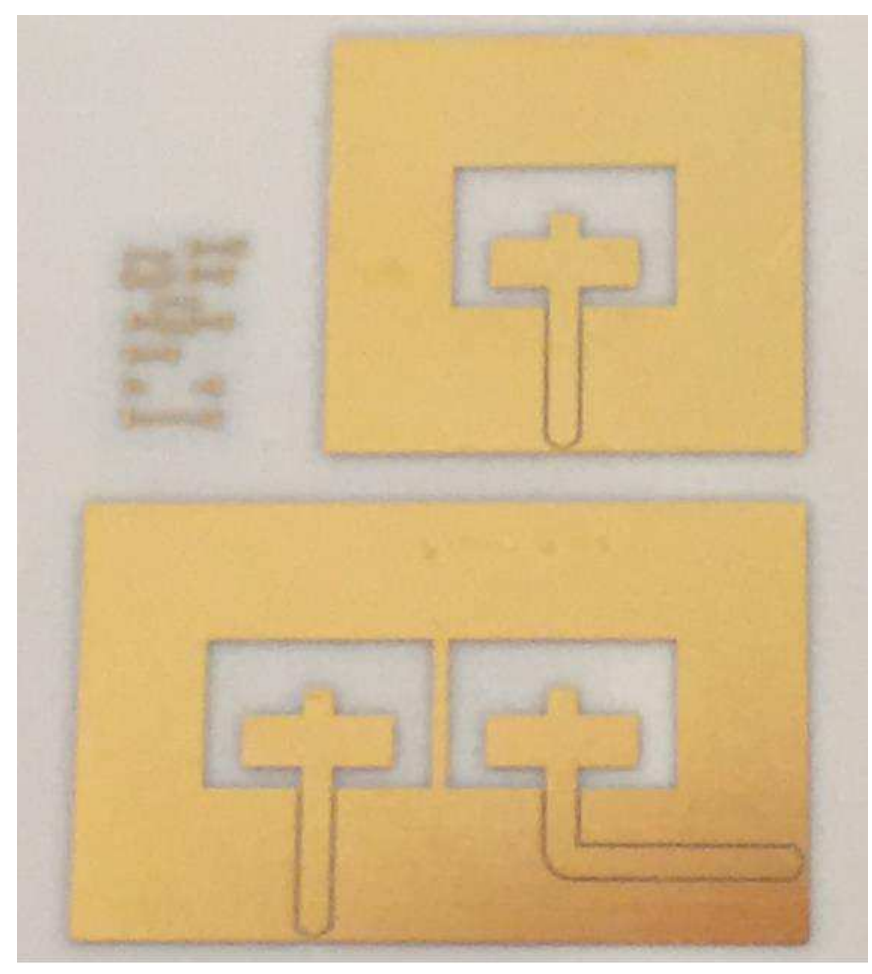

Figure 5.27 Photograph of the antenna and two antennas with an $8 \mathrm{~mm}$ separation

Ideally, the antenna elements in an antenna array are placed half a wavelength apart which corresponds to $8.34 \mathrm{~mm}$ at $18 \mathrm{GHz}$. However, because of the presence of a ground plane around the active radiating element, this distance is $8 \mathrm{~mm}$ in our case. This value corresponds to 0.48 times of the free space wavelength and the radiation angle can be calculated based on (5.7).

$$
\theta=\sin ^{-1}\left(\frac{\varphi \lambda}{2 \pi d}\right)=\sin ^{-1}\left(\frac{\varphi}{0.96 \pi}\right)
$$

Measurement of the antenna was performed on-chip using high frequency GSG probes and a high frequency vector network analyzer. Our designed antenna is supposed to radiate in both $\pm Z$ directions and we have not added any ground plane under the 
antenna in our HFSS simulations. As a result, during the measurement procedure, very careful attention has to be paid to keep the antenna as far as possible from the probe station which can behave as a ground plane under the antenna.

However, we have estimated the presence of a ground plane at $5 \mathrm{~mm}$ away from the substrate and we have added a ground plane at $5 \mathrm{~mm}$ below the substrate in our modified simulation to be able to match the measurement results.

Figure 5.28 shows the measurement results of the antenna for different distances from the probe station. Distance 1 represents the thickness of a sheet of paper which is used to elevate the antenna from the probe station's chuck, distance 2 is the thickness of a plastic interposer, $2.5 \mathrm{~mm}$, used to elevate the antenna from the probe's chuck and distance 3 is the thickness of two plastic cards, $5 \mathrm{~mm}$, from the probe station's chuck. As can be seen from this figure, distance 3 gives the best results as expected.

Figure 5.29 shows the measured coupling effect between the two identical antennas being apart from each other by $8 \mathrm{~mm}$. In order to do this measurement, we have load the second antenna by $50 \Omega$ as well. Doing this, $\mathrm{S}_{12}$ parameters should give the indication of the coupling between the two antennas.

We can see a good agreement between simulation and measurement results of the antenna given in Table 6 and we are hoping to get the same agreement between the simulations and measurement results of the other two fabricated antennas. 


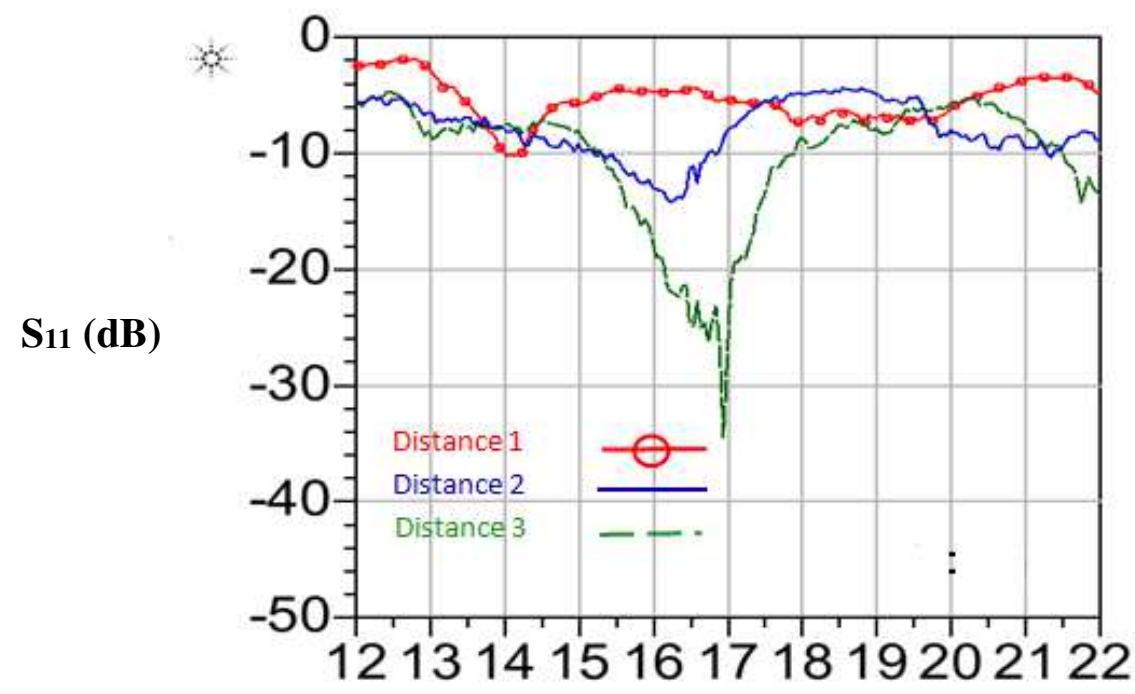

Frequency (GHz)

Figure 5.28 Measured $S_{11}$ results for the antenna shown in Figure 5.24 for three different distances between the antenna and the probe chuck. Distance 1 is equal to the thickness of a sheet of paper, Distance 2 is equal to $2.5 \mathrm{~mm}$, and Distance 3 is equal to $5 \mathrm{~mm}$

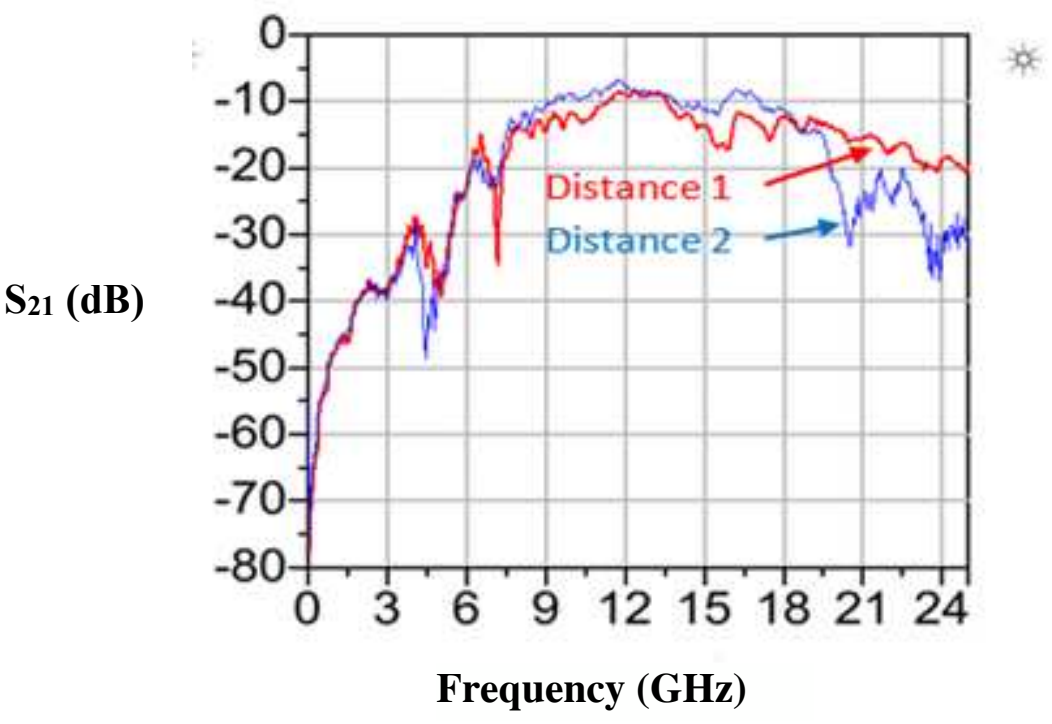

Figure 5.29 Measured $S_{12}$ results for the two antennas for two different distances between the antenna and the probe chuck. Distance 1 is equal to $2.5 \mathrm{~mm}$ and Distance 2 is equal to $5 \mathrm{~mm}$ 


\subsection{Summary}

This chapter provides the design steps, layout considerations and also measurement results of different building blocks needed for design of a Ku-band two-element phasedarray transmitter.

A custom-designed distribution network is needed in our case since the outputs of the RTWO are at different positions on the resonator while all the outputs have to be distributed to the right side of the die, for a unidirectional phased-array structure, in order to feed the antenna array for beam-forming. The designed distribution network shows only $3^{\circ}$ of phase error between the arms but it consumes $35 \%$ of the full system layout area.

Our network might not be the best candidate for phased-array transmitters with many elements, because of its size, but it is still comparable both in size and performance with the conventional H-tree distribution network. However, size reductions of our distribution network, in both the $\mathrm{X}$ and $\mathrm{Y}$ directions, are possible. For size reduction in the $\mathrm{X}$ direction, we can take advantage of the slow wave propagation concept and design a periodic $L C$ network to shrink the physical length of the arms. According to [42], the slow wave factor, $\lambda_{0} / \lambda_{g}$, is around 4.5 for $20 \mu \mathrm{m}$ wide microstrip structure on a GaAs substrate.

We can make the line width smaller at the expense of slightly higher loss, and shrink the network in the $\mathrm{Y}$ direction as well. For example, dividing the width of each arm by two, can divide the size of the distribution network by two in the Y direction. 
In order to design high frequency switches to select the desired phase of the RTWO, after being distributed to the right side of the layout, we have applied a body-floating technique to the non-isolated NMOS devices available in NXP's design kit. Connecting the body of the transistor to a high impedance, instead of ground, can improve the power handling and isolation of a switch. In our case where we need to select the desired phase of the RTWO and prevent the other phases from reaching the antennas, good isolation is critical and this technique can be very beneficial.

Applying this technique, we have employed multiple rings of DTI and ptap layers around the transistors while using a virtual mask layer to get access to the substrate of the device and connect it to the desired impedance.

The switches are symmetrical with the same input and output impedances. For the design of a two-element-array transmitter, we need two of these switching banks. For each switching bank we have designed 16 switches, of which eight are dummies and triggered with the complementary gate voltage of the real switches. This has been done to keep the loading of the distribution network constant during the phase selection otherwise this loading difference can contribute a significant amount of phase error. The complementary voltage needed to trigger the dummy switches is created by the means of an on-chip inverter added to each switching bank.

For the remaining blocks of the transmitter, including the PA and antenna, we have used a coplanar structure due to the simplicity of making die to PCB connections. This 
also eliminates the need for RF via holes through the substrate which are needed in microstrip structures.

We have designed a three stage class AB power amplifier for this design. The PA's first two stages are designed for high voltage gain and the last stage is designed for power handling. In order to stabilize the PA (with its high small-signal gain), we have used an $R C$ circuit in the power supply decoupling network.

Another $R C$ circuit is also used to model the substrate connection of the transistors in the PA's EM simulation setup. This substrate modeling $R C$ network is used in all of our simulations because we cannot model the substrate contact, ptap, in our Momentum substrate definition.

Comparing our PA to other published designs, we see that our designed PA shows lower output power than others while it achieves high gain with low power consumption. However, as explained in this chapter, we have designed the PA with high gain in order to compensate for the loss of the distribution network and the switching bank and to provide at least $+2 \mathrm{dBm}$ of output power to the antennas.

Otherwise, the EIRP of the two antennas in our two-element array transmitter should be equal to $20 \log 2+2 \mathrm{dBm}=8 \mathrm{dBm}$ at $1-\mathrm{dB}$ compression point of the $\mathrm{PA}$, which is more than enough to show the proof of the concept.

The off-chip patch antenna is behaving very similar to its HFSS simulation results and we have fabricated two antennas with $8 \mathrm{~mm}$ of spacing between them on the same PCB to measure the functionality of the fully integrated phased-array transmitter. 
The measurement of the antenna has been done for three different distances from the PCB to the probe chuck and out of which the highest distance between the antenna and the probe chuck gives the best results. This effect was expected because we have employed a CPW structure and not a CPWG structure for the design of the antenna and our measurement results agree with our CPW simulation results.

Simulation and measurement results of the full system are provided in the following chapter. 


\section{Chapter 6 Fully Integrated Two-}

\section{Element Array Transmitter Utilizing}

\section{an RTWO}

As discussed in Chapter 5, a two-element array transmitter is useful for beam-forming as we can radiate in the desired direction and increase the gain and efficiency of the system. In this chapter, the assembly of the silicon building blocks as a monolithic system and the connection to antennas on a printed circuit board are discussed. The measurement results of the full system are provided at the end of this chapter.

Designing an on-chip system including so many building blocks at high frequencies is very challenging especially if the final die needs to be wire-bonded on a PCB for measurement since the effect of the on-chip to off-chip transition should be taken into account.

Below is a list of challenges and difficulties which were encountered during the layout implementation of the two-element array transmitter as well as our proposed solutions.

1. The reference node of any die designed to be mounted on a PCB is not well defined. This is due to the fact that the ideal ground node with zero potential, and 
no parasitic inductance, will be somewhere on the $\mathrm{PCB}$, coming from the measurement equipment, and not on the die anymore. Simulating the extracted view of different parts of the circuit, we have to consider this fact and to avoid using an ideal ground connection in simulation. Instead, a local node on the die should be considered the reference node and some parasitic inductance should be used to connect this local node to the ideal ground node in all simulations.

2. The substrate connection of the components in silicon-based technologies is also critical and challenging for the explained case of a die which has to be bonded on a PCB. This connection has to be carefully defined with the layer of active layer in the Momentum substrate definition or if this layer is not available, like in our case, a parallel combination of $R C$ network has to be used for the substrate connection modeling of the critical components.

3. The effects of wire-bonds have to be taken into account at every stage of the design. We have evaluated the effect of $0.2 \mathrm{nH}-0.5 \mathrm{nH}$ inductance connected to each high frequency pad, which are only the outputs of the PA to the off-chip antennas, and also all ground pads of the circuit. Using more ground pads, by sacrificing more area, can ease the wire-bonding problem.

4. The PA tended to become unstable in the RTWO's frequency range for any grounding parasitic inductance higher than $0.1 \mathrm{nH}$. To fix this problem, we included ten ground pads for each PA layout and we also decoupled the supply voltages of the PA, all collector and base supply voltages, with an $R C$ network instead of a big decoupling capacitor. This $R C$ network significantly improved the 
stability of the circuit. The same $R C$ network has been used to decouple the RTWO's DC pads as well.

5. The PA is designed and matched for a $50 \Omega$ source and $50 \Omega$ load. From the output, the PA will be loaded by an off-chip coplanar antenna which has to be designed for a $50 \Omega$ impedance on the PCB. However, the input of the PA will be connected to the output of the switching bank and a matching network is required between these two components.

The PA is implemented using a coplanar structure and the distribution network and the switching bank are laid out using microstrip structure. As a result, the effect of the big vias needed for this transition from microstrip to coplanar has to be simulated using Momentum. Simulating such a large circuit with many vias, even after merging them, can cause convergence problems, due to the complexity of the circuit for the simulator, and the circuit has to be broken down into multiple smaller circuits. Doing this, we have to make sure that we take into account the effect of coupling between the smaller circuits when we cascade them to build the full circuit.

To summarize, a careful IC-PCB co-design methodology is mandatory to be able to accurately predict the electrical performance of such a big monolithic system. The lessons learned can also be applied for high performance and high frequency circuits which have to be packaged. 


\subsection{Monolithic Two-Element Array Transmitter}

Figure 6.1 shows the schematic diagram of the full two-element array transmitter utilizing an RTWO.

As can be seen from this figure, the outputs of the RTWO are delivered to the switching bank through a symmetric distribution network. The RTWO is biased and tuned with three DC pads to bias the core VCO, the buffers and the varactors. The total power consumption of the RTWO, including the eight buffers, is $703.5 \mathrm{~mW}$. We have used a $4.5 \mathrm{~V}$ source to bias the core $\mathrm{VCO}$ while the RTWO is also functional with a $2.5 \mathrm{~V}$ source and this can reduce the power of this block to $613 \mathrm{~mW}$.

The switching bank consists of 16 switches of which eight are real switches and the other eight are dummy switches for symmetric loading purposes as explained in Chapter 5. After selecting the desired phase of the RTWO, the outputs of the switching banks are combined through an eight-to-one combiner, as shown in Figure 6.1, and the output of the combiner will be the input of the power amplifier.

We have assigned one DC pad for each switching bank which is used to bias the onchip inverter and the role of the on-chip inverter is to provide the complementary voltage both to the gate of the shunt transistor in the real switch and also the gate of the series transistor in the dummy set. The control lines shown in this figure are connected to the gates of the series transistors in the real set of switches which will be connected to offchip switches for phase selection in the final application. 
The last components in Figure 6.1 are the top and bottom power amplifiers. Each power amplifier has six individual DC pads for base and collector biasing of all three stages and it consumes $90 \mathrm{~mW}$ of power from $2.5 \mathrm{~V}$ source which corresponds to $180 \mathrm{~mW}$ for two power amplifiers.

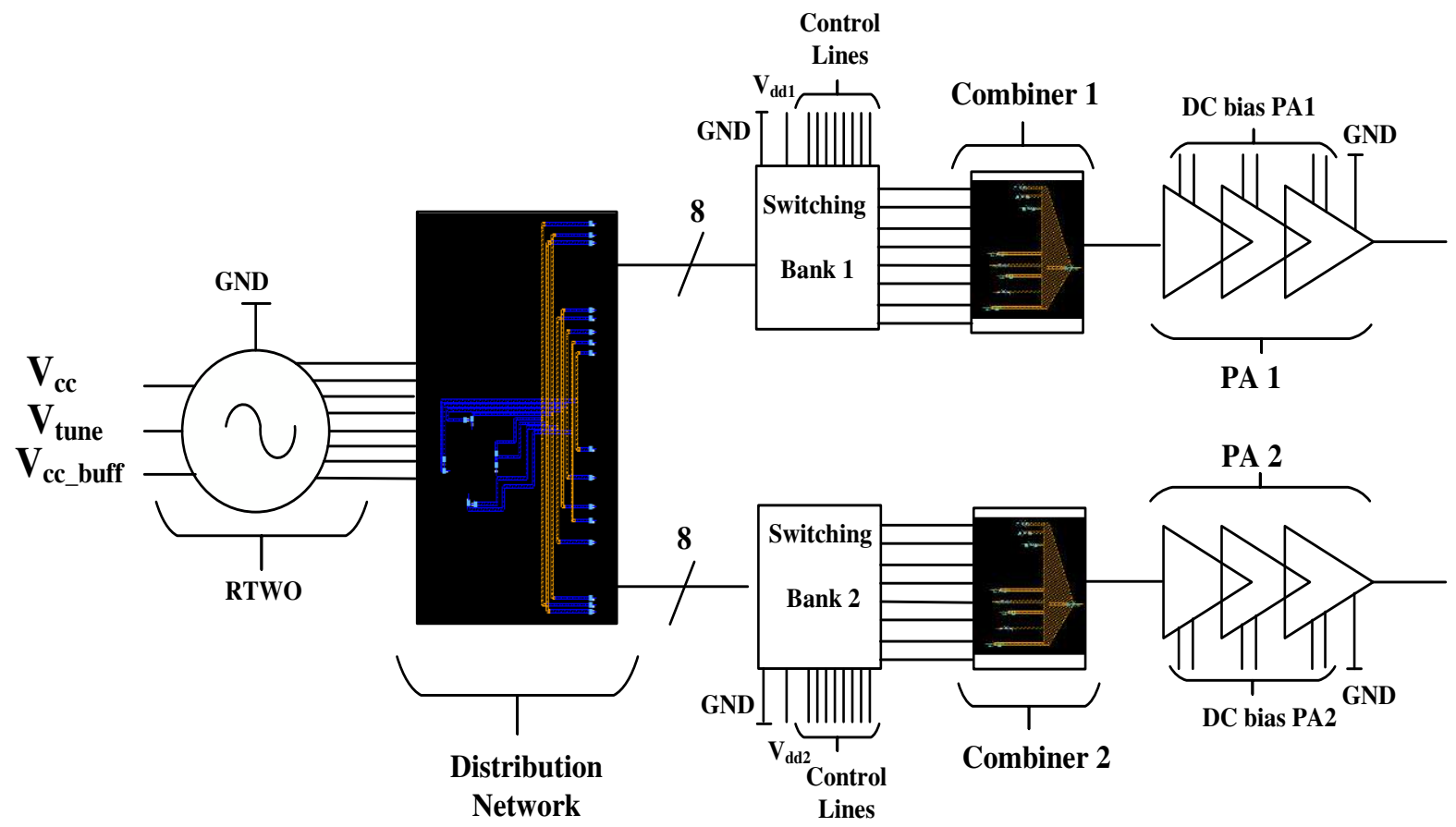

Figure 6.1 Schematic diagram of the full system

Figure 6.2 shows the die photo micrograph of the full two-element array transmitter fabricated in a $0.25 \mu \mathrm{m}$ BiCMOS process from NXP Semiconductors. The total size of the die is $19 \mathrm{~mm}^{2}$.

The RTWO previously discussed can be seen on the left side, whose outputs are distributed by the H-tree in the centre of the die (which consumes $35 \%$ of the chip area). The H-tree distributes the signal to a set of real and dummy switches which control the 
phases of the signals to be eventually sent to the antennas. Power amplifiers are seen on the top and bottom of the right side of the photo, which serve to compensate for the loss of the switches and distribution network and provide additional power to the antennas.

The total area of the RTWO is $0.57 \mathrm{~mm}^{2}$ and the total area of each PA is $1.7 \mathrm{~mm}^{2}$. The third PA, between the top and bottom PA, seen in this photo is a stand-alone PA used for probing and the measurement results of it are shown in Chapter 5. There is also a stand-alone RTWO seen on the left top corner of this graph which has also been used for probing. For this RTWO all segments are terminated to the loading impedance of the distribution network instead of $50 \Omega$.

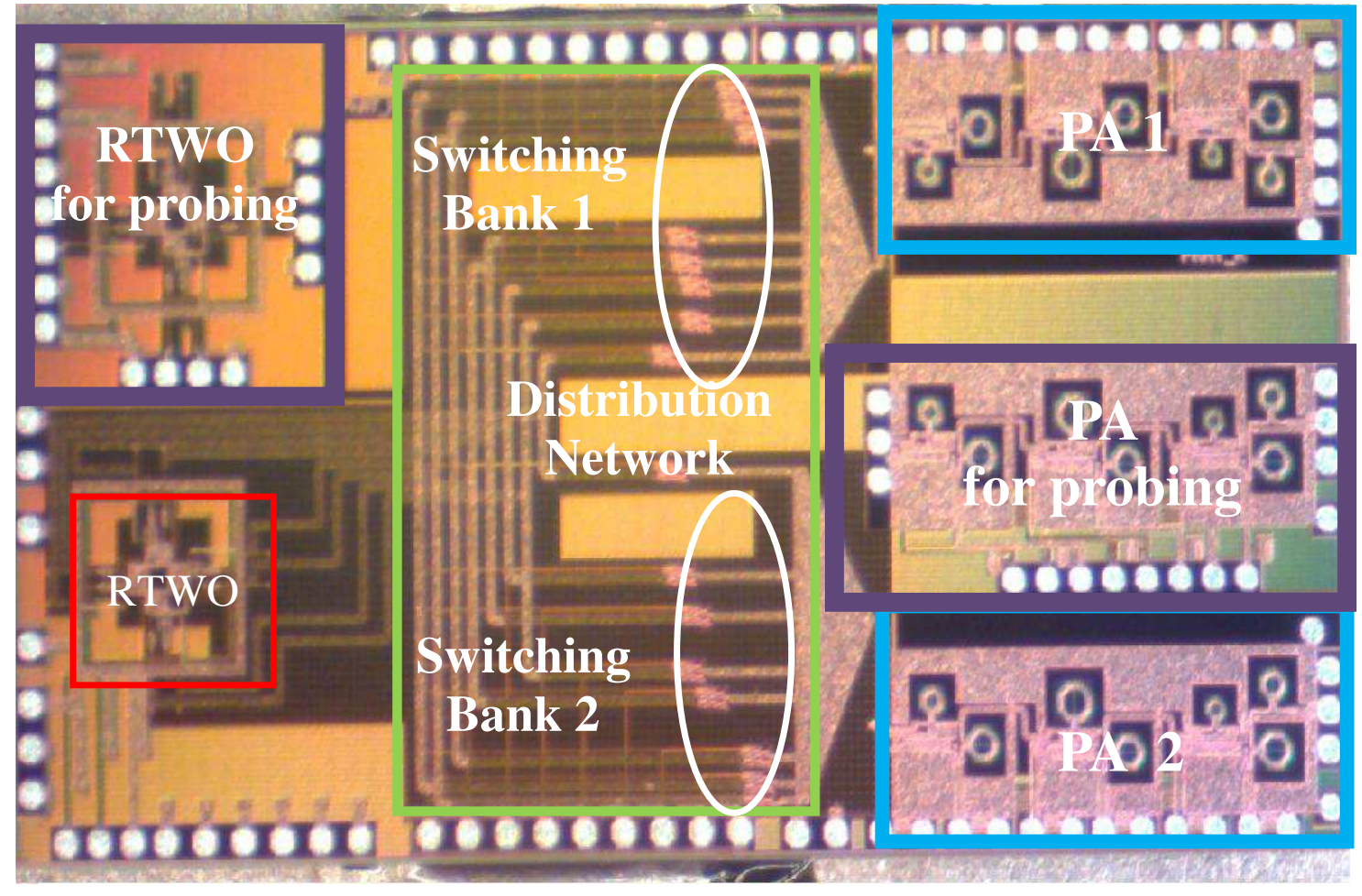

Figure 6.2 Die photo-micrograph of the two-element array transmitter 
Figure 6.3 - Figure 6.5 show the EM simulation results of the full system for RTWO phase shifts of $45^{\circ}, 90^{\circ}$ and $135^{\circ}$.

From these figures, we can see that the desired phase shifts are achieved at the output of the power amplifier. For a selected phase shift of $45^{\circ}$, the amplitudes of the two power amplifiers' outputs are $795 \mathrm{mV}$ and $785 \mathrm{mV}$. This amplitude mismatch is due to the mismatch of the distribution network, discussed in Chapter 5. The phase error is $2.25^{\circ}$, which is due to the RTWO phase error and additional error from the distribution network.

For the selected $90^{\circ}$ of phase shift, there is $2.65^{\circ}$ of phase error and finally for the selected $135^{\circ}$ of phase shift, the output voltages of the amplifiers are $749.5 \mathrm{mV}$ and $727 \mathrm{mV}$, with $5^{\circ}$ of phase error.

It should be noted that the same phase shift can be obtained for different switch inputs, which could provide better or worse phase error and amplitude imbalance.

The effect of $0.5 \mathrm{nH}$ inductance representing the wire-bond inductance connected to each ground pad of the system has been taken into account for all of our simulation results.

Due to the complexity of the full system the only simulation converged for the full circuit was transient analysis and in order to look at any other simulation the circuit has to be broken down at least to three smaller circuits. 

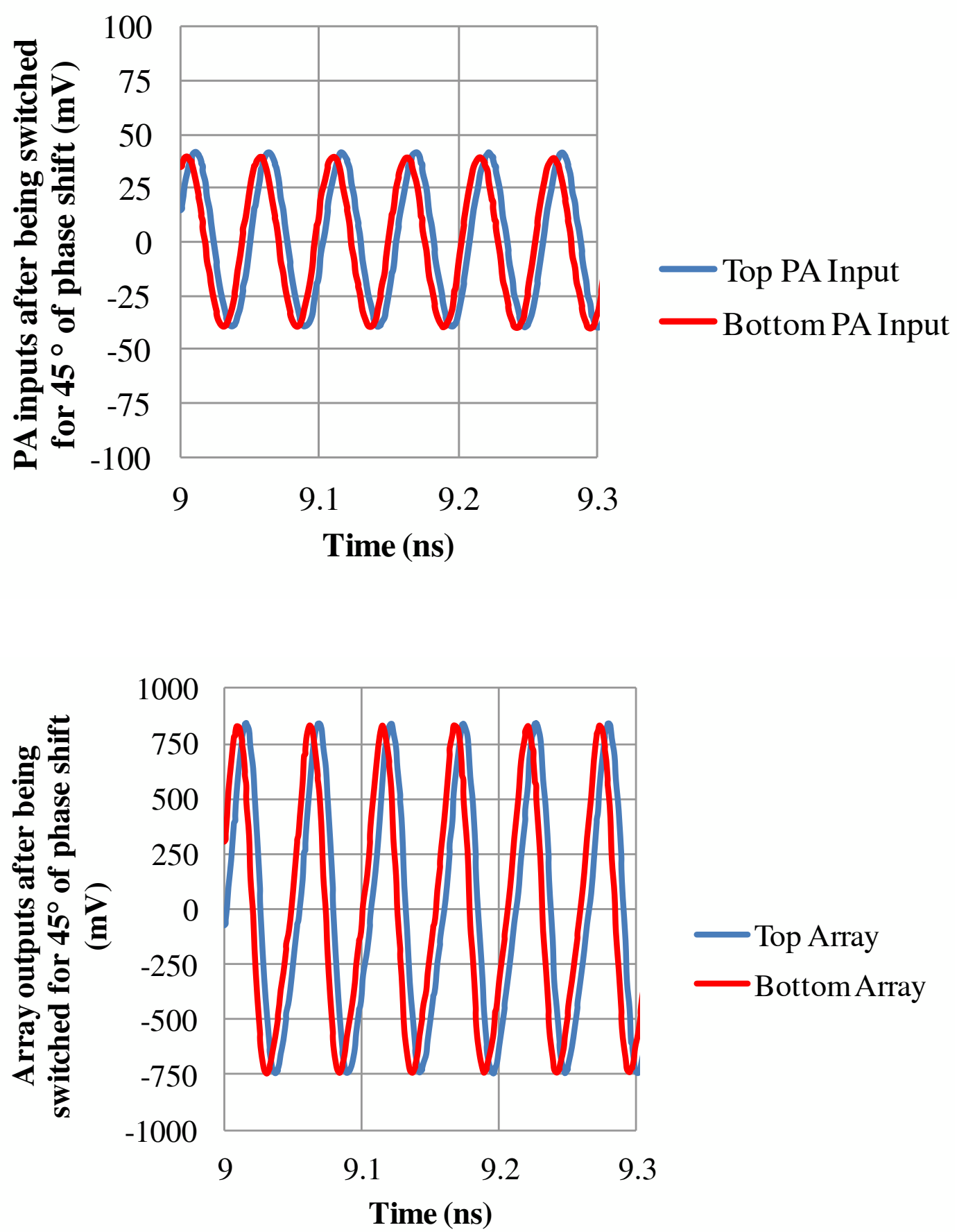

Figure 6.3 EM simulation results of the full system for RTWO's $45^{\circ}$ signals 

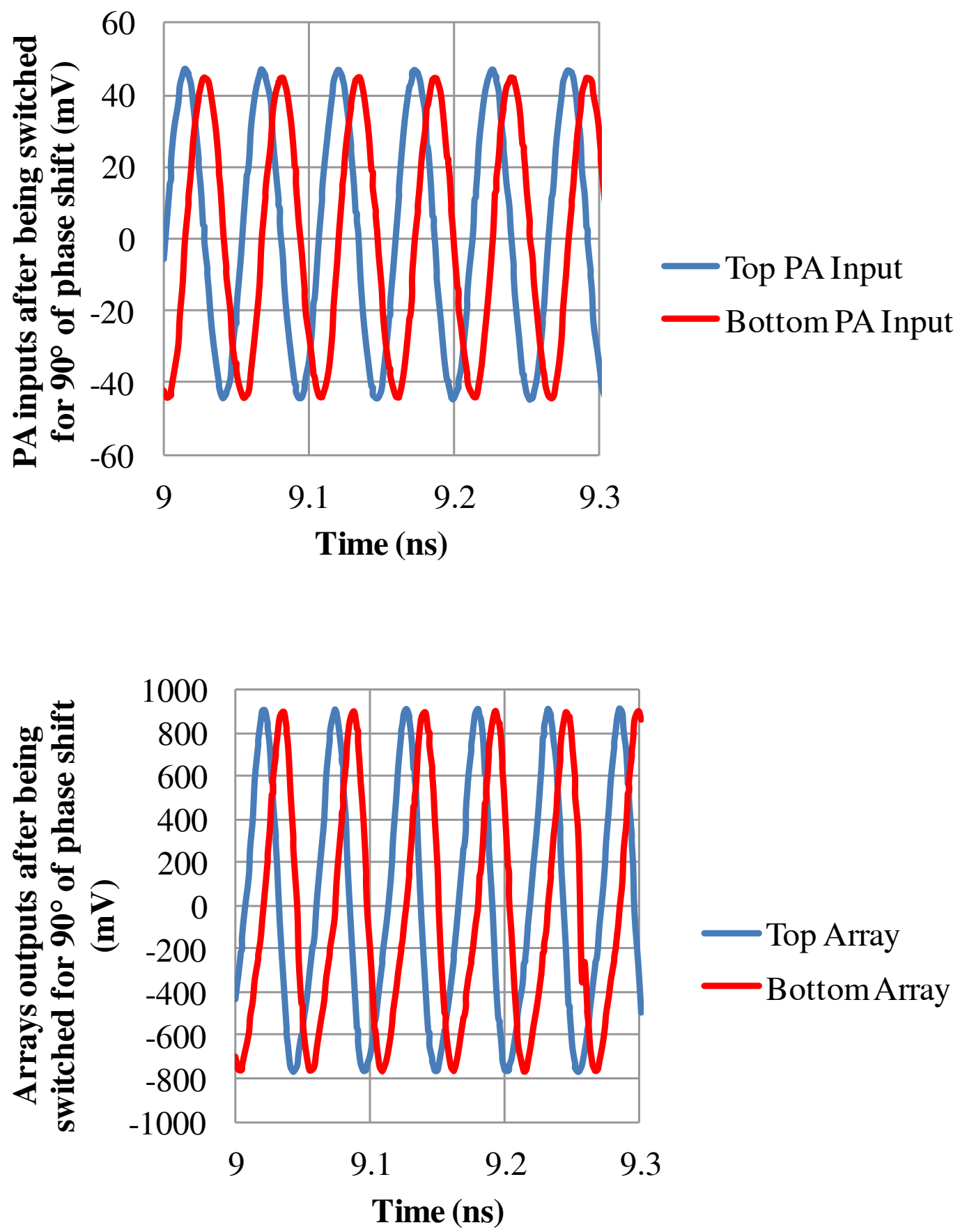

Figure 6.4 EM simulation results of the full system for the RTWO's $90^{\circ}$ signals 

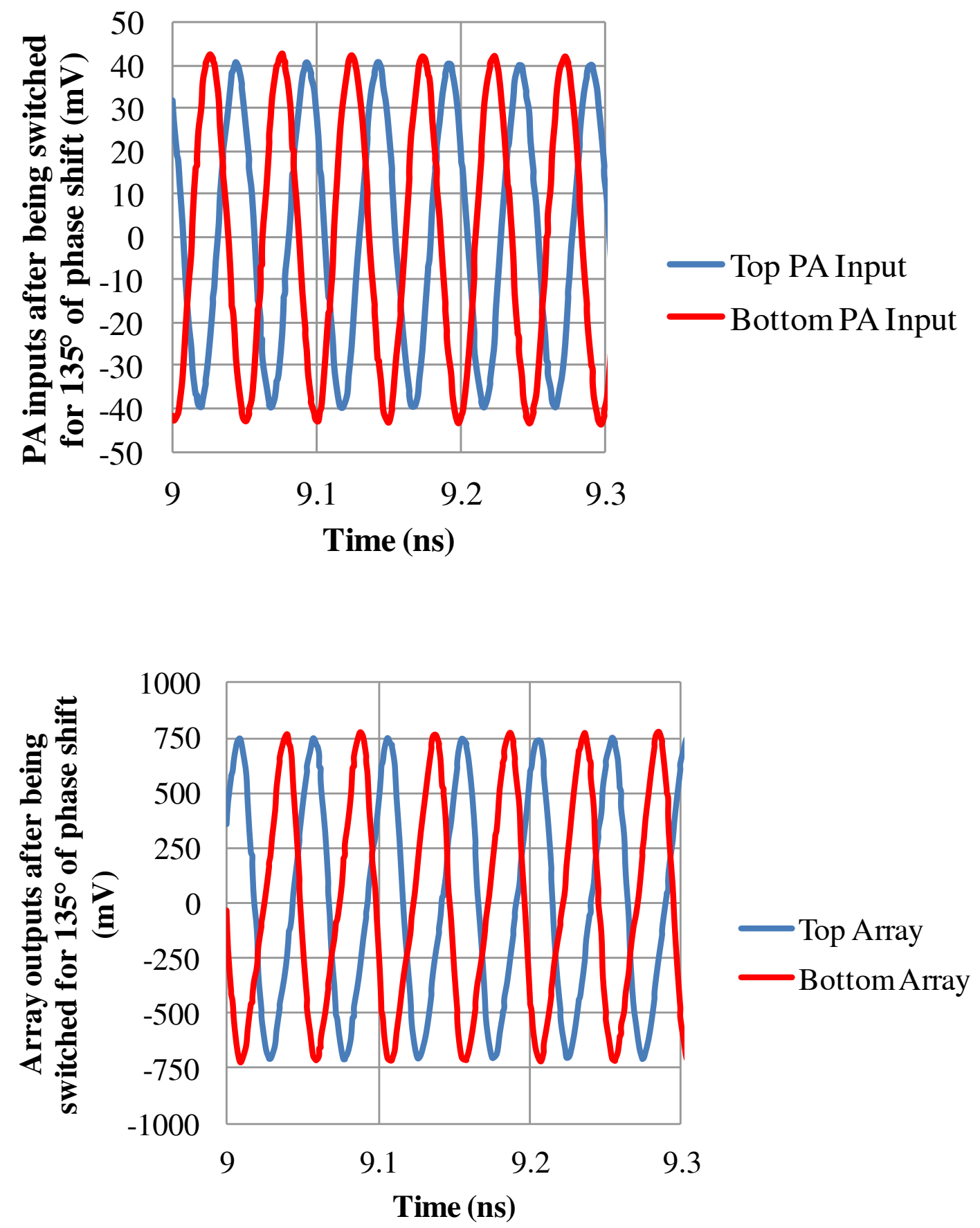

Figure 6.5 EM simulation results of the full system for the RTWO's $135^{\circ}$ signals 
The overall simulation results show that the signals provided to the antennas are symmetrical and the phase shift inaccuracy is lower than $5^{\circ}$ with a minimum value of $2.25^{\circ}$.

\subsection{Printed Circuit Board with Antennas}

Figure 6.6 shows a photo of the printed circuit board containing the antennas used in the transmitter. The two-layer PCB is realized on a Rogers RO4003 substrate, as described in Chapter 5. The total size of the printed circuit board is $8 \mathrm{~cm} \times 9 \mathrm{~cm}$.

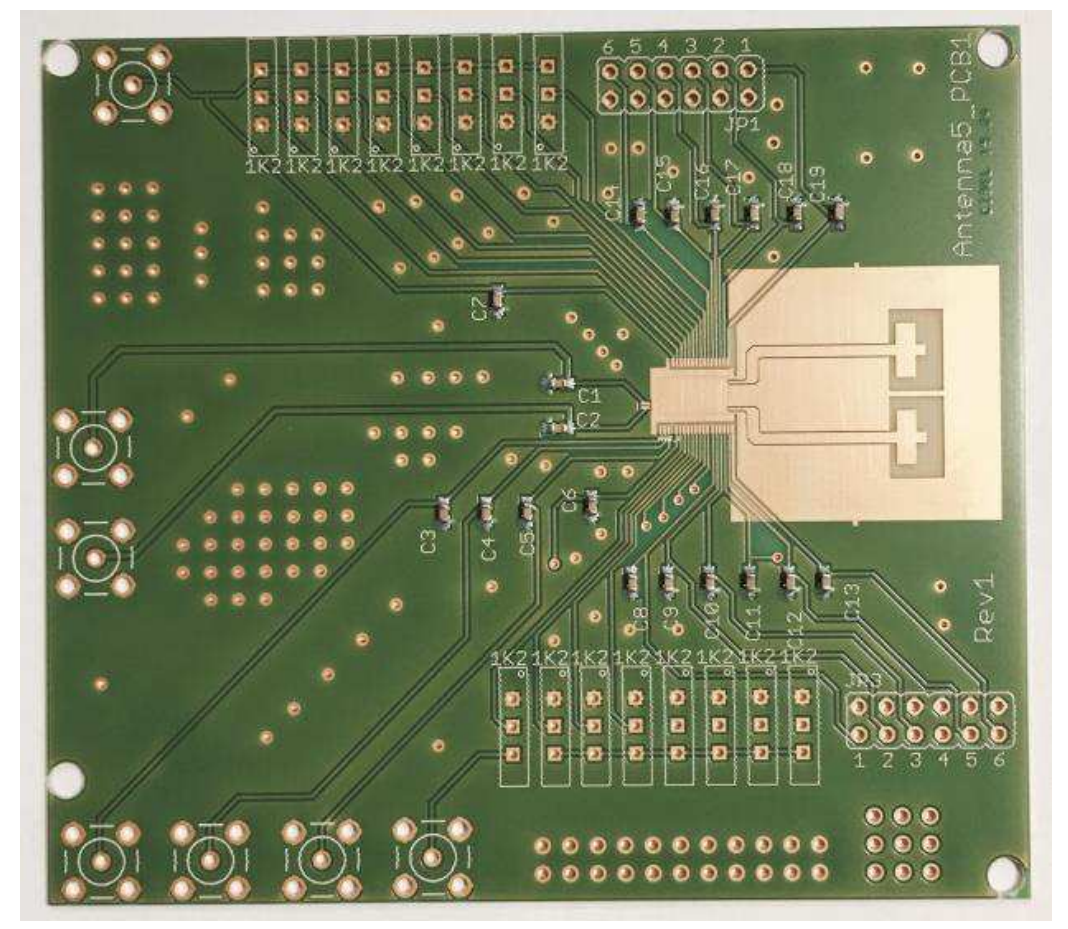

Figure 6.6 Photograph of the printed circuit board

The BiCMOS integrated circuit described earlier is intended to be connected to the printed circuit board using bond-wires. Ground metal is exposed on the printed circuit 
board where the integrated circuit is to be mounted, providing a reference for the silicon die.

The antennas, on the other hand, are designed to be fed by a coplanar waveguide, and so there is no ground under them. Therefore the other metal layer, on the back side of the board, which is normally also a ground layer is not present under the antennas.

A total of 37 of 74 pads on the IC are ground pads. This large number of ground pads is required to ensure that there is no potential difference between the grounds of the two circuits. Since the bond-wires used to connect the die to the PCB have approximately $0.8 \mathrm{nH} / \mathrm{mm}$ of inductance, this number of parallel bond-wires greatly reduces the ground inductance. This is required to ensure adequate performance and stability, particularly for the amplifiers.

\subsection{Measurement Results of the Full System}

In order to fully characterize our two-element-array transmitter, we have designed and fabricated three different printed circuit boards. Two of these boards are holding antennas which are used to measure the functionality of the full transmitter, while the third board is terminated to $50 \Omega$ SMA jacks in order to measure the performance of the full system without the effect of the antennas.

Figure 6.7(a) shows the photo of the printed circuit board holding SMA jacks and Figure 6.7(b) shows our measurement set up using LM317 voltage regulators and potentiometers inserted on two breadboards to DC bias the top and bottom power 
amplifiers. Each PA contains six DC pads to bias base and collector nodes of all three stages individually and two 12 pin headers, seen in Figure 6.7, are soldered on the printed circuit board for this purpose.

The other soldered components on the printed circuit board, seen in Figure 6.7(a), are SMB jacks to DC bias other on-chip components including the RTWO, buffers, inverters and on-chip switches.

There are a total of 16 slide switches, seen on the top and bottom side of the board, used to connect the $V_{d d}$ to the gate of the desired on-chip switch in order to do beamforming.

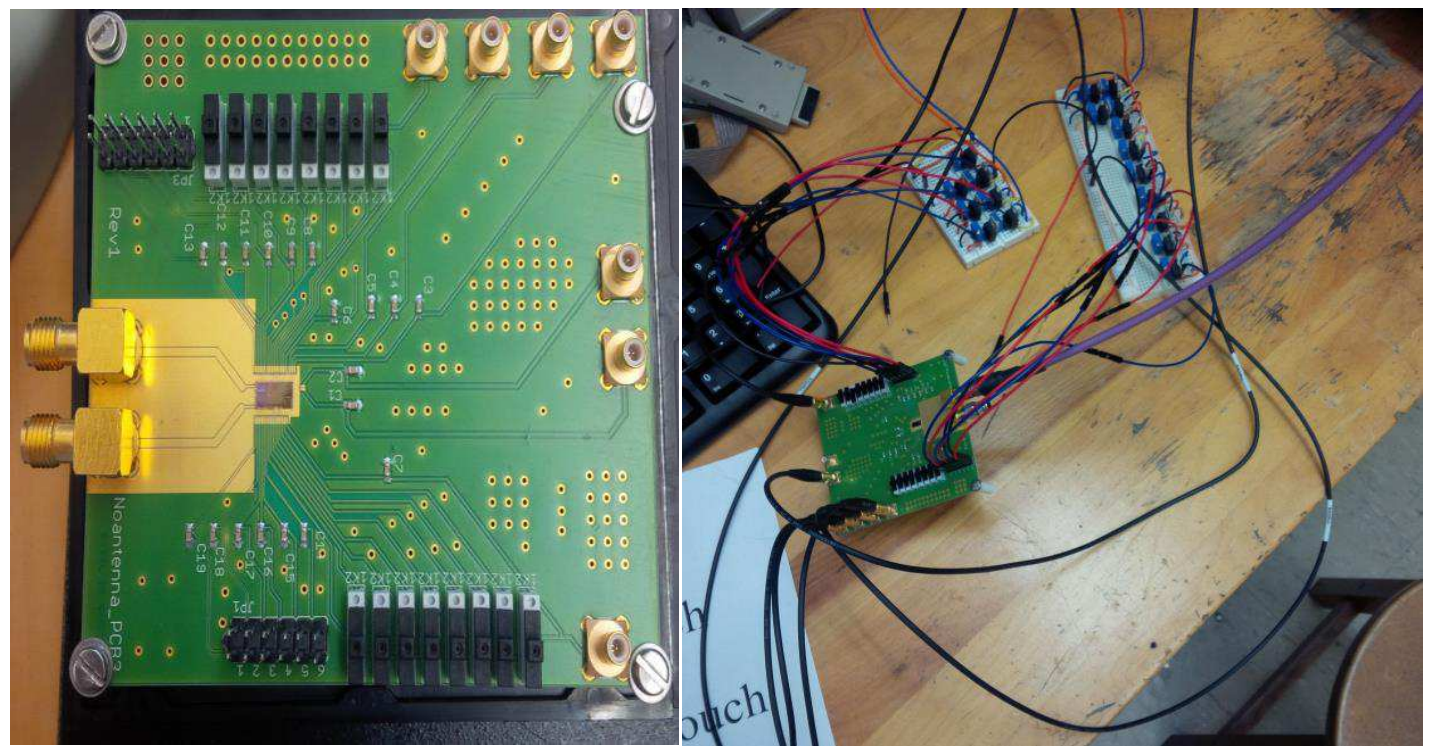

(a)

(b)

Figure 6.7 Photo of the printed circuit board and measurement setup. (a) printed circuit board (b) measurement setup 
Using this measurement setup, we have measured the output power of the PAs, the amplitude mismatch between the top and bottom arrays for different switching options, the tuning range of the full system and also the isolation between the PAs with the aid of HP8564E spectrum analyzer.

Figure 6.8 and Figure 6.9 show the output power of top and bottom elements, respectively. From Figure 6.8, the top element is providing $-1.67 \mathrm{dBm}$ of output power at 17.25 GHz. However, calibrating out the loss of the cable which is $1.33 \mathrm{~dB}$ in this case the value of output power goes up to $-0.34 \mathrm{dBm}$.

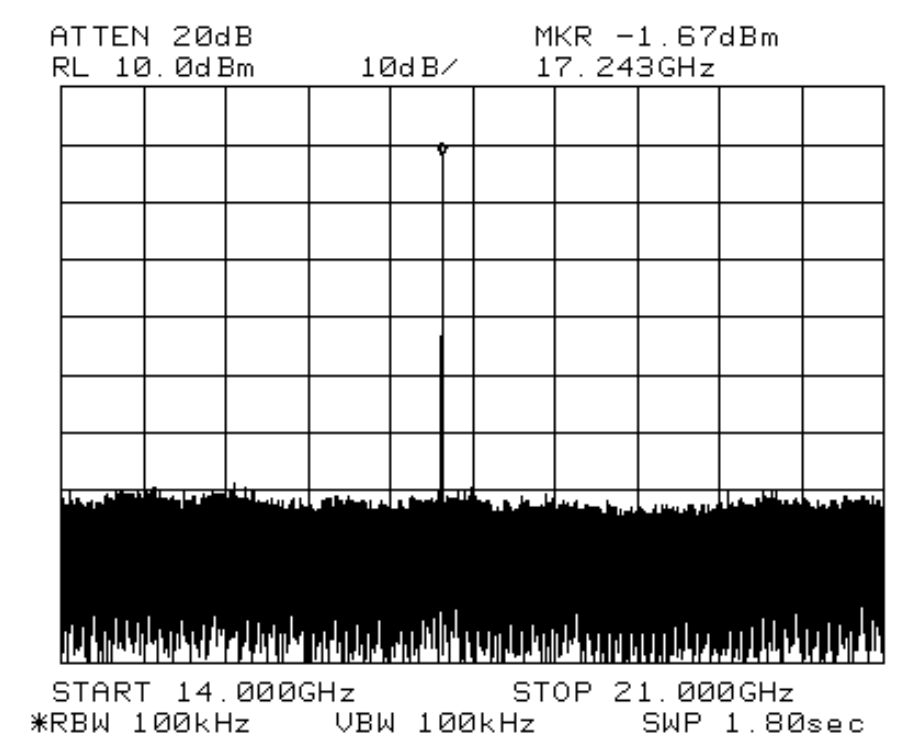

Figure 6.8 Output power of the top element while the bottom element is terminated to $50 \Omega$

From Figure 6.9, the output power of the bottom element is $-2.17 \mathrm{dBm}$ and after calibrating out the loss of the cable, this number goes up to $-0.84 \mathrm{dBm}$. 


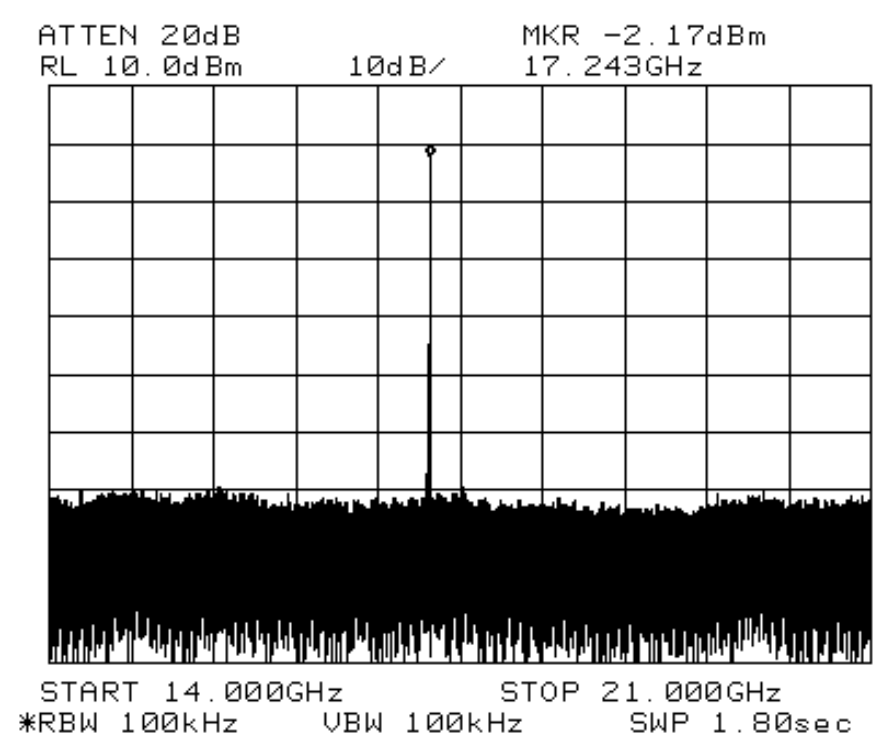

Figure 6.9 Output power of the bottom element while the top element is terminated to $50 \Omega$

The tuning range of the full system is also measured using the same printed circuit board. Figure 6.10, shows the output power of the top element, while the bottom element is terminated to $50 \Omega$, after changing the varactor's tuning voltage from $0 \mathrm{~V}$ to $2.5 \mathrm{~V}$. From this figure, the output frequency moves to $18.34 \mathrm{GHz}$ and the output power, after calibrating out the loss of the cable, is $-2 \mathrm{dBm}$.

From Figure 6.8 and Figure 6.10, we can state that the full transmitter shows $1.1 \mathrm{GHz}$ of tuning range while there is $1.66 \mathrm{~dB}$ of amplitude variation across this range. This can be due to the different loading of the PAs for different frequencies. However, the tuning range and the minimum and maximum frequencies matches the RTWO's measurement results and also our EM simulation results. 


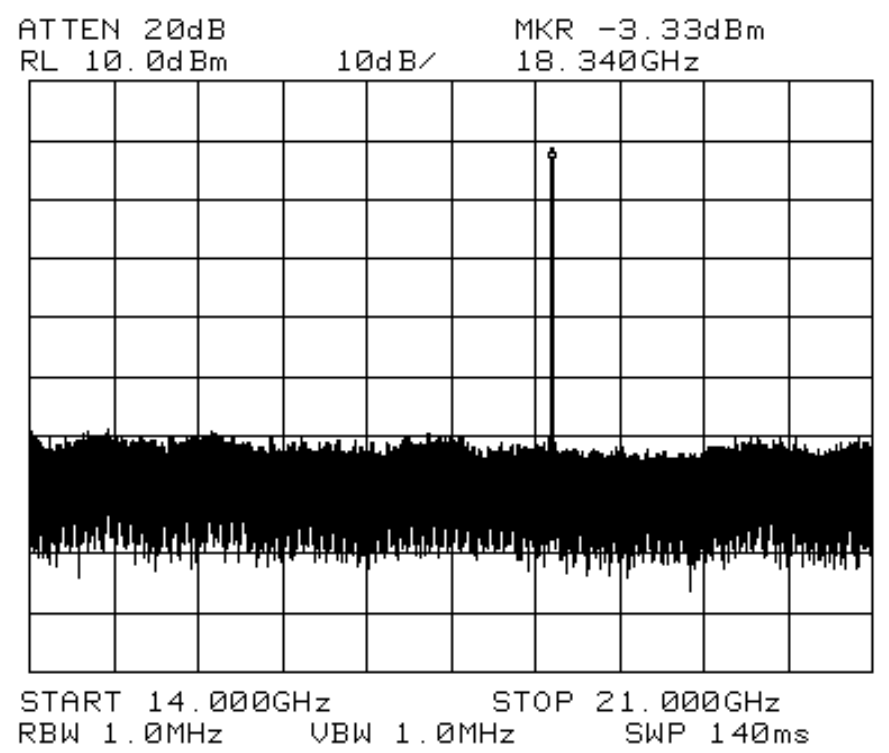

Figure 6.10 Output power of the top element for $V_{\text {tune }}=2.5 \mathrm{~V}$

The coupling between multiple PAs on the same die is quite important for integrated phased-array systems because it can affect the beam forming resolution. We have dedicated a physical distance of $1.4 \mathrm{~mm}$ between the two PAs in our layout and this amount of physical distance can improve the isolation.

In order to measure the isolation between the two elements, we have deactivated one element by turning OFF the switching bank of that element and activated the other element by turning on a switch from its switching bank. The isolation is determined by comparing the output power of the activated element with the output of the deactivated element.

Figure 6.11 shows the measured output power of the top element while all the switches are slid to the OFF position. From this figure and also comparing it with Figure 
6.9 , the isolation between the two elements is $39.33 \mathrm{~dB}$. The worst case measured isolation between the two elements, for other selected switches, was $20 \mathrm{~dB}$.

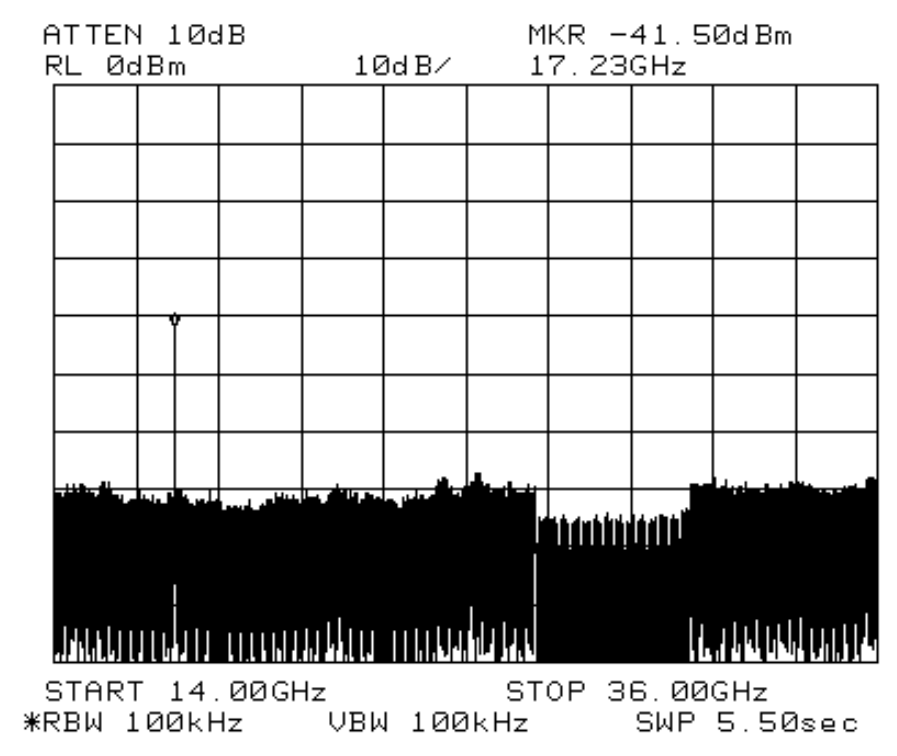

Figure 6.11 Measured output power of the top element while all switches are OFF

The small drift in the oscillation frequency seen in Figure 6.9 and Figure 6.11 is due to the fact that the RTWO is a free-running oscillator and it is not locked to any reference frequency. However, $1.1 \mathrm{GHz}$ of tuning range should take care of this problem.

\subsubsection{Phase Shift Measurement Using Power Combiner}

In order to measure the beam-forming capability of the full system, we have tried and tested two different approaches and the measurement results of both methods are presented in this chapter.

The first approach is the use of our own frequency domain phase shift measurement technique which has been explained in detail in Chapter 4. 
However, as has already been discussed in Chapter 4, using this approach the slope of the tangent line for small phase shifts between the two signals is quite small. For example, from Figure 4.11, the combined output power variation of the RTWO for phase shifts between $0^{\circ}$ to $45^{\circ}$, is only $0.7 \mathrm{~dB}$.

This means that in order to detect this amount of output power variation to determine small phase shifts using this approach, a very careful test setup calibration is required. However, knowing the fact that the precision of our available spectrum analyzer is $0.34 \mathrm{dBm}$, we have to calibrate the test setup for any loss equal or lower than $0.36 \mathrm{dBm}$ in order to be able to determine any phase shifts smaller than $45^{\circ}$.

All these make it almost impossible for us to measure the free-running RTWO's phase steps of $0^{\circ}$ and $45^{\circ}$ using our approach with the available lab equipment. As a result, the measurement results for the RTWO's phase shift of $90^{\circ}, 135^{\circ}$ and $180^{\circ}$ are presented below.

In order to measure $90^{\circ}$ of phase shift between the two elements, using the same printed circuit board holding SMA connectors, we have turned ON particular switches which are delivering the RTWO's outputs from $A_{1}$ and $B_{3}$ nodes, as shown in Figure 4.2, to the input nodes of the PAs. From Figure 4.2, these two signals should have the phase shift of $90^{\circ}$ or $270^{\circ}$ depending on the RTWO's direction of rotation. The unbiased signal rotation of an RTWO can occur in either clockwise or counter clockwise direction.

The first step is to measure the output power of each element, for the selected switches, individually while terminating the other element to $50 \Omega$. The output power of 
each element for these selected switches, namely $A_{1}$ and $B_{3}$, are shown in Figure 6.8 and Figure 6.9.

Figure 6.12 shows the combined output power of the transmitter using an Agilent $11667 \mathrm{~A}$ power combiner to combine the outputs of the top and bottom elements after being set for $90^{\circ}$ of phase shift. The details of the calibration procedure have been provided in Chapter 4.

From this figure the combined power is $-11.83 \mathrm{dBm}$ and we have used a cable with $2.88 \mathrm{~dB}$ loss to connect the output of the power combiner to the spectrum analyzer. This loss has to be taken into account in our final phase shift calculations.

In order to convert the combined amplitude information to the phase shift between the two signals, either a hand calculation, or the measurement setup simulation is required. By simulating the measurement setup, we can directly use the measured power values instead of converting them back to the voltage which is necessary for hand calculations.

Figure 6.14 shows simulation results using the test setup shown in Figure 6.13. For this setup, we have employed two phase-matched cables with $1.33 \mathrm{~dB}$ and $1.83 \mathrm{~dB}$ of loss to connect the outputs of the top and bottom elements to the power combiner. Another cable with loss of $2.88 \mathrm{~dB}$ has been used to connect the output of the power combiner to the spectrum analyzer 


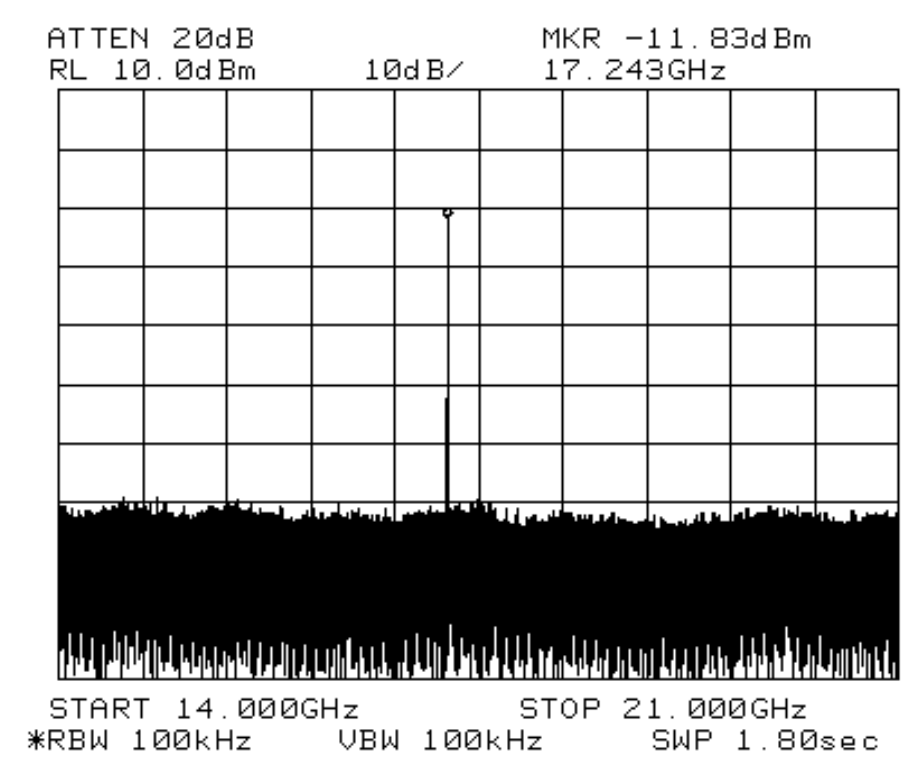

Figure 6.12 Measured combined output power of the transmitter after being switched for $90^{\circ}$ of phase shift

Our simulation results, shown in Figure 6.14 , predicts $106^{\circ}$ of phase shift for the target phase shift of $90^{\circ}$. This difference can be due to a few reasons. First of all we are expecting $\pm 5.5^{\circ}$ of phase error from our approach, using our available lab equipment, which has already been explained in Chapter 4 .

The other main reason for this phase difference can be the effect of wire-bonds which are used to connect the silicon die to the printed circuit board. These wire-bonds have different physical shapes and lengths and an accurate model for them is required in order to measure the phase shift using any approach. The last reason could be the mismatch loss between the outputs of the PAs, after being wire-bonded and routed to the right-side of the printed circuit board, and the spectrum analyzer. For these measurements, we have assumed that the outputs of the PAs, all the way to the SMA connectors, are matched to 
$50 \Omega$ and we did not include the effect of any mismatch loss in our simulation set up while in reality this matching is not perfect and can be different than $50 \Omega$.

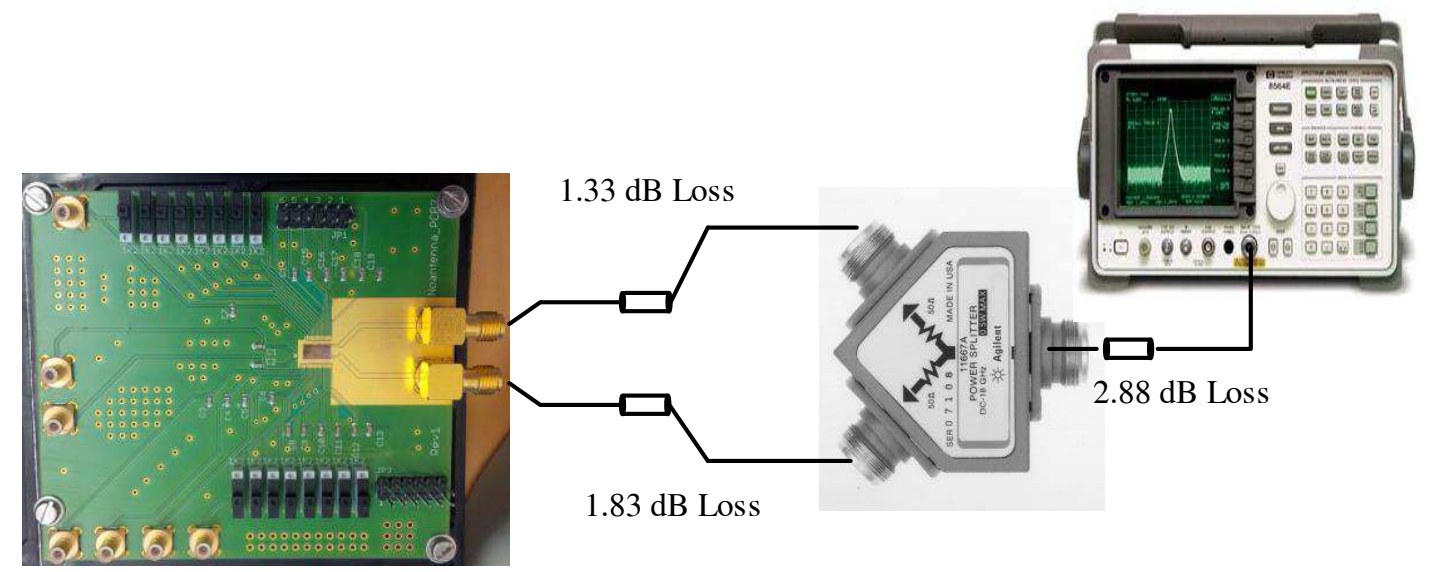

Figure 6.13 Measurement setup used to measure the phase shift using a power combiner

However, the phase error due to the mismatch loss between the PAs' outputs and the spectrum analyzer can be calibrated out since we are hoping to measure different phase shifts from our full system, unlike the first case explained in Chapter 4 for which we had access to $90^{\circ}$ of phase shift only. The value of this mismatch loss is expected to be the same for all phases and after measuring different phases using the same setup we should be able to approximate this value. 


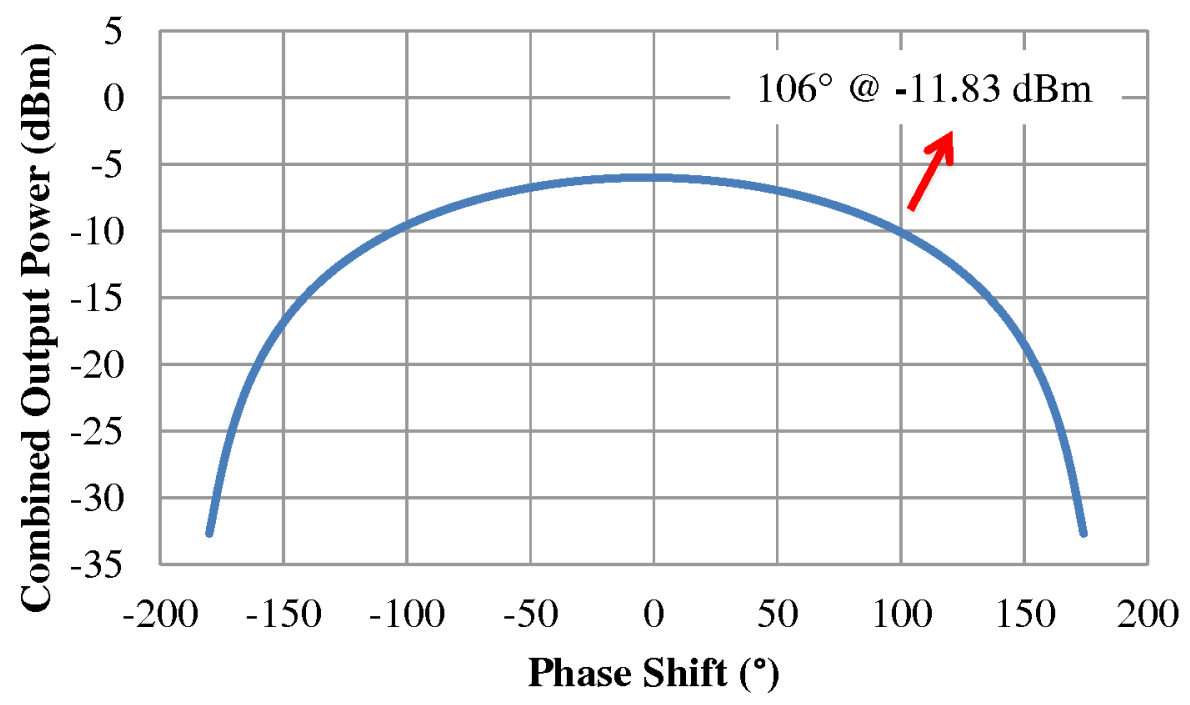

Figure 6.14 Simulated combined output power used to calculate $90^{\circ}$ of phase shift

We have repeated the same procedure for $135^{\circ}$ of phase shift. For this phase shift, the switches which are delivering the RTWO's output signals of $A_{I}$ and $A_{2}$ to the input of the PAs are turned ON. From Figure 4.2, these two outputs should provide $135^{\circ}$ or $225^{\circ}$ of phase shift depending on the RTWO's direction of rotation.

Figure 6.15 shows the output of the bottom element, we kept the same $A_{l}$ switch, shown in Figure 6.8, ON for the top element, after being switched for $135^{\circ}$ of phase shift.

From this figure the output power of the bottom element is $-4.33 \mathrm{dBm}$ when $A_{2}$ switch is selected. From Figure 6.8 the output of the top element for $A_{I}$ switch is $-1.67 \mathrm{dBm}$.

Figure 6.16 shows the combined output power of two elements after being switched for $135^{\circ}$ of phase shift. From this figure the combined output value is $-15.17 \mathrm{dBm}$. 


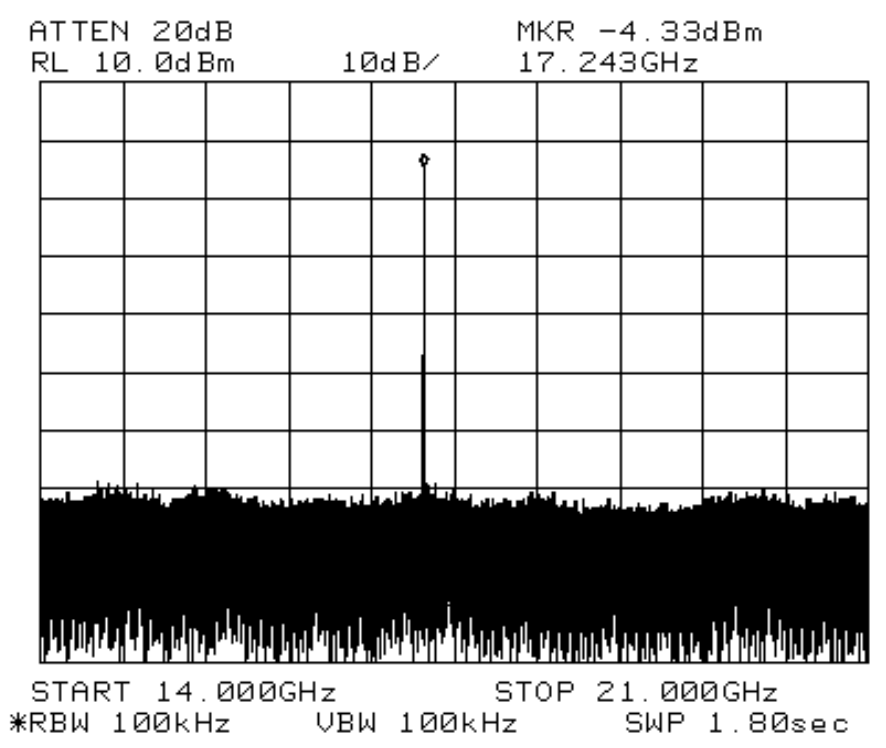

Figure 6.15 Output of the bottom element while the $A_{2}$ switch is $\mathrm{ON}$

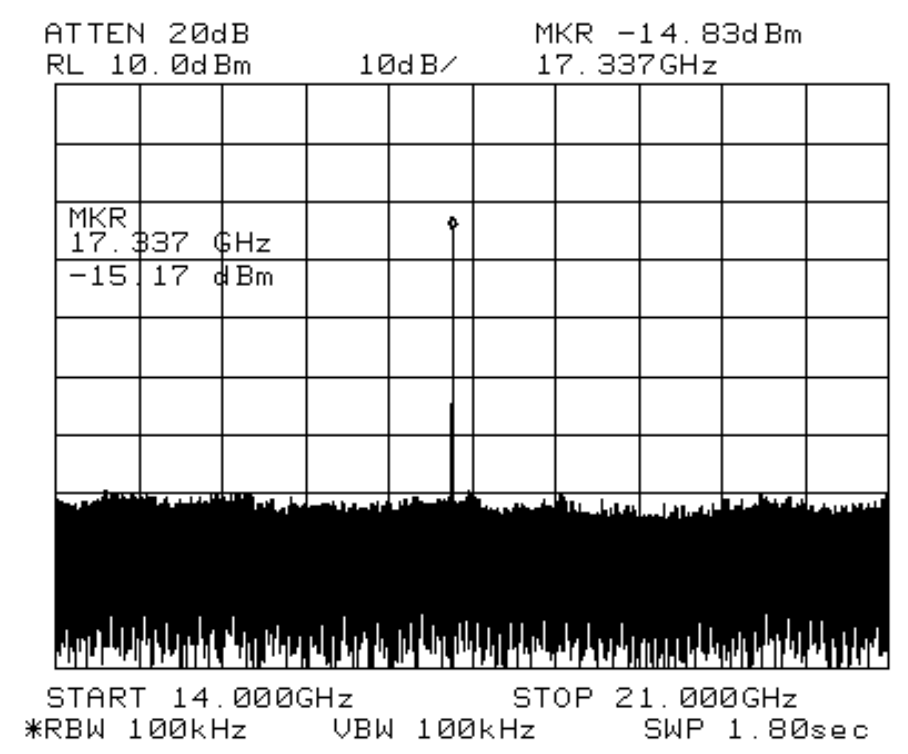

Figure 6.16 Combined output power of the top and bottom elements after being switched for $135^{\circ}$ of phase shift

Following the same procedure and simulating the measurement setup, this value of combined output power corresponds to $144^{\circ}$ of phase shift. 
The same steps have been followed for $180^{\circ}$ of phase shift between $A_{1}$ and $B_{1}$ nodes of the RTWO. The measured output powers of these nodes are $-1.67 \mathrm{dBm}$ and $-3 \mathrm{dBm}$ while the combined output power is $-20 \mathrm{dBm}$. This corresponds to $173^{\circ}$ of phase shift.

Figure 6.17 shows the summary of our measurement results using our approach. The red dots shows the theoretical results for $0^{\circ}, 45^{\circ}, 90^{\circ}, 135^{\circ}$ and $180^{\circ}$ of phase shift. The purple dots are our EM simulated results and the green dots are our measured results for $45^{\circ}, 90^{\circ}, 135^{\circ}$ and $180^{\circ}$ of phase shift using the power combiner.

As can be seen from this figure, there is a $13.5^{\circ}$ of phase error between our measured and EM-simulated results for RTWO's phase shift of $45^{\circ}$. This error is big and perhaps it is not negligible but we were expecting relatively big phase error for phase shifts smaller than $45^{\circ}$ using this approach. The smallest phase error is seen between the measured and simulated results for $135^{\circ}$ of phase shift and this error is $4^{\circ}$.

However, the above obtained results are showing the functionality of the full system meaning that the transmitter is working properly and the signal can get out of the PAs with the predicted frequency, phase and output power. Our beam-forming measurement also shows that we can select the desired output of the RTWO to be delivered to the PAs and there is good isolation between the two PAs. 


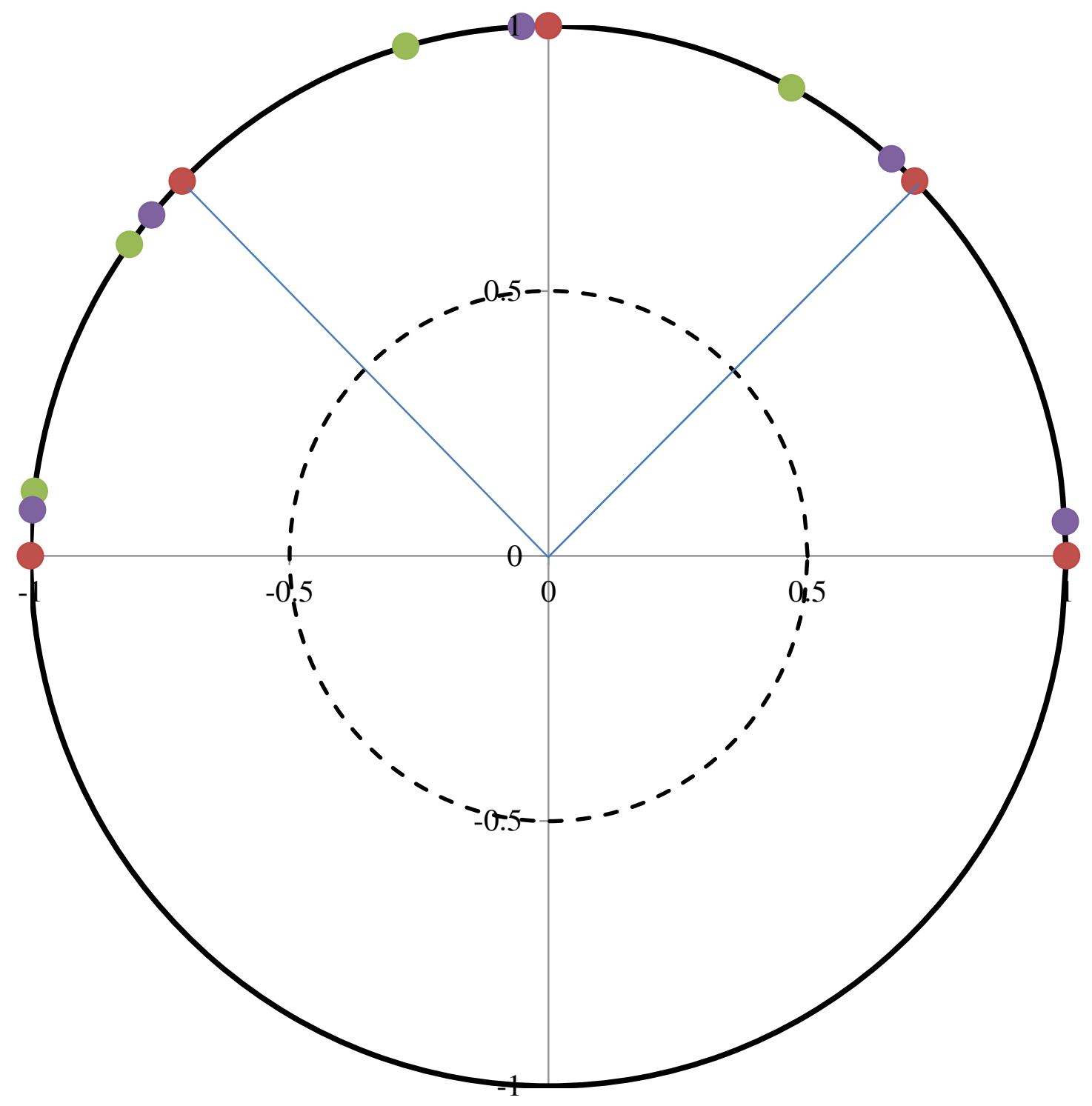

Figure 6.17 Comparison of theoretical (red), EM-simulated (purple) and measurement results (green) of phase shift using a power combiner for phase shift measurement 


\subsubsection{Beam-Forming Measurement Using Physical Antennas}

The last measurement needed to show the functionality of the full transmitter including the antennas is to measure the beam-forming ability of the system with the aid of the antennas. This is the only accurate way to measure the beam-forming capability of the system.

According to our measurement results using SMA jacks, the transmitter frequency for tuning voltage of $0 \mathrm{~V}$ is $17.24 \mathrm{GHz}$. Table 7 summarizes the radiation angle of the transmitter for different RTWO phase shifts calculated using (5.7) and transmitted frequency of $17.24 \mathrm{GHz}$.

Table 7 Summary of the calculated radiated angles from the RTWO's phase shift

\begin{tabular}{|c|c|}
\hline RTWO Phase Shift & Radiated Angle \\
\hline$\Delta \varphi$ & $\boldsymbol{\theta}$ \\
\hline $0^{\circ}$ & $0^{\circ}$ \\
\hline $45^{\circ}$ & $15.78^{\circ}$ \\
\hline $90^{\circ}$ & $32.95^{\circ}$ \\
\hline $135^{\circ}$ & $54.65^{\circ}$ \\
\hline $180^{\circ}$ & $\sin ^{-1}(1.08)$ \\
\hline
\end{tabular}

Figure 6.18 and Figure 6.19 show the HFSS geometry for two antennas beside each other and its simulated results showing the radiation pattern of two antennas for different phase shifts between them. 
In Figure 6.19 the red pattern refers to $0^{\circ}$ of phase shift, the green pattern refers to $45^{\circ}$ of phase shift, the blue pattern refers to $90^{\circ}$ of phase shift and finally the orange pattern refers to $135^{\circ}$ of phase shift between the inputs of the antennas.

As can be seen from this figure, as the input phase shift between the two antennas, $\Delta \varphi$, gets bigger the second lobe starts to show up. For $0^{\circ}$ of phase shift between the two antennas, the radiation pattern contains only the main lobe pointing to $0^{\circ}$. However, for $135^{\circ}$ of input phase shift between the antennas the side lobe gets almost as strong as the main lobe while it is still pointing at the desired radiation angle of $54^{\circ}$, according to Table 7.

In our case and for the particular RF frequency of $17.24 \mathrm{GHz}$ and antenna spacing of $8 \mathrm{~mm}$, the maximum meaningful input phase shift between the antennas is $165.5^{\circ}$ and basically after this phase shift, the main beam reappears on the wrong side and the signals are not adding coherently anymore for the desired direction.

It should be noted that for the PCB design we have tapered the piece of line used to connect the antenna to the die, unlike what is seen in Figure 6.18, in order to avoid any kind of discontinuity and ensure a smooth connection to the rest of the system. 


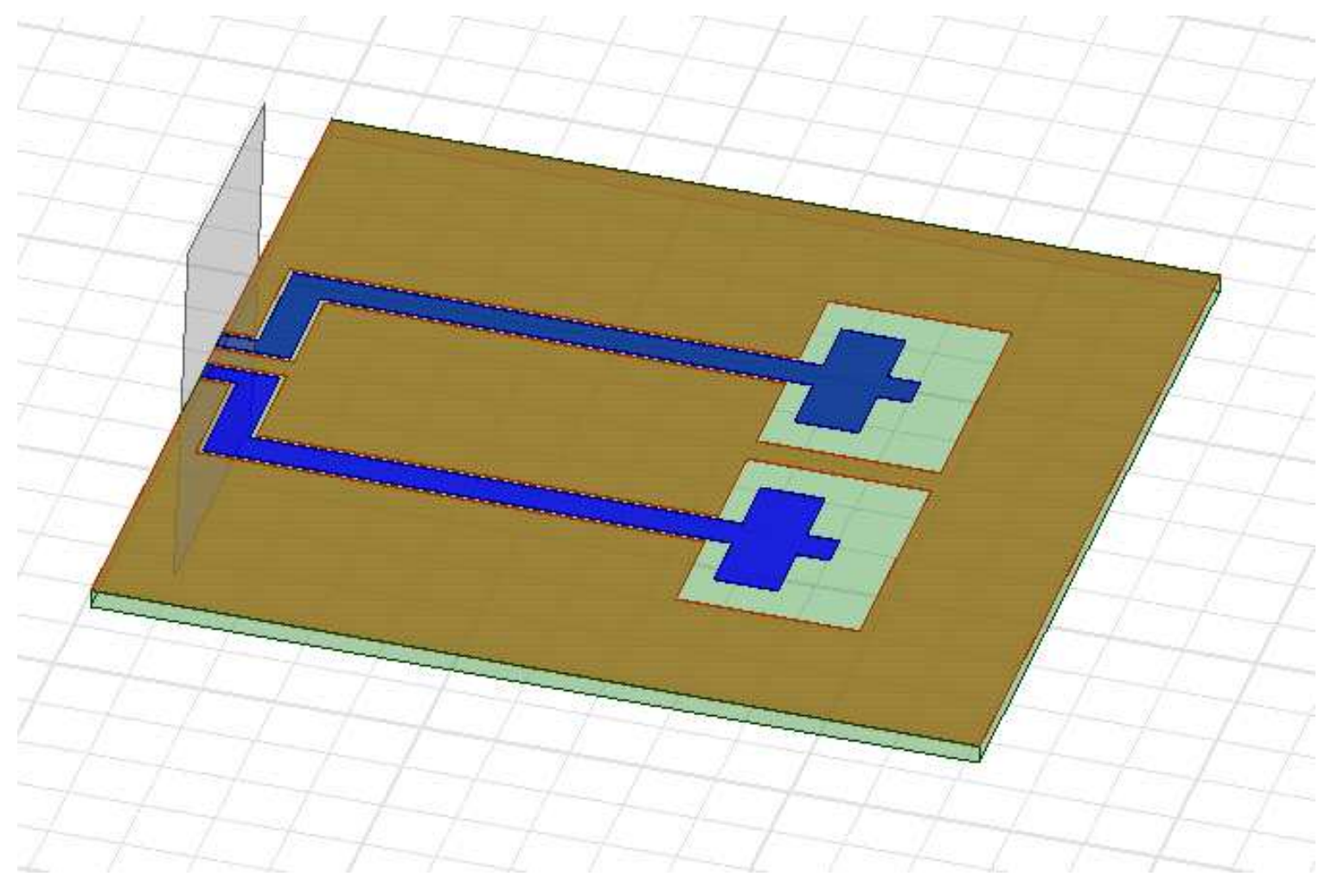

Figure 6.18 HFSS geometry for two antennas being $8 \mathrm{~mm}$ apart

Since there are only two antennas in our antenna array, both in simulation and the final product, the main lobe is quite wide and the beam steering resolution is not that high and it is only sufficient to show the proof of the concept. 


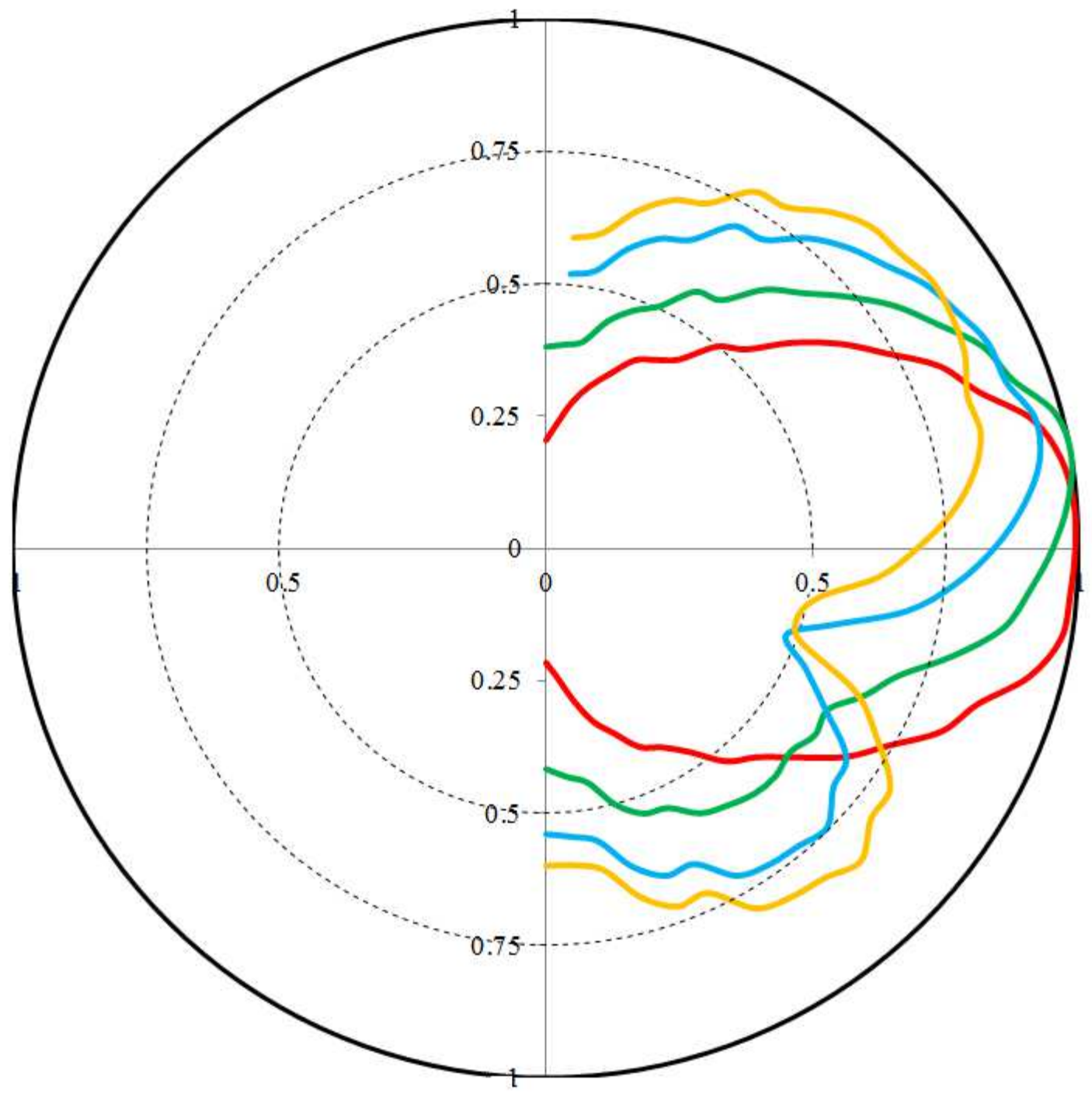

Figure 6.19 HFSS simulation results for the radiation pattern of two antennas beside each other fed by signals with different phase shifts 


\subsubsection{Array Output Power and Amplitude Mismatch}

\section{Measurement}

In order to measure the radiated power of the full transmitter and also the amplitude mismatch between the top and bottom elements after being switched for different phase shifts, we have used a BAE Systems' H-1498 horn antenna which has a frequency range of $2 \mathrm{GHz}$ to $18 \mathrm{GHz}$ and gain of $11 \mathrm{dBi}$ for its high band range.

Since the RTWO's oscillation frequency is far away from other known frequencies in free space like mobile, WiFi or radio, we have done this measurement outside of an anechoic chamber and in the lab while holding the horn antenna one meter away from our antennas, basically from our PCB, in order to be able to measure the far field radiation pattern or the transmitter.

Figure 6.20 shows our measurement setup. As can be seen from this figure, the horn antenna is attached to a long stick to be hold on top of our PCB which has to be connected to DC sources in order to bias the circuit. In order to measure the radiated pattern of each power amplifier and also the EIRP, we kept the distance between the transmitter and receiver antennas constant and we turned on the off-chip switches one by one to deliver the RTWO's output phases to the antennas, while keeping the angle between the two antennas zero.

As shown in Figure 5.26, our designed patch antennas are radiating in Z-direction and the PCB kept in the constant position on XY-plane for this measurement. 


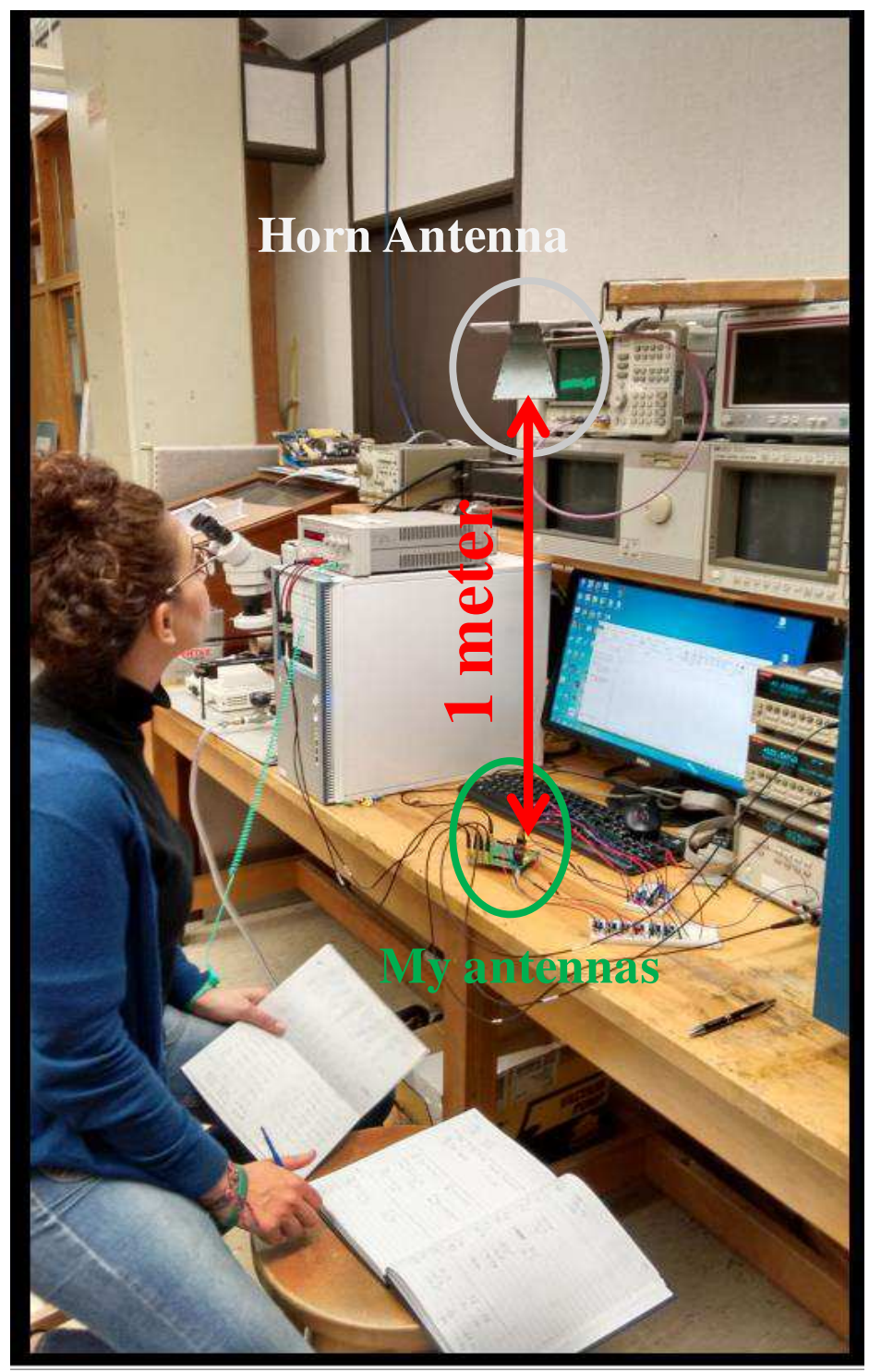

Figure 6.20 Measurement setup used to measure the output power and amplitude mismatch

Using (6.1) shown in [63], and measuring $-33.5 \mathrm{dBm}$ of power (after subtracting the loss of the cable) from the receiver antenna, the transmitted power, including the gain of both transmitter and receiver antennas, is $23.67 \mathrm{dBm}$.

$$
P_{r}=P_{t}-20 \log \frac{4 \pi f d}{c}
$$


where $P_{r}$ is the received signal power, $P_{t}$ is the transmitted signal power including the gain, $f$ is the frequency, $c$ is the speed of the light and $d$ is the distance between the two antennas.

In order to calculate the transmitted power of both elements and also the power amplifiers, we need to know the gain of the transmitter antenna.

Figure 6.21 shows the radiation pattern of each antenna, simulated in HFSS, after being extended and tapered on the PCB. As seen in this figure, the gain of the antenna is $3.43 \mathrm{~dB}$ and it is radiating in both directions since there is no reflector under the antenna.

Knowing the gain of the transmitter antenna and the receiver antenna, $11 \mathrm{dBi}$ in our case, the output power of the transmitter excluding the gain of the antennas can be calculated based on (6.2), [64].

$$
\begin{aligned}
P_{\text {Transmitter }}= & P_{t}-G_{t}-G_{r}=23.67 \mathrm{dBm}-3.43 \mathrm{~dB}-11 \mathrm{~dB} \\
& =9.24 \mathrm{dBm}
\end{aligned}
$$

where $G_{t}$ and $G_{r}$ are the gains of the transmitter and receiver antennas, respectively.

The EIRP of two antennas being apart by $\lambda / 2$ goes up by $20 \log 2$ which is $6 \mathrm{~dB}$. However in our case the antennas are apart from each other by more than $\lambda / 2$ and this combining effect should be less than $6 \mathrm{~dB}$. As a result in our case the full transmitter output power, from both elements, is $+9.24 \mathrm{dBm}$ and the output power of each power amplifier is close to $+3.5 \mathrm{dBm}$ which is higher than the $1-\mathrm{dB}$ compression point of the PAs. 

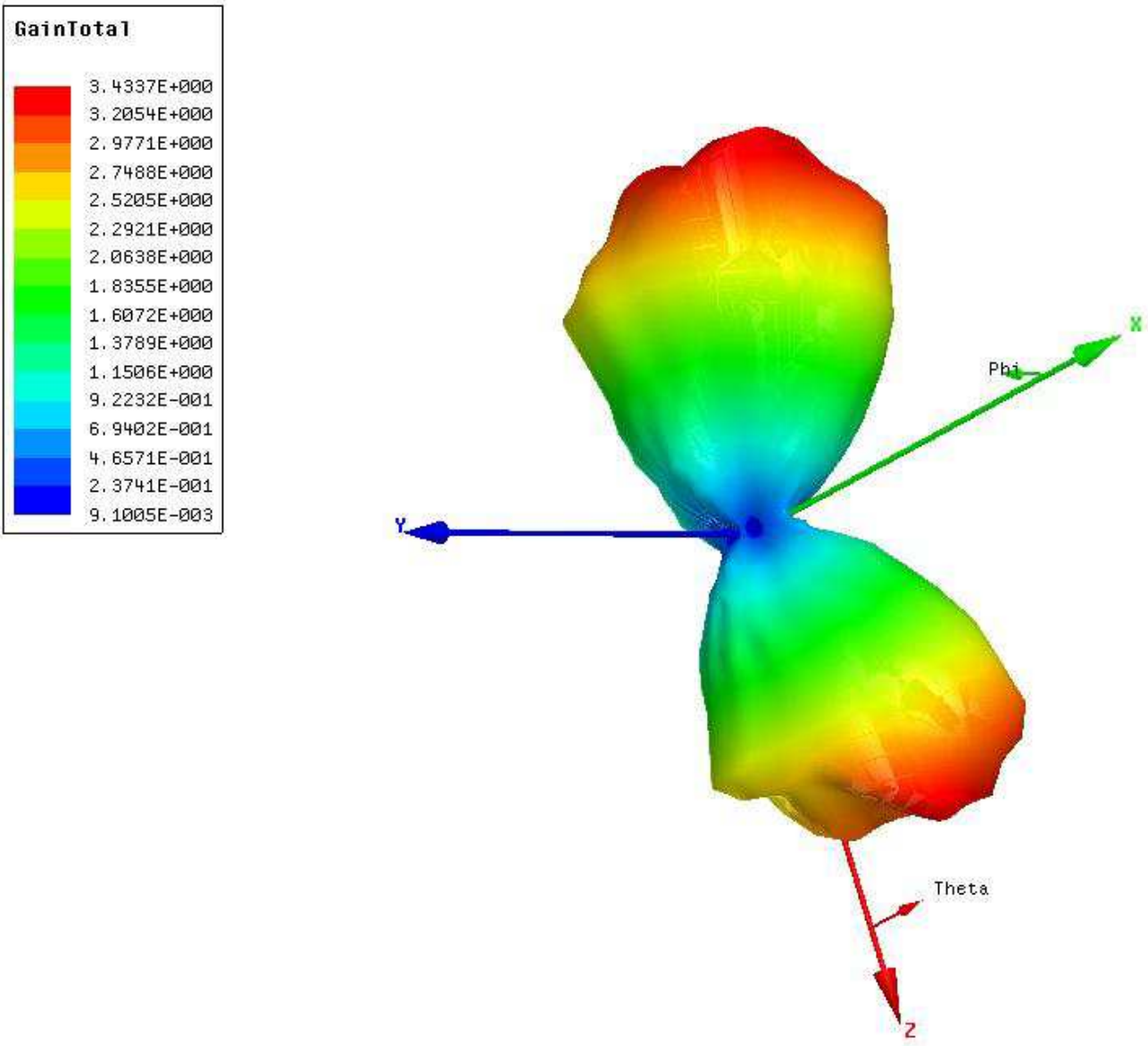

Figure 6.21 Radiation pattern of the antenna after being extended and tapered on the PCB

After measuring the output power of all 16 possible switching while keeping the distance and angle between the transmitter and receiver antennas constant, we noticed the worst-case amplitude mismatch of $2 \mathrm{~dB}$ between the two elements switched for different phases.

However, this value of amplitude mismatch can be reduced down to $0.75 \mathrm{~dB}$ by using different switch configuration for the same phase step thanks to our switching bank topology. 


\subsubsection{Beam-Forming Measurement}

In order to measure the beam-forming capability of the full system, we have to keep the physical distance between the center of the transmitter and receiver antennas constant while electronically switching to the desired phase and mechanically rotating the transmitter antenna to get the maximum amplitude at the desired angle and show the beam forming capability.

Figure 6.22 - Figure 6.25 shows measurement results for RTWO phase shifts of $0^{\circ}, \pm$ $45^{\circ}, \pm 90^{\circ}$ and $135^{\circ}$. According to Table 7 these phase shifts correspond to $0^{\circ}, \pm 15.78^{\circ}$, $\pm 32.95^{\circ}$ and $54.65^{\circ}$, respectively.

Comparing our measurement results with Figure 6.19, we can find the same kind of trend for different phase shifts. For example, our measured radiation pattern for $0^{\circ}$ of phase shift is a main lobe pointing to $0^{\circ}$. However as we increase this phase shift, the side lobe starts to appear and is almost as strong as the main lobe for phase shifts bigger than $135^{\circ}$. This effect has been noticed and reported both in HFSS simulation and in our measurements.

The RTWO is providing eight different phases with a $45^{\circ}$ step size and the beamsteering resolution of our two-element array transmitter is $15.78^{\circ}$ since the spacing between our antennas is bigger than $\lambda / 2$. The antenna spacing is bigger than $\lambda / 2$ because we have used coplanar structure for the design of our patch antennas and we needed some extra spacing between the radiating elements of the antennas to put the ground plane. 


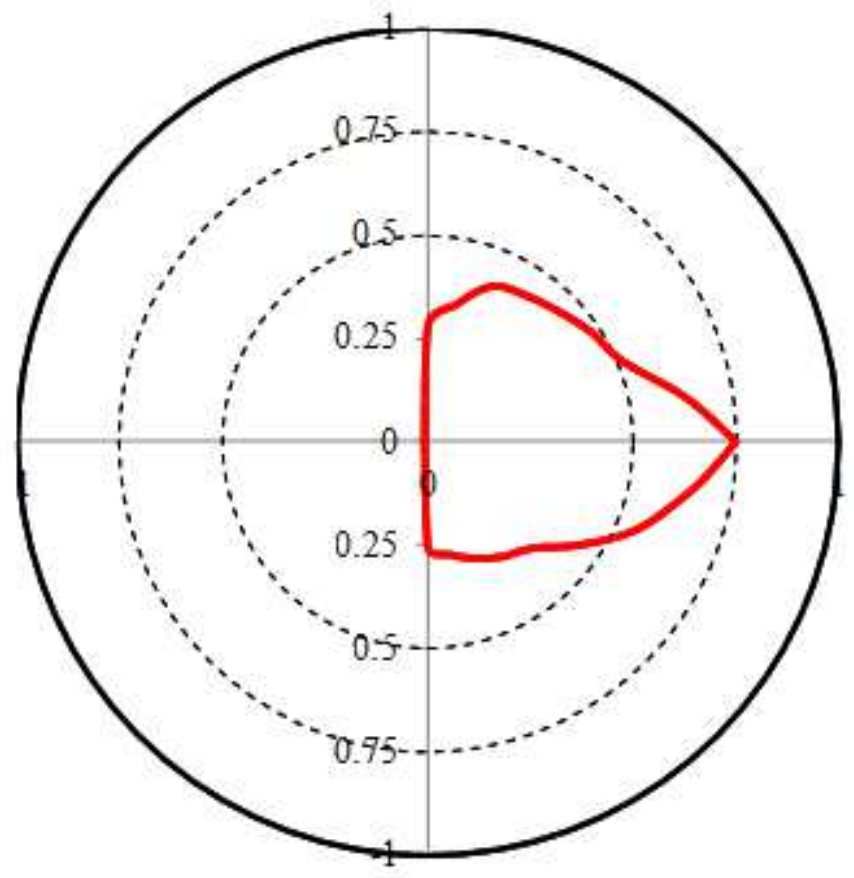

Figure 6.22 Measured radiation pattern for radiated angle of $0^{\circ}$

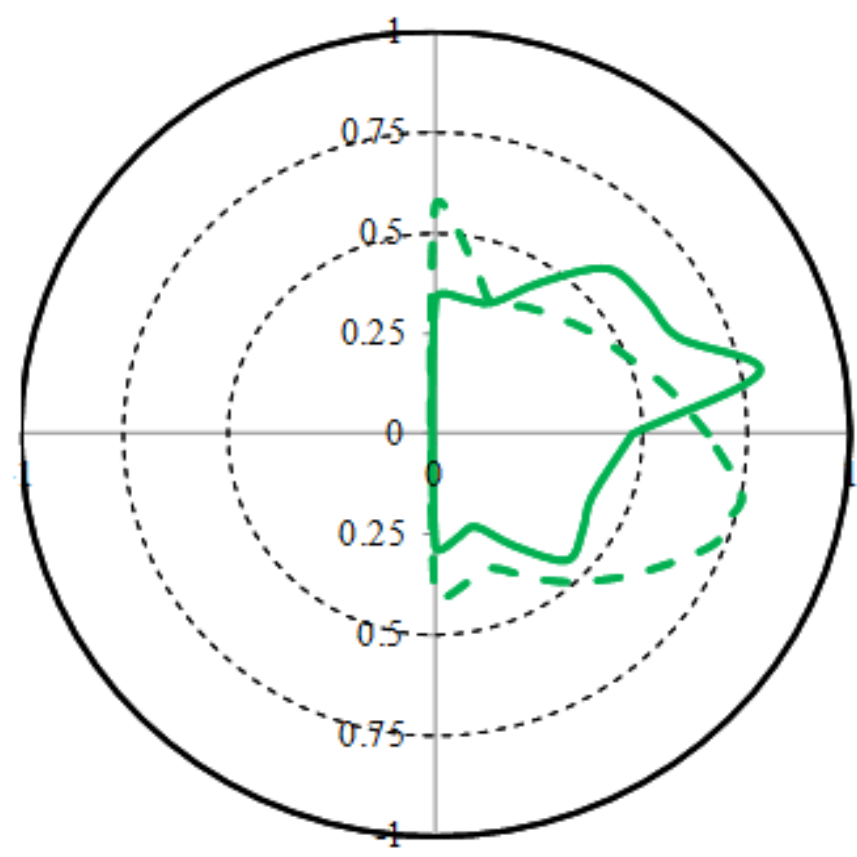

Figure 6.23 Measured radiation pattern for radiated angle of $\pm 15.78^{\circ}$ 


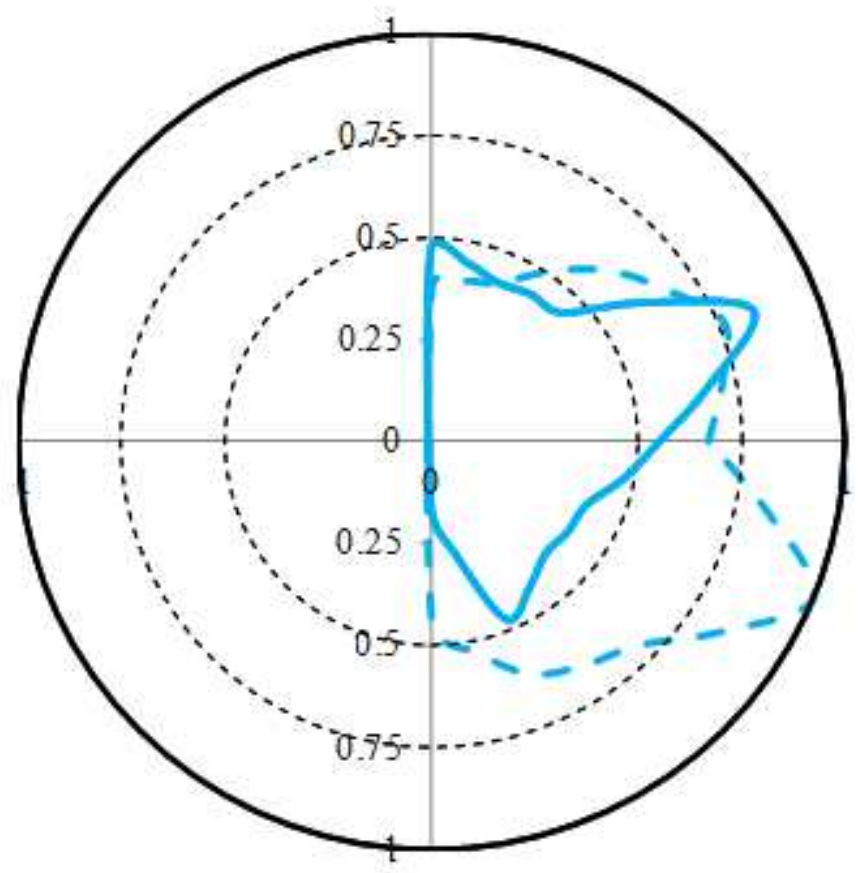

Figure 6.24 Measured radiation pattern for radiated angle of $\pm 32.95^{\circ}$

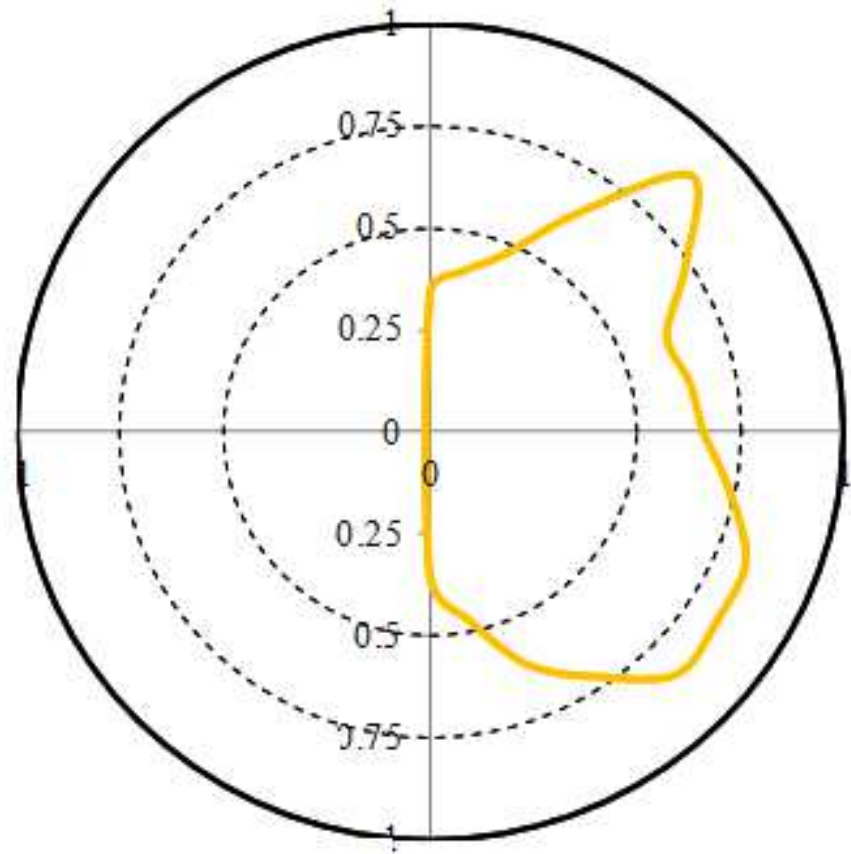

Figure 6.25 Measured radiation pattern for radiated angle of $54.65^{\circ}$ 
Our measurement results show only $5^{\circ}$ of phase error which can be due to silicon die phase error coming from the RTWO or different arms of the distribution network and also our measurement setup and signal being reflected from the bench. We did the measurement manually, with the aid of a PCB holder and a protractor, and our finest rotation angle was $11^{\circ}$. However, according to Table 7 , the radiation angle for $45^{\circ}$ of phase shift should be $15.76^{\circ}$ which is almost $5^{\circ}$ higher than our finest rotation angle.

Figure 6.26 shows measured radiation patterns of all six phases on the same plot.

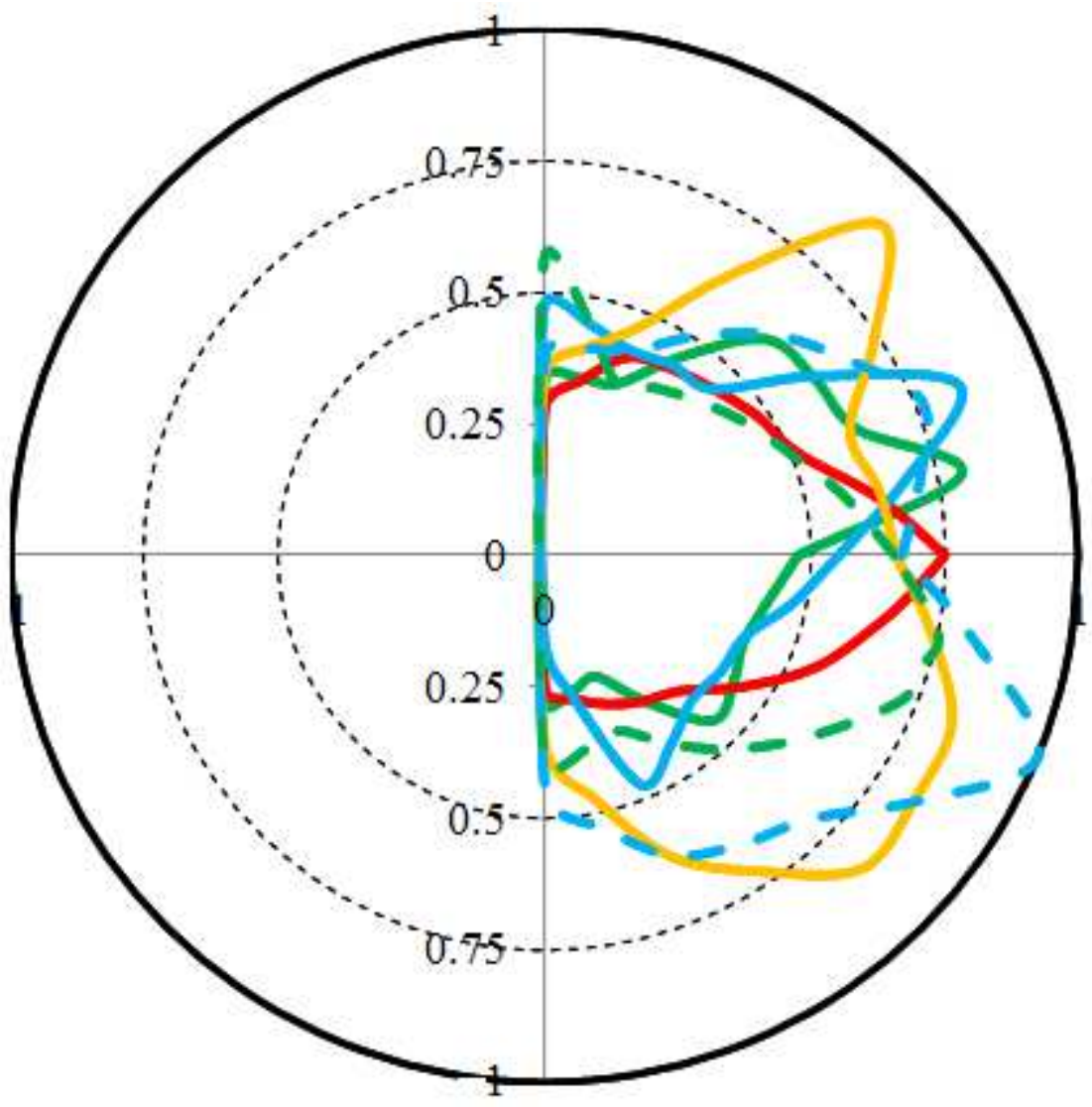

Figure 6.26 Radiation pattern of the antennas showing the beam-forming capability of the twoelement array transmitter 
This measurement along with our first measurement which was mainly focused on amplitude measurement show that the full monolithic Ku-band two-element-array transmitter connected to two off-chip antennas wire-bonded on the PCB, is fully functional and its performance is in close agreement with our EM-simulation results.

\subsection{Summary}

In this chapter, the full system functionality has been described. In order to demonstrate the system's beam-forming capability, a minimum of two antennas are needed. These antennas are designed on a printed circuit board, onto which the silicon die is bonded.

The total power consumption of the full two-element array, including all eight buffers of the RTWO, is $883.5 \mathrm{~mW}$. As mentioned earlier in the chapter the RTWO is functional with $2.5 \mathrm{~V}$ source and this reduces the total power consumption to $793 \mathrm{~mW}$. This power consumption is lower than other similar designs [9], [36] even after scaling down their power consumption to correspond to a two-element array.

The full functionality of the system is measured and the results are shown in this chapter. The maximum EIRP of our two-element transmitter is $+9.27 \mathrm{dBm}$ and it is capable of radiation at seven different desired angles including $0^{\circ}, \pm 15.78^{\circ}, \pm 32.95^{\circ}$ and $\pm 54.65^{\circ}$. Since the spacing between the two antennas is bigger than $\lambda / 2$ in our case, the beam-steering resolution is worse than the ideal case for LO-path phase shift step size of $45^{\circ}$. 
The EIRP of the transmitter is $+9.27 \mathrm{dBm}$ which is $4 \mathrm{~dB}$ lower than our EMsimulation results. This difference can be due the gain of the power amplifiers being $2 \mathrm{~dB}$ lower than simulations and also higher ground inductance of the full system which can reduce the output power. Even though the effect of $0.5 \mathrm{nH}$ inductance to model the parasitic effect of the wire-bonds is included in the EM-simulation setups, in the final product the wire-bonds are slightly longer than the minimum length.

The system shows the beam-forming capability after being tested with two different methods. The first method used to measure the beam-forming was our frequency domain phase measurement technique using a power combiner instead of antennas. Even though this technique shows some accuracy problems especially for small step sizes, but it showed that the phase of the RTWO gets out of the power amplifiers and the system can do the beam-forming.

The second approach to measure the beam-forming was the use of physical antennas to measure the radiation pattern of the two antennas, after rotating the angle of the transmitter antennas with respect to the receiver antenna. This final measurement showed the beam-forming capability for six different phases.

To the best of our knowledge this is the first phased array system utilizing an RTWO for LO-phase shifting and we have shown the proof of the concept with our measurement results. 


\section{Chapter 7 Conclusions}

This thesis has provided an overview of the three main categories of transmissionline-based oscillators. After some analysis of phase noise and oscillator characteristics, it was determined that rotary travelling-wave oscillators offer the potential to achieve high output power, low phase noise and can offer multiple phases which are needed in phasedarray systems.

The RTWO topology was analyzed to determine its operating characteristics, including oscillation frequency, output power and phase noise. The phase noise for a bipolar RTWO has not been previously covered in the scientific literature until now. Further to this analysis, an RTWO was designed in a BiCMOS technology. One advantage of this particular RTWO compared to others is its high output power. This reduces the need for amplification later in the transmitter, thereby reducing system power consumption.

As well, a new, innovative phase shift measurement technique has been proposed. This technique was required because of the lack of availability of a high-frequency oscilloscope, and the new technique is more accessible, particularly in university labs.

The use of an RTWO in a phased-array transmitter is novel to the best of our knowledge. To employ the RTWO in a phased-array transmitter, several other components are needed, including a distribution network, control unit, power amplifier and antennas. The design of each of these components and their integration has been 
described. Simulation results of the whole system have been presented and compared to other similar designs which shows the functionality of the full system and the fact that our simulation results are in close agreement with our measurement results.

Finally, the full functionality of the Ku-band two-element array transmitter was measured and the results were presented. The maximum EIRP of our two-element transmitter is $+9.27 \mathrm{dBm}$ and it is capable of radiating at seven different desired angles with beam-steering resolution of $15.78^{\circ}$. Since the spacing between the two antennas in our design is slightly larger than $\lambda / 2$, the beam-steering resolution is worse than the ideal case for an LO-path step size of $45^{\circ}$.

The measured EIRP is $4 \mathrm{~dB}$ lower than the EM-simulation results. This difference is due to the gain of the power amplifiers being $2 \mathrm{~dB}$ lower than expected and also higher ground inductance of the full system, due to the relatively long wire-bonds, which can reduce the output power.

The system shows the beam-forming capability after being tested with two different methods. The first method used was our frequency domain phase measurement technique using a power combiner instead of physical antennas. Although this technique exhibits an accuracy problem, especially for small phase steps, it showed that the proper phases are present at the outputs of the power amplifiers.

The second approach to measure the beam-forming used physical antennas and the radiation pattern of the two antennas beside each other was measured while rotating the transmitter with respect to the receiver antenna to measure the amplitude at different 
angles. This final measurement showed the beam-forming capability for six different phases accurately.

To summarize, the three main contributions of my research work, along with the design of the individual components, are:

- a complete phase noise analysis of a bipolar RTWO;

- an innovative, high frequency, low cost method for accurate phase shift measurements; and

- the monolithic integration of the multi-phase RTWO into a full phased-array system. ;

The following is a list of articles containing various aspects of the work presented in this thesis:

1 K. T. Ansari, T. N. Ross, P. M. Gamand, and C. Plett, "Frequency Domain Phase Shift Measurement Technique Applied to a Multiphase Rotary Travelling-Wave VCO”, IEEE Microwave and Wireless Components Letters.

2 R. Smith, K. Ansari, J. Rogers, and C. Plett, "Tuning of the Relative Phases of a Rotary Travelling-wave Oscillator", accepted (with some reviews) to Microwave and Wireless Components Letters.

3 K. T. Ansari, P. M. Gamand, and C. Plett, "Ku-Band Integrated Building Blocks for Phased-Array Transmitter Design in SiGe:C BiCMOS” IEEE Bipolar/BiCMOS Circuits and Technology Meeting, October 2015. 
4 K. T. Ansari, T. N. Ross and C. Plett, "Ku-band high output power multi-phase rotary travelling-wave $\mathrm{VCO}$ in SiGe BiCMOS", Proc. IEEE European Microwave Integrated Circuits Conference, pp. 97-100, October 2013.

5 K. T. Ansari, M. C. E. Yagoub, and C. Plett, "A Low-Power 0.4-22GHz CMOS Cascode Distributed Amplifier for Optical Communication Systems", Proc. IEEE Int. Conf. Electronics circuits and systems pp. 387-390, Hammamet, Tunisia, December 2009.

\subsection{Future Work}

Although the system presented in this thesis demonstrates the functionality of the beam-forming system based on an RTWO with relatively low power consumption, the silicon die is relatively large when compared to previously published works. This large area is mainly due to the geometry of the distribution network; the RTWO, switches and power amplifiers are compact in comparison.

It appears that a system-on-chip (SoC) implementation is the most cost-effective way to realize a phased array system, as the alternative is a system-in-package (SiP) where transitions from silicon to (perhaps) LTCC or to other carriers are required to distribute the phases - only to reach the switches and amplifiers which must then also be integrated into the package, and transitions are needed to connect to these.

As a result, future work could include utilizing slow-wave transmission lines to enable a more compact implementation of the distribution network and reduce the silicon area consumed. 
As well, future work could consist of a four-element array to achieve higher output power and a beam with higher directivity. To assist in performing less challenging, but less realistic, measurements a series of off-chip phase shifters could also be used to eliminate the need for antennas.

Another idea is to integrate a phase detector and control circuitry after the power amplifier, in order to measure the angle between the different elements and adjust the output phases of the RTWO automatically. A proof of concept has been demonstrated (without the distribution network and PAs) with another student, Raleigh Smith and the results of that work have been submitted to the IEEE Microwave and Wireless Components Letters. Incorporating this same idea after the PAs, however, would eliminate the need for a precise distribution network and would increase the accuracy of the entire system.

Finally, the oscillator could be integrated into a phased-locked loop to ensure that the frequency does not drift as much, and this would bring the project one step closer to being a practical product. 


\section{Bibliography}

[1] J. Wood, T. C. Edwards, and S. Lipa, "Rotary travelling-wave oscillator arrays: A new clock technology," IEEE Journal of Solid-State Circuits, vol. 36, no. 11, pp. 1654-1665, November 2001.

[2] Z. Bai, X. Zhou, R.D. Mason, and G. Allan, "Low-phase noise clock distribution network using rotary traveling-wave oscillators and built-in self-test phase tuning technique," IEEE Transactions on Circuits and Systems II: Express Briefs, vol. 62, no. 1, pp. 41-45, January 2015.

[3] B. Zhanjun, X. Zhou, and R. Mason, "A novel injection locked rotary traveling wave oscillator," in Proceedings of the IEEE International Symposium on Circuits and Systems, Melbourne, 2014, pp. 1768-1771.

[4] H. Krishnaswamy and $\mathrm{H}$ Hashemi, "A fully integrated 24GHz 4-channel phasedarray tranceiver in $0.13 \mu \mathrm{um}$ CMOS based on a variable-phase ring oscillator and PLL architecture," in Proceedings of the IEEE International Solid-State Circuits Conference, San Francisco, CA, 2007, pp. 124-126.

[5] K. T. Ansari, C. Plett, and P. Gamand, "Ku-band integrated building blocks for phased-array transmitter design in SiGe:C BiCMOS," in in Procedding of the IEEE Bipolar/BiCMOS Circuits and Technology Meeting, Boston, 2015. 
[6] D. Wei, R. Mahmoudi, and A. van Roermund, "A systematic design approach for phased-array receivers," in Proceedings of the IEEE Radio and Wireless Symposium, New Orleans, 2010, pp. 37-40.

[7] S. Ramant, N. S. Barker, and G. M. Rebeiz, "A W-band dielectric-lens-based integrated monopulse radar receiver," in Proceeding of the IEEE International Microwave Symposium Digest, Baltimore, MD, 1998, pp. 7-12.

[8] K. -J. Koh, J. W. May, and G. M. Rebeiz, "A milimeter-wave (40-45 GHz) 16element phased-array transmitter in 0.18-um SiGe BiCMOS technology," IEEE Journal of Solid-State Circuits, vol. 44, no. 5, pp. 1498-1509, May 2009.

[9] A. Hajimiri, H. Hashemi, A. Natarajan, X. Guan, and A. Komijani, "Integrated phased array systems in silicon," Proceedings of the IEEE, vol. 93, no. 9, pp. 16371655, September 2005.

[10] J. Rogers and C. Plett, Radio frequency integrated circuit design. Norwood, MA, United States of America: Artech House, 2003.

[11] B. P. Hopf, I. Wolff, and M. Guglielmi, "Coplanar MMIC active bandpass filters using negative resistance circuits," IEEE Transactions on Microwave Theory and Techniques, vol. 42, no. 12, pp. 2598-2602, August 2002.

[12] S. Bantab and Y. Koutsoyannopoulos, "CMOS active-LC bandpass filters with coupled-inductor Q-enhancement and center frequency tuning," IEEE Transactions 
on Circuits and Sytems II, vol. 51, no. 2, pp. 69-76, February 2004.

[13] Ha et al., "Gm-boosted complementary current-reuse colpitts VCO with low power and low phase noise," IEEE Microwave and Wireless Components Letters, vol. 24, no. 6, pp. 418-420, April 2014.

[14] W. Hui and A. Hajimiri, "A novel tuning technique for distributed voltage controlled oscillators," in Proceedings of the IEEE International Circuits and Systems, Geneva, 2000, pp. 57-60.

[15] V. Honkote and B. Taskin, "Capacitive load balancing for mobius implementation of standing wave oscillator," in Proceedings of the IEEE International Midwest Symposium on Circuits and Systems, Cancun, 2009, pp. 232-235.

[16] F. O'Mahony, C. P. Yue, M.A. Horowitz, and S. S. Wong, "Design of a $10 \mathrm{GHz}$ clock distribution network using coupled standing-wave oscillators," in Proceedings of the Design Automation Conference, 2003, pp. 682-687.

[17] J. -C. Chien and L. -H. Lu, "Design of wide-tuning-range millimeter-wave CMOS VCO with a standing-wave architecture," IEEE Journal of Solid-State Circuits, vol. 42, no. 9, pp. 1942-1952, September 2007.

[18] W. F. Andress and D. Ham, "Standing wave oscillators utilizing wave-adaptive tapered transmission lines," IEEE Journal of Solid-State Circuits, vol. 40, no. 3, pp. 638-651, March 2005. 
[19] K. Takinami, R. Walsworth, S. Osman, and S. Beccue, "Phase-Noise Analysis in Rotary Traveling-Wave Oscillators Using Simple Physical Model," IEEE Transactions on Microwave Theory and Techniques, vol. 58, no. 6, pp. 1465-1474, May 2010.

[20] D. Ham and W. Andress, "A circular standing wave oscillator," in Digest of Tehcnical Papers of the IEEE International Solid-State Circuits Conference, San Fransisco, 2004, pp. 380-533.

[21] T.-H. Huang and P.-L. You, "27-GHz low phase-noise CMOS standing-wave oscillator for millimeter wave applications," in Proceedings of the IEEE MTT-S International Microwave Symposium Digest, Atlanta, GA, 2008, pp. 367-370.

[22] K. T. Ansari, C. Plett, and M. C. E. Yagoub, "A low-power 0.4-22GHz CMOS cascode distributed amplifier for optical communication systems," in Proceedings of the IEEE International Conference on Electronics, Circuits, and Systems, Hammamet, 2009, pp. 387-390.

[23] H. Wu and A. Hajimiri, "Silicon-based distributed voltage-controlled oscillators," IEEE Journal of Solid-State Circuits, vol. 36, no. 3, pp. 493-502, March 2001.

[24] Z. Skvor, S. R. Saunders, and C. S. Aitchison, "Novel decade electronically tunable microwave oscillator based on the distributed amplifier," Electronics Letters, vol. 28, no. 17, pp. 1647-1648, August 2002. 
[25] D. Guckenberger and K. T. Kornegay, "Design of a differential distributed amplifier and oscillator using close-packed interleaved transmission lines," IEEE Journal of Solid-State Circuits, vol. 40, no. 10, pp. 1997-2007, October 2005.

[26] C. J. White and A. Hajimiri, "Phase noise in distributed oscillators," Electronics Letters, vol. 38, no. 23, pp. 1453-1454, November 2002.

[27] S. Kimura and Y. Imai, "0-40 GHz GaAs MESFET distributed baseband amplifier ICs for high-speed optional transmission," IEEE Transactions on Microwave Theory and Techniques, vol. 44, no. 11, pp. 2076-2082, August 2002.

[28] M. Kawashima, H. Hayashi, T. Nakagawa, and K. Araki, "A low-noise distributed amplifier using cascode-connected BJT terminal circuit," in Proceedings of the IEEE Asia-Pacific Microwave Conference, Taipei, 2001, pp. 21-24.

[29] J.-F. Chang and Y.-S. Lin, "3-10 GHz low-power, low-noise CMOS distributed amplifier using splitting-load inductive peaking and noise-suppression techniques," Electronics Letters, vol. 45, no. 20, pp. 1033-1035, September 2009.

[30] G. Le Grand de Mercey, "A 18GHz rotary traveling wave VCO in CMOS with I/Q outputs," in Proceedings of the European Solid-State Circuits Conference, Estoril, Portugal, 2003, pp. 489-492.

[31] F. Ben Abdeljelil et al., "Application of Bottom-Up Methodology to RTW VCO," in Proceedings of the IEEE Computer Society Annual Symposium on VLSI, 
Montpellier, France, 2008, pp. 46-50.

[32] D. -W. Kang and J. -G., Min, B. -W., Rebeiz, G. M. Kim, "Single and four-element Ka-band transmit/receive phased-array silicon RFICs with 5-bit amplitude and phase control," IEEE Transactions on Microwave Theory and Techniques, vol. 57, no. 12, pp. 3534-3543, December 2009.

[33] H. Zarei and D. J. Allstot, "A low-loss phase shifter in $180 \mathrm{~nm}$ CMOS for multipleantenna receivers," in Digest of Technical Papers in IEEE International Solid-State Circuits Conference, San Francisco, CA, 2004, pp. 392-534.

[34] H. Krishnaswamy and H. Hashemi, "A 4-channel 24-27 GHz UWB phased array transmitter in $0.13 \mu \mathrm{m}$ CMOS for vehicular radar," in Proceedings of the IEEE Custom Integrated Circuits Conference, San Jose, 2007, pp. 753-756.

[35] H. Krishnaswamy and H. Hashemi, "A fully integrated $24 \mathrm{GHz}$ 4-channel phasedarray transceiver in $0.13 \mathrm{um}$ CMOS based on a variable-phase ring oscillator and PLL architecture," in Digest of Technical Ppaers in IEEE International Solid-State Circuits Conference, San Fransisco, 2007, pp. 124-591.

[36] A. Natarajan, A. Komijani, and A. Hajimiri, "A fully integrated 24-GHz phasedarray transmitter in CMOS," IEEE Journal of Solid-State Circuits, vol. 40, no. 12, pp. 2502-2514, December 2005.

[37] K. T. Ansari, T. N. Ross, and C. Plett, "Ku-band high output power multiphase 
rotary travelling-wave VCO in SiGe BiCMOS," in Proceedings of the European Microwave Integrated Circuits Conference, Nuremberg, Germany, 2013, pp. 97100.

[38] H.-H. Hsieh, Y.-C. Hsu, and L- H. Lu, "A 15/30-GHz dual-band multiphase voltagecontrolled oscillator in 0.18- $\mu \mathrm{m}$ CMOS," IEEE Transactions on Microwave Theory and Techniques, vol. 55, no. 3, pp. 474-483, March 2007.

[39] C. Zhang, Z. Wang, Y. Zhao, and S. M. Park, "A 15 GHz, -182 dBc/Hz/mW FOM, Rotary Traveling Wave VCO in $90 \mathrm{~nm}$ CMOS," IEEE Microwave and Wireless Components Letters, vol. 22, no. 4, pp. 206-208, March 2012.

[40] A. Mirzaei, M. E. Heidari, R. Bagheri, S. Chehrazi, and A. A Abidi, "The quadrature LC oscillator: A complete portrait based on injection locking," IEEE Journal of Solid-State Circuits, vol. 42, no. 9, pp. 1916-1932, September 2007.

[41] D. Johns and K. Martin, Analog integrated circuit design. New York, United Stated of America: John Wiley \& Sons, Inc, 1997.

[42] H. Darabi and A. A. Abidi, "Noise in RF-CMOS mixers: a simple physical model," IEEE Journal of Solid-State Circuits, vol. 35, no. 1, pp. 15-25, January 2000.

[43] A. A. Abidi, "High-frequency noise measurement on FET's with small dimensions," IEEE Transactions on Electron Devices, vol. 33, no. 11, pp. 1801-1805, November 1986. 
[44] A. Tasic, W. A. Serdijn, and J. R. Long, "Spectral analysis of phase noise in bipolar LC-oscillator-theory, verification, and design," IEEE Transaction on Circuits and Systems I: Regular Papers, vol. 57, no. 4, pp. 737-751, April 2010.

[45] J.-C. Chien and L.-H. Lu, "A 32-GHz rotary traveling-wave voltage controlled oscillator in 0.18- $\mu \mathrm{m}$ CMOS," IEEE Microwave and Wireless Components Letters, vol. 17, no. 10, pp. 724-726, October 2007.

[46] D. M. Pozar, Microwave Engineering. Hoboken, NJ: Wiley, 2005.

[47] M. Li and R. E. Amaya, "Design of mm-W fully integrated CMOS standing-wave VCOs using low-loss CPW resonators," IEEE Transactions on Circuits and Systems II, vol. 59, no. 2, pp. 78-82, February 2012.

[48] P. Gamand and D. Bajon, "Slow wave propagation using interconnections for IC technologies," Electronics Letters, vol. 26, no. 18, pp. 1443-1445, August 2002.

[49] K. -J. Koh, J. W. May, and G. M. Rebeiz, "A millimeter-wave (40-45 GHz) 16element phased-array transmitter in $0.18-\mu \mathrm{m}$ SiGe BiCMOS technology," IEEE Journal of Solid-State Circuits, vol. 44, no. 5, pp. 1498-1509, May 2009.

[50] S. Zihir, O. D. Gurbuz, A. Karroy, S. Raman, and G. M. Rebeiz, "A 60 GHz 64element wafer-scale phased-array with full-reticle design," in Proceedings of the IEEE International Microwave Symposium, Phoenix, 2015, pp. 17-22. 
[51] M.-C. Yeh et al., "Design and analysis of a miniature CMOS SPDT switch using body-floating technique to improve power performance," IEEE Transactions on Microwave Theory and Techniques, vol. 54, no. 1, pp. 31-39, January 2006.

[52] Q. Li and Y. P. Zhang, "CMOS T/R switch design: towards ultra-wideband and higher frequency," IEEE Journal of Solid-State Circuits, vol. 42, no. 3, pp. 563-570, March 2007.

[53] W. D. van Noort et al., "BiCMOS technology improvements for microwave application," in Proceedings of the IEEE Bipolar/BiCMOS Circuits and Technology Meeting, Monterey, CA, 2008, pp. 93-96.

[54] M. D. Yore, C. A. Nevers, and P. Cortese, "High-isolation low-loss SP7T pHEMT switch suitable for antenna switch modules," in Proceedings of the IEEE European Microwave Integrated Circuits Conference, Paris, 2010, pp. 69-72.

[55] H. Hashemi, X. Guan, A. Komijani, and A. Hajimiri, "A 24-GHz SiGe phased-array receiver- LO phase shifting approach," IEEE Transaction on Microwave Theory and Techniques, vol. 53, no. 2, pp. 614-626, February 2005.

[56] A. Komijani, A. Natarajan, and A. Hajimiri, "A 24-GHz, +14.5-dBm fully integrated power amplifier in 0.18- $\mu \mathrm{m}$ CMOS," IEEE Journal of Solid-State Circuits, vol. 40, no. 9, pp. 1901-1908, September 2005.

[57] F. Brecht and P. Reynaert, "Highly linear fully integrated wideband RF PA for LTE- 
advanced in 180-nm SOI," IEEE Transactions on Microwave Theory and Technique, vol. 63, no. 2, pp. 649-658, February 2015.

[58] J. Staudinger, "An overview of efficiency enhancements with application to linear handset power amplifiers," in Proceedings of the IEEE Radio Frequency Integrated Circuits (RFIC) Symposium, Seattle, WA, 2002, pp. 45-48.

[59] A. Komijani and A. Hajimiri, "A wideband $77 \mathrm{GHz}, 17.5 \mathrm{dBm}$ power amplifier in silicon," in Proceeding of the IEEE Custom Integrated Circuits Conference, 2005, pp. 571-574.

[60] J. William and R. Nakkeeran, "A compact CPW-fed UWB slot antenna with cross tuning stub," Pondicherry Engineering Collage, Puducherry, 2010.

[61] W. Menzel and W. Grabherr, "A microstrip patch antenna with coplanar feed line," IEEE Microwave and Guided Wave Letters, vol. 1, no. 11, pp. 340-342, November 1991.

[62] T. A. Milligan, Modern antenna design. New Jersey, United States of America: John Willey \& Sons, 2005.

[63] O. Katircioglu, H. Isel, O. Ceylan, F. Taraktas, and H. B. Yagci, "Comparing ray tracing, free space path loss and logarithmic distance path loss models in success of indoor localization with RSSI," in Telecommunications Forum, Belgrade, 2011, pp. 313-316. 
[64] W. A. Shittu, B. G. Bajoga, F. Anwar, and M. J. E. Salami, "Prediction of received signal power and propagation path loss in open/rural environments using modified Free-Space loss and Hata models," in Proceedinbgs of the IEEE International RF and Microwave Conference, Kuala Lumpur, 2008, pp. 126-130.

[65] N. Nomura, Y. Aoyagi, and Y. Sekine, "1 GHz high frequency Colpitts oscillator," in Proceedings of the IEEE International Frequency Control Symposium and Exposition, 2005, pp. 526-529.

[66] B. Koc, A. Koukab, and G. Dundar, "Phase noise in bipolar and CMOS VCO's - an analytical comparison," in Proceedings of the IEEE International Symposium on Circuits and Systems, Island of Kos, 2006.

[67] Y. Teng and B. Taskin, "Process variation sensitivity of the rotary traveling wave oscillator," in Proceedings of the International Symposium on Quality Electronic Design, Santa Clara, CA, 2011, pp. 236-242.

[68] V. Honkote and R. D. Nagarajan, "Process variation sensitivities of rotary traveling wave and mobius standing wave oscillators," in Proceedings of the International Symposium on Electronic System Design, Singapore, 2013, pp. 6-10.

[69] J. Phillips and K. Kundert, "Noise in mixers, oscillators, samplers, and logic an introduction to cyclostationary noise," in Proceedings of the IEEE Custom Integrated Circuits Conference, Orlandi, FL, 2000, pp. 431-438. 
[70] J. J, Rael and A. A. Abidi, "Physical processes of phase noise in differential LC oscillators," in Proceeding of the IEEE Custom Integrated Circuits Conference, 2000, pp. 569-572.

[71] D. Wang and X. Wang, "The performance comparison of CMOS vs bipolar VCO in SiGe BiCMOS technology," in Proceedings of the IEEE Radio Frequency Integrated Circuits (RFIC) Symposium, 2003, pp. 615-618.

[72] H. Krishnaswamy and $\mathrm{H}$ Hashemi, "A fully integrated $24 \mathrm{GHz}$ 4-channel ohasedarray tranceiver in $0.13 \mathrm{um} \mathrm{CMOS}$ based on a variable-phase ring oscillator and PLL architecture," in IEEE International Solid-State Circuits Conference, San Francisco, CA, 2007, pp. 124-126.

[73] S. Ramant, N. S. Barker, and G. M. Rebeiz, "A W-band dielectric-lens-based integrated monopulse radar receiver," in IEEE International Microwave Symposium Digest, Baltimore, MD, 1998, pp. 7-12.

[74] K. -J. Koh, J. W. May, and G. M. Rebeiz, "A millimeter-wave (40-45 GHz) 16element phased-array transmitter in 0.18-um SiGe BiCMOS technology," IEEE Journal of Solid-State Circuits, vol. 44, no. 5, pp. 1498-1509, May 2009.

[75] W. D. van Noort et al., "BiCMOS technology improvements for microwave application," in IEEE Bipolar/BiCMOS Circuits and Technology Meeting, Monterey, CA, 2008, pp. 93-96. 
[76] J. Staudinger, "An overview of efficiency enhancements with application to linear handset power amplifiers," in IEEE Radio Frequency Integrated Circuits (RFIC) Symposium, Seattle, WA, 2002, pp. 45-48.

[77] D. Wang and X. Wang, "The performance comparison of CMOS vs bipolar VCO in SiGe BiCMOS technology," in IEEE Radio Frequency Integrated Circuits Symposium, 2003, pp. 615-618.

[78] J. J, Rael and A. A. Abidi, "Physical processes of phase noise in differential LC oscillators," in Proceeding of IEEE Custom Integrated Circuits Conference, 2000, pp. 569-572. 